\title{
EFFICACY OF COMMON DISINFECTANT/CLEANING AGENTS IN INACTIVATING MURINE NOROVIRUS AND FELINE CALICIVIRUS AS SURROGATE VIRUSES FOR HUMAN NOROVIRUS
}

\author{
by \\ Stephanie Chiu \\ B.Sc., The University of British Columbia, 2006 \\ A THESIS SUBMITTED IN PARTIAL FULFILLMENT OF \\ THE REQUIREMENTS FOR THE DEGREE OF \\ MASTER OF SCIENCE \\ in
}

The Faculty of Graduate Studies

(Food Science)

THE UNIVERSITY OF BRITISH COLUMBIA

(Vancouver)

February 2013

(C) Stephanie Chiu, 2013 


\begin{abstract}
Background/Objectives: Norovirus, a major cause of foodborne gastrointestinal infection, cannot be propagated in cell culture. Limited information exists on the effectiveness of disinfectants and cleaning agents. The objective of this study was to determine the efficacy of common types of disinfectants/cleaning agents used in health care facilities in British Columbia on surrogate viruses, murine norovirus (MNV-1) and feline calicivirus (FCV). Sodium hypochlorite, accelerated hydrogen peroxide (AHP) and a quaternary ammonium compound (QUAT) were assessed.
\end{abstract}

Methods: A virus suspension of known concentration (with or without a soil load) was deposited onto stainless steel discs under wet or dry load conditions and exposed to defined concentrations of the disinfectant/cleaning agent for 1,5 or 10 minute contact time using the quantitative carrier test (QCT-2) method. Virus inactivation was determined by plaque assay.

Results: Sodium hypochlorite at 1350 ppm inactivated MNV-1 after 5 minutes with a $\sim 5.5$ to 6.5 $\log _{10}$ reduction, whereas it took twice as long to inactivate the FCV with $\sim 4.6$ to $5.6 \log _{10}$ reduction. After 5 minutes, 2700 ppm of sodium hypochlorite was able to inactivate MNV-1 and FCV. Accel at 35000 ppm AHP inactivated MNV-1 after 10 minutes with a $~ 5.6$ to $6.5 \log _{10}$ reduction, whereas at $3500 \mathrm{ppm}, \mathrm{FCV}$ was inactivated by a $\sim 5 \log _{10}$ reduction. CaviCide at 2800 ppm QUAT and Virox 5 at 5000 ppm AHP were unable to inactivate MNV-1. $\mathrm{T}^{3} 6$ at 2000 ppm QUAT and $70 \%$ ethanol was effective in inactivating MNV-1 with a $>6 \log _{10}$ reduction after 5 minutes, but only resulted in a $<3 \log _{10}$ reduction of FCV after 10 minutes. 
Conclusions: The results have demonstrated that sodium hypochlorite at $1350 \mathrm{ppm}$ after 10 minutes or $2700 \mathrm{ppm}$ at shorter contact times of 5 minutes was more effective in reducing the viral load of both MNV-1 and FCV on stainless steel surfaces than ready-to-use AHP and QUAT products. Concentrated AHP products were only effective against MNV-1 when used at a concentration of $35000 \mathrm{ppm}$ for 10 minutes. QUATs without ethanol were ineffective against both surrogate viruses and are therefore not indicated for disinfecting environmental surfaces contaminated with norovirus. 


\section{Preface}

The University of British Columbia (UBC) Department of Health, Safety and Environment

Laboratory Biological Safety Course was completed on October 10, 2006, prior to the start of the project.

The UBC Biosafety Committee had approved of this project, Certificate Number B10-0140. 


\section{Table of Contents}

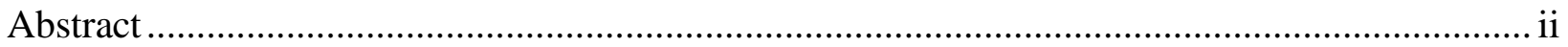

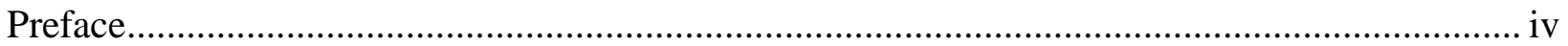

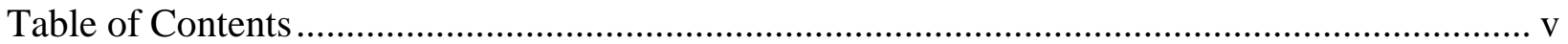

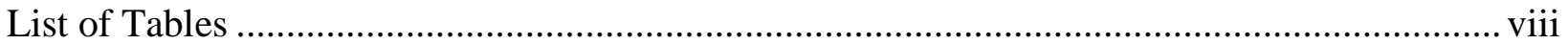

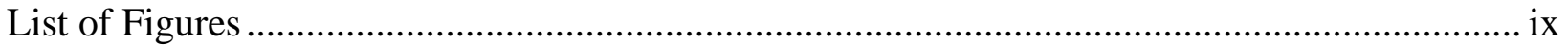

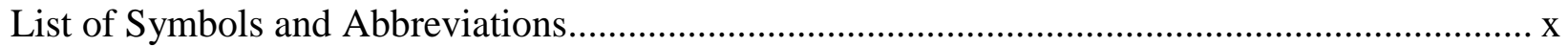

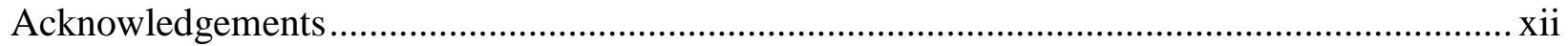

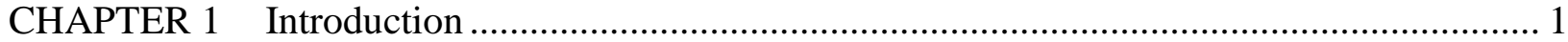

$1.1 \quad$ Burden of Disease Associated with Norovirus .............................................................. 1

1.2 Background Information and Literature Review …………........................................ 3

1.2.1 Virology of Noroviruses ............................................................................... 3

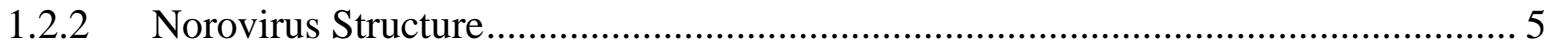

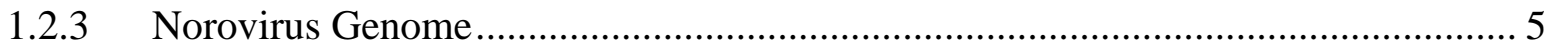

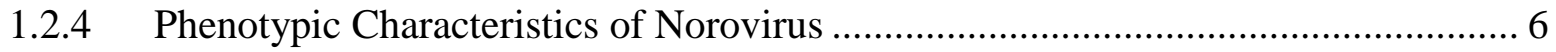

1.2.5 Norovirus Detection Methods ..................................................................... 10

1.2.6 Clinical Presentations of Norovirus Infections .................................................... 13

1.2.7 Epidemiology and Transmission of Norovirus ................................................... 15

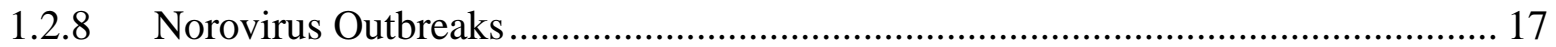

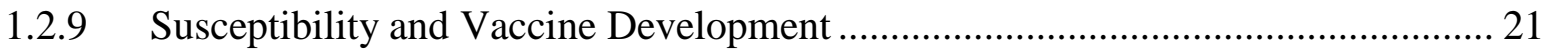

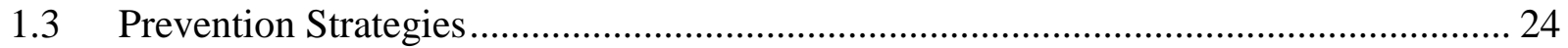

1.3.1 Infection Control and Outbreak Prevention ....................................................... 24

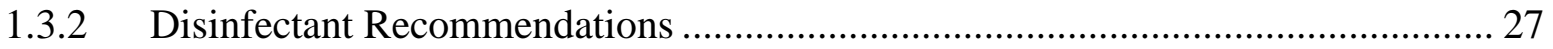

1.4 Surrogate Viruses of Human Norovirus: MNV-1 and FCV ………………................ 29

1.4.1 Murine Norovirus (MNV-1) …………….................................................. 30

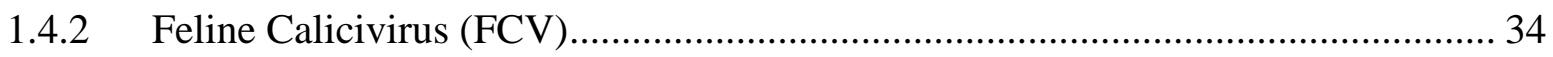

1.5 Disinfectants and Cleaning Agents ......................................................................... 40

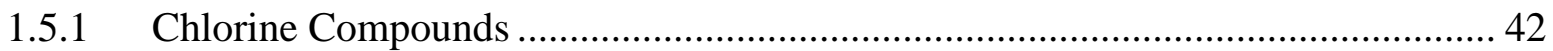

1.5.2 Quaternary Ammonium Compounds ............................................................... 43

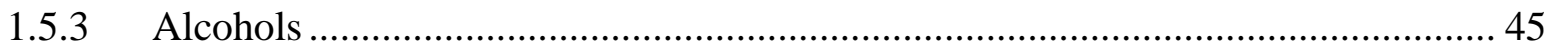

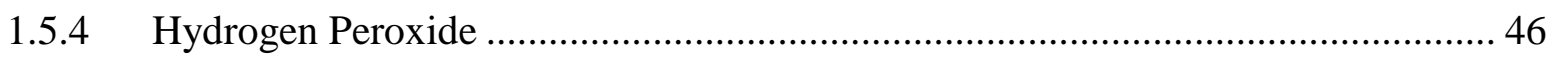

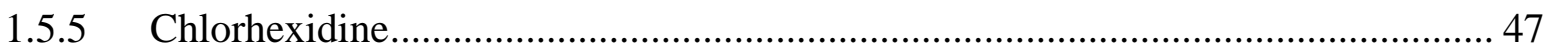

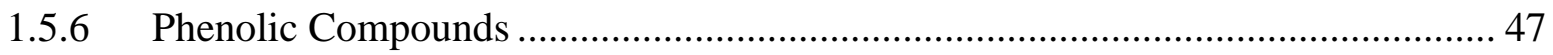

1.6 Disinfectant Studies on Surrogate Viruses: MNV-1 and FCV ……………................ 48 


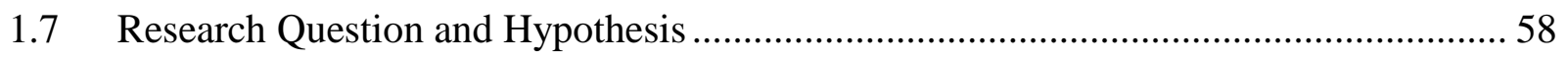

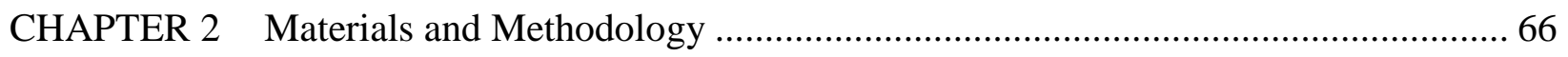

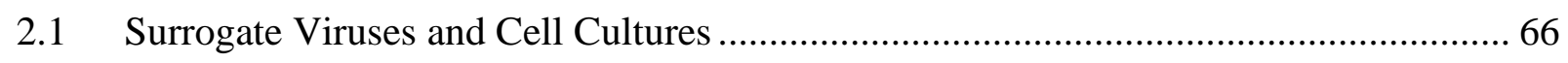

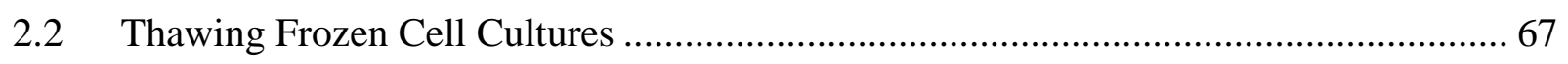

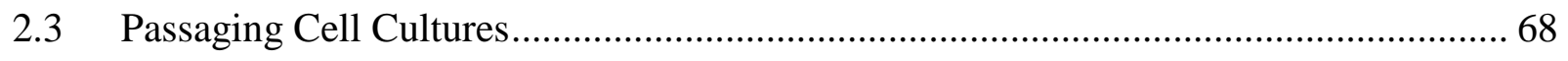

2.4 Harvesting and Preservation of Cells for Frozen Stock …….......................................... 69

2.5 Virus Propagation for Frozen Stock .......................................................................... 70

2.6 Virus Inactivation Tests: Quantitative Carrier Test 2 (QCT-2) Method ......................... 71

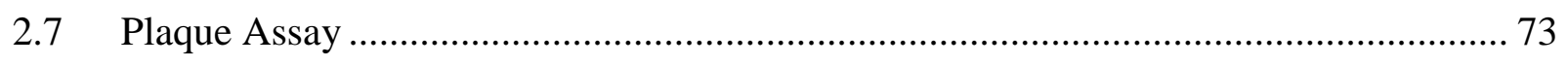

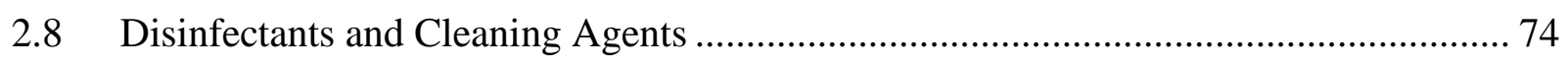

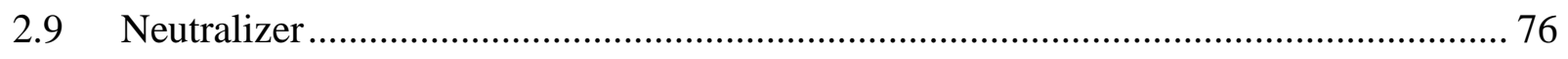

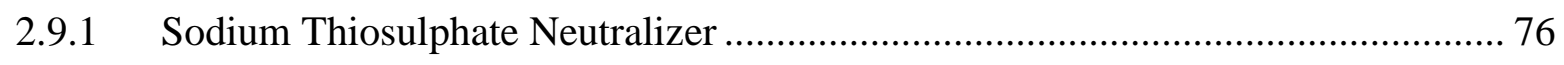

2.9.2 Letheen Broth Neutralizer................................................................................ 77

2.10 Additional Control Tests for Virucidal Activity.................................................... 77

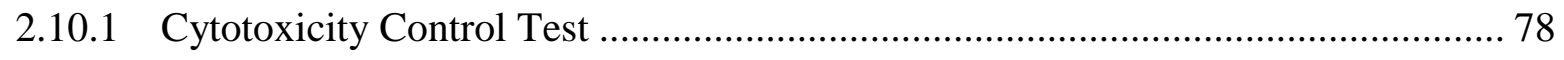

2.10.2 Interference with Virus Infectivity Control Test ................................................... 79

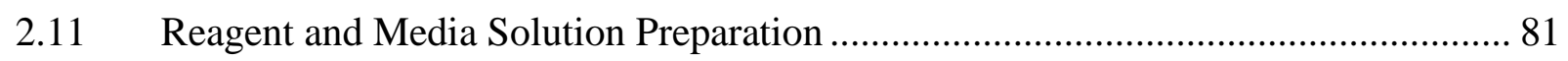

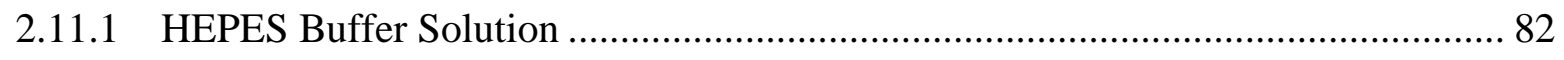

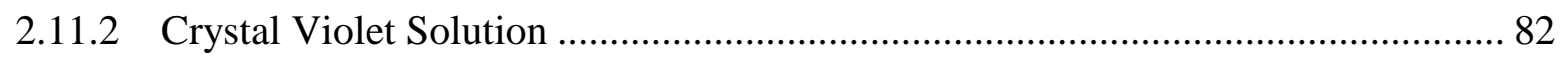

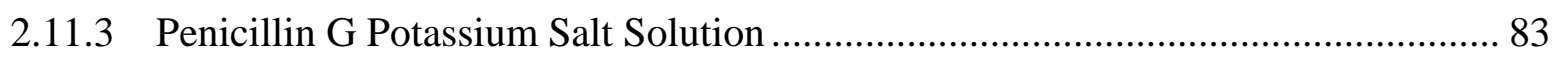

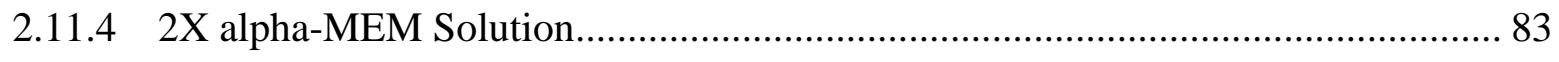

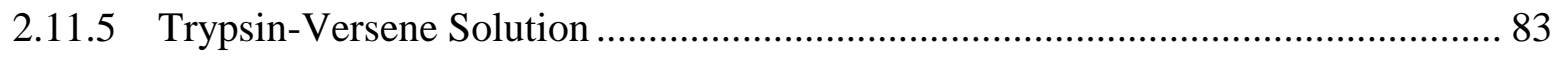

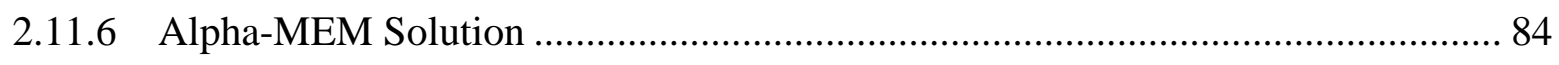

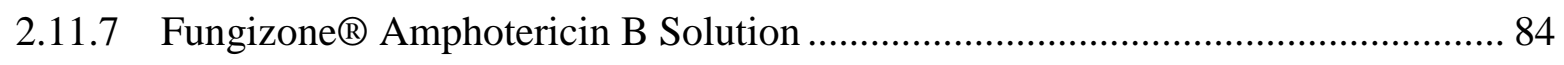

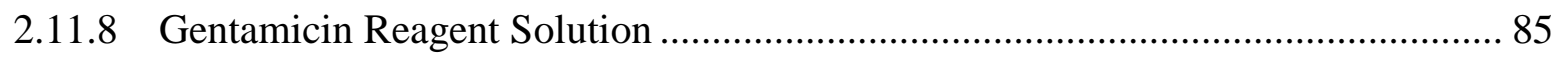

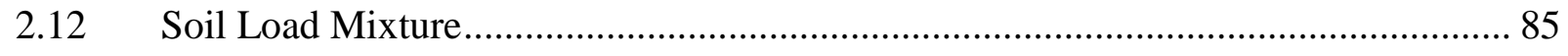

2.12 .1 Tryptone

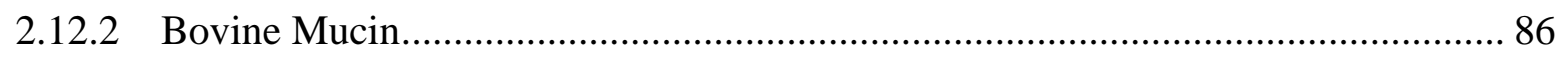

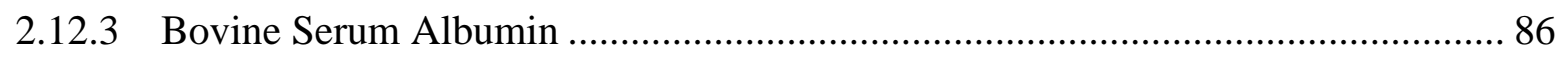

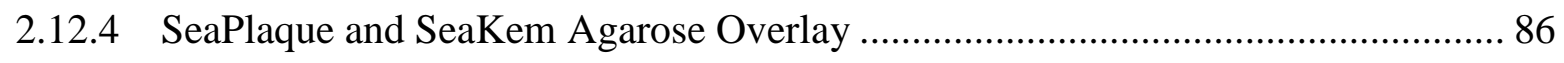

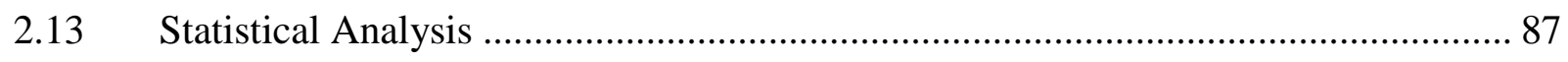

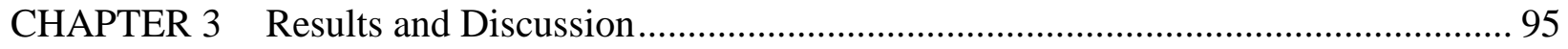

3.1 Efficacy of Sodium Hypochlorite (Domestic Miraclean Bleach) against MNV-1 and FCV, with and without Soil Load, under Wet and Dry Load Conditions..................... 100 
3.2 Efficacy of Accelerated Hydrogen Peroxide (Accel and Virox 5) against MNV-1 and FCV, with and without Soil Load, under Wet and Dry Load Conditions. 102

3.3 Efficacy of QUAT (CaviCide) and QUAT-Alcohol $\left(\mathrm{T}^{3} 6\right)$ against MNV-1 and FCV, with and without Soil Load, under Wet and Dry Load Conditions.............................. 106

3.4 Effect of Drying on Viral Titer ......................................................................... 107

3.5 Cytotoxicity and Virus Infectivity Control Test ..................................................... 113

3.5.1 Cytotoxicity Observations for RAW 264.7 and CRFK Cells ............................... 114

3.5.2 Virus Infectivity of MNV-1 towards RAW 264.7 Cells..................................... 116

3.5.3 Virus Infectivity of FCV towards CRFK Cells................................................. 118

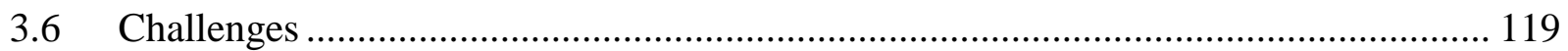

3.7 Mechanism of Virucidal Activity and Overall Assessment of Most Suitable Surrogate Virus for Human Norovirus .................................................................................. 123

CHAPTER 4 Conclusion and Recommendations for Future Work ........................................ 140

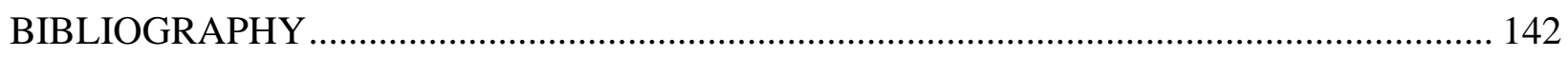

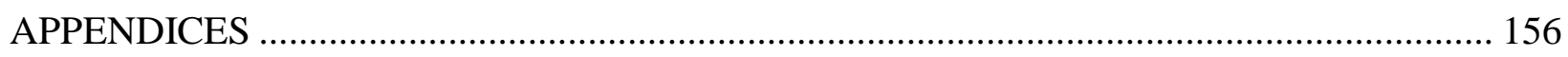

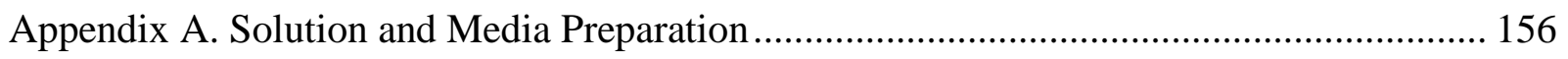

Appendix B. Statistical Analysis of Virus Infectivity Control Tests and Viral Drying ......... 158

Appendix C. Photo Images of Experimental Design and Virus Plaques ................................ 172

Appendix D. Conversion of Revolutions Per Minute (rpm) to Centrifugal Force $(\times g)$........ 177 


\section{List of Tables}

Table 1. Comparison of temperature, environmental and $\mathrm{pH}$ stability of norovirus, hepatitis A virus, Staphylococcus aureus and Escherichia coli O157:H7.

Table 2. Primers and probes used in real-time quantitative RT-PCR for detecting norovirus genogroups I and II.

Table 3. Summary of effectiveness of commonly used disinfectants, $\mathrm{pH}$ and temperature on MNV-1 and FCV surrogates of human norovirus.

Table 4. List of disinfectants tested, manufacturers, active ingredients, concentrations tested and classification.

Table 5. Manufacturer recommendations for the five disinfectants used in the study.

Table 6. Cytotoxicity control test of RAW 264.7 macrophage mouse and CRFK cells from exposure to selected disinfectants and reagents.

Table 7. Interference with virus infectivity control test of MNV-1 and FCV to infect host cells RAW 264.7 macrophage mouse and CRFK cells from exposure to selected disinfectants and reagents.

Table 8. Mean log and percentage differences in viral titer after drying for MNV-1 and FCV controls

Table 9. Summary of the most effective concentrations and contact times of commonly used disinfectants against MNV-1 and FCV, surrogates of human norovirus, with and without soil load.

Table 10. Virucidal efficacy of commonly used disinfectants against MNV-1, a surrogate of human norovirus, under wet and dry load conditions, with and without soil load, at 1, 5 and 10 minute contact times.

Table 11. Virucidal efficacy of commonly used disinfectants against FCV, a surrogate of human norovirus, under wet and dry load conditions, with and without soil load, at 1, 5 and 10 minute contact times. 


\section{List of Figures}

Figure 1. Diagram of Calicivirus family, genus, genogroup and genotype................................ 64

Figure 2. Norovirus genome with protein products and positions of regions A-D used for detection and genotyping. 65

Figure 3. Experimental design flowchart of the study including surrogate viruses MNV-1 and FCV, soil load versus no soil load, wet versus dry load conditions, and contact time................. 91

Figure 4. QCT-2 method for virus inactivation and plaque assay method. .................................. 92

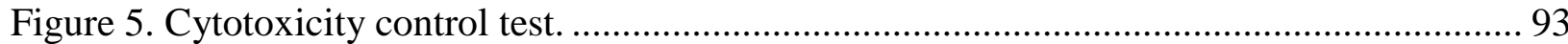

Figure 6. Interference with virus infectivity control test. .......................................................... 94

Figure 7. Effectiveness of sodium hypochlorite against MNV-1, (A) without soil load and (B)

with soil load, at 1,5 and 10 minute contact times, under wet and dry load conditions............ 128

Figure 8. Effectiveness of sodium hypochlorite against FCV, (A) without soil load and (B) with

soil load, at 1, 5 and 10 minute contact times, under wet and dry load conditions. ................... 129

Figure 9. Effectiveness of Accel (AHP) against MNV-1, (A) without soil load and (B) with soil

load, at 1,5 and 10 minute contact times, under wet and dry load conditions.

Figure 10. Effectiveness of Accel (AHP) against FCV, (A) without soil load and (B) with soil

load, at 1, 5 and 10 minute contact times, under wet and dry load conditions.......................... 131

Figure 11. Effectiveness of CaviCide (QUAT), Virox 5 (AHP) and $\mathrm{T}^{3} 6$ (QUAT-alcohol) against

MNV-1 (without soil load) at 1, 5 and 10 minute contact times, under wet and dry load

conditions.

Figure 12. Effectiveness of Virox 5 (AHP) against MNV-1 (with soil load) at 1, 5 and 10 minute

contact times, under wet and dry load conditions.............................................................. 132

Figure 13. Effectiveness of CaviCide (QUAT), Virox 5 (AHP) and T ${ }^{3} 6$ (QUAT-alcohol) against FCV (without soil load) at 1, 5 and 10 minute contact times, under wet and dry load conditions.

Figure 14. Effectiveness of Virox 5 (AHP) against FCV (with soil load) at 1, 5 and 10 minute contact times, under wet and dry load conditions. 


\section{List of Symbols and Abbreviations}

\begin{tabular}{|c|c|}
\hline $3 \mathrm{C}$ & 3C-Like Protease \\
\hline AHP & Accelerated Hydrogen Peroxide \\
\hline Alpha-MEM & Alpha-Minimum Essential Medium \\
\hline ANOVA & Analysis of Variance \\
\hline ATCC & American Type Culture Collection \\
\hline BCPHMRL & $\begin{array}{l}\text { British Columbia Public Health \& Microbiology Reference } \\
\text { Laboratories }\end{array}$ \\
\hline $\mathrm{BCCDC}$ & British Columbia Centre for Disease Control \\
\hline BGA & Blood Group Antigen \\
\hline BSA & Bovine Serum Albumin \\
\hline $\mathrm{CDC}$ & Centers for Disease Control and Prevention \\
\hline CRFK & Crandell Reese Feline Kidney \\
\hline CTE & Cytotoxic Effects \\
\hline DMSO & Dimethyl sulfoxide \\
\hline D-Value & Decimal Reduction Time \\
\hline EBSS & Earle's Balanced Salt Solution \\
\hline EDTA & Ethylenediaminetetraacetic acid disodium salt \\
\hline $\mathrm{EHO}$ & Environmental Health Officer \\
\hline ELISA & Enzyme Linked Immunosorbent Assay \\
\hline EPA & Environmental Protection Agency \\
\hline FBS & Fetal Bovine Serum \\
\hline FBVE & Foodborne Viruses in Europe \\
\hline FCV & Feline Calicivirus \\
\hline GEP & Genome Equivalent Particles \\
\hline HAV & Hepatitis A Virus \\
\hline HBGA & Histo-Blood Group Antigen \\
\hline HIV & Human Immunodeficiency Virus \\
\hline HSD & Honestly Significant Difference \\
\hline $\mathrm{ID}_{50}$ & Infective Dose \\
\hline MNV-1 & Murine Norovirus \\
\hline NTP & Nucleoside Triphosphatase \\
\hline ORF & Open Reading Frame \\
\hline PBS & Phosphate-buffered saline \\
\hline PFU & Plaque Forming Unit \\
\hline PPE & Personal Protective Equipment \\
\hline QCT-2 & Quantitative Carrier Test-2 \\
\hline QUATs & Quaternary Ammonium Compounds \\
\hline RdRp & RNA-Directed RNA Polymerase \\
\hline RHDV & Rabbit Haemorrhagic Disease Virus \\
\hline RPM & Revolutions per minute \\
\hline RT-PCR & Reverse Transcription-Polymerase Chain Reaction \\
\hline RT-qPCR & Reverse Transcription-Quantitative Polymerase Chain Reaction \\
\hline RTU & Ready-to-use \\
\hline $\mathrm{TCID}_{50}$ & Tissue Culture Infectious Dose \\
\hline
\end{tabular}


TV

USEPA

VLP

VP1

VP2

VPG

VS-FCV
Tulane Virus

United States Environmental Protection Agency

Virus-Like Particle

Viral Protein 1

Viral Protein 2

Viral Genome-Linked Protein

Virulent Systemic Feline Calicivirus 


\section{Acknowledgements}

I would like to thank the Community and Hospital Infection Control Association of Canada (CHICA-Canada) for awarding a research grant to help fund this project. I would also like to thank CHICA-Canada for supporting this research and Dr. Brent Skura for graciously providing financial assistance. I would like to thank my committee members, Dr. Judith Isaac-Renton, Dr. Martin Petric, Lorraine McIntyre and Bruce Gamage, my supervisor, Dr. Brent Skura, for their continued support and advice, and the support staff of the British Columbia Public Health \& Microbiology Reference Laboratories (BCPHMRL).

My laboratory experiments were conducted in the BCPHMRL located at the British Columbia Centre for Disease Control (BCCDC). The laboratory technologists from the BCPHMRL Virology Program were very helpful, thoughtful, encouraging and knowledgeable. I would like to send a heartfelt thanks to all BCPHMRL staff that helped me along the way, specifically Joe Fung, Julie Wong, Brian Auk, Ron Gillies, Louella D’Silva, Raymond Ma, Benny Hoy, Tracy Chan, Julie Ho, Annie Mak and Alan McNabb. I would like to especially thank David Lawrence for training me on growing viruses and performing plaque assays. The many laboratory skills I learned from David were invaluable.

Lastly, I would like to thank my family for their financial support and understanding, and my friends for their words of encouragement throughout the course of my M.Sc. program. 


\section{CHAPTER 1 Introduction}

\subsection{Burden of Disease Associated with Norovirus}

Acute gastroenteritis, the inflammation of the stomach and small intestine, is characterized by nausea, vomiting and diarrhea. It may also be accompanied by other symptoms such as fever and chills. Acute gastroenteritis can be caused by viral, bacterial or parasitic infection, or can result from intoxication by bacterial toxins (Nuermberger, 2005). Norovirus has emerged as the major cause of foodborne gastrointestinal infections based on the recent development and use of sensitive nucleic acid based diagnostic techniques (Nuermberger, 2005; Patel et al., 2009).

Through active and passive surveillance, it is estimated that each year 31 major pathogens in the United States cause 9.4 million episodes of foodborne illness, of which $58 \%$ were caused by norovirus, $11 \%$ by Salmonella enterica, $10 \%$ by Clostridium perfringens, $9 \%$ by Campylobacter spp., and $3 \%$ by Staphylococcus aureus (Centers for Disease Control and Prevention, 2011a; Scallan et al., 2011). Norovirus is highly contagious, and can spread quickly in premises such as hospitals, daycare centres, residential and domestic homes (Barker et al., 2004).

Of all infectious intestinal diseases, noroviruses accounted for an estimated $6 \%$ in England and $11 \%$ in the Netherlands (Fretz et al., 2005). The Centers for Disease Control and Prevention (CDC) in the United States estimates that each year, more than 21 million cases of acute gastroenteritis are caused by norovirus infection (Centers for Disease Control and Prevention, 2011c). Noroviruses were shown to be responsible for over $85 \%$ of all nonbacterial outbreaks of gastroenteritis reported from 1995 to 2000 based on information from 10 data surveillance systems in the Foodborne Viruses in Europe (FBVE) Network (Lopman et al., 2003). In 2006, 
the number of norovirus outbreaks from 13 European countries was reported at $4074(11.2$ outbreak rate per million population size) (Kroneman et al., 2007). In the United States, $39 \%$ of all foodborne outbreaks were reported to be due to norovirus infection (Centers for Disease Control and Prevention, 2010). Between 300 and 400 norovirus outbreaks are reported to the National Enteric Surveillance Program each year in Canada (Public Health Agency of Canada, 2005).

In the late 1990s, sensitive and simpler assays such as reverse transcription-polymerase chain reaction (RT-PCR) were adopted at the CDC for detection of norovirus in outbreaks. Accordingly, norovirus was identified as the etiologic agent in $93 \%$ of outbreaks of nonbacterial gastroenteritis in tests submitted to the CDC from 1997 to 2000 . An analysis of 8271 foodborne outbreaks reported to the CDC from 1991 to 2000 showed that the introduction of RT-PCR greatly improved identification of the etiological source of outbreaks in the United States, up from less than $1 \%$ in 1991 to $12 \%$ in 2000 (Widdowson et al., 2005). Harris et al. (2008) used regression analysis to determine the number of deaths in England and Wales from 2001-2006, and based on the model, the results suggested that $20 \%$ of deaths for people 65 years of age or higher could be attributed to infectious intestinal diseases that were associated with norovirus infection. 


\subsection{Background Information and Literature Review}

\subsubsection{Virology of Noroviruses}

Noroviruses, previously called Norwalk virus or Norwalk-like viruses, were discovered and identified using immune electron microscopy to examine stool filtrate samples from a volunteer infected with a bacteria-free stool filtrate (the sample was obtained during a school outbreak in Norwalk, Ohio in 1968) (Kapikian et al., 1972). This became the prototype of the norovirus genus.

Noroviruses are single-stranded, positive-sense RNA viruses that belong to the Caliciviridae family (Lopman et al., 2004; Zingg et al., 2005). They are small, round, non-enveloped, icosahedral structured viruses that can range from 35 to $40 \mathrm{~nm}$ in diameter (Donaldson et al., 2010; Lopman et al., 2004; Zingg et al., 2005). The Caliciviridae family (Figure 1) includes the following genera: Norovirus, Sapovirus, Lagovirus, Vesivirus and proposed (but not official) genera, Nebovirus and Recovirus, which are pending (Green et al., 2000; Karst, 2010; Patel et al., 2009).

The Norovirus genus is composed of more than 40 virus genotypes (strains) that are divided into five genogroups based on sequence diversity in the complete capsid protein, VP1 (Donaldson et al., 2010; Patel et al., 2009). Most human noroviruses relevant to human disease are divided into genogroups I and II, based on the genetic diversity of the capsid or polymerase genes, and with a subset also clustered within genogroup IV (Goodridge et al., 2004; Patel et al., 2009). Data from early antigenic analyses and more recently extensive sequence analyses show noroviruses to be highly genetically variable (Radford et al., 2004). Genogroup II viruses have less genetic 
diversity than genogroup I (Hutson et al., 2004; Radford et al., 2004). Genogroup II includes both human and swine noroviruses (Patel et al., 2009). Genogroup II, genotype 4 noroviruses (GII.4) are the most common strains currently detected and are responsible for $70-80 \%$ of human norovirus outbreaks in the world (Donaldson et al., 2010). Out of 773 norovirus-related outbreaks reported to the CDC between 1994 and 2006, $81.4 \%$ were caused by genogroup II viruses and $44.2 \%$ were caused by GII.4 strains (Zheng et al., 2010). Genogroup IV viruses are rare in humans and their significance is currently unknown although they include both human and canine noroviruses. Genogroup III noroviruses are bovine noroviruses, whereas genogroup V are murine noroviruses (Patel et al., 2009; Radford et al., 2004).

There is $69-97 \%$ nucleotide identity in strains within a genotype, whereas strains of different genogroups have 51-56\% nucleotide similarity. There is substantial variability in structural proteins from strains within one genotype reaching up to $40 \%$ in the capsid amino acid sequence, whereas the strains of different genogroups can differ by more than $50 \%$ (Donaldson et al., 2010).

There is some evidence to suggest that evolution in some genotypes is sufficient enough to generate mutant clusters with new ligand-binding characteristics and antigenic properties. It has been shown in the GII.4 genotype that evolution can result in differential receptor binding and novel antigenic features (Donaldson et al., 2010). 


\subsubsection{Norovirus Structure}

The norovirus genome (Figure 2) is approximately $7.7 \mathrm{~kb}$ in size and contains three open reading frames (ORF) that encode structural and non-structural proteins (Donaldson et al., 2010; Hardy, 2005). The first x-ray crystallography of the norovirus capsid was performed by Prasad et al. (1999). The capsid consists of 180 copies of a single protein, with the capsid protein having a protruding $\mathrm{P}$ domain connected by a flexible hinge to a shell $\mathrm{S}$ domain which has a classical eight-stranded $\beta$-sandwich motif. The $\mathrm{S}$ domain is involved in the formation of the icosahedral shell, whereas the $\mathrm{P}$ domain forms the protrusion emanating from the shell. Three-dimensional structures of recombinant norovirus were studied using electron cryomicroscopy and computer image processing techniques at $\sim 22 \AA ̊$ resolution. Caliciviruses have a $\mathrm{T}=3$ icosahedral symmetry with 180 molecules of the capsid protein, which contain a protein shell between 100 and 145 A radius, organized into 90 dimers. The protein shell of the capsid protein exhibits protrusions at all local and strict two-fold axes that extend to an outer radius of $\sim 190 \AA$ and leaves large depressions at the icosahedral five and three-fold axes (Prasad et al., 1999).

\subsubsection{Norovirus Genome}

The 5' end of the genome is covalently coupled with protein VPg and the 3' end is polyadenylated (Hardy, 2005). The capsid of norovirus is composed of 180 copies of viral protein 1 (VP1), which is the major important component. Viral protein 2 (VP2), a minor structural protein, is also present in the capsid in low copy number. VP1 is divided into two domains: shell domain and protruding domain, which is further divided into two sub-domains, P1 and P2 (Donaldson et al., 2010). 
The first ORF (ORF1) of about $5 \mathrm{~kb}$ is located in the first two-thirds of the genome. This ORF encodes a $\sim 200 \mathrm{kDa}$ polyprotein that is auto-processed by a virally encoded 3C-like protease (3C) and yields non-structural replicase proteins that are essential for viral replications. The polyprotein is cleaved into $\mathrm{p} 48$, nucleoside triphosphatase (NTP), p22, viral genome-linked protein (VPg), and RNA-directed RNA polymerase (RdRp). The second ORF (ORF2) of $1.8 \mathrm{~kb}$ encodes VP1. The third ORF (ORF3) of about $0.6 \mathrm{~kb}$ encodes the $22 \mathrm{kDa}$ structural protein, VP2 (Donaldson et al., 2010).

The A-B dimer, formed from two capsid protein monomers, provides the receptor-binding region and sites of antigenic variation. The virus particle contains 180 monomers of the capsid protein assembled as dimers (Donaldson et al., 2010).

\subsubsection{Phenotypic Characteristics of Norovirus}

The norovirus capsid protects the genome from environmental degradation caused by increased temperatures, desiccation and nucleases, and confers a high degree of resistance to chemical disinfection compared to that observed with more complex viruses such as those with envelopes or bacteria (Wu et al., 2005). Norovirus particles are also resistant to other stressors such as moderate heat, chlorine and freezing temperatures (Nuermberger, 2005).

Because human noroviruses cannot be grown in cell culture, data on their environmental survival has been limited to studies with surrogate viruses such as feline calicivirus (FCV), murine norovirus (MNV-1) and other non-enveloped viruses such as bacteriophage MS2 (Weber et al., 2010). This limitation is relevant because these surrogate viruses may not accurately reflect how 
human norovirus behaves in regard to environmental survival and susceptibility to disinfectants (Weber et al., 2010). A number of studies have used human norovirus; however, analysis was done by monitoring damage to the genome using real-time reverse transcription quantitative polymerase chain reaction (RT-qPCR) and not infectivity (Hudson et al., 2007).

Norovirus in general can contaminate and survive in inanimate environments such as on surfaces and medical equipment (Hota, 2004). Norovirus survived in stool samples for $\leq 14$ days and $\leq 12$ days on carpets (through environmental swabbing and testing via RT-PCR) (Cheesbrough et al., 2000; Hota, 2004). Because norovirus is capable of surviving for long periods of time in the environment, contamination and the spread of norovirus via inanimate objects has been demonstrated by outbreaks on cruise ships (Nuermberger, 2005).

Human norovirus genome resisted complete degradation at $72{ }^{\circ} \mathrm{C}$ for 45 and 60 minutes (Weber et al., 2010). The virus can survive on surfaces of refrigerated foods for at least 10 days and in mineral and tap water for over two months at 4, 25 and $-20^{\circ} \mathrm{C}$ (Weber et al., 2010). FCV can survive on surfaces in a dried state for 21 to 28 days at room temperature (Weber et al., 2010). Norovirus can survive in a wide range of temperatures, from freezing to $60{ }^{\circ} \mathrm{C}$ and can survive on environmental surfaces and in a variety of foods (Weber et al., 2010). In a surface and ground water study, MNV-1 was a better human norovirus surrogate virus than FCV due to its genetic similarity and environmental stability (Bae \& Schwab, 2008). The infectivity reduction rates for FCV were significantly higher than other surrogate viruses at $25{ }^{\circ} \mathrm{C}$ in water, indicating that FCV is a much less stable surrogate virus than MNV-1 (Bae \& Schwab, 2008). A thermal inactivation study, which focused on the stability of FCV and human norovirus (GII.4 norovirus) 
RNA, demonstrated that human norovirus is heat stable when exposed for 2 minutes up to $76.6{ }^{\circ} \mathrm{C}$ compared to FCV which was only stable to $63.3{ }^{\circ} \mathrm{C}$ (Topping et al., 2009). MNV-1, FCV and bacteriophage MS2 survived in a variety of beverages such as juices, juice blends and milk at $4{ }^{\circ} \mathrm{C}$ (Horm \& D’Souza, 2011). MNV-1 showed no reduction in viral titer after 21 days in orange juice and milk but showed a moderate reduction of $1.4 \log$ in pomegranate juice, and was completely inactivated after 7 days in an orange and pomegranate juice blend (Horm \& D’Souza, 2011). FCV was completely inactivated after 14 days in orange juice and pomegranate juice; $~ 3 \operatorname{logs}$ of viral titer was inactivated after 21 days in milk, and the virus was completely inactivated after 1 day in an orange and pomegranate juice blend (Horm \& D’Souza, 2011). MS2 was inactivated by $\sim 1.28 \log$ and $<1 \log$ after 21 days in orange juice, and milk or pomegranate juice, respectively, and $<1 \log$ after 21 days in juice blends (Horm \& D'Souza, 2011). Human norovirus can survive on food preparation surfaces (such as stainless steel, Formica ${ }^{\circledR}$ and ceramic) and on human hands for long periods of time, and these can act as vehicles for norovirus transmission (Liu et al., 2009). In particular, norovirus survived after 2128 days of storage on surfaces and showed a gradual average reduction in detectable viral RNA ranging from 1.5 to $2.9 \log _{10}$ genome equivalent particles (GEP) through real-time RT-qPCR, whereas Snow Mountain virus showed greater environmental stability with 0.4 to $1.2 \log _{10}$ GEP reduction on all three types of surfaces after 42 days of storage (Liu et al., 2009).

Norovirus transmission through cleaning cloths (in particular, nonwoven cloths and cotton terry bar towels), which can transfer a significant amount of virus back to food contact surfaces, was documented by Gibson et al. (2012). Takahashi et al. (2011) demonstrated that the presence of food residues left behind on stainless steel surfaces from improper cleaning increased the 
survivability of MNV-1 and reduced the germicidal effect of sodium hypochlorite. The presence of organic material such as feces can generally decrease the efficacy of a number of disinfectants including organic acid, aldehyde, sodium hypochlorite and peroxide (Poschetto et al., 2007). A thermal inactivation study involving MNV-1, Hepatitis A virus (HAV) and FCV demonstrated that all three viruses were stable at $37{ }^{\circ} \mathrm{C}$ (Gibson \& Schwab, 2011). MNV-1 was statistically more stable than $\mathrm{FCV}$ at $50{ }^{\circ} \mathrm{C}$ (decimal reduction time [D-value] of MNV-1 was 106 minutes) whereas D-value of FCV was 50.6 minutes; however, HAV had significantly higher D-values than both MNV-1 and FCV at 50 and $60{ }^{\circ} \mathrm{C}$ with 385 and 74.6 minutes, respectively, suggesting that HAV was more resistant to thermal inactivation than both MNV-1 or FCV, with MNV-1 being moderately resistant and FCV being the least resistant (Gibson \& Schwab, 2011). The survivability of FCV was dependent on the type of fomite used, with the surrogate virus surviving on the surfaces of telephones for up to 3 days, computer mouse for 1 or 2 days, and computer keyboards and brass for 8 to 12 hours (Clay et al., 2006).

A comparison of the inactivation profiles of MNV-1 and FCV was conducted to determine the stability of the norovirus surrogate viruses upon exposure to $\mathrm{pH}$, organic solvents, temperature and environmental surface conditions (wet and dry conditions). MNV-1 was stable between $\mathrm{pH}$ 2 to 10 , with $<1 \log$ reduction at $\mathrm{pH} 2$, whereas $\mathrm{FCV}$ was inactivated at $\mathrm{pH}<3$ and $>9$. Both MNV-1 and FCV were inactivated at 63 and $72{ }^{\circ} \mathrm{C}$, however FCV was more stable than MNV-1 at $56^{\circ} \mathrm{C} . \mathrm{MNV}-1$ and FCV had shown long-term survival when both viruses were suspended in a fecal matrix and inoculated onto a stainless steel surface at $4{ }^{\circ} \mathrm{C}$; however, MNV-1 was more stable than FCV at room temperature. Overall, MNV-1 was selected to be a more suitable surrogate virus for human norovirus due to its ability to survive in gastric $\mathrm{pH}$ ranges and 
environmental conditions (Cannon et al., 2006). A comparison of the temperature, environmental and $\mathrm{pH}$ profiles of norovirus to other microorganisms from previous studies was compiled (Table 1).

\subsubsection{Norovirus Detection Methods}

Human noroviruses do not grow in cell culture so a definitive diagnosis of viral infection was initially accomplished by electron microscopy, an approach which proved to be relatively insensitive and could not be used for detecting low concentrations of virions found in food and water (Goodridge et al., 2004; Kapikian et al., 1972; Radford et al., 2004). Human challenge studies were also conducted to identify and diagnose norovirus infections (Donaldson et al., 2010). The use of enzyme linked immunosorbent assays (ELISA) for norovirus diagnosis is likewise not effective due to the antigenic variability of the capsid protein of different strains and its inherently lower sensitivity (Goodridge et al., 2004).

Currently, RT-PCR has become the standard for diagnosis of norovirus alone or as a component of multiplex RT-PCR assays which have been used to allow simultaneous detection of noroviruses, astroviruses and rotaviruses (Pang et al., 2005; Radford et al., 2004). However, a problem with the RT-PCR approach is that the genomic diversity of strains would mean that it is unlikely that a single primer pair would detect all strains over time, possibly leading to false negative results during testing (Goodridge et al., 2004). Another limitation with RT-PCR is that it does not indicate whether the virus is in its virulent, viable form, or if it is inactivated and poses no threat to human health (Richards, 1999). Because RT-PCR cannot discriminate between inactivated and infectious virus, false positives can occur; however, the detection of 
inactivated virus poses no risk to public health but may lead to unnecessary environmental disinfection and/or patient restrictions (Nuanualsuwan \& Cliver, 2002; United States Food and Drug Administration, 2012).

Because norovirus cannot be grown in cell culture, laboratories currently use either conventional or real-time RT-PCR assays to rapidly detect and diagnose the virus in specimens and samples in large numbers, especially during epidemics and endemic gastroenteritis (Duizer et al., 2004; Mattison et al., 2009; Patel et al., 2009). In addition to detection, the genotype frequency distribution or genotype profiles of norovirus strains derived from long-term norovirus strain collection can be used to differentiate between foodborne outbreaks that are caused by food contamination early in the food chain versus foodborne outbreaks that are caused by food handlers contaminating the food (Verhoef et al., 2010). This type of differentiation can be important to food safety authorities and public health institutions for estimating the number of foodborne outbreaks originating from different sources (Verhoef et al., 2010).

RT-PCR and nucleotide sequence analysis have been useful and promising approaches in molecular epidemiology studies to identify the source of infection and to differentiate between outbreaks that have been incorrectly assumed to be connected (Patel et al., 2009). The methods were used to sequence one or more regions of the virus genome through the use of primers by targeting regions of ORF1 or ORF2 originating respectively from either the polymerase or capsid (Gonin et al., 2000; Kageyama et al., 2003; Mattison et al., 2009; Vinjé et al., 2004). Because of the diversity of norovirus genomes, one of the challenges pertaining to the RT-PCR assay is 
finding the appropriate conserved sequences to use as PCR primers (Kageyama et al., 2003; Kojima et al., 2002).

Previous studies have used the RNA-dependent RNA polymerase region for amplification because it was believed to contain the most conserved nucleotide sequence in the norovirus genome; however, in contrast to the RNA-dependent RNA polymerase, the capsid N/S domain at the 5' end of the ORF2 is highly conserved within genogroup I or II (of noroviruses) with over $80 \%$ homology, whereas the ORF1-ORF2 junction region is the most conserved region of the norovirus genome (Kageyama et al., 2003; Katayama et al., 2002; Kojima et al., 2002). The major capsid protein (VP1) region D is now considered the reference genomic region for genotyping genogroup I and genogroup II norovirus strains (Vinjé et al., 2004). Most laboratories either sequence a small region of the polymerase (regions A and B) or the major capsid (regions C or D) gene to genotype strains (Gonin et al., 2000; Kojima et al., 2002; Mattison et al., 2009; Vinjé et al., 2003; Vinjé et al., 2004). In Europe, the FBVE network conducts surveillance for norovirus outbreaks and genotypes strains using a fragment of the RNA-dependent RNA polymerase at regions B, C and D (Verhoef et al., 2010).

Health Canada uses Kageyama primers (COG1F-COG1R) for the detection of genogroup I, Kageyama primers (COG2F and COG2R) for the detection of genogroup II, Monroe primers (431, 432, 433 and 434) for the detection of genogroups I and II, and Actin-A and Actin-R primers for the detection of oyster actin mRNA from genogroups I and II (Table 2) (Health Canada, 2010). The ORF1-ORF2 junction region showed that highly conserved nucleotide sequences were located at nucleotide 5279 to 5381 (102 bases) for Norwalk/68 virus (M87661) 
among norovirus genogroup I and nucleotide 4988 to 5108 (102 bases) for Camberwell virus (AF145896) among norovirus genogroup II (Kageyama et al., 2003). The method was performed using the Qiagen OneStep RT-PCR Kit (\#210210 or 210212) (Health Canada, 2010). The BCPHMRL (personal communication, April 5, 2011) currently uses Kageyama primers (COG1F and COG1R) and fluorescent probes (RING1(a)-TP and RING1(b)-TP) to detect norovirus genogroup I, and Kageyama primers (COG2F-COG2R) and fluorescent probe (RING2-TP) to detect genogroup II (Kageyama et al., 2003).

Clinical laboratories participating in CaliciNet, the US Centers for Disease Control's electronic norovirus outbreak surveillance network, use region D for genotyping noroviruses (Centers for Disease Control and Prevention, 2011c). Clinical diagnostic laboratories currently use real-time RT-PCR derived from Kageyama et al. (2003) which had been modified into one-step and duplex assays for high throughput screening and detection of genogroup I and II (Centers for Disease Control and Prevention, 2011c; Pang et al., 2005; Trujillo et al., 2006).

\subsubsection{Clinical Presentations of Norovirus Infections}

The human norovirus infection incubation period is between 12 and 48 hours with clinical symptoms being generally acute, and lasting between 1 to 2 days (Nuermberger, 2005; Radford et al., 2004). Symptoms include nausea, severe vomiting (most commonly projectile vomiting), watery diarrhea, abdominal cramps, headache, fever and general malaise (Hutson et al., 2004; Nuermberger, 2005; Radford et al., 2004). Diarrhea is usually non-inflammatory and most patients have no more than 3 to 4 watery stools per 24 hour period (Nuermberger, 2005). 
Norovirus has been shown to be present in the stool by RT-PCR for up to 2 weeks and although symptoms resolve, it is not clear if this virus continues to be infectious (Nuermberger, 2005).

Norovirus infection in the elderly or the immunocompromised can last longer and be more severe, and in some cases, have a fatal outcome (Radford et al., 2004). There are reports stating the potential for immunosuppressed patients to develop chronic disease from norovirus infection (Radford et al., 2004). The elderly and the chronically ill with gastroenteritis are also vulnerable to complications such as dehydration, electrolyte disturbances, and aspiration of vomitus (Wu et al., 2005).

Mattner et al. (2006) carried out a study which focused on high risk groups and clinical complications of norovirus infections. In particular, five outbreak wards, where 84 patients and 60 nurses were infected with the norovirus variant, Grimsby virus, were studied. Patients with cardiovascular disease and renal transplant were at a $20 \%$ greater risk for a fall in their potassium, elderly patients aged 65 and older had increased diarrhea lasting more than 2 days, and immunosuppression was a risk factor for creatinine increase of more than $10 \%$. Norovirus infections in patients with underlying conditions, such as cardiovascular disease, renal transplant and immunosuppressive therapy, may lead to severe consequences such as decreased potassium levels, increased levels of C-reactive protein and creatine phosphokinase (Mattner et al., 2006). It was concluded that such populations are at a higher risk of several clinical outcomes, especially patients with cardiovascular disease, those receiving immunosuppressive therapy, the elderly and organ transplant recipients. One study done in the 1970s by Schreiber et al. (1974) investigated intestinal biopsies of six human volunteers who orally ingested stool filtrate 
containing norovirus. The results showed volunteers exhibiting clinical gastroenteritis and the biopsies showed intestinal mucosal lesions with altered mucosal architecture, inflammation and abnormal absorptive cells.

\subsubsection{Epidemiology and Transmission of Norovirus}

Three main factors which contribute to the spread of norovirus infection include a large human reservoir that is susceptible to infection, a very low infectious dose, and the ability to be transmitted by a number of different routes (Lopman et al., 2003). Other factors include resistance to inactivation by commonly used disinfectants such as quaternary ammonium compounds and alcohols (Estes et al., 2006). Routes of norovirus transmission include personto-person transmission by fecal-oral route or aerosol formation from projectile vomiting, foodborne transmission and waterborne transmission (Lopman et al., 2003). Other modes of infection include indirect transmission from contaminated surfaces and consumption of contaminated foods and beverages (Zingg et al., 2005). Immunity is often short-term rather than long-term; long-term immunity appears difficult to maintain (Estes et al., 2006). Asymptomatic food handlers may contribute to foodborne outbreaks because the virus can be shed in stools for prolonged periods of time (Estes et al., 2006; Zingg et al., 2005).

Projectile vomiting from norovirus infection may distribute up to $3 \times 10^{7}$ virus particles as an aerosol (Barker et al., 2004). It has been estimated that a single incident of vomiting can generate between 300,000 and 3,000,000 infectious doses with an aerosol particle size of 25 to $30 \mathrm{~nm}$ (Zingg et al., 2005). Norovirus transmitted by fecal matter or vomitus from infected individuals may infect individuals ingesting as few as 10 to 100 virions (Caul, 1994; Hutson et 
al., 2004). An analysis of outbreaks from cruise ships demonstrated person-to-person transmission by showing that passengers were infected with the causative strain of norovirus even though they did not consume any contaminated food or drink (Nuermberger, 2005).

Consumption of norovirus-contaminated food is a significant mode of transmission for norovirus (Lopman et al., 2003, Zingg et al., 2005). Foods which serve as a vehicle of norovirus infection include oysters, salad vegetables, poultry, red meat, deli meats, fruit, soups, desserts and snacks (Lopman et al., 2003). In particular, consumption of raw and undercooked shellfish such as oysters has often been associated with outbreaks of gastroenteritis because shellfish are filter feeders capable of obtaining food, such as algae, by filtering small particles from the surrounding water and in some cases, they concentrate viruses and human pathogens derived from contaminated sewage water (Cheng et al., 2005; Lees, 2000).

Viruses documented to have been transmitted by the fecal-oral route associated with the consumption of bivalve shellfish include caliciviruses, astroviruses, rotaviruses, adenoviruses, enteroviruses and hepatitis A virus (HAV) (Lees, 2000). Other outbreaks associated with contaminated bivalve molluscan shellfish such as mussels, cockles, oysters and clams have also been documented (Lees, 2000; Tian et al., 2007). Oysters become contaminated with norovirus through bioaccumulation by the intestinal type A-like histo-blood group antigen (HBGA) (Tian et al., 2007). Three oyster species studied by Tian et al. (2007) expressed type A and type O-like HBGA in their gastrointestinal tissue, whereas mussels and clams were also found with similar type A-like antigens, and some only with O-like antigens. Multiple HBGAs are expressed in the gastrointestinal tissues of oysters, mussels and clams, allowing binding and bioaccumulation of 
norovirus to occur in these bivalve molluscan shellfish (Tian et al., 2007). The survival of FCV as a surrogate for norovirus in foods and on surfaces was investigated using plaque assay; infectious virus can survive in foods such as lettuce and ham, and on surfaces of stainless steel for up to 7 days (Mattison et al., 2007).

\subsubsection{Norovirus Outbreaks}

The CDC confirmed that 660 norovirus outbreaks occurred between 1994 and 2006 in the United States (Centers for Disease Control and Prevention, 2011c). Of the 660 laboratory-confirmed norovirus outbreaks, $35.4 \%$ originated from long-term care facilities, $31.1 \%$ from restaurants, parties and events, $20.5 \%$ from cruise ships and vacations, and $13.0 \%$ were from schools, child care and communities (Centers for Disease Control and Prevention, 2011c). The CDC also reported 1097 foodborne outbreaks in the United States during 2007, and within those outbreaks, there were 21,244 cases of foodborne illness and 18 deaths (Centers for Disease Control and Prevention, 2010). Of the 1097 foodborne outbreaks, 497 outbreaks (12,767 illnesses) were laboratory-confirmed with the etiologic agent identified; norovirus was the most common cause of foodborne outbreaks, followed by Salmonella (Centers for Disease Control and Prevention, 2010). Norovirus accounted for 193 outbreaks (39\%) out of the 497 laboratory-confirmed outbreaks (Centers for Disease Control and Prevention, 2010). In 2010, the National Enteric Surveillance Program of Canada reported 388 out of 3623 norovirus serotypes were found in British Columbia (Public Health Agency of Canada, 2010). Although norovirus transmission may occur year-round, norovirus outbreaks peaked during the winter months of the year in the northern hemisphere, especially in health-care institutions such as hospitals and residential homes (Lopman et al., 2003; Mounts et al., 2000). 
Norovirus has been the main cause of outbreaks in cruise ships, hospitals and hotels (Hota, 2004). Outbreaks in cruise ships have occurred on consecutive cruises despite attempts to disinfect and sanitize the ships between cruises (Hota, 2004). For ships that have been affected by outbreaks, discontinuation of services followed by vigorous and aggressive cleaning and sanitation of the vessels was required to stop the outbreaks (Hota, 2004).

Of the norovirus outbreaks from 1992 and 2000 in England and Wales, $40 \%$ occurred in hospitals and $39 \%$ occurred in residential-care facilities (Lopman et al., 2003). Norovirus infection which centred on elderly care and geriatric units occurred in $39 \%$ of hospital outbreaks and $89 \%$ of residential home outbreaks (Lopman et al., 2003). Hotels accounted for $7.8 \%$ of outbreaks, $4 \%$ occurred in schools, $6 \%$ were linked to food outlets, and $3.9 \%$ occurred in other settings such as private homes, holiday camps and military bases (Lopman et al., 2003).

In particular, residential-care facilities and hospitals facilitate the movement of viruses within the institutions because health care settings are semi-closed environments where patients and residents may experience person-to-person spread as well as being exposed to contaminated environments (Lopman et al., 2003). Norovirus outbreaks in health care settings are common and may affect vulnerable populations (Lopman et al., 2004). Hospitalized patients in health care facilities having norovirus infections may experience more severe outcomes and an increased duration of illness than other groups in the community (Lopman et al., 2004).

Calderon-Margalit et al. (2005) investigated six nursing homes in the Tel-Aviv district of Israel for three weeks in 2002 and found person-to-person transmission to be involved but social 
interaction was not attributed to the spread of the virus. Most of the nursing home residents were bedridden which suggested that the transmission of norovirus was from staff members to residents, possibly by direct contact (Calderon-Margalit et al., 2005). It was noted that the staff had encountered the virus either by direct contact from vomitus or feces or by indirect contact from contaminated surfaces (Calderon-Margalit et al., 2005).

Another study focused on the impact of hospital resources in norovirus outbreaks and found that attack rates were $13.9 \%$ among patients and $29.5 \%$ were among healthcare workers (Zingg et al., 2005). The financial impact of the outbreak on hospital resources, which comprised lost revenue, extra costs for diagnosis, increased nurse's care, and expenses for the infection control team, totalled \$40,675 USD (Zingg et al., 2005).

Norovirus infections have also occurred from the consumption of ready-to-eat meat prepared by infected food handlers (Malek et al., 2009). In 2005, a norovirus outbreak occurred among river rafters on trips on the Colorado River (Grand Canyon) (Malek et al., 2009). Of the 57 rafters who became ill at $\leq 72$ hours after the rafting trip began, $96 \%$ reported eating delicatessen meat originating from one batch purchased from one processing plant. The employee of the processing plant had sliced this batch with bare hands 1 day after recovering from gastroenteritis. Infected food handlers can contaminate ready-to-eat foods with norovirus during processing and handling, and it is important to include specific measures in meat-processing practices to prevent contamination and widespread outbreaks (Malek et al., 2009). 
There has been a sharp increase in norovirus outbreaks on cruise ships in the United States (Widdowson et al., 2004; Wikswo et al., 2011). In 2002, there were 14 laboratory-confirmed norovirus outbreaks on cruise ships of which 12 of the $14(86 \%)$ outbreaks were caused by caliciviruses (Widdowson et al., 2004). Eleven of the outbreaks were caused by noroviruses (Widdowson et al., 2004). Another study on a norovirus outbreak in a cruise ship has indicated that the outbreak affected passengers from two consecutive cruises and later continued on four following cruises despite a one week of sanitizing of the same ship (Isakbaeva, 2005). Epidemiological analysis suggests that an initial foodborne source of infection followed by secondary person-to-person transmission may have been the cause of the outbreak (Isakbaeva, 2005). Environmental contamination may have helped perpetrate the outbreak and infected crew members may have also contributed by being a reservoir of norovirus infection between cruises (Isakbaeva, 2005).

Over the past five years, an average of 27 norovirus outbreaks had been confirmed in cruise ships in the United States. In 2009, a norovirus outbreak on a cruise ship prompted an investigation, where it was found that person-to-person transmission (involving an incident of public vomiting) likely caused this high morbidity outbreak. A questionnaire was conducted in the retrospective cohort study on the cruise ship where the norovirus outbreak occurred. Of the 1842 passengers, $83.2 \%$ returned the questionnaire; the results revealed that over $90 \%$ of all passengers reported increased hand hygiene practices after becoming aware of the outbreak, and $38 \%$ of ill passengers and $11 \%$ of well passengers decreased their participation in public activities. The number of ill passengers who decreased their activities was low, indicating the 
need to encourage voluntary self-isolation for those who become ill and to report their illness to ship personnel (Wikswo et al., 2011).

\subsubsection{Susceptibility and Vaccine Development}

Because human noroviruses are a major cause of gastroenteritis throughout the world, an effective vaccine would be desirable in reducing morbidity and mortality in a population (Estes et al., 2000). There are many challenges in the development of a human norovirus vaccine: (1) immunity is not yet clearly defined and understood, (2) multiple strains and types of norovirus exist, (3) infection with one strain does not protect against subsequent infection with a different type of norovirus, (4) knowledge of mucosal immunity is limited, (5) norovirus cannot be grown in cell culture, and (6) no animal model for the infection exists (Estes et al., 2000). The genetic variability of noroviruses may confer a survival advantage against the immune response (Estes et al., 2006). It is not yet known whether the reason genetic variability is rapidly evolving because of the development of immunity against a specific strain of the virus, or if it is due to antigenic drift from errors during RNA polymerase replication, or both (Estes et al., 2006).

Norovirus has been found to be able to bind to gut-expressed carbohydrates leading to a correlation between a person's genetically determined carbohydrate expression and their susceptibility to norovirus infection (Hutson et al., 2004). HBGAs, complex glycans that are expressed on surfaces of red blood cells, gut and respiratory epithelia, and biological secretions in humans, appear to function as receptors or co-receptors for productive norovirus infections; however, there is no direct evidence to date that show viral binding to HBGAs lead to entry into host cells (Donaldson et al., 2010). 
Hutson et al. (2002) reported on the relationship between an individual's ABO histo-blood group type and the risk of norovirus infection and symptomatic disease after viral challenge by analysing serum samples from volunteers. Individuals with the group $\mathrm{O}$ phenotype were more likely to become infected with norovirus and symptomatic disease than individuals with the group B HBGA (Hutson et al., 2002). Individuals with blood group O are more susceptible to GI.1-NV infection than individuals with other blood groups (Donaldson et al., 2010). GI.1-NV binds to gastroduodenal epithelial cells from secretor-positive individuals, whereas the virus-like particle (VLP) did not bind to the cells of secretor-negative individuals (Donaldson et al., 2010). One study found that blood group and an individual's secretor status did not strongly correlate with susceptibility to viral infection; however, another study showed that secretor-negative individuals have lower antibody titer to GII.4 strains than secretor-positive individuals, suggesting that non-secretors are less likely to be infected than secretors (Donaldson et al., 2010; Marionneau et al., 2002; Thorven et al., 2005). These studies show that although norovirus strains may only infect a subset of the human population, the variability and the complexity of HBGA-binding affinities may cause nearly all individuals to be more susceptible to one or more strains (Donaldson et al., 2010). Norovirus strains have highly variable HBGA-binding patterns and as such, evolution of strains may be influenced by HBGA binding (Donaldson et al., 2010).

Short-term immunity (for up to 14 weeks) has been observed in previous volunteer challenge studies but this immunity did not extend to distinct antigenic strains of genetic clusters of norovirus, as volunteers from a virus challenge study who became ill with norovirus also became ill with another strain, Hawaii virus, at the same rate as volunteers who were not previously infected with norovirus (Matsui \& Greenberg, 2000; Wyatt et al., 1974). Generally, short-term 
immunity lasts between 6 and 14 weeks, and long-term immunity lasts between 9 and 15 months (Estes et al., 2000).

Previous rechallenge studies had shown that of all the volunteers who had been infected with norovirus and rechallenged with the same virus 27 to 42 months later, half of them became ill again with gastroenteritis with jejunal lesions, whereas the other half who were previously immune remained immune again with no jejunal lesions (Parrino et al., 1977).

An effective norovirus vaccine would be ideal for specific people in the population such as food handlers, care providers and military personnel (Lindesmith et al., 2005). However, there are many obstacles for norovirus vaccine development because of the large degree of antigenic heterogeneity and the rapid evolution of viruses within the family (Lindesmith et al., 2005). Also, there is a possibility that immunity against norovirus may be short-lived due to the variability and the growing list of norovirus strains (Goodridge et al., 2004; Lindesmith et al., 2005). Norovirus strains that are within a genetic cluster generally show $\geq 80 \%$ amino acid identity in the major capsid protein sequence, strains within the same genogroup show $\geq 60 \%$ identity, and strains that are in different genogroups show $\leq 50 \%$ identity (Lindesmith et al., 2005). Because of the high degree of genetic variability, there is a high degree of antigenic variability within noroviruses (Lindesmith et al., 2005).

A recent study by Atmar et al. (2011) was conducted to determine the safety, immunogenicity and effectiveness of an investigational norovirus VLP vaccine containing chitosan and monophosphoryl lipid A as adjuvants and delivered intranasally to healthy adult volunteers. 
Ninety-eight adult volunteers were enrolled in the study and randomly selected to receive two doses of either the vaccine or placebo, followed by inoculation with norovirus and monitored for viral infection and gastroenteritis symptoms. Of those vaccinated, $70 \%$ had an IgA response. Results showed that frequency of gastroenteritis was $69 \%$ for placebo recipients versus $37 \%$ for vaccine recipients, and frequency of norovirus infection was $82 \%$ for placebo recipients versus $61 \%$ for vaccine recipients, suggesting that norovirus vaccination had significantly reduced the frequency of gastroenteritis and virus infection and is capable of providing protection against norovirus illness and infection (Atmar et al., 2011).

\subsection{Prevention Strategies}

\subsubsection{Infection Control and Outbreak Prevention}

There are several measures that can be taken to minimize the spread of norovirus. Because viral particles can resist exposure including moderate heat, chlorine and freezing temperatures, to prevent secondary transmission of norovirus infection on cruise ship settings, careful attention must be put into infection control procedures including hand hygiene, barrier precautions such as gloves, and proper disposal and/or sterilization of materials that have been contaminated with the virus (Nuermberger, 2005). In addition, disinfection processes must include concentrated chlorine solutions, phenol-based compounds or accelerated hydrogen peroxide products (Nuermberger, 2005). Routine cleaning and disinfection of frequently touched environmental surfaces, equipment and clinical areas should be performed (MacCannell et al., 2011). Environmental surfaces that are frequently touched include toilets, faucets, hand and bed railings, telephones, door handles, computer equipment such as keyboards, and kitchen preparation surfaces (MacCannell et al., 2011). 
Barker et al. (2004) recommended that to achieve good hygiene, it is important that surfaces be wiped clean using a cloth soaked in detergent before applying a combined hypochlorite solution or detergent. Fingers contaminated with norovirus from fecal material could transfer the virus to up to seven clean surfaces. Despite using detergents and disinfectants, it has been shown that detergent-based cleaning with a cloth, even with a second wipe step, to produce a visibly clean surface failed to eliminate norovirus contamination, especially on surfaces contaminated with fecal soiling where norovirus could still be detected on $28 \%$ of surfaces, even with a combined hypochlorite or detergent formulation of 5000 ppm of available chlorine present (Barker et al., 2004).

Detergent-based cleaning alone or with a combined hypochlorite or detergent solution is not enough to eliminate norovirus contamination from surfaces and if the cleaning cloth is used to wipe another surface, viral transfer could occur from the cloth to another surface or to the hands of another person handling the cloth (Barker et al., 2004). For secondary contact surfaces that have become contaminated with norovirus either by transfer from hands or cloth, and where organic soiling is minimal, disinfection with bleach is sufficient to decontaminate surfaces and prevent the transfer of virus (Barker et al., 2004).

To maintain good hand hygiene during outbreaks, soap and water should be used after having contact with or providing care to patients with suspected or confirmed cases of norovirus (MacCannell et al., 2011). Food handlers must perform good hand hygiene practices before contact with or during preparation of foods and beverages (MacCannell et al., 2011). 
The CDC recommends avoiding exposure to bodily fluids such as vomitus and diarrhea from infected individuals during gastroenteritis outbreaks in healthcare settings (Centers for Disease Control and Prevention, 2011b). Patients infected with norovirus should go through longer periods of isolation and avoid contact with others for a minimum of 48 hours after symptoms resolve to prevent further exposure to other patients susceptible to the virus (Centers for Disease Control and Prevention, 2011b). Hand hygiene is recommended through the use of soap and clean water or ethanol-based hand sanitizers (60-95\% alcohol), and should be actively promoted among healthcare personnel, patients and visitors in patient care areas (Centers for Disease Control and Prevention, 2011b). Personal protective equipment (PPE) such as gowns and gloves are recommended when entering patient care areas to reduce exposure to vomitus or fecal matter (Centers for Disease Control and Prevention, 2011b). To avoid exposure and anticipated splashes from patients who are vomiting, eye protection or a full face shield is recommended (Centers for Disease Control and Prevention, 2011b). Soiled linens should be handed carefully as agitating them may allow the virus to be dispersed (MacCannell et al., 2011). Routine cleaning of frequently contaminated environmental surfaces and equipment such as commodes, toilets, faucets, sinks, hand and bed-railings, telephones, door handles, computer equipment and kitchen preparation areas is also recommended (Centers for Disease Control and Prevention, 2011b). The CDC recommends that chlorine bleach or United States Environmental Protection Agency (EPA)-approved products be used to clean and disinfect contaminated surfaces of patient care areas frequently, as much as twice daily to maintain cleanliness, and to clean and disinfect frequently touched environmental surfaces three times per day using EPA-approved products in healthcare settings (Centers for Disease Control and Prevention, 2011b; Centers for Disease Control and Prevention, 2011c). When using chlorine bleach solutions, the CDC recommends 
that they be used to disinfect hard, non-porous, environmental surfaces, and be prepared and used within 24 hours at concentrations of 1000-5000 ppm of chlorine (or 1:50 to 1:10 dilution of household bleach at $5.25 \%$ available chlorine) for at least a 4 minute contact time (Centers for Disease Control and Prevention, 2011c). Phenolic compounds, such as triclosan, and quaternary ammonium compounds are less effective against norovirus (Centers for Disease Control and Prevention, 2011c).

Guidelines for preventing gastroenteritis in child care programs recommend immediate diaperchanging practices, frequent hand-washing and exclusion of ill children from child group care facilities (Isakbaeva, 2005). Recommendations regarding norovirus outbreaks on cruise ships often include the implementation of basic measures such as hand washing, paid sick leave for ill workers, ongoing training for food handlers, and the isolation of people who are ill (Widdowson et al., 2004).

\subsubsection{Disinfectant Recommendations}

There are several studies with conflicting recommendations on what the appropriate hypochlorite concentration should be when used to disinfect contaminated surfaces or areas. The CDC recommends that chlorine-based disinfectants be prepared and used within 24 hours at concentrations of 1000-5000 ppm of chlorine (or 1:50 to 1:10 dilution of household bleach at $5.25 \%$ available chlorine) (Centers for Disease Control and Prevention, 2011c).

Recommendations from the norovirus control guidelines on cleaning vomit or fecal matter specify wearing appropriate disposable protective clothing such as gloves and an apron, using paper towels to soak up and remove any solid matter directly into a clinical waste bag, cleaning 
the contaminated area with detergent and hot water using a disposable cloth, disinfecting the contaminated area with $1000 \mathrm{ppm}(0.1 \%)$ hypochlorite solution, disposing the protective materials such as gloves and cloths into the clinical waste bag, and lastly washing hands thoroughly with soap and water (Chadwick et al, 2000). Barker et al. (2004) recommended that in order to achieve good hygiene, surfaces should be wiped clean using a cloth soaked with detergent before applying a hypochlorite solution. Hota (2004) has indicated that the decontamination of viruses requires $10 \%$ sodium hypochlorite solution (household bleach) or another biocide. In addition to using a germicidal product such as a $10 \%$ sodium hypochlorite solution (household bleach) for decontaminating areas affected with outbreaks, the closure of the affected institution or facility may also be required (Hota, 2004).

It is unknown at this time if alcohol-based hand sanitizers are effective against human norovirus (Centers for Disease Control and Prevention, 2011c). A finger pad method used for studying the effectiveness of liquid soap and hand sanitizers on norovirus showed that liquid soap (0.67 to $1.20 \log _{10}$ reduction) was more effective than alcohol-based hand sanitizers containing $62 \%$ ethyl alcohol (0.14 to $0.34 \log _{10}$ reduction) in reducing norovirus contamination on human hands (Liu et al., 2010). However, one limitation to the study by Liu et al. (2010) was that real-time RT-qPCR was used to determine the viral titer and $\log _{10}$ reduction of genomic copies of norovirus cDNA, even though RT-PCR is unable to differentiate between infectious and noninfectious virus (Baert et al., 2008). More research is needed to evaluate the efficacy of alcoholbased hand sanitizers against human or surrogates of human norovirus (MacCannell et al., 2011). At this time, it is recommended that ethanol-based hand sanitizers between 60 and $95 \%$ ethanol 
be used instead of other alcohol or non-alcohol-based hand sanitizers during outbreaks (Centers for Disease Control and Prevention, 2011b; MacCannell et al., 2011).

\subsection{Surrogate Viruses of Human Norovirus: MNV-1 and FCV}

One of the major problems with human noroviruses is that they do not grow in cell or organ cultures and to date, there is still no small animal model available which has made it difficult to study norovirus infection and gastroenteritis, and has made it difficult to study the efficacy of disinfectants (Ball et al., 1999; Hutson et al., 2004; Widdowson et al., 2004). Most knowledge of the natural history and other basic pathophysiological information about norovirus infections had been derived from outbreaks and volunteer studies (Hutson et al., 2004). Efforts to develop a methodology to cultivate human noroviruses in the laboratory were unsuccessful (Duizer et al., 2004). A recent study investigating the viral infection of a 3-dimensional (3D) model of the intestinal epithelium using Caco-2 cells with human norovirus has shown promise in developing an in vitro assay for human noroviruses; however, despite showing infection via shortening and loss of apical microvilli, viral enumeration was determined using real-time RT-qPCR but not plaque assay. Because plaque assay was not used in the study, viral RNA amplification may not necessarily point to the production of infectious virus particles (Straub et al., 2011). As a result, studies on norovirus persistence, inactivation and transmission have been limited to cultivable surrogate viruses such as FCV and MNV-1 (Cannon et al., 2006).

MNV-1 and FCV are norovirus surrogate viruses from the Caliciviridae family that are often used in experimental biomedical research for studying norovirus infection because of their ability to replicate in cell culture and animals (Wobus et al., 2004). 


\subsubsection{Murine Norovirus (MNV-1)}

MNV-1 was discovered in 2003; it was found to infect both wild-type and immunocompromised mice after peroral and intranasal inoculation, and was later found to also infect a significant number of laboratory mice nationwide (Wobus et al., 2004). MNV-1 is $28-35 \mathrm{~nm}$ in diameter, is icosahedral and its buoyant density is $1.36 \pm 0.04 \mathrm{~g} / \mathrm{cm}^{3}$ (Wobus et al., 2004). MNV-1 has the ability to infect the intestinal tract of mice through oral ingestion (Wobus et al., 2004). MNV-1 can also infect and grow in Kupffer cells (macrophages of the liver) and dendritic cells of mice (Wobus et al., 2004). Wobus et al. (2004) observed that MNV-1 formed plaques on murine cell line RAW 264.7 monolayers and showed for the first time that a norovirus can be cultivated in vitro. Hsu et al. (2007) investigated and isolated three novel MNV strains: MNV-2, MNV-3 and MNV-4. The three novel MNV strains differed from MNV-1 in pathogenicity because they produced persistent tissue infections in experimentally inoculated immunocompetent mice, whereas MNV-1 infection produced a transient infection which was typically cleared within the first week after inoculation (Hsu et al., 2007).

Because MNV-1 can replicate in murine macrophages and dendritic cells, human norovirus had been investigated to determine if it can also replicate in human macrophages and dendritic cells (Lay et al., 2010). Macrophage and dendritic cells isolated from peripheral blood mononuclear cells of individuals were exposed to norovirus, and analysed using immunofluorescence and RT-qPCR (Lay et al., 2010). The results had demonstrated that human norovirus does not replicate in human $\mathrm{CD} 11 \mathrm{c}^{+}$dendritic cells or monocyte-derived dendritic cells and macrophages (Lay et al., 2010). 
Detection of MNV-1 and FCV can be accomplished through RT-PCR directed to the ORF1/ORF2 junction region or more commonly through the plaque assay (Baert et al., 2008; Bidawid et al., 2003). The plaque assay is commonly used because this method enables quantification of virus titers through enumeration of viral plaques when infected RAW 264.7 monolayers of cells are maintained under agarose (Wobus et al., 2004; Bidawid et al., 2003). The RAW 264.7 macrophage mouse cell line was established from murine tumours induced by Abelson leukemia virus which was capable of expressing properties of macrophages (Raschke et al., 1978). In addition, the RAW 264.7 macrophage cell line was able to pinocytose neutral red dye at $1 \%$ (Raschke et al., 1978). New methods have been developed to detect MNV-1 although it is unknown if these methods will be favoured over the conventional plaque assay. Molecular beacons have been used for real-time monitoring of virus replication in living cells (Ganguli et al., 2011). The TAT peptide-delivered molecular beacon method for detecting and enumerating infective MNV-1 in RAW 264.7 cells was faster (by 12-fold) than the plaque assay (Ganguli et al., 2011). Baert et al. (2008) investigated both real-time RT-PCR and plaque assay method, and found that the quantification of genomic copies by real-time RT-PCR detected 100 times more virus than PFU by plaque assay. This may have been because the plaque assay only detects infectious virus while the RT-PCR detects viral RNA which may not be in an infectious virus particle (Baert et al., 2008).

MNV-1 is an effective enteric pathogen that is virulent in immunocompromised mice and infects wild-type mice (Wobus et al., 2004). Mice have been shown to shed infectious MNV-1 and transmit the virus from mouse to mouse in a cage or from contaminated bedding to uninfected mice (Wobus et al., 2004). Mice infected with the virus develop antibodies to MNV-1 within 3 
to 4 weeks of exposure (Wobus et al., 2004). MNV-1 strains can be isolated from laboratory mice feces or diarrhea (Wobus et al., 2004). The mouse virus, like the human norovirus strain, can be transmitted through the fecal-oral route, although at this time, it is uncertain if it can spread via the respiratory route, especially since the animals are not reported to experience vomiting (Wobus et al., 2004). MNV-1 can be inactivated at temperatures higher than $60{ }^{\circ} \mathrm{C}$ (Baert et al., 2008).

MNV-1 is classified under genogroup $\mathrm{V}$, and shares biological and molecular properties with other noroviruses and caliciviruses in general (Hsu et al., 2007; Wobus et al., 2004). In particular, MNV-1 shares biochemical and genetic features with human noroviruses, more specifically, its size, shape and buoyant density characteristics (Hsu et al., 2007; Karst, 2010). Compared to human norovirus (genogroup I), the nucleotide identities of the full length genome, after removing the gaps among humans and animal noroviruses, was at $52.4 \%$, demonstrating that although MNV-1 and human norovirus are related, they are distinct (Hsu et al., 2007). When MNV-1 was compared to MNV-2, MNV-3 and MNV-4, the related yet distinct strains of murine noroviruses ranged from $87.4 \%$ to $91.5 \%$ in identity (Hsu et al., 2007). Comparing fulllength nucleotide sequences for human norovirus strains and bovine norovirus of genogroup III, the MNV nucleotide sequences showed nucleotide identities ranging from $52.1 \%$ to $54.4 \%$ (Hsu et al., 2007).

The genome contains 7382 nucleotides (Hsu et al., 2007). The RNA genome begins with a 5' GU and ends with a poly-A tail (Hsu et al., 2007). Nucleotide position 33 of MNV-1 contains a C nucleotide instead of a U nucleotide as identified in other MNV strains (Hsu et al., 2007). 
MNV-1 genome has an ORF3 characteristic of noroviruses and vesiviruses, and two genera within the Caliciviridae family (Wobus et al., 2004). ORF1 of MNV-1 encodes a 187.5-kDa polyprotein containing $2 \mathrm{C}$ helicase, $3 \mathrm{C}$ protease and $3 \mathrm{D}$ polymerase motifs that are also found in other caliciviruses and picornaviruses; ORF2 encodes a 58.9-kDa capsid protein capable of selfassembling into virus-like particles when it is expressed in a baculovirus expression system, similar to caliciviruses; and ORF3 encodes a 22.1-kDA protein (Hsu et al., 2007; Wobus et al., 2004). ORF1 covers nucleotide positions 6 to 5069, ORF2 covers from 5056 to 6681, and ORF3 covers from 6681 to 7307 . For ORF1, the first AUG start codon begins at nucleotide position 6 with the second AUG beginning at nucleotide position 12, and the stop codon terminates ORF1 at nucleotide position 5069. Between ORF1 and ORF2, there is a 14-nucleotide overlap, creating a -2 frame shift of ORF2, and at the termination codon of ORF2 and the start codon of ORF3, there is a single nucleotide overlap which places ORF3 back to the same reading frame as ORF1. There is a 75 nucleotide 3' untranslated region before the polyadenylated tail at the end of ORF3 which contains a high A-U nucleotide content ranging from 66.7 to $69.7 \%$ (Hsu et al., 2007). Hsu et al. (2007) analysed the amino acid sequences of MNV-1 and found the polyprotein encoded by ORF1 contained 1,687 amino acids, the capsid protein encoded by ORF2 contained 541 amino acids, and the small structural protein encoded by ORF3 contained 208 amino acids. When MNV strains were compared to each other, amino acid identities coded for the polymerase gene ranged from 98.1 to $99.3 \%$, and the capsid gene ranged from 94.4 to 98.3\%. When compared to human and bovine noroviruses, the amino acid identities coded for the polymerase gene ranged from 51.3 to $54.7 \%$, and the capsid genes ranged from 37.8 to $41.3 \%$. When the four MNV strains were compared to each other, the amino acid identities for the S domain ranged from 99.1 to $100 \%$, whereas the $\mathrm{P}$ domain ranged from 90.8 to $97.5 \%$. 
When the MNV strains were compared to both human and bovine noroviruses, the amino acid identities for the S domain ranged from 53.6 to $58.2 \%$, whereas the P domain was less conserved and ranged from 25.4 to $32.8 \%$. Although the three novel murine noroviruses (MNV-2, MNV-3 and MNV-4) are related to MNV-1, they demonstrated a different pathogenicity from MNV-1, and are genetically distinct from MNV-1 and from one another, to justify their classification as unique MNV strains (Hsu et al., 2007).

It has been confirmed that a subgenomic RNA is expressed during virus replication and analysis via Northern blot from MNV-1-infected cells showed increasing amounts of subgenomic RNA over time (Wobus et al., 2004). Nucleotide sequences are conserved at the 5 ' end of the genome to the region just upstream of ORF2 which suggests that structural proteins of ORF2 and ORF3 are expressed from subgenomic RNA (Hsu et al., 2007; Wobus et al., 2004). Analysis of MNV-1-infected cells via electron microscopy showed changes in morphology including extensive reorganization of the intracellular membrane and the loss of the intact Golgi apparatus (Wobus et al., 2004). Observations show that norovirus replication may be associated with intracellular membranes although more research is needed to confirm this observation (Wobus et al., 2004).

\subsubsection{Feline Calicivirus (FCV)}

FCV was first isolated in tissue culture by Fastier (1957) and is present among cat populations worldwide (Ormerod \& Jarrett, 1978). The infection causes respiratory or systemic symptoms in cats, as opposed to gastrointestinal disease observed in infections with the human norovirus (Vashist et al., 2009). Infected cats can exhibit oral ulceration and/or mild upper respiratory 
disease, in addition to the less commonly occurring symptoms such as limping, abortion and severe pneumonia (Ossiboff et al., 2007; Pesavento et al., 2004). Infection in cats is not usually fatal; however, virulent, systemic strains of FCV can cause alopecia, cutaneous ulcers, subcutaneous edema and high mortality (Pesavento et al., 2004). Spontaneous virulent systemic FCV (VS-FCV) infection may produce lesions in cats through infection resulting in extensive facial and limb edema, ulcers on the skin, footpads, mouth, nares, tongue and pinnae, visceral and internal organ systems including lungs, liver, pancreas, spleen, and lymph nodes (Pesavento et al., 2004). Mature VS-FCV virus particles, which can be found within the cytoplasm of cell cultures, were unexpectedly discovered within the nuclei of epithelial cells (Pesavento et al., 2004). Although it is unclear at the moment, there is a possibility that physiologic breakdown of the nuclear envelope could be happening or that viral replication could be taking place within the nucleus; however, cells with virus present within their nucleus had no detectable cytoplasmic virus (Pesavento et al., 2004).

The rapid spread of FCV may be attributed to transmission through fomites and in small cat populations (Hurley et al., 2004; Ossiboff et al., 2007; Radford et al., 2006). Genetic diversity of FCV strains may be attributed to molecular substitution of immune-mediated positive selection and through endemically infected groups of cats which may allow new strains to evolve (Coyne et al., 2006; Radford et al., 2006). Shedding of FCV within cat colonies falls under three characteristics: persistent shedders, intermittent shedders and those resistant to infection (Radford et al., 2006). Shedding of the virus may occur as a result of persistent infection or by reinfection (Radford et al., 2006). 
Several vaccines are currently being used to vaccinate and protect cats against FCV, with vaccines being modified live, inactivated adjuvanted or inactivated non-adjuvanted. Intranasal vaccines are also available for cats in the United States. FCV vaccines are safe to use and have been shown to effectively reduce clinical disease in the cat population. Several different types of FCV strains have been selected for use in vaccines because of their broad cross-reactivity; however, most vaccines used are based on one strain, in particular, the FCV F9 or FCV 255 strain. One important limitation with FCV vaccines is that it does not protect against infection and it is possible that vaccinated cats may become infected with field strains without exhibiting any clinical signs or symptoms (Radford et al., 2006).

A comparison of amino acid sequences between FCV field isolates from Japan and global isolates revealed that FCV strains generally fall into two distinct groups: genogroup I and II (Sato et al., 2002). Genogroup I included $33 \%$ of Japanese strains and most of the global isolates, and may be considered genetically identical; however, genogroup II was comprised of $67 \%$ of Japanese isolates and no global isolates, indicating that the Japanese lineage is only confined to Japan (Ossiboff et al., 2007; Sato et al., 2002). Recombination of FCV is possible in cats infected with more than one strain of virus at the same time, with the recombinant virus containing elements of both strains circulating in the infected cats (Coyne et al., 2006).

The in vitro growth properties of various FCV isolates such as FCV F9 (vaccine strain), VS-FCV and non-VS-FCV clinical isolates were investigated, and the results showed that the growth kinetics of the FCV isolates differing in virulence share common properties such as rapid growth during a single round of virus replication, but may differ in virus production. Virulent FCV 
isolates tend to produce larger plaques and grow faster than less virulent isolates. VS-FCV isolates produced earlier morphological changes at 14 hours post-infection including rounding up and detaching partially or fully from the plate surface, whereas morphological alterations were not present in cell cultures with most non-VS isolates, suggesting that cytopathic effects may be correlated with virulence. Thermal resistance varied among all FCV isolates tested, with all FCV isolates losing substantial infectivity following 30 minutes incubation at $46.2^{\circ} \mathrm{C}$; however, the FCV F9 vaccine strain was most sensitive to thermal inactivation, losing all infectivity following a 30 minute incubation period at $52.2^{\circ} \mathrm{C}$. Other isolates were completely inactivated at $56.9^{\circ} \mathrm{C}$ except for the FCV-5 strain which was inactivated only at $62{ }^{\circ} \mathrm{C}$. Environmental stability at room temperature after 3 days varied among all FCV isolates, with most isolates losing 2-5 $\log _{10}$ infectivity, two VS-FCV isolates (Deuce and FCV-5) losing 2-3 $\log _{10}$ infectivity, and FCV F9 strain losing infectivity by $4-5 \log _{10}$. None of the FCV isolates lost infectivity after 8 weeks at $-80^{\circ} \mathrm{C}$. There was no correlation between virulence and temperature inactivation for the isolates tested (Ossiboff et al., 2007). Even though the F9 vaccine strain has been used extensively to investigate the sensitivities and environmental inactivation of caliciviruses, FCV field strains may be more resistant to temperature inactivation than the F9 strain (Duizer et al., 2004; Ossiboff et al., 2007).

Because FCV has shown good growth in vitro, it has been used as a surrogate virus for human norovirus studies (Vashist et al., 2009). The plaque assay method using feline embryo-derived cells has been used for FCV studies because virus plaque formation in agarose does not show inhibition and can produce clear, cytopathic effects (Bidawid et al., 2003; Ormerod \& Jarrett, 1978). The tissue culture infectious dose $\left(\mathrm{TCID}_{50}\right)$ method has also been used to quantify the 
infectivity of FCV using Crandell Reese feline kidney cells (CRFK) as the established cell line (Doultree et al., 1999; Bidawid et al., 2003).

FCV isolates may vary in terms of serum cross-neutralization and amino acid sequence in the capsid protein, in addition to plaque morphology (Ormerod \& Jarrett, 1978; Seal et al., 1993). Seal et al. (1993) analysed three FCV isolates, NADC, KCD and CFI/68, and found the capsid protein amino acid sequences to have 89 to $91 \%$ similarity, while nucleotide sequence data showed genetic variability among the three isolates (Seal et al., 1993). The amino acid sequences showed two regions of sequence divergence with variation as much as $55 \%$, which suggests that polyvalent vaccines may offer more protection than monovalent vaccines (Seal et al., 1993). The mechanism behind FCV infection of CRFK cells is unclear; however, it involves a low pH-dependent step (Kreutz \& Seal, 1995). Chloroquine, a lysosomotropic agent involved in the prevention of replication of non-enveloped viruses through acidification of intracellular vesicles, inhibited the production of FCV in the initial stages of viral adsorption and replication but had no effect when the agent was added 3-4 hours post-infection, suggesting that FCV enters cells through receptor-mediated endocytosis and requires a low $\mathrm{pH}$-dependent viral uncoating step (Kreutz \& Seal, 1995).

The FCV genome contains four single-stranded RNAs of 8.2, 4.8, 4.2 and $2.4 \mathrm{~kb}$ (Neill \& Mengeling, 1988). The genome of FCV strain F9 is 7690 bases long and contains two large ORFs (Carter et al., 1992). FCV encodes non-structural proteins at ORF1 from the 5' end of the RNA and capsid proteins from ORF2 at the 3' end (Carter et al., 1992). ORF1 extends from positions 2 and 5308, and has the first ATG codon occurring at position 20 (Carter et al., 1992). 
ORF2 extends from positions 5314 and 7326, and specifies a 671 amino acid protein (Carter et al., 1992). A short ORF3 of 318 bases at the 3' end of the virus RNA extends from positions 7326 and 7643, and specifies a 106 amino acid protein (Carter et al., 1992). Analysis via Northern blotting indicates that viral RNAs are nested, co-terminal transcripts with common 3' ends, with the transcript beginning at specific points and continuing to the 3' end of the FCV genome (Neill \& Mengeling, 1988). The $2.4 \mathrm{~kb}$ subgenomic RNA encodes the single capsid protein and contains 2 ORFs (Neill et al., 1991). The larger ORF from the 5' end of the subgenomic mRNA consists of 2004 bases and encodes a polypeptide with a mass of 73,467 Da (Neill et al., 1991). The smaller ORF located in the 3' end of the mRNA is made up of 318 bases and encodes a polypeptide with a mass of $12,185 \mathrm{Da}$ (Neill et al., 1991). The UGA termination codon of the larger ORF overlaps with the AUG initiation codon of the smaller ORF and resembles the -1 frameshift sequences such as that seen in retroviruses or coronaviruses (Neill et al., 1991). Western immunoblot analysis using feline anti-FCV antiserum revealed that translation of the capsid protein was detectable at 3 to 8 hours post-infection (Neill et al., 1991).

Some strains of low virulence FCV produced large amounts of defective low density virus particles known as defective interfering particles, while more virulent strains produced few to no defective virus particles. RNA of $2.4 \mathrm{~kb}$ isolated from low density virus particles encodes the capsid protein. The 5' end, mapped via primer extension, had the same genomic location as that of intracellular $2.4 \mathrm{~kb}$ RNA. Lower density viral particles had a density of $1.35 \mathrm{~g} / \mathrm{cm}^{3}$ whereas the infectious wild-type virus particle had a density of $1.39 \mathrm{~g} / \mathrm{cm}^{3}$, suggesting that lower density particles contain FCV subgenomic RNA instead of a genomic RNA containing rearrangements or deletions. It is not understood how or why lower density particles interfere with viral 
infection and replication; however, a possible explanation could be that lower density particles may interfere by binding to available cellular receptors and competing with the binding and uptake of complete, infectious virus particles; or viral resources could be drained during encapsidation of subgenomic RNA leading to the removal of a significant amount of translational template and capsid protein, thereby decreasing capsid production and infectious virus production (Neill, 2002).

\subsection{Disinfectants and Cleaning Agents}

Norovirus contaminated surfaces in health care facilities, cruise ships and daycare facilities must be treated to eliminate the virus. This often involves first cleaning the surface and then inactivating the virus particles using chemical agents. Disinfection and sterilization are two major categories of methods for inactivating viruses (Hota, 2004). Sterilization is the complete elimination of all microbial life on an object or surface and usually involves the application of physical or chemical means such as heat or chemical methods (Hota, 2004; Rutala \& Weber, 1997). Disinfection eliminates most microbes, except for bacterial spores, and usually involves the use of chemical agents (Hota, 2004). Biocides are chemical agents that are most often considered broad spectrum and vary in terms of antibacterial activity (McDonnell \& Russell, 1999). Biocides can inactivate or inhibit the growth of microorganisms in or on living tissue, and are often referred to as "-static", denoting inhibition of the growth of a variety of organisms including bacteria, fungi and spores (e.g., bacteriostatic, fungistatic and sporistatic), and as “-cidal”, denoting killing of bacteria, spores and viruses (e.g., bactericidal, sporicidal, virucidal) (McDonnell \& Russell, 1999). Disinfectants are generally defined as chemical products or biocides that can be used to inactivate microorganisms on inanimate objects or surfaces; 
however, they can be sporostatic but not necessarily sporicidal (McDonnell \& Russell, 1999). Sanitizers, on the other hand, are substances which reduce but do not necessarily eliminate all microorganisms and contaminants, especially vegetative cells, on surfaces to levels are that considered safe to the public (Marriott \& Gravani, 2006). Cleaning is the process of removing foreign material from surfaces or objects and often involves both mechanical processes and the use of detergents with water (Hota, 2004). Detergents will remove organic material and suspend grease or oil from surfaces (Hota, 2004).

Three different approaches can be used for cleaning contaminated surfaces: detergents, disinfectants and detergent-disinfectants (Hota, 2004). Disinfectants are agents which rapidly kill or inactivate many or all infectious particles with the exception of bacterial endospores (Hota, 2004; Rutala \& Weber, 1997). Detergent-disinfectants have the ability of achieving the aims of both detergents and disinfectants (Hota, 2004). Despite a lack of conclusive evidence to show that routine disinfection of hospital surfaces is better than the use of detergents alone, the routine use of detergent-disinfectants is common and is based on consensus and logistic considerations (Hota, 2004). The effectiveness of the disinfectants may be dependent on a number of factors including prior cleaning of the surface, presence of organic material, the type and level of contamination, concentration of the biocide, exposure time, type or nature of the surface or object, temperature, and pH of the biocide (Rutala \& Weber, 1997).

Disinfectants may be divided according to their efficacy. Chemical sterilants are known to kill endospores after long exposure times of between 6 to 10 hours. High-level disinfectants can kill and inactivate all microorganisms with the exception of endospores at exposure times of less 
than 45 minutes. Intermediate-level disinfectants may kill vegetative bacteria, most viruses and fungi, with the exception of endospores. Low-level disinfectants can kill most vegetative bacteria, and some fungi and viruses at short exposure times of less than 10 minutes, but are unable to kill endospores. Antiseptics, on the other hand, can prevent or arrest the growth of microorganisms and are often used to disinfect preparations involved in the application on living tissue such as skin (Rutala \& Weber, 1997).

In hospitals, patient rooms should be cleaned daily and subjected to terminal cleaning after a patient has been discharged from the hospital (Hota, 2004). Terminal cleaning involves thorough cleaning of non-critical, inanimate surfaces in the environment with a disinfectant, typically a quaternary ammonium compound or phenolics, and is more effective in de-germing the environment due to its greater thoroughness (Hota, 2004).

\subsubsection{Chlorine Compounds}

Halogen-releasing agents such as chlorine dioxide, chloramines-T and sodium hypochlorite are commonly used as high-level disinfectants in hospitals to disinfect certain types of equipment, and as a low-level disinfectant to disinfect non-critical environmental surfaces (McDonnell \& Russell, 1999; Rutala \& Weber, 1997). They are highly active oxidizing agents and can destroy cellular activity of proteins (McDonnell \& Russell, 1999). Hypochlorite agents are used as broad spectrum antimicrobial disinfectants and contain properties such as rapid bactericidal action,

persistence, especially in treating potable water, solubility in water, stability at its concentrated and diluted form, relatively non-toxic at recommended concentrations, and ease of use (Rutala \& Weber, 1997). 
Hypochlorite is also a deodorizer, is colourless, non-flammable, non-staining, and is relatively cheap to purchase (Rutala \& Weber, 1997). In water, sodium hypochlorite ionizes to produce sodium ion $\left(\mathrm{Na}^{+}\right)$and hypochlorite ion $\left(\mathrm{OCl}^{-}\right)$, resulting in an equilibrium with hypochlorous acid (HOCl) (McDonnell \& Russell, 1999). Chlorine is predominantly present as $\mathrm{HOCl}$ at $\mathrm{pH} 4$ to 7 and as $\mathrm{OCl}^{-}$at $\mathrm{pH} 9$ and above (McDonnell \& Russell, 1999). $\mathrm{HOCl}$ is the antimicrobial component of hypochlorites (McDonnell \& Russell, 1999). Chlorine-based compounds at higher concentrations are sporicidal, causing the spore coat to separate from the cortex, resulting in lysis; however, this is dependent on the concentration of the disinfectant and $\mathrm{pH}$ (McDonnell \& Russell, 1999). Hypochlorites are also virucidal and can inactivate viruses by degrading RNA (McDonnell \& Russell, 1999). Disadvantages of using hypochlorite disinfectants include irritation to mucous membranes, formation of toxic chlorine gas when coming into contact with certain chemicals, noticeable odour when used at high concentrations, decreased efficacy in the presence of a soil or organic load, and corrosive effects on certain metals and irritation of human skin (Rutala \& Weber, 1997). Another disadvantage is the formation of organohalides which are formed by the reaction of chlorine and organic compounds present in water and wastewater (Rutala \& Weber, 1997). These halogenated organic compounds known as trihalomethanes (e.g., chloroform) have been detected in chlorinated water and are a potential health concern (Rutala \& Weber, 1997).

\subsubsection{Quaternary Ammonium Compounds}

Quaternary ammonium compounds (QUATs) are membrane-active agents and amphoteric surfactants that are frequently used on clinical and industrial environments such as floors, walls, furnishings and equipment (Marriott \& Gravani, 2006; McBain et al., 2004; McDonnell \& 
Russell, 1999). They include alkyldimethylbenzylammonium chloride and alkyldimethylethylbenzylammonium chloride which are effective in water at 500 to $1000 \mathrm{ppm}$ hardness without the presence of sequestering agents (Marriott \& Gravani, 2006). Other QUATs include diisobutylphenoxyethoxyethyl dimethyl benzyl ammonium chloride and methyldodecylbenzyltrimethyl ammonium chloride which require high dilution for germicidal or bacteriostatic action (Marriott \& Gravani, 2006). Benzalkonium chloride is another QUAT that has been increasingly used in domestic cleaning products (McBain et al., 2004). QUATs are surface-active agents because they are wetting agents with built-in detergent properties and can penetrate porous surfaces (Marriott \& Gravani, 2006). The molecular structure of a surfactant consists of one hydrocarbon, hydrophobic group, and a hydrophilic polar group (McDonnell \& Russell, 1999). Surfactants can be cationic, anionic, non-ionic or ampholytic (amphoteric) compounds, depending on the charge or absence of ionization of the hydrophilic group (McDonnell \& Russell, 1999). QUATs are known to reduce mould growth and Listeria monocytogenes but are sporostatic, inhibiting the growth of spores but not the germination process (Marriott \& Gravani, 2006; McDonnell \& Russell, 1999). They are known to inhibit the growth of bacterial spores but not kill spores (Marriott \& Gravani, 2006). They also have an effect on enveloped viruses such as human immunodeficiency virus (HIV) but not on nonenveloped viruses (McDonnell \& Russell, 1999). QUATs contain surfactant properties which cause them to foam, and when formulated with a specific detergent, they can be used as a cleaner-sanitizer, making them appropriate for use in bathrooms, toilets, locker rooms and other non-food contact surfaces, as long as a rinsing step is involved (Marriott \& Gravani, 2006). They are effective in the alkaline $\mathrm{pH}$ range, are non-corrosive and non-irritating to the skin, nontoxic, colourless and odourless, stable in the presence of organic matter, and have a long shelf 
life (Marriott \& Gravani, 2006). Gram-negative bacteria are more susceptible to QUATs in the acid $\mathrm{pH}$ range and Gram-positive bacteria are more susceptible to QUATs in the alkaline range (Marriott \& Gravani, 2006). QUATs can damage the outer membrane of Gram-negative bacteria, allowing for their uptake (McDonnell \& Russell, 1999). The quaternary ammonium compound consists of four organic groups that are linked to a nitrogen atom that produces a positively charged ion called a cation whereas chloride is usually the anion (Marriott \& Gravani, 2006). It is speculated that the mechanism of germicidal action involves surface-active properties of the QUAT, in particular, electrostatic ionic interactions with phospholipids, by surrounding and damaging the cytoplasmic membrane lipid bilayers of the cell through the positively charged quaternary nitrogen, causing the wall to fail, resulting in leakage of the cytoplasmic materials, and inhibition of enzymes (Denyer \& Stewart, 1998; Marriott \& Gravani, 2006; McBain et al., 2004; McDonnell \& Russell, 1999). When QUATs are applied to surfaces for disinfection, they form a residual antimicrobial film that is bacteriostatic (Marriott \& Gravani, 2006). QUATs are stable in the presence of organic matter but their effectiveness decreases in their presence; however, the effectiveness of QUATs is not affected by hard water (Marriott \& Gravani, 2006).

\subsubsection{Alcohols}

Alcohols are rapid broad-spectrum disinfectants which can be used against vegetative bacteria, mycobacteria, viruses and fungi but not spores (McDonnell \& Russell, 1999). Alcohols such as ethanol and propanol work by targeting membrane integrity resulting in cell leakage, with the mechanism of interaction involving phospholipids (Denyer \& Stewart, 1998). Alcohols such as ethyl alcohol (e.g., ethanol, alcohol), isopropyl alcohol (e.g., isopropanol, propan-2-ol) and 
$n$-propanol are the most widely used disinfectants, with $n$-propanol being more commonly used in Europe (McDonnell \& Russell, 1999). They are known to inhibit sporulation and germination of spores (McDonnell \& Russell, 1999). Alcohols are not often used for sterilization because of the inability to kill spores, hence they are not considered sporicidal (McDonnell \& Russell, 1999). Isopropyl alcohol is slightly more effective against bacteria and ethyl alcohol is more effective against viruses but the efficacy of the alcohols is dependent on their concentration and the organism (McDonnell \& Russell, 1999). The antimicrobial activity is optimal between 60 and $90 \%$ and slightly lower at concentrations below $50 \%$ (McDonnell \& Russell, 1999).

\subsubsection{Hydrogen Peroxide}

Hydrogen peroxide is a disinfectant, antibacterial agent and oxidizing agent that is widely used for disinfection, sterilization and antisepsis (Denyer \& Stewart, 1998; McDonnell \& Russell, 1999). The biocide targets protein thiol groups, resulting in DNA strand breakage, and has been known to be effective against biofilms (Denyer \& Stewart, 1998; Marriott \& Gravani, 2006; McDonnell \& Russell, 1999). This disinfectant can be used on all types of surfaces, equipment, floors, drains, walls and other contaminated areas (Marriott \& Gravani, 2006). The mechanism involves free-radical oxidation leading to the formation of free hydroxyl radicals, which attack cell components such as lipids, proteins and DNA (Denyer \& Stewart, 1998; McDonnell \& Russell, 1999). Hydrogen peroxide oxidizes and disrupts thiol groups in enzymes and proteins (McDonnell \& Russell, 1999). Hydrogen peroxide is effective against viruses, bacteria, yeasts and bacterial spores (McDonnell \& Russell, 1999). In the food industry, hydrogen peroxide has been used for the sterilization of food packaging materials (Marriott \& Gravani, 2006). It is environmentally friendly because it degrades into water and oxygen (McDonnell \& Russell, 
1999). Hydrogen peroxide differs from accelerated hydrogen peroxide (AHP). AHP is a patented disinfectant containing a synergistic blend of ingredients that, when combined with low levels of hydrogen peroxide, increases its germicidal potency and cleaning performance (Virox Technologies Inc., 2011). AHP is made up of a number of ingredients including hydrogen peroxide, acids, surfactants, wetting agents and chelating agents (Virox Technologies Inc., 2011).

\subsubsection{Chlorhexidine}

Chlorhexidines are broad-spectrum disinfectants most commonly used in antiseptic products, in particular hand washing and oral products, which target the cytoplasmic membrane of cells; however, they are not sporicidal (McDonnell \& Russell, 1999). Low concentrations of chlorhexidine can affect membrane integrity, whereas at high concentrations, this can cause congealing of the cytoplasm through coagulation of intracellular constituents (McDonnell \& Russell, 1999). It is $\mathrm{pH}$ dependent and its efficacy can be greatly reduced in the presence of organic matter (McDonnell \& Russell, 1999). Chlorhexidine can cross the cell wall or outer membrane through passive diffusion and target bacterial cytoplasmic or inner membrane, leading to leakage of intracellular constituents and cell death (McDonnell \& Russell, 1999).

\subsubsection{Phenolic Compounds}

Phenolic-type compounds are disinfectants and antimicrobial agents which target the cytoplasmic inner membrane of cells causing leakage and occasionally uncoupling (McDonnell \& Russell, 1999). This leakage results in the release of intracellular constituents including potassium ions $\left(\mathrm{K}^{+}\right)($McDonnell \& Russell, 1999). Phenolic compounds possess antifungal and 
antiviral properties by damaging the plasma membrane of fungi and causing leakage (McDonnell \& Russell, 1999). Hydroxytyrosol, a phenolic compound, has been shown to inactivate enveloped viruses such as influenza but not non-enveloped viruses such as bovine rotavirus and fowl adenovirus (Yamada et al., 2009). The findings suggest that the mechanism behind the antiviral effect of phenolic compounds may require the presence of a viral envelope (Yamada et al., 2009).

\subsection{Disinfectant Studies on Surrogate Viruses: MNV-1 and FCV}

A number of studies done on surrogates of norovirus focused on the efficacy of disinfectants (Table 3). FCV, in particular, has been commonly used as a surrogate to investigate the survival, persistence and inactivation of human norovirus.

A number of studies focused on the efficacy of disinfectants on a number of surfaces including stainless steel. Frank \& Chmielewski (1997) demonstrated that surface materials have inherently different properties when being sanitized, and that the efficacy of the sanitizer was not a function of roughness or porosity. The efficacy of disinfectants on a number of different surface materials, which included mechanically polished or electropolished stainless steel, polycarbonate and mineral resin, was investigated. The surface materials were inoculated with Staphylococcus aureus at $10^{4}$ to $10^{5} \mathrm{CFU} / \mathrm{cm}^{2}$, immersed into sanitizer solution and then wiped with a sanitizersaturated cloth. Stainless steel and smooth polycarbonate surfaces were more readily sanitized by QUATs than mineral resin and abraded polycarbonate surfaces (Frank \& Chmielewski, 1997). 
Chlorine was most effective on mechanically polished and un-abraded electropolished stainless steel, and polycarbonate surfaces, but was less effective on abraded electropolished stainless steel and mineral resin surfaces. It is not known why abraded electropolished stainless steel surfaces maintained higher residual levels of viable cells than mechanically polished stainless steel surfaces after QUAT and chlorine treatment; however, abrasion of mechanically polished stainless steel had no effect on the efficacy of the sanitizer. More research on the relationship between surface roughness and stainless steel cleaning and sanitizing is needed (Frank \& Chmielewski, 1997).

Urakami et al. (2007) tested the sensitivity of FCV to chlorine by using a suspension of FCV and treating it with sodium hypochlorite containing $10 \mu \mathrm{g} / \mathrm{mL}$ of free chlorine. The study showed that more than $4.6 \log$ of partially purified FCV could be inactivated with $300 \mathrm{ng}$ free chlorine $/ \mathrm{mL}$ when cells were monitored for cytopathic effects in 96-well multiplates containing CRFK cells using the TCID 50 method (Urakami et al., 2007). Jimenez \& Chiang (2006) studied the virucidal activity of R-82, a QUAT disinfectant, on hard surface carriers containing dried FCV suspension with an organic soil load of $5 \%$ fetal bovine serum (FBS). After a 10 minute contact time at 1:256 dilution $(850 \mathrm{ppm})$, the formulation caused a 6.6 and $6.4 \log _{10}$ reduction and the virus was completely inactivated (Jimenez \& Chiang, 2006).

Omidbakhsh \& Sattar (2006) investigated the effectiveness of a new accelerated hydrogen peroxide-based surface disinfectant product, Accel TB, which contained $0.5 \%$ AHP, on selected microorganisms and non-enveloped viruses such as FCV strain F9 (VR-782) under dry load conditions containing a $5 \%$ FBS soil load. When AHP was used at $20^{\circ} \mathrm{C}$ at full strength 
concentration, it was bactericidal and virucidal in 1 minute, and mycobactericidal and fungicidal in 5 minutes. There was $>6.25 \log _{10}$ reduction of FCV after 1 minute contact time (Omidbakhsh \& Sattar, 2006).

Hand disinfection studies have also been done on surrogate viruses. Gehrke et al. (2004) examined ethanol, 1-propanol and 2-propanol on FCV inactivation with artificially contaminated fingertips using quantitative end-point titration method (50\% $\mathrm{TCID}_{50}$ method), and found that 1-propanol was more effective than ethanol and 2-propanol in inactivating FCV. Results from suspension tests without the use of contaminated fingertips showed a 4.13 and $4.06 \log _{10}$ reduction in virus with 1-propanol after a 30 second contact time at $50 \%$ and $70 \%$ concentrations, respectively, as opposed to the other 2 alcohols which were effective with $>4$ $\log _{10}$ reduction after a 3 minute contact time at 50 and $70 \%$ concentration (Gehrke et al., 2004). Results from tests using contaminated fingertips showed significantly higher reductions at $70 \%$ concentration for 1-propanol, ethanol and 2-propanol, with 3.58, 3.78 and $2.15 \log _{10}$ reduction after 30 seconds, compared to results at $90 \%$ concentration, which produced $\log _{10}$ reductions of 1.38, 2.84 and 0.76, respectively (Gehrke et al., 2004). Gehrke et al. (2004) concluded that ethanol and 1-propanol-based solutions with an alcohol concentration of $70 \%$ were most effective, and $80 \%$ solution of 2-propanol was least effective with $2.38 \log _{10}$ reduction after 5 minutes. Alcohols such as $50 \%$ 2-propanol, $50 \%$ ethanol and $35 \%$ methyl alcohol were ineffective against FCV, whereas phenolics, Clorox, aldehydes and creolin inactivated the surrogate virus (Steinmann, 2004). 
Lages et al. (2008) investigated the virucidal efficacy of nine sanitizers, four of which were alcohol-based (99.5\% ethanol, $62 \%$ ethanol, $91 \%$ isopropanol, $70 \%$ isopropanol), three were non-alcohol-based (3\% hydrogen peroxide, $0.13 \%$ benzalkonium chloride, $10 \%$ povidoneiodine) and two were triclosan-based antimicrobial liquid soaps ( $0.60 \%$ triclosan, $0.115 \%$ triclosan), against FCV on artificially contaminated fingertips for 30 seconds and 2 minute contact times. The virus was serially diluted and titrated in 96-well microtiter plates containing CRFK monolayers of cells, and observed for cytopathic effects using $50 \%$ TCID $_{50}$ method. The $99.5 \%$ ethanol-based sanitizer was most effective compared to those containing $62 \%$ ethanol, $70 \%$ isopropanol or $91 \%$ isopropanol, with the ethanol-based disinfectants more effective than propanol. After the 30 second contact time, the $\log _{10}$ virus reduction factors for the $99.5 \%$ ethanol, $62 \%$ ethanol-based instant hand sanitizer, $91 \%$ isopropanol and $70 \%$ isopropanol disinfectants were $1.0,0.50,0$ and 0.67 , respectively, whereas for the 2 minute contact time, the $\log _{10}$ virus reduction factors were $1.3,0.55,0.43$ and 0.55 , respectively. The virucidal efficacy of the non-alcohol-based antiseptic, povidone-iodine at $10 \%$ (equivalent to $1 \%$ available iodine), was much higher than alcohol-based hand sanitizers; hence the iodine-based compounds were considered to be more effective against FCV. The $\log _{10}$ virus reductions for the three nonalcohol-based sanitizers, $10 \%$ povidone-iodine, $3 \%$ hydrogen peroxide and $0.13 \%$ benzalkonium chloride, after 30 second contact time were 2.67, 0.09 and 0, respectively, whereas for the 2 minute contact time, they were $2.39,0.47$ and 0.22 , respectively. The virucidal efficacy of the two triclosan-based hand soaps was poor; when compared to that of rinsing hands with tap water without soap, both results were similar. The two triclosan-based hand soaps at concentrations of 0.60 and $0.115 \%$ had $\log _{10}$ virus reduction factors of 0.25 and 0.42 after 30 seconds, and 0.50 and 0.17 after 2 minute contact times, respectively, whereas simple hand 
washing without soap produced $\log _{10}$ virus reduction factors of 0.33 and 0.42 after 30 second and 2 minute contact times, respectively. Virus reduction by antimicrobial soaps may be due to mechanical removal during hand washing and not necessarily because of antimicrobial activity from the soap (Lages et al., 2008).

Whitehead \& McCue (2010) exposed disinfectants including an alcohol, acid, QUAT and phenol on an inanimate surface containing dried FCV suspension for 1 minute contact time. Sodium hypochlorite (1000 ppm), both organic acid (citric acid at $2.5 \%$ ) and inorganic acid (hydrochloric acid at $0.38 \%$ ) at $\mathrm{pH} 2.0$, and alkali (monoethanolamine) at $\mathrm{pH} 12.0$ produced a $>3 \log _{10}$ reduction and were effective in inactivating FCV. However, alcohol (ethanol and isopropanol at $60 \%$ concentration) produced 1.3 and $<0.5 \log _{10}$ reductions, respectively, while the QUAT alkyl dimethyl benzyl ammonium chloride $(3000 \mathrm{ppm})$ produced $1.17 \log _{10}$ reduction, and phenol $(550 \mathrm{ppm})$ produced a $2.0 \log _{10}$ reduction, and were not effective in inactivating the virus (Whitehead \& McCue, 2010). Whitehead \& McCue (2010) suggested that the pH of the disinfectant may have a significant role in the inactivation of FCV, with disinfectants at $\mathrm{pH} 12.0$ and $<3.0$ showing inactivation of the virus.

Hudson et al. (2007) studied norovirus inactivation by ozone gas exposure. Ozone gas levels of $25 \mathrm{ppm}$ at a high relative humidity of more than $70 \%$ resulted in a $99.9 \%$ inactivation of viruses. Ozone gas concentrations between 20 to 25 ppm, maintained for 20 minutes, decreased the concentration of norovirus (from 3 different stool samples) and FCV by a factor of more than $10^{3}$ and in some cases, beyond detection after less than an hour of exposure. Samples with virus which were dried onto hard surfaces such as plastic, steel and glass in addition to soft surfaces 
such as fabric, cotton and carpet all showed the virus to be vulnerable to ozone gas when measured by quantitative RT-PCR assay. Norovirus can be inactivated by exposure to ozone gas using a portable commercial generator in hotel room settings, cruise ship cabins and healthcare facilities (Hudson et al, 2007).

Malik et al. (2006) investigated the virucidal efficacy of different concentrations (10 to $100 \%$ ) of ethanol and isopropanol against FCV that had been dried on stainless steel surfaces for 1, 3 and 10 minutes, and the virus titer was calculated using TCID $_{50}$ assay. Ethanol $(90 \%)$ produced the highest reduction of FCV with $99.49 \%$ killed in 10 minutes and $99.35 \%$ killed in 1 minute, while $70 \%$ ethanol killed $99.19 \%$ of FCV in 1 minute, whereas $100 \%$ ethanol was less effective with $98 \%$ of the virus killed after 10 minutes. Isopropanol at 40 to $60 \%$ concentration killed $99 \%$ of FCV after 1 minute, and isopropanol at 70 to $90 \%$ concentration killed $>99 \%$ in 3 to 10 minutes, whereas $100 \%$ isopropanol was less effective with $<98 \%$ of the virus killed. Viral inactivation was most effective at $60 \%$ isopropanol after 1 minute with $99.84 \%$ virus killed, and $90 \%$ ethanol after 10 minutes with $99.49 \%$ killed (Malik et al., 2006).

Poschetto et al. (2007) studied the abilities of four disinfectants (an organic acid, aldehyde, sodium hypochlorite and hydrogen peroxide) to inactivate FCV under field conditions using human fecal material as the protein load. The presence of the organic load reduced the efficacy of the disinfectants; however, the organic acid, sodium hypochlorite and peroxide were effective against FCV when measured using $50 \%$ TCID $_{50}$ and RT-PCR, and resulted in $\geq 99.9 \%$ of virus inactivated. The organic acid containing $5 \%$ formic acid, $1 \%$ hydrogen peroxide and at least $2 \%$ glutaraldehyde at a 1 hour contact time, and $1 \%$ sodium hypochlorite (6000 ppm free 
chlorine) at a 15 minute contact time, inactivated the virus with $\geq 3 \log _{10}$, and may be used for disinfection during norovirus outbreaks (Poschetto et al., 2007).

MNV-1 was more stable than FCV at room temperature, $\mathrm{pH}$ extremes and more persistent on surfaces in wet and dry conditions. The MNV-1 capsid structure, genomic organization and replication cycle are similar to human norovirus. Because MNV-1 is more acid tolerant at surviving gastric pH levels, Cannon et al. (2006) stated that it is a more suitable surrogate virus for human norovirus studies than FCV. Norovirus stability and inactivation in the environment was compared using MNV-1 and FCV as surrogate viruses for human norovirus by evaluating the stability of both viruses after exposure to $\mathrm{pH}$ extremes, exposure to organic solvents, thermal inactivation and surface persistence under wet and dry conditions. MNV-1 was stable across the entire $\mathrm{pH}$ range of 2 to 10 , with less than $1 \log$ reduction in infectivity at $\mathrm{pH} 2$, whereas FCV was inactivated at $\mathrm{pH}$ values less than 3 and above 9 . FCV was more stable than MNV-1 at $56{ }^{\circ} \mathrm{C}$ (a 1-log reduction was reached at 3.5 and 6.7 minutes, respectively) but both viruses were inactivated by $1-\log$ at $63{ }^{\circ} \mathrm{C}$ in about 25 seconds, and in less than 10 seconds at $72{ }^{\circ} \mathrm{C}$. In addition, long-term persistence of both viruses was tested by suspending them into a fecal matrix, inoculating it onto stainless steel coupons, and holding them for up to 7 days in a controlled environment. Exposure to organic solvents such as chloroform, Freon and Vertrel showed that both surrogate viruses, MNV-1 and FCV, are relatively resistant to all three organic solvents however the recovery of MNV-1 was better than FCV. Stability of both viruses was similar at $4{ }^{\circ} \mathrm{C}$ but at room temperature in suspension, MNV-1 was more stable than FCV (Cannon et al., 2006). 
Seo et al. (2012) studied the resistance of MNV-1 to temperature, salt and $\mathrm{pH}$ using a plaque assay. MNV-1 was rapidly inactivated at temperatures above $60{ }^{\circ} \mathrm{C}$ with a $4 \log _{10}$ reduction after 10 minutes, $>3 \log _{10}$ reduction after 2 minutes at $70{ }^{\circ} \mathrm{C}$ and was entirely inactivated after 1 minute at $85{ }^{\circ} \mathrm{C} . \mathrm{MNV}-1$ also survived low salt conditions $(0.3 \% \mathrm{NaCl})$ better than high salt conditions at $(3.3-6.3 \% \mathrm{NaCl})$ at $24{ }^{\circ} \mathrm{C}$, and was relatively resistant to strong acidic conditions at $\mathrm{pH} 2$ but tolerated conditions between $\mathrm{pH} 4$ and 7 . Temperature has a greater effect on MNV-1 infectivity than salt or low pH. RT-PCR and long-template RT-PCR assay was used alongside the plaque assay, with long-template RT-PCR requiring a larger fragment of the viral genome to be intact for amplification than RT-PCR. Both RT-PCR and long-template RT-PCR assay did not reflect the inactivation of MNV-1, and significantly underestimated the inactivation of the virus by temperature, salt and $\mathrm{pH}$. A few minutes' exposure to temperatures above $70{ }^{\circ} \mathrm{C}$ proved to be most effective in inactivating MNV-1 and may be important in the prevention of norovirus outbreaks (Seo et al., 2012).

Studies have shown that sodium hypochlorite at 1000 ppm available chlorine completely inactivated FCV with a $\log _{10}$ reduction of 4.17 at 1 minute under dry condition, whereas MNV-1 was inactivated at $2600 \mathrm{ppm}$ with $>4 \log$ reduction after $0.5,1$ and 3 minute contact times (Belliot et al., 2008; Whitehead \& McCue, 2010). Another study compared human norovirus and MNV-1 using RT-PCR and plaque assay, and showed MNV-1 to be more sensitive than human norovirus to disinfectants by 1-2 log units. Quaternary ammonium-based disinfectants were ineffective with $<1$ log reduction after 10 minutes (Girard et al., 2010). In exposing MNV-1 to chemical biocides such as alcohol on stainless steel surfaces without mechanical 
action, 1-propanol was most effective in reducing MNV-1 in 5 minutes at $30 \%$ concentration with $\geq 4 \log$ reduction (Magulski et al., 2009).

Belliot et al. (2008) evaluated various disinfectants including alcohol, alcohol hand sanitizers, QUAT, sodium hypochlorite and povidone iodine-based disinfectant on MNV-1 for $0.5,1$ and 3 minute contact times, and determined the resistance of the MNV RNA genome using RT-PCR method. Ethanol and isopropanol at $60 \%$ concentration produced $>4 \log _{10}$ reduction in viral titer after a 1 minute contact time. The loss of infectivity and the continued presence of genomic RNA suggested that protein alteration was the mechanism behind the inactivation of MNV-1. Sodium hypochlorite (at $2600 \mathrm{ppm}$ available chlorine) and $1 \%$ povidone iodone produced $>4$ $\log _{10}$ reduction in infectious viral titer after 0.5 minutes of exposure; however, viral RNA was detected after povidone iodine treatment but not after chlorine treatment. The $0.25 \%$ QUAT was ineffective and produced a $1 \log _{10}$ reduction after a 1 minute contact time. Alcohol-based hand sanitizers were able to produce at least a $4 \log _{10}$ reduction in MNV titer after 1 minute exposure, and real-time RT-PCR results suggested that the alteration of the viral capsid was the mechanism which led to the inactivation of MNV-1 and that the genomic RNA was still present after the assay. Belliot et al. (2008) suggested that the MNV capsid may have been denatured by exposure to chlorine. Detection of viral RNA from real-time RT-PCR could not be related to the presence of infectious virus during plaque assay (Belliot et al., 2008).

Girard et al. (2010) studied the impact of pH, humidity and the efficacy of household disinfectants on the attachment of norovirus and MNV-1 to fomites using plaque assay and RTPCR. Human norovirus and MNV-1 attached to stainless steel after 10 minutes at $25 \%$ relative 
humidity, and although human norovirus attachment was not affected by $\mathrm{pH}, \mathrm{MNV}-1$ attached to stainless steel to a lesser extent at pH 9. Girard et al. (2010) suggested that non-enveloped viruses such as norovirus appear to survive better at high relative humidity (of $>80 \%$ ). Sodium hypochlorite at $3 \%$ concentration (30000 ppm chlorine) inactivated MNV-1 after a 5 and 10 minutes with $>4 \log _{10}$ reduction using plaque assay as opposed to a $4 \log _{10}$ reduction after 10 minutes with RT-PCR, whereas QUATs ( $N$-alkyl dimethyl benzyl ammonium chloride at 0.02 and $0.08 \%$ concentration) showed a $1 \log _{10}$ reduction after 10 minutes and $<0.5 \log _{10}$ reduction after 5 minutes with either plaque assay or TaqMan RT-PCR assay. Human norovirus was inactivated with $3 \%$ sodium hypochlorite after 10 minutes with $>3 \log _{10}$ reduction but a $2 \log _{10}$ reduction occurred after 5 minutes; however, no log reduction was observed with ethoxylated alcohols or QUATS using RT-PCR assay (Girard et al., 2010).

Magulski et al. (2009) tested the efficacy of different concentrations of alcohol, peracetic acid and glutaraldehyde on MNV-1 vacuum-dried on stainless steel discs for 5 minutes, and determined viral titer post-exposure by endpoint titration method using RAW 264.7 macrophage mouse cells. The results showed that $50 \%$ ethanol, $30 \%$ 1-propanol, peracetic acid at $1000 \mathrm{ppm}$ and glutaraldehyde at $2500 \mathrm{ppm}$ inactivated MNV-1 on stainless steel surfaces with $\geq 4 \log _{10}$ reduction after a 5 minute contact time. In particular, $40 \%$ 1-propanol was most effective and inactivated the virus by an average of $6.04 \log _{10}$ reduction, whereas 2-propanol was not able to adequately inactivate $\mathrm{MNV}-1$ since there was only $3.02 \log _{10}$ reduction after 5 minutes (Magulski et al., 2009). 


\subsection{Research Question and Hypothesis}

This study focused on determining the efficacy of common types of disinfectants used in health care facilities in the province of British Columbia, Canada. The central research question was to determine what disinfectant (at what concentration and contact time) worked optimally to inactivate norovirus on surfaces under wet and dry conditions, with and without a soil load. A soil load mixture was used to simulate an organic matrix because viruses are often present in an organic soil load. Wet conditions simulated fresh vomitus or liquid stool where viral particles were in suspension and dry conditions simulated viral particles (in vomitus or liquid stool) which underwent environmental desiccation on a surface. The latter dry condition was predicted to be more inherently challenging for the disinfectants to inactivate viral particles as this represented a two dimensional surface. In solution (wet condition), the disinfectant and viral particles were in a three dimensional status and should allow the disinfectant to perform optimally by more easily reaching the virus. Wet and dry load conditions with a soil load were also predicted to be more challenging than without a soil load because protein from the soil load may reduce disinfectant effectiveness. Five disinfectants were tested to determine the efficacy against human norovirus surrogates such as murine norovirus and feline calicivirus. The disinfectants were chosen to represent a chemical category and included bleach (sodium hypochlorite), quaternary ammonium compounds, and accelerated hydrogen peroxides. This study also compared the differences between two surrogate viruses (MNV-1 and FCV) when the selected disinfectants/cleaning agents were used. 
The hypotheses:

1. There will be no difference between wet ( 0 hour) and dry (60-90 minutes) load conditions for test carriers contaminated with the respective surrogate virus and exposed to different disinfectants/cleaning agents and concentrations, and control carriers at the 1, 5 and 10 minute contact times from the Quantitative Carrier Test-2 (QCT-2) method for MNV-1.

2. There will be no difference between wet ( 0 hour) and dry (60-90 minutes) load conditions for test carriers contaminated with the respective surrogate virus and exposed to different disinfectants/cleaning agents and concentrations, and control carriers at the 1, 5 and 10 minute contact times from the QCT-2 method for FCV.

3. There will be no difference between wet ( 0 hour) and dry (60-90 minutes) load conditions $\underline{\text { with }}$ soil load for test carriers contaminated with the respective surrogate virus and exposed to different disinfectants/cleaning agents and concentrations, and control carriers at the 1, 5 and 10 minute contact times from the QCT-2 method for MNV-1.

4. There will be no difference between wet ( 0 hour) and dry (60-90 minutes) load conditions with soil load for test carriers contaminated with the respective surrogate virus and exposed to different disinfectants/cleaning agents and concentrations, and control carriers at the 1, 5 and 10 minute contact times from the QCT-2 method for FCV.

5. There will be no difference between wet ( 0 hour) and dry (60-90 minutes) load conditions, with and without soil load, for test carriers contaminated with the respective surrogate virus and exposed to different disinfectants/cleaning agents and concentrations, and control carriers at the 1,5 and 10 minute contact times from the QCT-2 method for MNV-1. 
6. There will be no difference between wet (0 hour) and dry (60-90 minutes) load conditions, with and without soil load, for test carriers contaminated with the respective surrogate virus and exposed to different disinfectants/cleaning agents and concentrations, and control carriers at the 1,5 and 10 minute contact times from the QCT-2 method for FCV. 
Table 1. Comparison of temperature, environmental and pH stability of norovirus, hepatitis A virus, Staphylococcus aureus and Escherichia coli O157:H7.

\begin{tabular}{|c|c|c|c|c|c|c|c|}
\hline \multirow{2}{*}{ Microorganism } & \multicolumn{2}{|c|}{ Temperature survival } & \multicolumn{3}{|c|}{ Environmental survival } & \multirow[t]{2}{*}{$\mathrm{pH}$ survival } & \multirow[t]{2}{*}{ Reference } \\
\hline & Refrigeration & Heat & Surface & Stainless steel & Hands & & \\
\hline \multicolumn{8}{|l|}{ Norovirus } \\
\hline Human Norovirus & Yes $^{1}$ & $\begin{array}{l}\text { Up to } 60^{\circ} \mathrm{C}^{1}, 76.6^{\circ} \mathrm{C} \text { for } \\
\text { capsid }^{2}\end{array}$ & $\begin{array}{l}\text { At least } 10 \text { days }{ }^{1} \text {, } \\
\text { can last for } 21-28 \\
\text { days }^{4}\end{array}$ & & $\begin{array}{l}\text { Unchanged after } \\
120 \min ^{4}\end{array}$ & & $1,2,4$ \\
\hline Murine norovirus & $4{ }^{\circ} \mathrm{C}$ in juice ${ }^{3}$ & $\begin{array}{l}\text { Stable at } 37^{\circ} \mathrm{C}^{5} \text {, inactivated } \\
\text { at } 63{ }^{\circ} \mathrm{C}^{6}\end{array}$ & $\begin{array}{l}\text { Stable for } 40 \\
\text { days at dried } \\
\text { state }^{19}\end{array}$ & $\begin{array}{l}\text { Stable in } 4{ }^{\circ} \mathrm{C} \\
\text { and room } \\
\text { temperature in } \\
\text { fecal matrix }\end{array}$ & & $2-10^{6}$ & $3,5,6,19$ \\
\hline Feline calicivirus & $4{ }^{\circ} \mathrm{C}$ in juice ${ }^{3}$ & $\begin{array}{l}\text { Stable at } 37{ }^{\circ} \mathrm{C}^{5} \text {, inactivated } \\
\text { at } 63{ }^{\circ} \mathrm{C}^{6}, 63.3^{\circ} \mathrm{C} \text { for } \\
\text { capsid }\end{array}$ & $\begin{array}{l}21-28 \text { days dried, } \\
\text { room } \\
\text { temperature }^{1}\end{array}$ & $\begin{array}{l}\text { Present at } 4{ }^{\circ} \mathrm{C} \\
\text { in fecal matrix }\end{array}$ & & $3-9^{6}$ & $1,3,5,6$ \\
\hline Snow Mountain virus & & & 42 days $^{4}$ & & $\begin{array}{l}\text { Unchanged after } \\
120 \min ^{4}\end{array}$ & & 4 \\
\hline Hepatitis A virus & $\begin{array}{l}\text { Yes and } \\
\text { freezing }{ }^{17}\end{array}$ & $\begin{array}{l}\text { Stable at } 37{ }^{\circ} \mathrm{C}^{5} \text {, inactivated } \\
\text { at } 85-90{ }^{\circ} \mathrm{C}^{17}\end{array}$ & $\begin{array}{l}\text { Several days } \\
\text { indoors }{ }^{17}\end{array}$ & $\begin{array}{l}\text { Several days in } \\
\text { fecal matter }{ }^{18}\end{array}$ & $\begin{array}{l}\text { Can survive for } \\
\text { several hours } \\
{ }^{18} \text {, } \\
9.2 \% \text { transfer of } \\
\text { virus from } \\
\text { fingerpad to } \\
\text { food }^{16}\end{array}$ & & $\begin{array}{l}5,16,17 \\
18\end{array}$ \\
\hline Staphylococcus aureus & & $\begin{array}{l}7-48.5^{\circ} \mathrm{C} \text { range, optimum } \\
30-37^{\circ} \mathrm{C}^{9}\end{array}$ & $\begin{array}{l}\text { At least } 1 \text { day on } \\
\text { all surfaces, } 22 \\
\text { to }>90 \text { days on } \\
\text { plastics }{ }^{11}\end{array}$ & At least $72 \mathrm{~h}^{8}$ & & $\begin{array}{l}4.0-10.0 \\
\text { range, } \\
\text { optimum } 6.0- \\
7.0^{10}\end{array}$ & $8,9,10,11$ \\
\hline E. coli $\mathrm{O} 157: \mathrm{H} 7$ & $\begin{array}{l}-20{ }^{\circ} \mathrm{C} \text { in } \\
\text { frozen foods } 15\end{array}$ & $\begin{array}{l}\text { Broad range, growth } \\
\text { between } 10-45^{\circ} \mathrm{C}^{15}, \\
\text { optimum growth at } 37^{\circ} \mathrm{C}^{7}\end{array}$ & $\begin{array}{l}\text { Days to almost a } \\
\text { year }^{7}\end{array}$ & $\begin{array}{l}\text { Ability to attach } \\
\text { and colonize }\end{array}$ & & $\begin{array}{l}\text { Can go low } \\
\text { as } \sim 2.5^{7} \text {, } \\
\text { survive } \\
\geq 4.0^{12} \text { to } \\
9.0^{13}\end{array}$ & $\begin{array}{l}7,12,13 \\
14,15\end{array}$ \\
\hline
\end{tabular}

Sources: ${ }^{1}$ Weber et al., 2010; ${ }^{2}$ Topping et al., 2009; ${ }^{3}$ Horm \& D'Souza, 2011; ${ }^{4}$ Liu et al., 2009; ${ }^{5}$ Gibson \& Schwab, 2011; ${ }^{6}$ Cannon et al., 2006; ${ }^{7}$ Van Elsas et al., 2011; ${ }^{8}$ Fuster-Valls et al., 2008; ${ }^{9}$ Schmitt et al., 1990; ${ }^{10}$ Bergdoll, $1989 ;{ }^{11}$ Neely \& Maley, 2000; ${ }^{12}$ Conner \& Kotrola, 1995; ${ }^{13}$ Yuk \& Marshall, $2004 ;{ }^{14}$ Dourou et al., 2011; ${ }^{15}$ Duffy, 2006; ${ }^{16}$ Bidawid et al., 2000; ${ }^{17}$ Sattar et al., 2000, ${ }^{18}$ Mbithi et al., 1991; ${ }^{19}$ Magulski et al., 2009. 
Table 2. Primers and probes used in real-time quantitative RT-PCR for detecting norovirus genogroups I and II.

\begin{tabular}{|l|l|l|}
\hline Genogroup & Primer or probe & Sequence $\left(5^{\prime} \rightarrow\right.$ 3') \\
\hline I & Primer COG1F & CGYTGGATGCGNTTYCATGA \\
& Primer COG1R & CTTAGACGCCATCATCATTYAC \\
& Probe RING1(a)-TP & FAM-AGATYGCGATCYCCTGTCCA-TAMRA ${ }^{\text {a }}$ \\
& Probe RING1(b)-TP & FAM-AGATCGCGGTCTCCTGTCCA-TAMRA \\
& Primer 431 & TGGACIAGRGGICCYAAYCA \\
& Primer 432 & TGGACICGYGGICCYAAYCA \\
& Primer 433 & GAAYCTCATCCAYCTGAACAT \\
& Primer 434 & GAASCGCATCCARCGGAACAT \\
\hline II & Primer COG2F & CARGARBCNATGTTYAGRTGGATGAG \\
& Primer COG2R & TCGACGCCATCTTCATTCACA \\
& Probe RING2-TP & FAM-TGGGAGGGCGATCGCAATCT-TAMRA \\
& Primer 431 & TGGACIAGRGGICCYAAYCA \\
& Primer 432 & TGGACICGYGGICCYAAYCA \\
& Primer 433 & GAAYCACATCCAYCTGAACAT \\
& Primer 434 & GAASCGCATCCARCGGAACAT \\
\hline
\end{tabular}

${ }^{a} 6$-Carboxyfluorescein (FAM) is reporter dye coupled in 5' end of oligonucleotide, and 6-carboxy-tetramethylrhodamine (TAMRA) is quencher dye coupled in 3' end of oligonucleotide.

Sources: Health Canada, 2010; Kageyama et al., 2003. 
Table 3. Summary of effectiveness of commonly used disinfectants, $\mathrm{pH}$ and temperature on MNV-1 and FCV surrogates of human norovirus.

\begin{tabular}{|c|c|c|}
\hline \multirow[t]{2}{*}{ Disinfectants and other conditions } & \multicolumn{2}{|c|}{ Inactivation of surrogate virus } \\
\hline & MNV-1 & FCV \\
\hline \multicolumn{3}{|l|}{ Disinfectants } \\
\hline Sodium hypochlorite & ${ }^{1} 2600$ ppm, 0.5 minutes, $>4 \log _{10}$ reduction ${ }^{c}$ & ${ }^{11} 1000$ ppm, 1 minute, $>3 \log _{10}$ reduction $^{a}$ \\
\hline Accelerated hydrogen peroxide & NA & ${ }^{8} 5000 \mathrm{ppm}, 1$ minute, $>6.25 \log _{10}$ reduction \\
\hline Hydrogen peroxide & NA & $\begin{array}{l}{ }_{5}^{5} \%, 2 \text { minutes, } 0.47 \log _{10} \text { reduction }^{\mathrm{a}} \\
{ }^{9} 1 \%, 60 \text { minutes, } \geq 3 \log _{10} \text { reduction }^{\mathrm{c}}\end{array}$ \\
\hline $\begin{array}{l}\text { Alcohol hand sanitizer } \\
\text { Ethanol }\end{array}$ & NA & ${ }^{5} 62 \%, 2$ minutes, $0.55 \log _{10}$ reduction $^{a}$ \\
\hline $\begin{array}{l}\text { Alcohol } \\
\text { Ethanol } \\
\text { 1-propanol } \\
\text { 2-propanol }\end{array}$ & $\begin{array}{l}{ }^{1} 60 \%, 1 \text { minute, }>4 \log _{10} \text { reduction }^{c} \\
{ }^{6} 30 \%, 5 \text { minutes, } \geq 4 \log _{10} \text { reduction }^{a} \\
{ }^{6} 40 \%, 5 \text { minutes, } 6.04 \log _{10} \text { reduction }^{a} \\
{ }^{1} 60 \%, 1 \text { minute, }>4 \log _{10} \text { reduction }^{c}\end{array}$ & 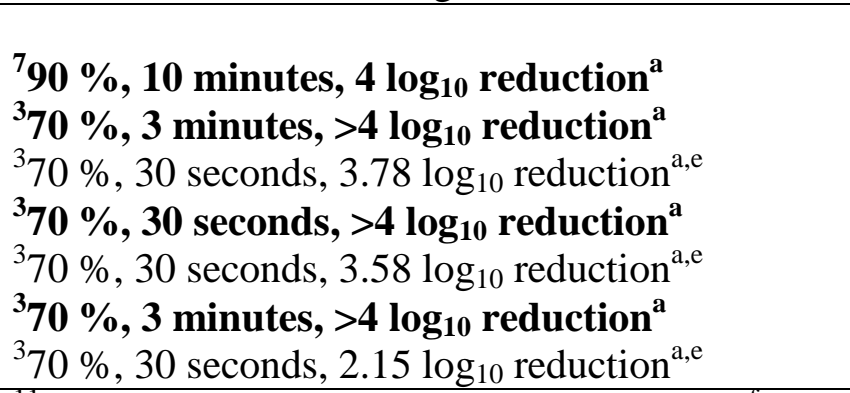 \\
\hline Quaternary ammonium compound & ${ }^{1} 0.25 \%, 1$ minute, $1 \log _{10}$ reduction $^{\mathrm{c}}$ & ${ }^{11} 3000 \mathrm{ppm}, 1$ minute, $1.17 \log _{10}$ reduction $^{\mathrm{a}, \mathrm{f}}$ \\
\hline Phenol & NA & ${ }^{11} 550 \mathrm{ppm}, 1$ minute, $2 \log _{10}$ reduction $^{\mathrm{a}}$ \\
\hline Iodine & ${ }^{1} 1 \%, 0.5$ minutes, $>4 \log _{10}$ reduction ${ }^{c}$ & ${ }^{5} 1 \%, 30$ seconds, $2.67 \log _{10}$ reduction $^{a}$ \\
\hline Ozone & NA & ${ }^{4} 20-25 \mathrm{ppm}$ (gas), 20 minutes, $>3 \log _{10}$ reduction $^{\mathrm{c}}$ \\
\hline Antibacterial liquid soap $^{\mathrm{d}}$ & NA & ${ }^{5} 0.60 \%, 2$ minutes, $0.50 \log _{10}$ reduction $^{\mathrm{a}}$ \\
\hline $\mathrm{pH}$ & ${ }^{2}$ Resistant between $2-10^{\mathrm{b}}$ & ${ }^{2}$ Inactivated $<3$ and $>9^{b}$ \\
\hline Temperature & $\begin{array}{l}{ }^{2} 63{ }^{\circ} \mathrm{C}, 25 \text { seconds } \\
{ }^{b} 72{ }^{\circ} \mathrm{C},<10 \text { seconds } \\
{ }^{10}>60 \\
{ }^{\circ} \mathrm{C}, 10 \text { minutes, } 4 \log _{10} \text { reduction }^{\mathrm{b}} \\
{ }^{10} 70^{\circ} \mathrm{C}, 2 \text { minutes, }>3 \log _{10} \text { reduction } \\
{ }^{10} 85{ }^{\circ} \mathrm{C}, 1 \text { minute }\end{array}$ & $\begin{array}{l}{ }^{2} 63{ }^{\circ} \mathrm{C}, 25 \text { seconds }{ }^{b} \\
{ }^{2} 72{ }^{\circ} \mathrm{C},<10 \text { seconds }{ }^{b}\end{array}$ \\
\hline
\end{tabular}

Bolded values indicate inactivation of the surrogate virus. NA refers to not applicable.

${ }^{a}$ Cytopathic effects observed using end-point titration method (50\% tissue culture infectious dose $\left.\left(\mathrm{TCID}_{50}\right)\right)$. ${ }^{\mathrm{b}}$ Plaque assay. ${ }^{\mathrm{c}}$ Reverse transcription polymerase chain reaction (RT-PCR). ${ }^{\mathrm{d}}$ Triclosan-based antimicrobial liquid soap. ${ }^{\mathrm{e}}$ Results based on contaminated fingertips. ${ }^{\mathrm{f}}$ Alkyl dimethyl benzyl ammonium chloride. Sources: ${ }^{1}$ Belliot et al., 2008; ${ }^{2}$ Cannon et al., 2006; ${ }^{3}$ Gehrke et al., 2004; ${ }^{4}$ Hudson et al., 2007; ${ }^{5}$ Lages et al., 2008; ${ }^{6}$ Magulski et al., $2009 ;{ }^{7}$ Malik et al., 2006 ;

${ }^{8}$ Omidbakhsh \& Sattar, 2006; ${ }^{9}$ Poschetto et al., 2007; ${ }^{10}$ Seo et al., 2012; ${ }^{11}$ Whitehead \& McCue, 2010. 


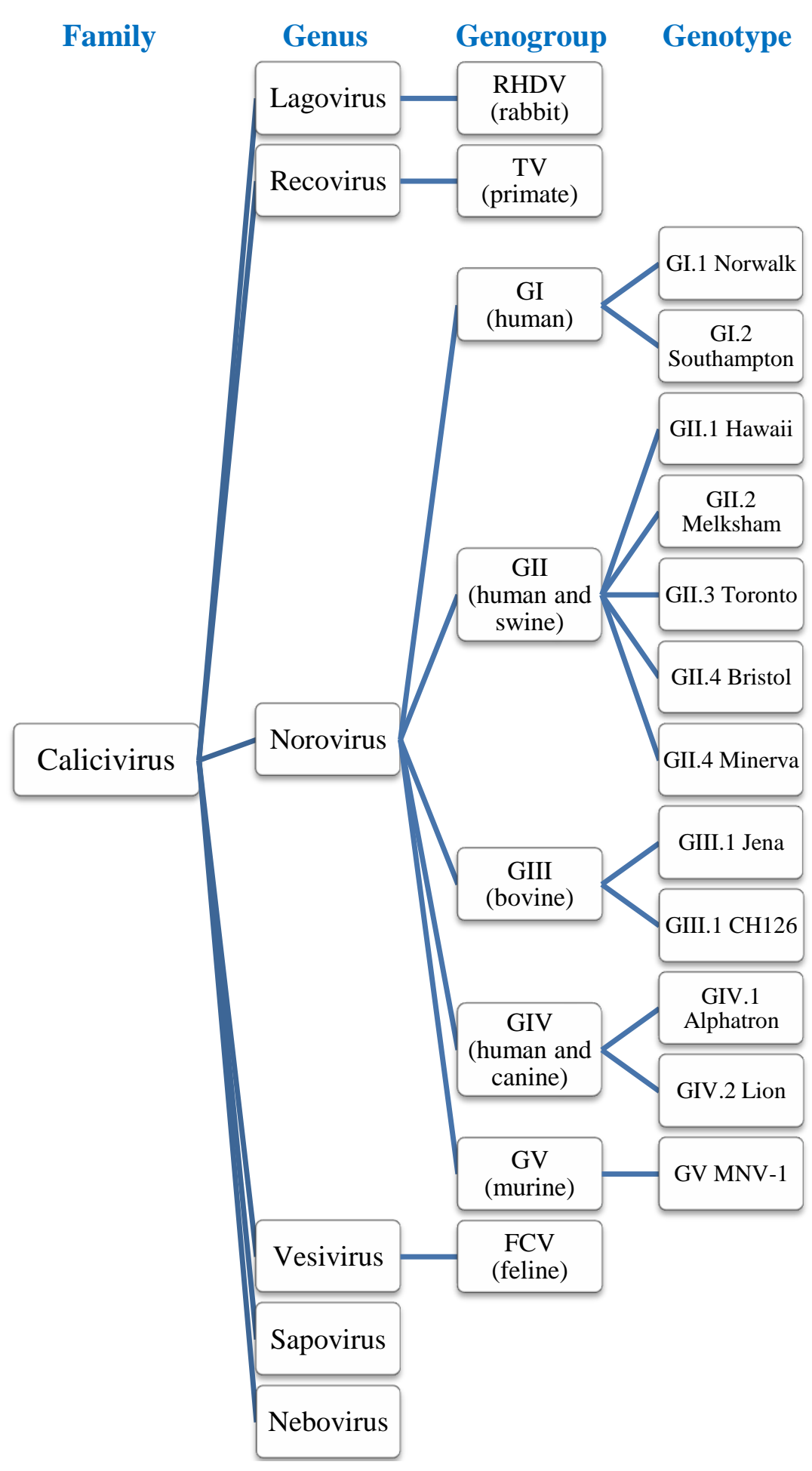

Figure 1. Diagram of Calicivirus family, genus, genogroup and genotype.

RHDV refers to rabbit haemorrhagic disease virus. TV refers to Tulane virus. MNV-1 refers to murine norovirus. FCV refers to feline calicivirus.

Sources: Karst, 2010; Patel et al., 2009. Adapted from Karst, 2010. 


\section{Genomic RNA}

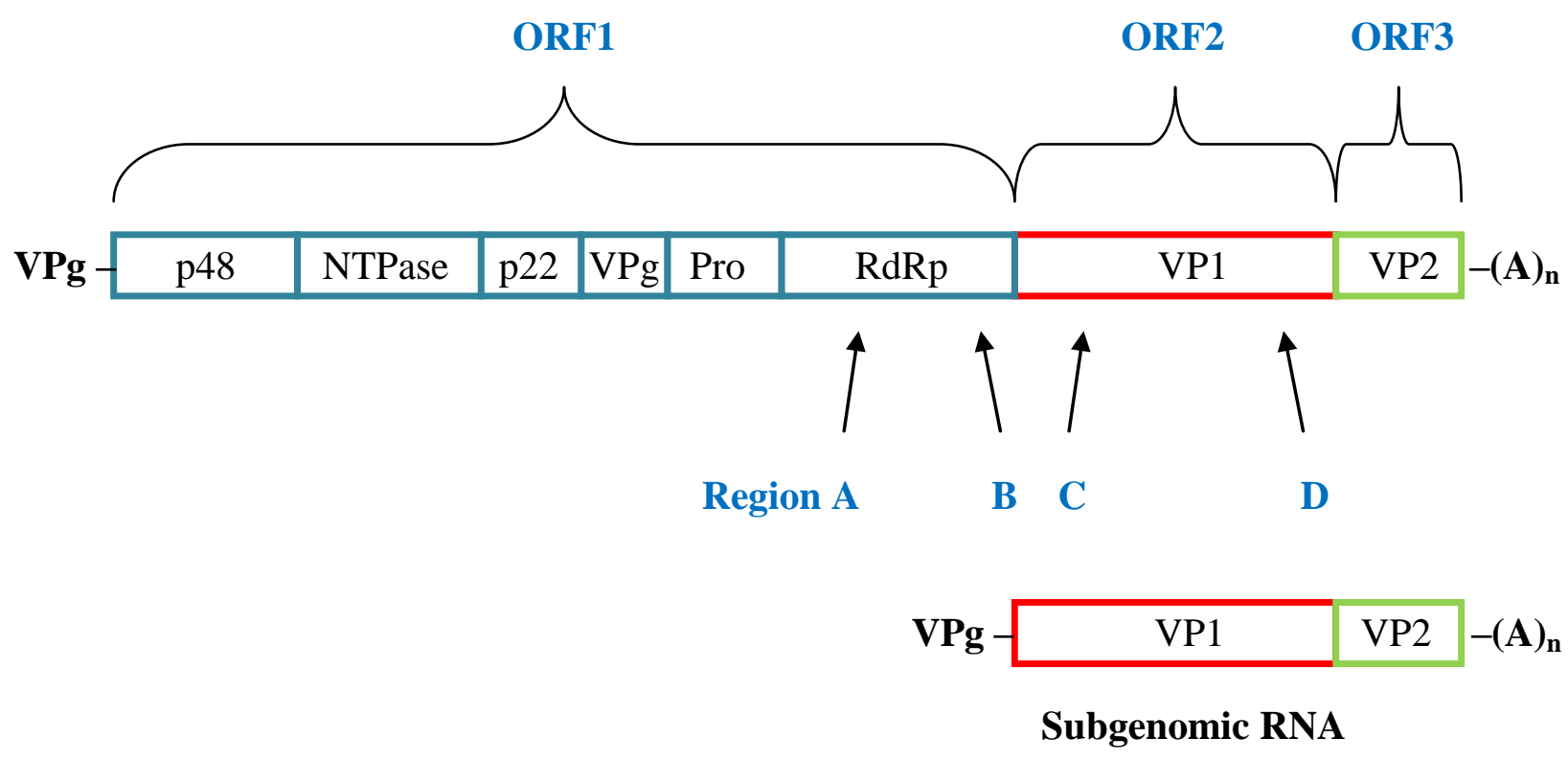

Figure 2. Norovirus genome with protein products and positions of regions A-D used for detection and genotyping.

The genome consists of three open reading frames (ORF). ORF1 encodes a polyprotein of 7 nonstructural protein products, ORF2 encodes a major structural capsid protein (VP1), and ORF3 encodes a minor structural protein (VP2). The VPg-linked subgenomic RNA which encodes VP1 and VP2 is located below the ORFs. The subgenomic RNA is covalently linked to the VPg at the 5 ' end and polyadenylated at the 3 ' end.

Sources: Hardy, 2005; Karst, 2010; Vinjé et al., 2004. Adapted from Karst, 2010. 


\section{CHAPTER 2 Materials and Methodology}

This study tested the efficacy of five common types of disinfectants to determine which environmental conditions (wet or dry load conditions), disinfectant concentrations and contact times resulted in the inactivation of MNV-1 and FCV, in the presence and absence of a soil load (Figure 3).

The quantitative carrier test-2 (QCT-2) method to assess the efficacy of disinfectants and/or cleaning agents on surrogate viruses followed the set of procedures reported by Sattar et al. (2003). Methods for virus stock preparation, tissue cell culture propagation and plaque assay followed the set of procedures reported by Wobus et al. (2004) and T. Lynn (personal communication, October 15, 2007), with a few minor changes such as the viral plaque incubation period.

The thawing, passaging, freezing, harvesting and virus propagation procedures for frozen cell cultures and viruses were followed using the protocols set by the American Type Culture Collection (2012a; 2012b; 2012c), Bidawid et al. (2003), Grist et al. (1979), Schmidt \& Emmons (1989), and Wobus et al. (2004).

\subsection{Surrogate Viruses and Cell Cultures}

MNV-1 was obtained as a gift from Dr. Herbert W. (Skip) Virgin IV of the Washington University School of Medicine, Department of Pathology and Immunology (St. Louis, MO). FCV strain F9 (ATCC VR-782) was purchased from the American Type Culture Collection (ATCC) (Manassas, VA). The viruses were stored in a $-80^{\circ} \mathrm{C}$ freezer until use. The 
macrophage mouse RAW 264.7 cell cultures (ATCC TIB-71) and Crandell Reese feline kidney (CRFK) cell cultures (ATCC CCL-94) were purchased from ATCC (Manassas, VA) and stored in a liquid nitrogen storage tank until use.

\subsection{Thawing Frozen Cell Cultures}

Cryovials of CRFK and RAW 264.7 cells were taken out of the liquid nitrogen tank, immediately wrapped with a wet paper towel and partially submerged (three-quarters way) in a flask of lukewarm water at $37^{\circ} \mathrm{C}$ to thaw the cells (Grist et al., 1979; Schmidt \& Emmons, 1989). The cryovial was decontaminated by spraying and wiping with $70 \%$ isopropyl alcohol in the laminar flow hood prior to opening (Schmidt \& Emmons, 1989). Thawed cells were transferred into a sterile tube containing $10 \mathrm{~mL}$ of alpha-Minimum Essential Medium (alphaMEM) (Gibco, catalog \#12000-063) and centrifuged at $432 \times g$ or $2000 \mathrm{rpm}$ (Heraeus Clinifuge 75003539 rotor Sepatech $3760,9.652 \mathrm{~cm}$ radius) for 10 minutes at room temperature (Grist et al., 1979). The supernatant was discarded and the cell pellet gently resuspended in fresh alphaMEM before being transferred into a sterile T-25 $\left(25 \mathrm{~cm}^{2}\right)$ tissue-culture treated polystyrene cell culture flask (Corning, catalog \#430639) containing $10 \mathrm{~mL}$ of alpha-MEM and $20 \%$ FBS (Gibco, \#12483-020) (e.g., $8 \mathrm{~mL}$ of alpha-MEM and $2 \mathrm{~mL}$ of FBS), or a sterile T-75 (75 $\left.\mathrm{cm}^{2}\right)$ tissue-culture treated polystyrene cell culture flask (Corning, catalog \#430720) containing $25 \mathrm{~mL}$ of alpha-MEM and $20 \%$ FBS for propagation (Grist et al., 1979; Schmidt \& Emmons, 1989). Flasks were gently rocked to allow the suspended cells to disperse prior to incubation at $37^{\circ} \mathrm{C}$ in a $\mathrm{CO}_{2}$ incubator overnight (Grist et al., 1979; Schmidt \& Emmons, 1989). After 24 hours, the T-25 cell culture flask containing the growth medium was removed and replaced with new growth medium (10 mL of alpha-MEM and $10 \%$ FBS) (Schmidt \& Emmons, 1989). The T-25 
cell culture flask was kept at $37^{\circ} \mathrm{C}$ in the $\mathrm{CO}_{2}$ incubator and refreshed with new growth medium once every 2-3 days until a cell monolayer of 80-90 \% confluence formed (Grist et al., 1979; Schmidt \& Emmons, 1989).

\subsection{Passaging Cell Cultures}

Cells were passaged into T-150 $\left(150 \mathrm{~cm}^{2}\right)$ tissue-culture treated polystyrene cell culture flasks (BD Falcon, catalog \#355000) each containing $50 \mathrm{~mL}$ of alpha-MEM and $10 \%$ FBS. To passage RAW 264.7 cell culture into new flasks once it was at 80-90 \% confluence, the growth medium was discarded and cell scrapers (BD Falcon, catalog \#353089) were used to dislodge cells adhering to the flask surface (ATCC, 2012b; Wobus et al., 2004).

CRFK cell monolayers were disrupted with a trypsin-versene solution containing $0.3 \%$ trypsin (Difco, ref 215240) and $0.02 \%$ ethylenediaminetetraacetic acid disodium salt (EDTA) (AnalaR, B10093) (Bidawid et al., 2003; Schmidt \& Emmons, 1989). After 2 minutes, the trypsin solution was removed and the flask was allowed to rest at room temperature in the laminar flow hood for 5 minutes to allow for cell detachment (Bidawid et al., 2003; Schmidt \& Emmons, 1989). The flask was tapped on the palm of the hand to promote detachment of the cells and an appropriate volume of fresh growth medium was added to the flask (ATCC, 2012a; Schmidt \& Emmons, 1989).

A different procedure was used for passaging RAW 264.7 cell cultures. After discarding the growth medium, alpha-MEM was added and the cell monolayer was disrupted by scraping the cell monolayer with a cell scraper (ATCC, 2012b). For RAW 264.7 cells, the subcultivation 
ratio was between 1:3 and 1:6 (ATCC, 2012b). For example, if a 1:4 subcultivation ratio was used for RAW 264.7 cells, one T-75 flask would be passaged into four new T-75 flasks (or two T-150 flasks). The $4 \mathrm{~mL}$ of cells were suspended and mixed before $1 \mathrm{~mL}$ would be dispensed to each T-75 flask (or $2 \mathrm{~mL}$ of cells into each T-150 flask) containing new growth medium. Flasks were incubated at $37^{\circ} \mathrm{C}$ in a $\mathrm{CO}_{2}$ incubator and refreshed with new growth medium once every 2-3 days until the cell culture reached 80-90 \% confluence (ATCC, 2012b; Grist et al., 1979; Schmidt \& Emmons, 1989).

For CRFK cells, the subcultivation ratio was between 1:3 and 1:8 and a trypsin-versene solution was used to dislodge the cell monolayer for passaging (ATCC, 2012a; Bidawid et al., 2003; Grist et al., 1979; Schmidt \& Emmons, 1989).

\subsection{Harvesting and Preservation of Cells for Frozen Stock}

Once the cell culture reached $80-90 \%$ confluence, they could be harvested for cell culture stocks. The contents of one flask (T-75 or T-150) are required for one cryovial of frozen stock. The freezing medium included alpha-MEM growth medium with $10 \%$ FBS and $10 \%$ dimethyl sulfoxide (DMSO) (Sigma-Aldrich, catalog \#D8418-250ML) (Schmidt \& Emmons, 1989). Cells from each tissue culture flask were dislodged and transferred to a sterile falcon tube, then centrifuged at $432 \times g$ or $2000 \mathrm{rpm}$ (Heraeus Clinifuge 75003539 rotor Sepatech $3760,9.652 \mathrm{~cm}$ radius) for 10 minutes at room temperature (Grist et al., 1979). After centrifugation, old media was discarded and the cell pellet resuspended in new alpha-MEM before being transferred to a new sterile falcon tube containing FBS and DMSO (Grist et al., 1979). The suspension was gently vortexed and $1 \mathrm{~mL}$ transferred to each cryovial which was labelled prior to being placed 
in a biocell and held in a $-80^{\circ} \mathrm{C}$ freezer overnight (Grist et al., 1979; Schmidt \& Emmons, 1989). The cryovials were taken out of the biocell and transferred the next day to a liquid nitrogen tank for long-term storage (ATCC, 2012a; ATCC, 2012b; Grist et al., 1979; Schmidt \& Emmons, 1989).

\subsection{Virus Propagation for Frozen Stock}

The virus stock stored at $-80^{\circ} \mathrm{C}$ was quickly thawed and $300 \mu \mathrm{L}$ of the virus was inoculated onto a confluent cell monolayer in a T-75 flask (Bidawid et al., 2003; Grist et al., 1979; Schmidt \& Emmons, 1989). The flask was incubated for 1 hour at room temperature while being rocked every 5 minutes to allow for virus adsorption (Bidawid et al., 2003). Subsequently, $25 \mathrm{~mL}$ of alpha-MEM containing $2 \%$ FBS were added to the flask (Bidawid et al., 2003). The infected monolayer was kept in a $\mathrm{CO}_{2}$ incubator at $37{ }^{\circ} \mathrm{C}$ for between 1 and 3 days (for MNV-1), or between 16 and $24 \mathrm{~h}$ (for FCV) (Bidawid et al., 2003).

Flasks were monitored each day for virus-induced cytopathic effects consisting of cell detachment and the presence of floating or clumped cells (Bidawid et al., 2003). Once viral infection of $90 \%$ of the monolayer was manifesting a cytopathic effect, the flask was frozen and thawed (freeze-thawed) three times at $-80{ }^{\circ} \mathrm{C}$ (Bidawid et al., 2003). Contents of the flask were transferred to a sterile falcon tube and centrifuged at $3000 \mathrm{rpm}(1892 \times g)$ (Hettich Zentrifugen Rotanta 460 centrifuge, $18.8 \mathrm{~cm}$ radius) for 20 minutes to clarify the supernatant and remove cell debris. Aliquots of $1 \mathrm{~mL}$ were placed in each cryovial and labelled prior to storage in a $-80{ }^{\circ} \mathrm{C}$ freezer for storage until use (Bidawid et al., 2003). The concentration of virus in the stock 
preparation was determined by plaque assay. When necessary, the virus was passaged to obtain a higher titer or new working stock.

\subsection{Virus Inactivation Tests: Quantitative Carrier Test 2 (QCT-2) Method}

The stainless steel disc-based QCT-2 method was followed using the protocol set by Sattar et al. (2003) (Figure 4). MNV-1 and FCV were both tested in triplicate under wet and dry load conditions, with and without soil load.

Flat-bottomed glass vials (\#5260-GV) and black autoclavable caps (\#5299-G) were purchased from Galaxy Glass (P.O. Box 238, 7 Greenwood Ave., Newfield, NJ 08344;

galaxyg183@aol.com). The $1 \mathrm{~cm}$ diameter stainless steel discs were purchased from Muzeen \& Blythe Ltd. (187 Sutherland Ave., Winnipeg, Manitoba, Canada, R2W 3E6; 204-943-9519, sales@muzeenblythe.net). Stainless steel discs were custom made from sheets of AISI brushed stainless steel type 430. The glass vials, black caps and stainless steel discs were washed, soaked and scrubbed with a labware brush in a $1 \%$ solution of 7X Cleaning Solution (ICN Biomedicals Inc., Costa Mesa, CA, catalog \#76-670-94). The items were first rinsed under tap water, then with distilled, deionized water before being allowed to air-dry for 1 to 2 days. Items were inspected for any chips, cracks or damage; items with any damage were discarded. The black caps were partially screwed shut on the lids of the glass vials and put in brown paper bags, whereas the stainless steel discs were placed on a $150 \mathrm{~mm}$ diameter Whatman No. 1 filter paper (Schleicher \& Schuell, Whatman International Ltd., England, catalog \#1001150), and put in and covered with a $15 \mathrm{~cm}$ glass petri dish (both bottom and lid) (Pyrex® USA). The stainless steel 
discs, glass vials and black caps were sterilized by autoclave at $121^{\circ} \mathrm{C}$ for 30 minutes prior to use.

The cryovial containing the selected virus was taken out of a $-80{ }^{\circ} \mathrm{C}$ freezer, allowed to thaw, and was vortexed for 60 seconds before $10 \mu \mathrm{L}$ of the virus was added onto the centre of each sterile stainless steel disc carrier (Sattar et al., 2003). The virus containing the soil load contained a soil load mixture consisting of bovine mucin, tryptone and bovine serum albumin (BSA) (Sattar et al., 2003). The stainless steel discs were placed into glass vials, inoculum side up (Sattar et al., 2003). The virus titer in the suspension was determined by serial dilution through plaque assay. The inoculum was exposed to wet and dry load conditions, with the wet load not exposed to any drying, whereas the dry load was allowed to dry for between 60-90 minutes in a biosafety lab cabinet at room temperature. The test biocide at $50 \mu \mathrm{L}$ (at 1/2, 1/4, 1/10, 1/20, 1/40, 1/80, 1/160, $1 / 320,1 / 640$ and $1 / 1280$ concentrations) was added to each stainless steel disc carrier containing the virus and held for 1, 5 and 10 minute contact time before $950 \mu \mathrm{L}$ of neutralizer with Earle's Balanced Salt Solution (EBSS) (Gibco, \#14155-063) was added to arrest the activity of the disinfectant or cleaning agent (Sattar et al., 2003). Control carriers had $50 \mu \mathrm{L}$ of EBSS added instead of test biocide (Sattar et al., 2003). EBSS was selected due to its suitability as a diluent for virus recovery and for making tissue extracts or dilutions of viruses (Grist et al., 1979). The contents of each vial were vortexed for 60 seconds before the eluates were transferred into a sterile $2 \mathrm{~mL}$ vial and serial dilutions were done using alpha-MEM as a diluent (Sattar et al., 2003). 


\subsection{Plaque Assay}

The plaque assay method was followed using the protocol reported by Bidawid et al. (2003), Wobus et al. (2004) and T. Lynn (personal communication, October 15, 2007) with a few minor changes to the procedure such as the viral plaque incubation period (Figure 4).

To prepare for plaque assay, RAW 264.7 or CRFK cells were transferred to tissue culture 6-well multi-well plates (BD Falcon, catalog \#353846) (Wobus et al., 2004). Cells were detached from the flasks, transferred into sterile falcon tubes and centrifuged at $432 \times g(2000 \mathrm{rpm})$ (Heraeus Clinifuge 75003539 rotor Sepatech 3760, $9.652 \mathrm{~cm}$ radius) for 10 minutes. The medium was discarded and the cell pellet resuspended in fresh growth medium. The cell concentration was determined by a hemocytometer after staining with a $0.1 \%(\mathrm{w} / \mathrm{v})$ crystal violet solution (Fisher Scientific, \#C-581) to determine the cell count (cells/mL) (Grist et al., 1979; Schmidt \& Emmons, 1989). The cell density was adjusted to $1 \times 10^{6}$ viable cells per $1 \mathrm{~mL}$ of alpha-MEM with $10 \%$ FBS, and $2.5 \mathrm{~mL}$ of the preparation was added to each well of a 6-well plate and incubated at $37{ }^{\circ} \mathrm{C}$ in a $\mathrm{CO}_{2}$ incubator until the cell monolayer reached $\sim 90 \%$ confluence (Wobus et al., 2004). RAW 264.7 cells were incubated for 1 day whereas CRFK cells were incubated for 2 days to achieve this state (Bidawid et al., 2003; Wobus et al., 2004).

Serial ten-fold dilutions of the virus preparation were prepared and $500 \mu \mathrm{L}$ aliquots of each dilution were added to each of the three wells (e.g., in triplicate) and incubated at room temperature for 1 hour while rocking the 6-well plates gently (to disperse the virus suspension) every 10-15 minutes (Bidawid et al., 2003; Wobus et al., 2004). After incubation, the aliquots were removed from each well and $2 \mathrm{~mL}$ of agarose overlay mixture (tempered to $47^{\circ} \mathrm{C}$ ) 
containing equal volumes of $1.25 \%$ low melting point SeaPlaque agarose and $2 \mathrm{X}$ alpha-MEM with $10 \%$ FBS was slowly added to each well (Bidawid et al., 2003; Wobus et al., 2004). The agarose overlay mixture was carefully added down the side of each well at a rate of one drop per second. The agarose overlay had to be applied with care to avoid causing the cell monolayer to detach, tear apart, roll over or peel off the plastic polystyrene surface of the well (Figure C5) (Bidawid et al., 2003). The plates were allowed to sit at room temperature for 10 minutes to allow the agarose to solidify and were then incubated at $37^{\circ} \mathrm{C}$ in a $\mathrm{CO}_{2}$ incubator for $36-40$ hours (MNV-1) or 40-45 hours (FCV). After incubation, a $2 \mathrm{~mL}$ second agarose overlay mixture (tempered to $56{ }^{\circ} \mathrm{C}$ ) containing equal volumes of $1.25 \%$ SeaKem LE agarose, $2 \mathrm{X}$ alpha-MEM with $10 \%$ FBS and $0.1 \%$ neutral red solution (Sigma, catalog \#57993-100ML-F) was added to each well so viable cells would be stained by the neutral red dye (Bidawid et al., 2003; Wobus et al., 2004). The plates were held at room temperature for 10 minutes to allow the agarose to solidify and incubated again at $37^{\circ} \mathrm{C}$ in a $\mathrm{CO}_{2}$ incubator for up to 24 hours before plaques were counted (Bidawid et al., 2003; Wobus et al., 2004). For MNV-1, the plaques were viewed and counted during the 5-8 hour and 24 hour mark (Figure C3), whereas FCV plaques were viewed at the 24 hour mark (Figure C4) (Wobus et al., 2004). Plaques from test and control carriers were visualized and counted to determine the virus concentration as plaque forming units (PFU) per mL (Figure C2) (Bidawid et al., 2003; Wobus et al., 2004).

\subsection{Disinfectants and Cleaning Agents}

Five disinfectant and/or cleaning agents used in this study included one sodium hypochlorite product, two quaternary ammonium compound products (both in ready-to-use (RTU) concentrations) and two accelerated hydrogen peroxide products (one in concentrate, the other in 
RTU concentration) (Table 4). The disinfectant products were selected because they are currently being used in health care facilities in British Columbia, Canada. All disinfectant products were tested within the manufacturer's expiry date. The products were stored at room temperature.

The following products were selected for this study:

1. Sodium hypochlorite: Domestic Miraclean Bleach (Clear Tech Industries Inc., Richmond, BC, Canada) $-5.4 \%$ available chlorine

2. Quaternary ammonium compound (RTU): CaviCide (Metrex Research Corporation, order no. 11-1000, DIN 02161656) - $0.28 \%$ diisobutylphenoxyethoxyethyl dimethyl benzyl ammonium chloride, $17.20 \%$ isopropanol

3. Quaternary ammonium compound (RTU): $\mathbf{T}^{3} \mathbf{6}$ Disinfectant (Alda Pharmaceuticals Corp., DIN 02231344) - $0.28 \%$ o-phenylphenol, $0.20 \%$ benzalkonium chloride, $0.01 \%$ chlorhexidine gluconate, $70 \%$ ethanol

4. Accelerated hydrogen peroxide (RTU): Virox 5 (Virox Technologies Inc., DIN $02239775)-0.5 \%$ accelerated hydrogen peroxide

5. Accelerated hydrogen peroxide (concentrate): Accel (Virox Technologies Inc., DIN 02245061) $-7.0 \%$ accelerated hydrogen peroxide

The disinfectant products were ordered directly from the company to avoid any possible tampering or chemical degradation. Disinfectant solutions were diluted at room temperature using distilled, deionized water, and vortexed for 60 seconds prior to use. For experiments which involved concentrated disinfectants, dilutions of the disinfectants were always prepared 
fresh on the day of the experiment and used within 2 hours of the start of each experiment. RTU disinfectants were used, undiluted, during experiments. Manufacturer recommendations listed on the product labelling such as pre-cleaning and/or pre-rinsing were not followed (Table 5).

\subsection{Neutralizer}

A neutralizer was used to arrest the activity of the disinfectant and to avoid cytotoxicity to the cell culture (Aidaros \& Gaudin, 1983; Sattar et al., 2003). Disinfectants that have not been diluted or neutralized may well affect the cell viability, preventing the development of cytopathic effects and allowing the virus to appear as though it were inactivated (Aidaros \& Gaudin, 1983). Another possible option was to use the gel filtration method to remove the disinfectant from the virus after contact in order to reduce chemical cytotoxicity of the cell culture; however, this method may not necessarily eliminate disinfectant cytotoxicity and may actually expose the host cell to the disinfectant for a longer period of time (Aidaros \& Gaudin, 1983; ASTM International, 1998; Sattar et al., 2003). Based on these observations, it was decided that a neutralizer would be selected and used to arrest the virucidal activity of the disinfectant and/or cleaning agent immediately at the end of the defined contact time. Neutralization was accomplished through a combination of chemical neutralization and serial dilutions.

\subsubsection{Sodium Thiosulphate Neutralizer}

Sodium thiosulphate pentahydrate $\left(\mathrm{Na}_{2} \mathrm{~S}_{2} \mathrm{O}_{3} \cdot 5 \mathrm{H}_{2} \mathrm{O}\right)$ (BDH Chemicals, Toronto, Canada) was prepared (2.5\% concentration) and used as a neutralizer for sodium hypochlorite "Domestic Miraclean” bleach concentrations selected for this study (Table A1) (Association of Official Analytical Chemists, 1975). The neutralizer was prepared by dissolving $12.5 \mathrm{~g}$ of sodium 
thiosulphate pentahydrate in $500 \mathrm{~mL}$ of distilled, deionized water and filtering the preparation through a MF75 membrane filtration unit (75 mm diameter, $0.2 \mu \mathrm{m}$ pore size) (Nalgene, catalog \#28199-155) to sterilize the solution. An antibiotic and antimycotic solution consisting of

Fungizone ${ }^{\circledR}$ amphotericin $\mathrm{B}$, gentamicin reagent solution and penicillin $\mathrm{G}$ potassium salt was incorporated into the neutralizer solution (per $500 \mathrm{~mL}$ of neutralizer) which was then stored at $4{ }^{\circ} \mathrm{C}$.

\subsubsection{Letheen Broth Neutralizer}

Letheen broth (HiMedia Laboratories Pvt. Ltd. India, ref MU165-500G, catalog \#95039-384) at $10.3 \%$ concentration with $2.0 \%$ sodium thiosulphate was used as a neutralizer to arrest the activity of QUAT and AHP (Table A1). The neutralizer was prepared by dissolving $51.4 \mathrm{~g}$ letheen broth powder and $10 \mathrm{~g}$ of sodium thiosulphate pentahydrate to $500 \mathrm{~mL}$ of distilled, deionized water and passed through a MF75 membrane filtration unit (75 mm diameter, $0.2 \mu \mathrm{m}$ pore size) to sterilize the solution. An antibiotic and antimycotic solution consisting of Fungizone ${ }^{\circledR}$ amphotericin $B$, gentamicin reagent solution and penicillin $\mathrm{G}$ potassium salt was incorporated into the solution (per $500 \mathrm{~mL}$ of neutralizer) which was then stored at $4{ }^{\circ} \mathrm{C}$.

\subsection{Additional Control Tests for Virucidal Activity}

Additional control tests were conducted at the beginning of the study because there was a possibility that either the test substance/formulation (disinfectant and/or cleaning agent) or the neutralizer, or a combination of both mixtures, affected the susceptibility of the host cells to the virus during plaque assay experiments. Testing of the test substance/formulation, neutralizer and the combination of both mixtures was conducted in accordance with the protocols reported by 
Sattar et al. (2003). The control tests were run once prior to the start of each selected disinfectant, surrogate virus and cell line, and were not repeated as long as the same conditions (cell line, virus, disinfectant concentration, neutralizer) and methods were used during testing. The standard procedures used in the study were the same as the procedures used to carry out control tests. Two types of control tests were conducted in triplicate: cytotoxicity control test and virus infectivity/interference control test (Sattar et al., 2003).

\subsubsection{Cytotoxicity Control Test}

The cytotoxicity control test (Figure 5) was used for the following reasons: (a) to determine at which test formulation concentration and/or dilution produced no cytotoxicity in the cell line, (b) to assess if the neutralizer reduces or enhances cytotoxicity of the cell line, and (c) to assess if the presence of the soil load mixture reduces or enhances cytotoxicity of the cell line (Sattar et al., 2003). Cytotoxicity was determined by the plaque assay method.

Each selected test formulation (disinfectant) concentration and reagent to be tested in the study was first diluted in a $1 / 20$ dilution $(50 \mu \mathrm{L}$ test formulation and $950 \mu \mathrm{L}$ EBSS/neutralizer $)$ in a sterile plastic test tube followed by one 10-fold serial dilution (and if necessary, more serial dilutions were conducted as needed) in alpha-MEM (167 $\mu \mathrm{L}$ of test dilution in $1.5 \mathrm{~mL}$ alphaMEM) (Sattar et al., 2003).

The following combinations (for the 1/20 dilution) were selected to determine cytotoxicity:

1. Test cytotoxicity of disinfectant without neutralizer $-50 \mu \mathrm{L}$ test formulation and $950 \mu \mathrm{L}$ EBSS; 
2. Test cytotoxicity of disinfectant with neutralizer and assess neutralizer strength $-50 \mu \mathrm{L}$ test formulation and $950 \mu \mathrm{L}$ neutralizer;

3. Test cytotoxicity of neutralizer alone $-1000 \mu \mathrm{L}$ neutralizer;

4. Test cytotoxicity of soil load mixture $-50 \mu \mathrm{L}$ of soil load mixture and $950 \mu \mathrm{L}$ EBSS;

5. Control $-1000 \mu$ L EBSS.

After removal of growth medium, $500 \mu \mathrm{L}$ of the test dilution (from the $10^{-1}$ dilution) was transferred to each well (in triplicate) containing a confluent monolayer of the cell line and incubated at room temperature for 1 hour (Sattar et al., 2003). After 1 hour incubation, the test dilution was removed and an agarose overlay mixture (at $47^{\circ} \mathrm{C}$ ) consisting of equal volumes of $1.25 \%$ SeaPlaque agarose and $2 \mathrm{X}$ alpha-MEM with $10 \%$ FBS was added to each well plate $\left(2 \mathrm{~mL}\right.$ ) and incubated at $37^{\circ} \mathrm{C}$ in a $\mathrm{CO}_{2}$ incubator for $36-40$ hours prior to the addition of a second agarose overlay mixture (at $56^{\circ} \mathrm{C}$ ) containing equal volumes of $1.25 \%$ SeaKem agarose, $0.1 \%$ neutral red and 2X alpha-MEM with $10 \%$ FBS (Wobus et al., 2004). The 6-well plates were incubated for a further 24 hours prior to being examined with a microscope for any visible cytotoxicity (Sattar et al., 2003).

\subsubsection{Interference with Virus Infectivity Control Test}

The interference with virus infectivity control test (Figure 6) was conducted to determine if the test formulation, neutralizer, a combination of test formulation and neutralizer, or the soil load mixture could reduce or enhance the ability of the surrogate virus to infect and replicate in host cells (Sattar et al., 2003). The ability of the virus to infect or replicate in the cell culture may be affected and therefore may interfere with the estimation of the virucidal activity of the 
disinfectant (Sattar et al., 2003). The interference with virus infectivity control test was similar to the cytotoxicity condition test with the addition of a selected challenge virus. Interference with virus infectivity was determined by plaque assay.

A test run with the selected surrogate virus in the absence of the test formulation was first done to determine a countable range of infective units (Sattar et al., 2003). The countable range of between 20 and 30 infective units (virus plaques) for both MNV-1 and FCV was selected after serial dilution of the virus inoculum followed by plaque assay. This countable range of infective units was used for all control tests for interference with virus infectivity.

Each selected test formulation (disinfectant) concentration and reagent was first diluted to a 1/20 dilution $(50 \mu \mathrm{L}$ test formulation and $950 \mu \mathrm{L}$ EBSS/neutralizer) in a sterile $5 \mathrm{~mL}$ polypropylene round-bottom test tube (BD Falcon, catalog \#352063, $12 \times 75 \mathrm{~mm}$ style) followed by one 10 -fold dilution (and if necessary, more serial dilutions were conducted as needed) in alpha-MEM (167 $\mu \mathrm{L}$ of test dilution in $1.5 \mathrm{~mL}$ alpha-MEM) (Sattar et al., 2003).

The following combinations (for the $1 / 20$ dilution) were selected to determine possible interference from the test formulation (disinfectant), neutralizer, or a combination of both:

1. Test interference with virus infectivity by the disinfectant without neutralizer $-50 \mu \mathrm{L}$ test formulation and $950 \mu \mathrm{L}$ EBSS;

2. Test interference with virus infectivity by the disinfectant with neutralizer $-50 \mu \mathrm{L}$ test formulation and $950 \mu \mathrm{L}$ neutralizer;

3. Test interference with virus infectivity by the neutralizer alone $-1000 \mu \mathrm{L}$ neutralizer; 
4. Test interference with virus infectivity by the soil load mixture $-50 \mu \mathrm{L}$ of soil load mixture and $1000 \mu \mathrm{L}$ EBSS

5. Control $-1000 \mu \mathrm{L}$ EBSS.

Growth medium (alpha-MEM) in 6-well plates was removed before $500 \mu \mathrm{L}$ of the test dilution (from the $10^{-1}$ dilution) containing 20-30 infective units of challenge virus was transferred to each of three wells in the plate containing a confluent monolayer of the cell line. The total volume of challenge virus and test dilution should be $500 \mu \mathrm{L}$ for each well plate (e.g., $450 \mu \mathrm{L}$ of test dilution and $50 \mu \mathrm{L}$ of challenge virus containing between 20 and 30 infective units). The inoculated well plates were incubated at room temperature for 1 hour (Sattar et al., 2003). After one hour incubation, the test dilution was removed and a $47^{\circ} \mathrm{C}$ agarose overlay mixture consisting of equal volumes of $1.25 \%$ SeaPlaque agarose and $2 \mathrm{X}$ alpha-MEM with $10 \%$ FBS was added to each well plate $(2 \mathrm{~mL})$ and incubated at $37^{\circ} \mathrm{C}$ in a $\mathrm{CO}_{2}$ incubator for $36-40$ hours (for MNV-1) or 40-45 hours (for FCV) prior to the addition of a $56{ }^{\circ} \mathrm{C}$ second agarose overlay mixture containing equal volumes of $1.25 \%$ SeaKem agarose, $0.1 \%$ neutral red and $2 \mathrm{X}$ alphaMEM with $10 \%$ FBS (Wobus et al., 2004). The well plates were incubated overnight for up to 24 hours before virus plaques were counted as PFU (per mL) (Sattar et al., 2003).

\subsection{Reagent and Media Solution Preparation}

All reagents and growth medium batches that were prepared were monitored for bacterial contamination using thioglycolate broth. One millilitre of reagent or growth medium was added to each thioglycolate broth tube in duplicate and incubated at $37{ }^{\circ} \mathrm{C}$ (Garcia, 2010). The thioglycolate broth tubes were observed for contamination (e.g., turbidity) every 48 hours for 6 
days (Garcia, 2010). The thioglycolate broth tubes were discarded after 6 days (Garcia, 2010). Thioglycolate broth was prepared in-house by quality control laboratory technologists in the British Columbia Public Health \& Microbiology Reference Laboratories (BCPHMRL).

\subsubsection{HEPES Buffer Solution}

HEPES buffer solution (1 M) (4-(2-Hydroxyethyl)piperazine-1-ethanesulfonic acid N-(2Hydroxyethyl)piperazine-N'-(2-ethanesulfonic acid)) was prepared by adding $47.06 \mathrm{~g}$ of HEPES powder (Sigma, catalog \#H3375-500G) to $200 \mathrm{~mL}$ of deionized water in a $500 \mathrm{~mL}$ bottle. The HEPES solution was vacuum filtered using a Millipak® 20 membrane filter system (Millipore, catalog \#MPL02GH2) with a $0.22 \mu \mathrm{m}$ pore size filter to sterilize the solution. The solution was dispensed in $7 \mathrm{~mL}$ aliquots into sterile $15 \mathrm{~mL}$ Falcon tubes and stored at $4{ }^{\circ} \mathrm{C}$ until use (Schmidt \& Emmons, 1989).

\subsubsection{Crystal Violet Solution}

Crystal violet solution at $0.1 \%$ was prepared by adding $2.10 \mathrm{~g}$ citric acid (AnalaR, BDH Chemicals) and $0.10 \mathrm{~g}$ of crystal violet (Fisher Scientific, \#C-581) into a $100 \mathrm{~mL}$ beaker containing $100 \mathrm{~mL}$ deionized water (Garcia, 2010). The reagents were dissolved prior to being filtered into a sterile container using Whatman No. 1 filter paper in a funnel. The crystal violet solution was stored at $4{ }^{\circ} \mathrm{C}$. 


\subsubsection{Penicillin G Potassium Salt Solution}

Penicillin G potassium salt (Sigma, catalog \#P7794-1MU) at $4 \times 10^{6}$ units was dissolved in $200 \mathrm{~mL}$ of distilled, deionized water for a final concentration of 20,000 units/mL (Schmidt \& Emmons, 1989). The penicillin $\mathrm{G}$ solution was dispensed as $5 \mathrm{~mL}$ aliquots into sterile $15 \mathrm{~mL}$ Falcon tubes followed by storage at $-20^{\circ} \mathrm{C}$ until use.

\subsubsection{X alpha-MEM Solution}

2X alpha-MEM was prepared by adding $10.08 \mathrm{~g}$ of alpha-MEM powder (Gibco, catalog \#12000063) and $2.2 \mathrm{~g}$ of sodium bicarbonate (Sigma, catalog \#S5761-500G) to $500 \mathrm{~mL}$ of sterile distilled, deionized water and stirring the media with a stir bar until the MEM powder was completely dissolved (Table A2). A MF75 filtration unit with a $0.20 \mu \mathrm{m}$ pore size filter was used to sterilize the growth medium (Schmidt \& Emmons, 1989). The 2X alpha-MEM solution was stored at $4{ }^{\circ} \mathrm{C}$ until use. An antibiotic and antimycotic solution consisting of Fungizone ${ }^{\circledR}$ amphotericin B, gentamicin reagent solution, penicillin $G$ potassium salt and HEPES buffer solution was incorporated into the solution (per $500 \mathrm{~mL}$ of $2 \mathrm{X}$ alpha-MEM) prior to use and stored at $4{ }^{\circ} \mathrm{C}$.

\subsubsection{Trypsin-Versene Solution}

Trypsin-versene solution was prepared by adding $0.2 \mathrm{~g}$ of dextrose (Fisher Scientific, \# D-16), $0.2 \mathrm{~g}$ of EDTA and $3.0 \mathrm{~g}$ of trypsin 250 (Difco, ref 215240) to $1000 \mathrm{~mL}$ of phosphate-buffered saline (PBS) (Sigma, \#P-3813), and stirred for 30 minutes at room temperature. The solution was sterilized in a MF75 filtration unit with a $0.20 \mu \mathrm{m}$ pore size, dispensed in $25 \mathrm{~mL}$ aliquots in sterile $30 \mathrm{~mL}$ Falcon tubes and stored at $-20{ }^{\circ} \mathrm{C}$ (Schmidt \& Emmons, 1989). 


\subsubsection{Alpha-MEM Solution}

Alpha-MEM was prepared by adding $100.8 \mathrm{~g}$ of alpha-MEM powder (Gibco, catalog \#12000063) and 22.0 g of sodium bicarbonate (Sigma, catalog \#S5761-500G) to $10 \mathrm{~L}$ of sterile distilled, deionized water and stirring the media with a stir bar at room temperature until the ingredients were completely dissolved (Table A2). A Millipak® 20 membrane filter system with a $0.22 \mu \mathrm{m}$ pore size filter was used to sterilize the growth medium (Schmidt \& Emmons, 1989). AlphaMEM was dispensed in $500 \mathrm{~mL}$ aliquots in $500 \mathrm{~mL}$ sterile glass bottles and stored at $4{ }^{\circ} \mathrm{C}$ until use. An antibiotic and antimycotic solution consisting of Fungizone® amphotericin B, gentamicin reagent solution, penicillin $\mathrm{G}$ potassium salt and HEPES buffer solution was prepared separately and incorporated into the MEM solution (per $500 \mathrm{~mL}$ of alpha-MEM) prior to use and stored at $4{ }^{\circ} \mathrm{C}$.

\subsubsection{Fungizone® Amphotericin B Solution}

Fungizone ${ }^{\circledR}$ amphotericin B (Gibco, \#15290-018) is an antimycotic solution that is often used for treating clinical specimens or added to cell culture media (Schmidt \& Emmons, 1989). Fungizone ${ }^{\circledR}$ amphotericin B solution $(250 \mu \mathrm{g} / \mathrm{mL})$ was incorporated into growth medium at $5 \mathrm{~mL}$ per $500 \mathrm{~mL}$ of alpha-MEM growth medium and at $10 \mathrm{~mL}$ per $500 \mathrm{~mL}$ of $2 \mathrm{X}$ alpha-MEM growth medium. Fungizone $®$ amphotericin $\mathrm{B}$ solution was stored at $-20{ }^{\circ} \mathrm{C}$ until use and refrigerated upon opening. 


\subsubsection{Gentamicin Reagent Solution}

Gentamicin reagent solution (Gibco, \#15710-064) (10 mg/mL) was added to growth medium at $2.5 \mathrm{~mL}$ per $500 \mathrm{~mL}$ of alpha-MEM growth medium and $5.0 \mathrm{~mL}$ per $500 \mathrm{~mL}$ of $2 \mathrm{X}$ alpha-MEM growth medium. Gentamicin Reagent Solution was stored at room temperature until use.

\subsection{Soil Load Mixture}

The soil load mixture (Table A3) was prepared by following the procedure set by Sattar et al. (2003). The soil load consisted of a mixture of tryptone, BSA and bovine mucin. The soil load mixture contained $2 \mathrm{~g}$ of total protein/L (Sattar et al., 2003). All soil load stock solutions were prepared separately. The soil load mixture was used instead of a food or lipid-based soil load to allow for more consistency in the methodology.

To prepare a $500 \mu \mathrm{L}$ virus inoculum containing soil load, $25 \mu \mathrm{L}$ of BSA, $100 \mu \mathrm{L}$ of bovine mucin and $35 \mu \mathrm{L}$ of tryptone were added to $340 \mu \mathrm{L}$ of virus suspension and vortexed together prior to use. The virus suspension with soil load mixture was prepared at the start of each experiment.

\subsubsection{Tryptone}

Tryptone was prepared by adding $0.5 \mathrm{~g}$ of BD Bacto ${ }^{\mathrm{TM}}$ tryptone $(\mathrm{BD}$, catalog \#211705, Difco Laboratories, Sparks, MD, 21152, USA) into 10 mL of pH 7.2 PBS (Gibco, catalog \#20012-027) (Sattar et al., 2003). The tryptone solution was sterilized through a MF75 filtration unit with a $0.20 \mu \mathrm{m}$ pore size, followed by addition to the cryovials and stored at $-20^{\circ} \mathrm{C}$ for long-term storage (Sattar et al., 2003). 


\subsubsection{Bovine Mucin}

Bovine mucin was prepared by adding $0.04 \mathrm{~g}$ of bovine mucin (type 1 bovine submaxillary glands) (Sigma, catalog \#M-4503-100MG) to $10 \mathrm{~mL}$ of $\mathrm{pH}$ 7.2 PBS. The bovine mucin solution was sterilized through a MF75 filtration unit with a $0.20 \mu \mathrm{m}$ pore size, into $2 \mathrm{~mL}$ cryovials and stored at $-20^{\circ} \mathrm{C}$ for long-term storage (Sattar et al., 2003).

\subsubsection{Bovine Serum Albumin}

BSA was prepared by adding $0.5 \mathrm{~g}$ of BSA (Sigma, catalog \#B-4287-5G) to $10 \mathrm{~mL}$ of $\mathrm{pH} 7.2$ PBS. The BSA solution was sterilized through a MF75 filtration unit with a $0.20 \mu \mathrm{m}$ pore size, followed by transfer of aliquots to $2 \mathrm{~mL}$ cryovials and subsequent storage at $-20{ }^{\circ} \mathrm{C}$ (Sattar et al., 2003).

\subsubsection{SeaPlaque and SeaKem Agarose Overlay}

A $1.25 \%$ SeaPlaque agarose (Lonza, catalog \#50101) overlay mixture was prepared by mixing equal volumes of $2.5 \%$ SeaPlaque agarose with $2 \mathrm{X}$ alpha-MEM supplemented with $10 \%$ FBS (e.g., $50 \mathrm{~mL}$ solution of $2.5 \%$ SeaPlaque agarose mixed with $45 \mathrm{~mL}$ of $2 \mathrm{X}$ alpha-MEM and $5 \mathrm{~mL}$ of FBS) (Bidawid et al., 2003; Wobus et al., 2004). SeaPlaque agarose powder was added to distilled, deionized water at $0.25 \mathrm{~g} / 10 \mathrm{~mL}$ in a $100 \mathrm{~mL}$ (4 oz.) sterile, plastic specimen container (Kendall, Precision ${ }^{\mathrm{TM}}$ specimen container, code \#2200SA), sealed in the container, and carefully microwaved (at 5-15 second increments, for up to 60 seconds total) to melt the SeaPlaque, making sure the agarose did not boil over. Once microwaved, the clear, liquid agarose was immediately put in a $47^{\circ} \mathrm{C}$ water bath along with the solution of $2 \mathrm{X}$ alpha-MEM 
with $10 \%$ FBS to equilibrate. Both solutions were taken out of the water bath and mixed into one bottle immediately before overlaying the well plates with agarose (Wobus et al., 2004).

A $1.25 \%$ SeaKem agarose (Lonza, catalog \#50001) overlay mixture was prepared by mixing equal volumes of $2.5 \%$ SeaKem agarose with 2X alpha-MEM supplemented with $10 \%$ FBS and $0.1 \%$ neutral red solution (Sigma, catalog \#57993-100ML-F) that is $1 / 10$ of the total volume of overlay mixture (e.g., a $100 \mathrm{~mL}$ total volume of $1.25 \%$ SeaKem agarose overlay should contain $50 \mathrm{~mL}$ solution of $2.5 \%$ SeaKem agarose mixed with $36 \mathrm{~mL}$ of $2 \mathrm{X}$ alpha-MEM, $4 \mathrm{~mL}$ of FBS and $10 \mathrm{~mL}$ of a $0.1 \%$ neutral red solution) (Bidawid et al., 2003; Wobus et al., 2004). SeaKem agarose powder was added to distilled, deionized water at $0.25 \mathrm{~g} / 10 \mathrm{~mL}$ in a sterile, plastic specimen container and carefully microwaved to melt the SeaKem (at 5-15 second increments, for up to 60 seconds total). Once microwaved, the clear, liquid agarose was immediately put in a $56{ }^{\circ} \mathrm{C}$ water bath along with the solution of $2 \mathrm{X}$ alpha-MEM supplemented with $10 \% \mathrm{FBS}$ and neutral red for temperature equilibration. Both solutions were taken out of the water bath and mixed into one bottle immediately before overlaying the well plates with agarose (Wobus et al., 2004).

\subsection{Statistical Analysis}

Tests were done in triplicate, and data (number of plaques counted) was calculated as mean $\log _{10}$ reduction $(\mathrm{PFU} / \mathrm{mL})$ and plotted on graphs as mean $\log _{10}$ reduction against disinfectant concentration $(\mathrm{ppm})$ or contact time (min). Disinfectants and/or cleaning agents were classified using a $\log _{10}$ reduction criterion to determine their efficacy in inactivating MNV-1 and FCV, or

both. Results were expressed in the form of descriptive analysis. Results of the cytotoxicity and interference with virus infectivity control tests (plaque forming units or PFU) were analysed for 
statistically significant differences $(\mathrm{p}<0.05)$ by single factor Analysis of Variance (ANOVA) and for treatments that were significantly different, Tukey's Honestly Significant Difference (HSD) Test was used to determine which treatments were significantly different. The PFU results were compared to the control. The effect of drying on the viral titer was analysed using a two-sample t-test (assuming unequal variances). All statistics were performed using Microsoft Office Excel 2007. 
Table 4. List of disinfectants tested, manufacturers, active ingredients, concentrations tested and classification.

\begin{tabular}{|c|c|c|c|c|c|}
\hline $\begin{array}{l}\text { Disinfectant } \\
\text { name }\end{array}$ & Manufacturer & $\begin{array}{l}\text { Active } \\
\text { ingredient(s) }\end{array}$ & $\begin{array}{l}\text { Dilution(s) } \\
\text { tested }\end{array}$ & $\begin{array}{l}\text { Concentration(s) } \\
\text { tested (ppm) }\end{array}$ & $\begin{array}{l}\text { Classification } \\
\text { and type }\end{array}$ \\
\hline $\begin{array}{l}\text { Domestic } \\
\text { Miraclean } \\
\text { Bleach }\end{array}$ & $\begin{array}{l}\text { ClearTech } \\
\text { Industries Inc., } \\
\text { Richmond, BC, } \\
\text { Canada }\end{array}$ & $5.4 \%$ chlorine & $\begin{array}{l}1 / 10 \\
1 / 20 \\
1 / 40 \\
1 / 80 \\
1 / 160 \\
1 / 320 \\
1 / 640 \\
1 / 1280 \\
\end{array}$ & $\begin{array}{l}5400 \\
2700 \\
1350 \\
675 \\
338 \\
169 \\
84 \\
42 \\
\end{array}$ & $\begin{array}{l}\text { Disinfectant, } \\
\text { halogen or } \\
\text { chlorine }\end{array}$ \\
\hline Accel & $\begin{array}{l}\text { Virox } \\
\text { Technologies Inc., } \\
\text { Oakville, ON, } \\
\text { Canada }\end{array}$ & $7.0 \% \mathrm{AHP}^{\mathrm{a}}$ & $\begin{array}{l}1 / 2 \\
1 / 4 \\
1 / 10 \\
1 / 20 \\
1 / 40\end{array}$ & $\begin{array}{l}35000 \\
17500 \\
7000 \\
3500 \\
1750\end{array}$ & $\begin{array}{l}\text { Disinfectant, } \\
\text { AHP }\end{array}$ \\
\hline Virox 5 & $\begin{array}{l}\text { Virox } \\
\text { Technologies, } \\
\text { Inc., Oakville, } \\
\text { ON, Canada } \\
\end{array}$ & $0.5 \% \mathrm{AHP}^{\mathrm{a}}$ & $\begin{array}{l}\text { Undiluted, } \\
\text { RTU }^{\mathrm{b}}\end{array}$ & 5000 & $\begin{array}{l}\text { Disinfectant, } \\
\text { AHP }\end{array}$ \\
\hline CaviCide & $\begin{array}{l}\text { Metrex Research } \\
\text { Corp., Orange, } \\
\text { CA, USA }\end{array}$ & $\begin{array}{l}17.2 \% \\
\text { isopropanol, } \\
0.28 \% \text { QUAT }^{\mathrm{c}}\end{array}$ & $\begin{array}{l}\text { Undiluted, } \\
\text { RTU }^{\mathrm{b}}\end{array}$ & 2800 & $\begin{array}{l}\text { Disinfectant, } \\
\text { QUAT }\end{array}$ \\
\hline$T^{3} 6$ & $\begin{array}{l}\text { ALDA } \\
\text { Pharmaceuticals } \\
\text { Corp., New } \\
\text { Westminster, BC, } \\
\text { Canada }\end{array}$ & $\begin{array}{l}70 \% \text { ethanol, } \\
0.28 \% \\
o \text {-phenylphenol, } \\
0.01 \% \\
\text { chlorhexidine } \\
\text { gluconate, } \\
0.20 \% \text { QUAT } \\
\end{array}$ & $\begin{array}{l}\text { Undiluted, } \\
\text { RTU }^{\mathrm{b}}\end{array}$ & 2000 & $\begin{array}{l}\text { Disinfectant, } \\
\text { QUAT-alcohol }\end{array}$ \\
\hline
\end{tabular}

${ }^{\mathrm{a}} \mathrm{AHP}$ refers to accelerated hydrogen peroxide.

${ }^{\mathrm{b}} \mathrm{RTU}$ refers to "ready-to-use".

${ }^{\mathrm{c}} 0.28 \%$ QUAT refers to quaternary ammonium compound containing diisobutylphenoxyethoxyethyl dimethyl benzyl ammonium chloride.

d $0.20 \%$ QUAT refers to quaternary ammonium compound containing benzalkonium chloride. 
Table 5. Manufacturer recommendations for the five disinfectants used in the study.

\begin{tabular}{|c|c|c|c|}
\hline Disinfectant name & $\begin{array}{l}\text { Manufacturer } \\
\text { directions }\end{array}$ & Dilution & Exposure time $(\mathrm{min})$ \\
\hline $\begin{array}{l}\text { Domestic Miraclean } \\
\text { Bleach }\end{array}$ & $\mathrm{NA}$ & NA & NA \\
\hline \multirow[t]{2}{*}{ Accel* } & $\begin{array}{l}\text { Disinfect } \\
\text { contaminated surface } \\
\text { with blood or body } \\
\text { fluids, non-critical } \\
\text { medical devices, } \\
\text { equipment, non- } \\
\text { porous hard surfaces }\end{array}$ & $\begin{array}{l}64 \mathrm{~mL} \text { per } 1000 \mathrm{~mL} \\
\text { water }\end{array}$ & 5 \\
\hline & $\begin{array}{l}\text { Environmental } \\
\text { surfaces }{ }^{b}\end{array}$ & $\begin{array}{l}16 \mathrm{~mL} \text { per } 1000 \mathrm{~mL} \\
\text { water }\end{array}$ & 0.5 \\
\hline \multirow[t]{2}{*}{ Virox 5* } & $\begin{array}{l}\text { Disinfect } \\
\text { contaminated surface } \\
\text { with blood or body } \\
\text { fluids, non-critical } \\
\text { medical devices, } \\
\text { equipment, non- } \\
\text { porous hard surfaces }\end{array}$ & Full strength & 5 \\
\hline & $\begin{array}{l}\text { Environmental } \\
\text { surfaces }\end{array}$ & Full strength & 0.5 \\
\hline \multirow[t]{3}{*}{ CaviCide* } & $\begin{array}{l}\text { Instrument } \\
\text { decontamination }^{c} \\
\text { spray }^{c}\end{array}$ & Full strength & 3 \\
\hline & $\begin{array}{l}\text { Manual cleaner for } \\
\text { dirty objects }\end{array}$ & $\begin{array}{l}30 \mathrm{~mL} \text { per } 1000 \mathrm{~mL} \\
\text { water }\end{array}$ & \\
\hline & Non-critical objects ${ }^{\mathrm{e}}$ & Full strength & 3 \\
\hline \multirow[t]{3}{*}{$\mathrm{T}^{3} 6^{*}$} & $\begin{array}{l}\text { Hard surfaces }{ }^{\mathrm{f}}, \text { food } \\
\text { contact surfaces }^{\mathrm{g}}\end{array}$ & Full strength & 5 \\
\hline & $\begin{array}{l}\text { Furniture, porous } \\
\text { surfaces }\end{array}$ & Full strength & Air dry \\
\hline & Instruments $^{i}$ & Full strength & 5 \\
\hline
\end{tabular}

NA refers to not applicable. *Soiled surface or object requires cleaning prior to disinfection.

${ }^{\mathrm{a}}$ Apply to surface using cloth or disposable wipe for specific exposure time, wipe surface dry or rinse.

${ }^{\mathrm{b}}$ Apply to contaminated area for specific exposure time, wipe surface dry.

${ }^{\mathrm{c} S}$ Spray solution onto instrument, allow it to remain wet for 3 minutes, rinse.

${ }^{\mathrm{d}}$ Pre-rinse dirty objects under running tap water, remove debris, place object into disinfectant, scrub until visibly clean, rinse.

${ }^{\mathrm{e}}$ Pre-clean objects and rinse, submerge into CaviCide solution for 3 minutes, remove and rinse or wipe dry.

${ }^{\mathrm{f}}$ Spray surface until wet, wait 5 minutes.

${ }^{\mathrm{g}}$ Spray surface until wet, wait 5 minutes, rinse with potable water.

${ }^{\mathrm{h}}$ Wipe surface using paper towel with disinfectant, allow to air dry.

${ }^{\text {iS }}$ Soak instrument for 5 minutes, dry afterward. 


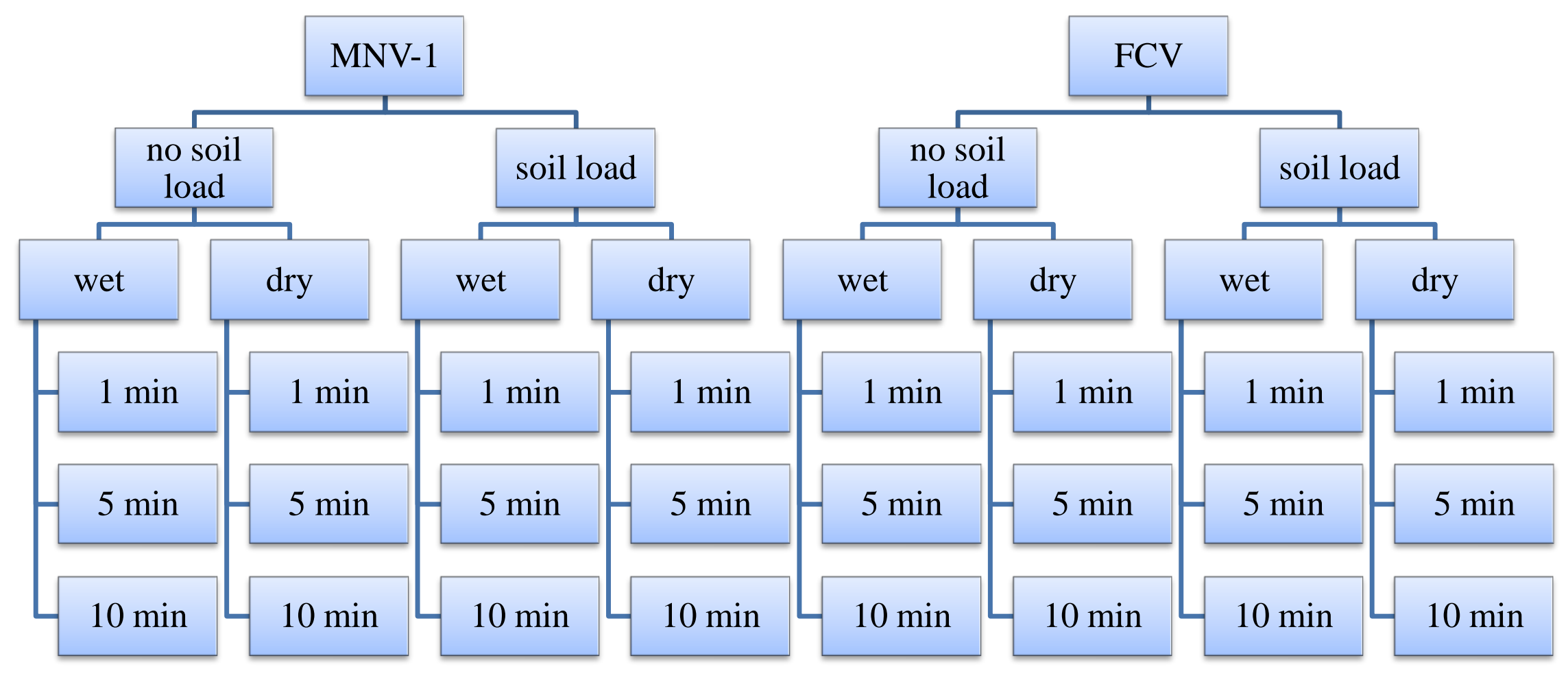

Figure 3. Experimental design flowchart of the study including surrogate viruses MNV-1 and FCV, soil load versus no soil load, wet versus dry load conditions, and contact time. 


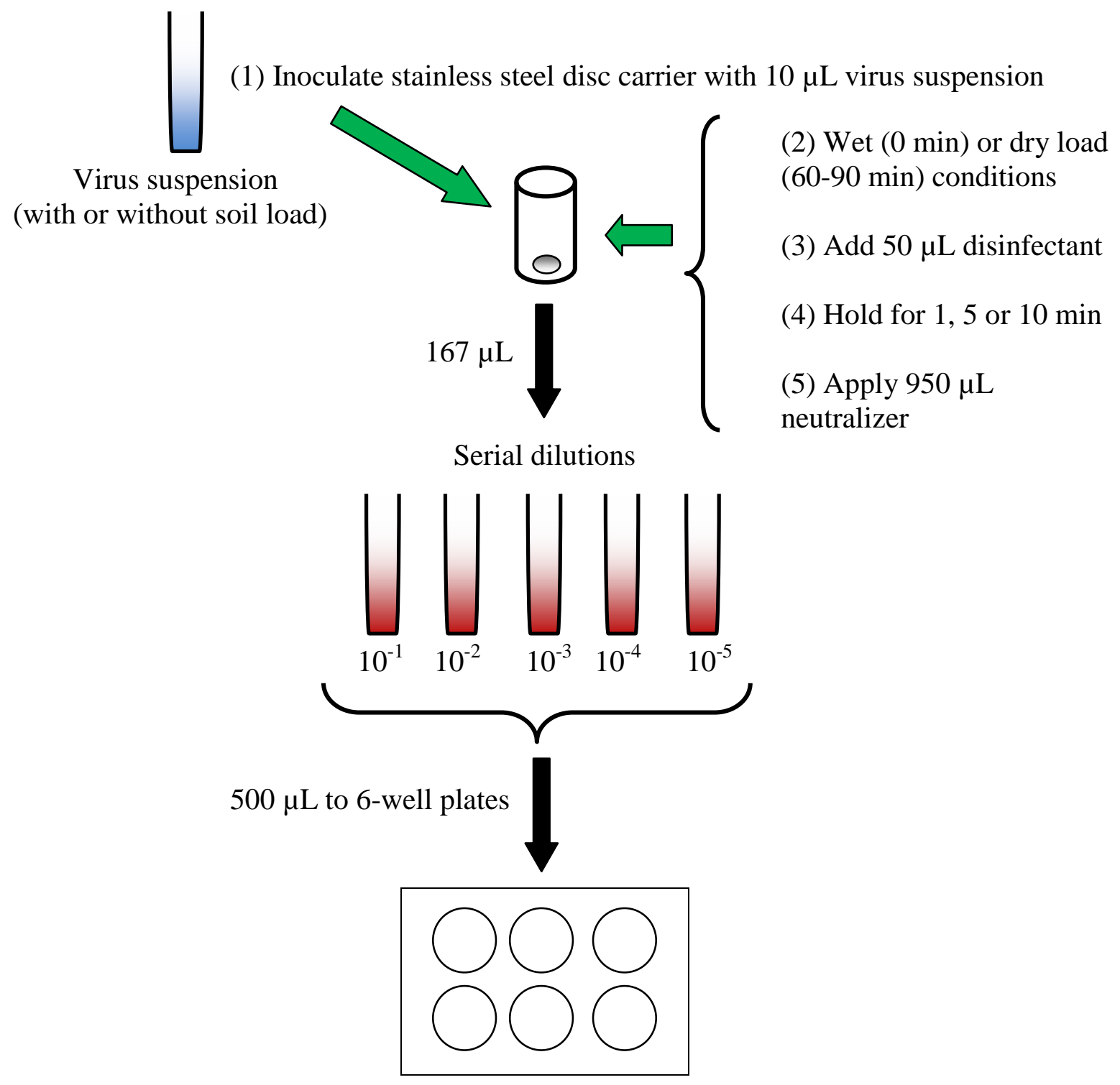

Incubate for 1 hour at room temperature

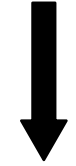

(1) Add first agarose overlay - incubate $36-40$ hours (MNV-1) or 40-45 hours (FCV) at $37^{\circ} \mathrm{C}$

(2) Add second agarose overlay (with neutral red) - incubate 24 hours at $37^{\circ} \mathrm{C}$

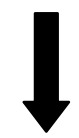

Count virus plaques as plaque forming units $(\mathrm{PFU} / \mathrm{mL})^{*}$

Figure 4. QCT-2 method for virus inactivation and plaque assay method.

*Plaques were visualized at 5-8 and 24 hours for MNV-1, and at 24 hours for FCV. 
(1)

Disinfectant

$50 \mu \mathrm{L}$ disinfectant

$+$

$950 \mu \mathrm{L}$ EBSS
(2)

Disinfectant + Neutralizer
$50 \mu \mathrm{L}$
disinfectant
$+$
$950 \mu \mathrm{L}$
neutralizer

(3)

Neutralizer

$1000 \mu \mathrm{L}$

neutralizer
(4)

Soil Load

(5)

Control

neutralizer

$167 \mu \mathrm{L}$

1.5 mL MEM test tube

$10^{-1}$ dilution

$500 \mu \mathrm{L}$ to 6 -well plates

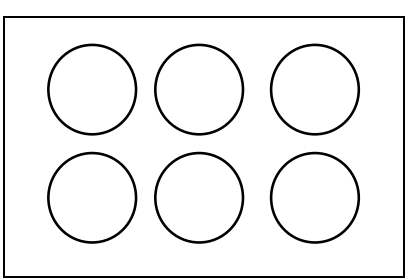

Incubate 1 hour at room temperature

(1) Add first agarose overlay - incubate $36-40$ hours at $37^{\circ} \mathrm{C}$

(2) Add second agarose overlay (with neutral red) - incubate 24 hours at $37^{\circ} \mathrm{C}$

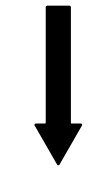

View for visible cytotoxicity

Figure 5. Cytotoxicity control test. 
(1)

Disinfectant

$50 \mu \mathrm{L}$ disinfectant

$+$

$950 \mu \mathrm{L}$ EBSS
(2)

Disinfectant +

Neutralizer

$$
\begin{gathered}
50 \mu \mathrm{L} \text { disinfectant } \\
+ \\
950 \mu \mathrm{L} \text { neutralizer }
\end{gathered}
$$

(3)

Neutralizer

$1000 \mu \mathrm{L}$ neutralizer

$50 \mu \mathrm{L}$ soil load $950 \mu \mathrm{L}$ EBSS
$+$

(4)

Soil Load

Control

$1000 \mu \mathrm{L}$

EBSS
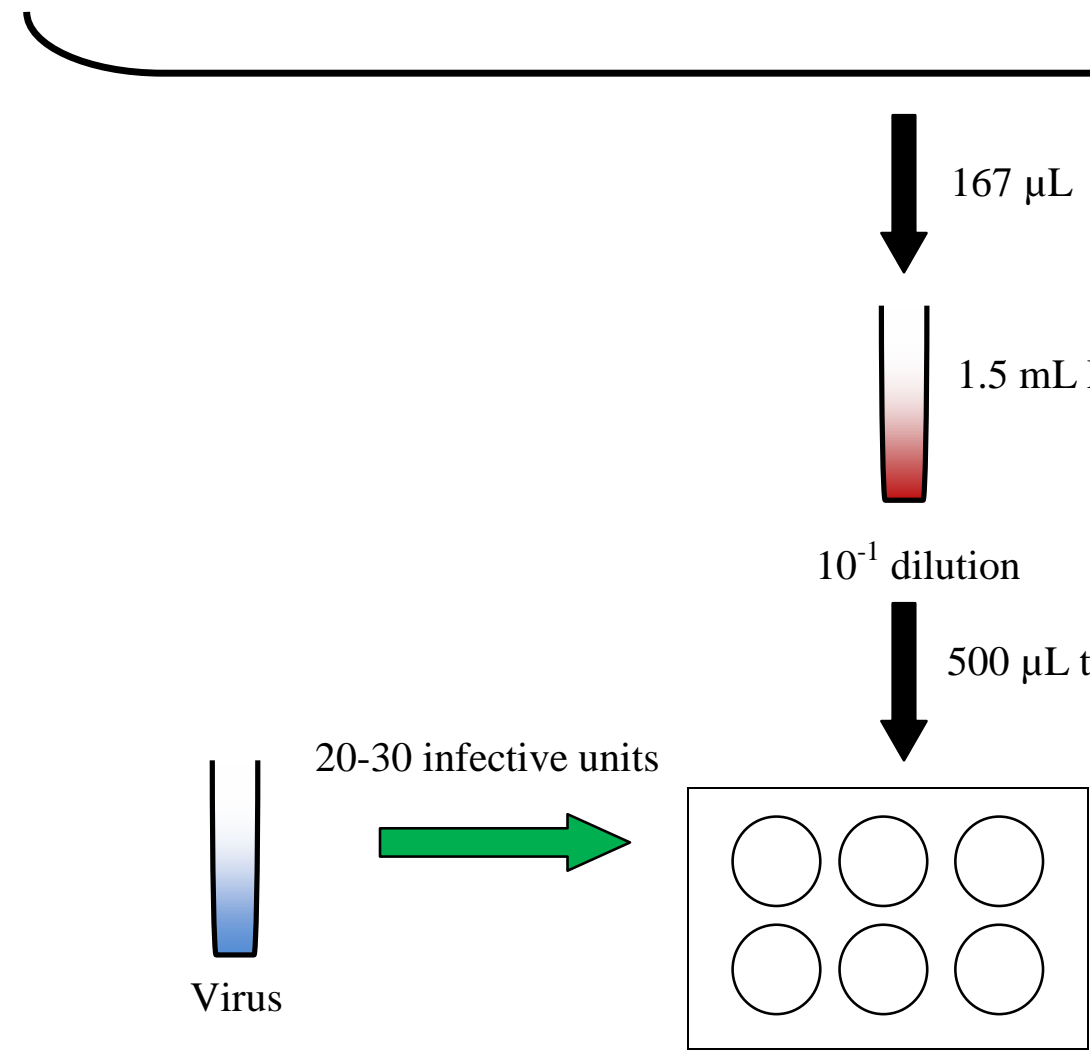

Incubate 1 hour at room temperature

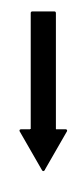

(1) Add first agarose overlay - incubate $36-40$ hours (MNV-1) or 40-45 hours (FCV) at $37{ }^{\circ} \mathrm{C}$

(2) Add second agarose overlay (with neutral red) - incubate 24 hours at $37^{\circ} \mathrm{C}$

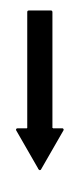

Count virus plaques as plaque forming units $(\mathrm{PFU} / \mathrm{mL}) *$

Figure 6. Interference with virus infectivity control test.

*Plaques were visualized at 5-8 and 24 hours for MNV-1, and at 24 hours for FCV. 


\section{CHAPTER 3 Results and Discussion}

Human norovirus is a highly infectious virus capable of producing symptoms such as nausea, abdominal cramps, vomiting and watery diarrhea (Hutson et al., 2004; Nuermberger, 2005; Radford et al., 2004). It is estimated that in a single incident of vomiting, up to $3 \times 10^{7}$ virus particles can be shed in the form of an aerosol (Barker et al., 2004). Norovirus has a low infectious dose, hence individuals ingesting as little as 10 to 100 virus particles may become infected (Caul, 1994; Hutson et al., 2004). There is evidence of viral shedding as monitored by RT-PCR for several weeks despite the resolution of symptoms (Moe, 2009). Norovirus has been known to survive on human hands, environmental surfaces and inanimate objects for long periods of time and may remain infectious for up to 28 days, and has been demonstrated to survive in stool samples for $\leq 14$ days (Hota, 2004; Lamhoujeb et al., 2009; Liu et al., 2009; Marshall \& Bruggink, 2011). The virus is stable under a wide range of temperatures from freezing to $60{ }^{\circ} \mathrm{C}$ (Nuermberger, 2005; Weber et al., 2010). With its low infectious dose and ability to be transmitted through a number of routes including the fecal-oral route, contamination of environmental surfaces plays an important role in prolonging norovirus outbreaks, especially in long-term care facilities (Lopman et al., 2003; Wu et al., 2005). Based on epidemiological investigations of norovirus outbreaks which occurred in hotels, cruise ships, hospitals, long-term care facilities and daycares, norovirus has been shown to be environmentally stable (CalderonMargalit et al., 2005; Hota, 2004; Isakbaeva et al., 2005; Wu et al., 2005). Norovirus originating from food service workers and food handlers who are ill and practice inadequate hand hygiene can also be transmitted directly on to food or environmental surfaces of restaurants, and an estimated $\sim 50 \%$ of all outbreaks of norovirus infection were associated with infected food service workers and handlers (Boxman et al., 2009; Moe, 2009; Widdowson et al., 2005). A 
finger pad study had demonstrated liquid soap to be more effective against norovirus than alcohol-based hand sanitizers containing $62 \%$ ethyl alcohol (Liu et al., 2010). At this time, proper hand hygiene should be practiced through hand washing with soap and water prior to contact, preparation or consumption of foods and beverages (Centers for Disease Control and Prevention, 2011b; Lages et al., 2008). However, it is unknown at the time if alcohol-based hand sanitizers are also effective against human norovirus (Centers for Disease Control and Prevention, 2011c). The CDC recommends sodium hypochlorite (chlorine bleach) or other disinfectant products approved by the United States Environmental Protection Agency (USEPA) for use in reducing the spread of human norovirus from contaminated environmental surfaces, whereas in British Columbia, Canada, the BCCDC recommends sodium hypochlorite (chlorine bleach), AHP or alternative disinfectants that have been pre-approved by the Environmental Health Officer (EHO) (British Columbia Centre for Disease Control, 2011; Centers for Disease Control and Prevention, 2011c; United States Environmental Protection Agency, 2009).

Because human norovirus cannot be cultivated in cell culture, a number of human norovirus studies have relied on real-time RT-PCR to determine the viral titer; however, one of the drawbacks of this method is its inability to distinguish between infectious and non-infectious virus (Baert et al., 2008). Cultivable surrogate viruses of human norovirus such as MNV-1 and FCV have been selected to study the persistence, inactivation and transmission of human norovirus; however, these limitations (e.g., the use of surrogate viruses and the inability of human norovirus to grow in cell culture) may not accurately reflect how human noroviruses behave in terms of environmental survival and susceptibility to disinfectants (Cannon et al., 2006; Weber et al., 2010). As a result, the only established method which can accurately 
quantitate (infectious) virus titers is through plaque assay (Bidawid et al., 2003; Wobus et al., 2004). Both surrogate viruses, although they share the same Calicivirus family as human norovirus, belong to different genera; human norovirus and MNV-1 belong in the Norovirus genus whereas FCV belongs in the Vesivirus genus (Karst, 2010). In addition, human norovirus can be found in genogroups I, II and IV, whereas MNV-1 is categorized in genogroup V (Karst, 2010). Despite sharing biochemical and genetic features such as size, shape and buoyant density characteristics, MNV-1 and human noroviruses are distinct strains (Hsu et al., 2007). MNV-1 and FCV are also distinct viruses in terms of their stability upon exposure to $\mathrm{pH}$, organic solvents, temperature and environmental surface conditions (Cannon et al., 2006). MNV-1 was claimed to be a more suitable surrogate virus than FCV based on its ability to survive in room temperature and at pH 2 to 10 (Cannon et al., 2006). Sodium hypochlorite and alcohols (ethanol, 1-propanol and 2-propanol) are effective against MNV-1 and FCV (Belliot et al., 2008; Gehrke et al., 2004; Magulski et al., 2009; Malik et al., 2006; Whitehead \& McCue, 2010). However, QUATs were not effective against either surrogate virus (Belliot et al., 2008; Whitehead \& McCue, 2010). Iodine was able to inactivate MNV-1 but was ineffective against FCV (Belliot et al., 2008; Lages et al., 2008). AHP, a less harmful and more environmentally favourable alternative to bleach, has shown promise in its effectiveness against FCV; however, it is unknown if AHP would also be effective against MNV-1 (Omidbakhsh \& Sattar, 2006).

The disinfectants that were selected for this study are commonly used in British Columbia health care facilities; however, because of the limited data on the effectiveness of AHP against noroviruses, it was determined that all three types of disinfectants (sodium hypochlorite, AHP and QUATs) would be tested against both surrogates of norovirus, MNV-1 and FCV. The 
purpose of this study was to determine the efficacy of commonly used disinfectants in British Columbia, such as sodium hypochlorite, AHP and QUATs, against both MNV-1 and FCV under the same conditions and methodology. Previous studies have evaluated the effectiveness of different types of disinfectants using either MNV-1 or FCV, but generally not both, in addition to different methodologies (real-time RT-PCR or plaque assay), environmental conditions and contact times, making it difficult to assess the efficacy of the disinfectant product. It was necessary to evaluate the effectiveness of commonly used disinfectants on both surrogate viruses under the same environmental conditions and contact times, and using plaque assay to accurately quantitate infectious virus titer.

The focus was to determine which disinfectants, and at which concentrations and contact times, were most effective in inactivating $\mathrm{MNV}-1$ and $\mathrm{FCV}$, and to determine if there were any differences in terms of disinfectant efficacy towards either surrogate virus. Unlike previous studies which focused on the efficacy of disinfectants on the virus with soil load which had been dried on surfaces, this study evaluated the stability of the virus under both wet and dry load conditions.

The goal was to investigate the effectiveness of the disinfectants on viruses subjected to two environments under wet and dry load conditions. This study was taken one step further to also include two additional conditions by comparing the efficacy of the disinfectant against viruses with soil load versus viruses without a soil load (under wet and dry load conditions). The presence of a soil load can interfere with the biocide, by either interacting with, reducing its effectiveness, or by preventing access to the virus by physically protecting the target virus (Sattar 
et al., 2003). For example, virus under wet load conditions simulated fresh vomitus or liquid stool, whereas virus under dry load conditions simulated vomitus or liquid stool that underwent environmental desiccation on surfaces. The soil load mixture in this study, which consisted of tryptone, BSA and bovine mucin, contained approximately $2 \mathrm{~g}$ of total protein/L which is equivalent to the protein content of a $5 \%$ solution of FBS (Sattar et al., 2003).

Three contact times (1, 5 and 10 minutes), from short to long, were selected to determine whether the length of the exposure times had an effect on the efficacy of the disinfectant. The short contact time of 1 minute was selected for this study because the contact time is generally between 1 and 3 minutes in the field, and 1 minute is the normal drying time when a disinfectant is applied to non-critical environmental surfaces with a wet cloth (Rutala et al., 2006; Sattar et al., 2003). The performance criteria for the disinfectants generally differ from one jurisdiction to another and it is often difficult to determine the relationship between the efficacy of the disinfectant product and its ability to prevent the transmission of infection; however, the criteria range is usually a 2 to $4 \log _{10}$ reduction in the titer of virus infectivity on hard surfaces (Sattar et al., 2003). Although it is currently not known what level of viral reduction would be needed to prevent the spread of norovirus, in this study, the performance criteria for the selected disinfectants was a $4 \log _{10}$ reduction in the virus titer, regardless of the medium the virus was in (e.g., with or without soil load). The virus, with or without soil load, was exposed to the test disinfectant for a selected period of time, followed by the addition of a neutralizer to arrest the activity of the disinfectant. The $\log _{10}$ reduction was determined using plaque assay. 


\subsection{Efficacy of Sodium Hypochlorite (Domestic Miraclean Bleach) against}

\section{MNV-1 and FCV, with and without Soil Load, under Wet and Dry Load Conditions}

The CDC recommends sodium hypochlorite (chlorine bleach) at a concentration of 1000 to 5000 ppm for disinfecting hard, non-porous, environmental surfaces that had been contaminated with norovirus in a health care environment (Centers for Disease Control and Prevention, 2011c).

In this study, Domestic Miraclean Bleach (or sodium hypochlorite) was tested at different concentrations, ranging from 42 to $5400 \mathrm{ppm}$ (or two-fold serial dilutions starting from 1/10 to 1/1280 dilution) (Figure 7), and the results were within ranges noted in previous studies, where sodium hypochlorite at higher concentrations of $1000 \mathrm{ppm}$ was effective against FCV and $2600 \mathrm{ppm}$ for 30 seconds was effective against MNV-1 with $>4 \log _{10}$ reduction (Belliot et al., 2008; Doultree et al., 1999; Whitehead \& McCue, 2010).

At 2700 ppm, MNV-1 without soil load (Figure 7A) was inactivated after a short contact time of 1 minute with 6.8 and $5.9 \log _{10}$ reduction under wet and dry load conditions, respectively, whereas in the presence of a soil load (Figure 7B), the virus was only inactivated at $5400 \mathrm{ppm}$ with 6.4 and $6.7 \log _{10}$ reduction under wet and dry load conditions, respectively. The presence of the soil load interfered with the effectiveness of the sodium hypochlorite solution and in order to fully inactivate MNV-1, doubling the concentration was required to inactivate the virus with the soil load. However, at longer contact times of 5 minutes, the concentration of sodium hypochlorite did not matter, and $1350 \mathrm{ppm}$ was enough to inactivate MNV-1, with or without soil load, at wet and dry load conditions with virus inactivation of $>6.0$ and $\sim 5.5 \log _{10}$ reduction, respectively (Figure 7). At 10 minute contact time, MNV-1 without soil load (Figure 7A) was 
inactivated at $675 \mathrm{ppm}$ with 6.4 and $5.6 \log _{10}$ reduction under wet and dry load conditions, respectively, suggesting that exposure time may be a factor in assessing the efficacy of sodium hypochlorite. Based on these results, it is recommended that the concentration of sodium hypochlorite be at least 5400 ppm (or 1/10 dilution of bleach containing $5.4 \%$ available chlorine). This is particularly important when dealing with norovirus outbreaks involving dried vomitus or stool on environmental surfaces based on the observation that at these concentrations, sodium hypochlorite can readily inactivate norovirus surrogates. Sodium hypochlorite at high concentrations was able to inactivate MNV-1 at all contact times (1, 5 and 10 minutes) under wet and dry conditions, with or without soil load, with $>5.5 \log _{10}$ reduction (Table 10 ).

Sodium hypochlorite was also effective against FCV, showing inactivation at all contact times (1, 5 and 10 minutes) without soil load, and inactivation at 5 and 10 minute contact times in the presence of a soil load (Figure 8). At 5400 ppm, FCV without soil load (Figure 8A) was inactivated after a short contact time of 1 minute with 5.7 and $5.4 \log _{10}$ reduction under wet and dry load conditions, respectively; however in the presence of a soil load (Figure 8B), the disinfectant was only able to inactivate the virus under wet load with $5.5 \log _{10}$ reduction but not under dry load, with only a $3.7 \log _{10}$ reduction. At the 5 and 10 minute contact times, the concentration of sodium hypochlorite did not need to be as high as $5400 \mathrm{ppm}$ in order to inactivate the virus without soil load. Inactivation occurred at $1350 \mathrm{ppm}$ after exposure to the sodium hypochlorite solution for 5 minutes which resulted in a 4.6 and $4.9 \log _{10}$ reduction under wet and dry load, respectively. Regardless of the presence or absence of a soil load, FCV was inactivated at $2700 \mathrm{ppm}$ with $\sim 5.0 \log _{10}$ reduction if an exposure time of at least 5 minutes was used (Table 11). 
Different chlorine concentrations are required to inactivate either MNV-1 or FCV, and that using the same concentrations and contact times for both viruses may not be effective to inactivate both surrogate viruses under the same conditions (particularly with shorter contact times) (Table 9). It was also observed that higher concentrations of sodium hypochlorite, sometimes as much as a two-fold difference, were needed to inactivate FCV (with or without soil load) than MNV-1. This suggests that FCV is more resistant to chlorine than MNV-1, and to inactivate both surrogate viruses, with or without soil load, under the same conditions such as chlorine concentration, longer exposure times of 10 minutes at $1350 \mathrm{ppm}$ or shorter contact times of 5 minutes at higher chlorine concentrations of $2700 \mathrm{ppm}$ were required.

Although 1000 ppm was not tested in this study, these findings are relatively consistent and within range with the $\mathrm{CDC}$ recommendations for interrupting the spread of norovirus which require between 1000 and 5000 ppm of chlorine bleach for disinfection of environmental surfaces (Centers for Disease Control and Prevention, 2011c).

\subsection{Efficacy of Accelerated Hydrogen Peroxide (Accel and Virox 5) against} MNV-1 and FCV, with and without Soil Load, under Wet and Dry Load Conditions

No research to date has been conducted on the effectiveness of AHP (Accel and Virox 5) against MNV-1; however, one study tested the virucidal effects of a $0.5 \%$ ready-to-use strength AHP product, Accel TB, on FCV, and found that after a 1 minute contact time, there was a $>6.25 \log _{10}$ reduction (Omidbakhsh \& Sattar, 2006). 
In this study, Accel, a concentrated AHP product, was tested at concentrations ranging from 1750 to 35000 ppm AHP; and Virox 5, a ready-to-use strength AHP product, was tested at $0.5 \%$ or 5000 ppm AHP against MNV-1 and FCV (Table 9). Accel at the highest concentration of $35000 \mathrm{pm}$ was unable to inactivate MNV-1, with or without soil load under wet and dry load conditions, after 1 minute contact time. At $17500 \mathrm{ppm}$, there was $<1.0 \log _{10}$ reduction for the virus without soil load under wet and dry load conditions after 1 minute contact time.

At 5 minute contact time, Accel inactivated MNV-1 at 35000 ppm, demonstrating a $6.8 \log _{10}$ reduction under wet load, but was unable to inactivate the virus under dry load conditions, with only a $3.8 \log _{10}$ reduction; with a soil load, the disinfectant was also unable to inactivate the virus. At $17500 \mathrm{ppm}$, there was a 3.4 and $2.5 \log _{10}$ reduction under wet and dry load conditions, respectively. After 10 minutes at 35000 ppm, MNV-1 without soil load was inactivated with 6.5 and $5.6 \log _{10}$ reductions under wet and dry load, respectively, and 6.3 and $5.6 \log _{10}$ reductions with soil load under wet and dry load, respectively (Figure 9). In the presence of a soil load, MNV-1 was inactivated after 10 minutes at 35000 ppm AHP under wet and dry load conditions with 6.3 and $5.6 \log _{10}$ reductions, respectively (Table 10).

Accel was tested at concentrations ranging from 219 to 35000 ppm AHP against FCV (Figure 10). In the absence of a soil load, at specific concentrations, FCV was inactivated at all contact times under wet load and at 5 and 10 minute contact time under dry load. Under wet load, Accel at 7000, 1750 and 875 ppm was effective at 1,5 and 10 minute contact times, respectively, against FCV without soil load, with >5.4 $\log _{10}$ reduction; and under dry load, 1750 ppm was enough to inactivate FCV after 5 minutes with $>5.2 \log _{10}$ reduction (Figure 10A). 
In the presence of a soil load, Accel at 7000 ppm was effective against FCV after 5 minutes with 5.1 and $4.8 \log _{10}$ reductions under wet and dry load, respectively, whereas if exposure times were extended to 10 minutes, only $3500 \mathrm{ppm}$ would be needed to inactivate the virus under wet and dry load conditions (Figure 10B). In the absence of a soil load, it was easier to inactivate the virus at lower disinfectant concentrations. In addition, FCV under wet load conditions, especially without soil load, was easier to inactivate than with a soil load. The soil load impacted on the effectiveness of AHP and this is evident when comparing results from wet load conditions, with or without soil load, at 1 minute contact time, which demonstrated that FCV with soil load required five times the concentration $(35000 \mathrm{ppm})$ to inactivate FCV compared to results without soil load which only required 7000 ppm. At 5 minute contact time, FCV with soil load required four times the AHP concentration at $7000 \mathrm{ppm}$ as opposed to without soil load which required only $1750 \mathrm{ppm}$ to inactivate the virus under wet load conditions; however, at a long contact time of 10 minutes, the AHP concentration gap was smaller, and the difference in concentration was only a two-fold dilution, from 1750 to 875 ppm. Under dry load conditions, the presence of a soil load influenced the effectiveness of Accel even more and made it more difficult to inactivate FCV, requiring $3500 \mathrm{pm}$ and long exposure times (10 minutes) to inactivate the virus with $4.8 \log _{10}$ reduction (Table 11 ).

Virox 5, by contrast, was unable to inactivate MNV-1, with or without soil load, at all contact times, with $<1.0 \log _{10}$ reduction after 10 minutes with soil load (Figure 11 and Figure 12).

Virox 5 was much more effective under wet load than dry load conditions, as demonstrated at the 5 and 10 minute contact times. Virox 5 was only able to inactivate FCV, with or without soil 
load, requiring an exposure time of 10 minutes to achieve a $>5.0 \log _{10}$ reduction (Figure 13 and Figure 14). AHP at a concentration of at least $3500 \mathrm{ppm}$ would be needed to inactivate FCV, with or without soil load, after 10 minutes; however, at shorter contact times of 5 minutes, a twofold higher concentration of $7000 \mathrm{ppm}$ or higher would be required to produce a $\sim 5.0 \log _{10}$ reduction and inactivate the virus.

Accel was unable to inactivate FCV with soil load after 1 minute under dry load conditions and this finding was not consistent with Omidbakhsh \& Sattar (2006); however it was noted that they had used Accel TB, a ready-to-use $0.5 \%$ AHP product, whereas in this study, a concentrated Accel product at $7.0 \%$ AHP was used alongside a ready-to-use Virox 5 product containing $0.5 \%$ AHP. It is possible that the three products contained different ingredients aside from AHP; however, the ingredients were not listed on either product and it would be difficult to assess whether each AHP product was actually different. The methodology of Omidbakhsh \& Sattar (2006) and this study also differed; glass Petri dishes were used instead of stainless steel discs in the current study, viral desiccation duration was 20 minutes as opposed to $60-90$ minutes in the current study and a Sephadex gel column was used instead of a neutralizer to remove cytotoxicity in the current study.

Overall, MNV-1 was more resistant towards AHP than FCV. Accel and Virox 5 were able to inactivate FCV but were generally ineffective against MNV-1. MNV-1 with soil load under dry load conditions required $35000 \mathrm{ppm}$ of AHP for inactivation at the longest exposure time of 10 minutes to achieve $>5.6 \log _{10}$ reduction, whereas FCV only required a concentration that was ten times as less concentrated under the same conditions (Table 11). It would be unrealistic and 
expensive to use AHP at such a high concentration of $35000 \mathrm{ppm}$ during norovirus outbreaks and during regular disinfection practices. Based on these results, AHP products (both concentrate and ready-to-use) may not be effective against human noroviruses.

\subsection{Efficacy of QUAT (CaviCide) and QUAT-Alcohol ( $\left.\mathrm{T}^{3} 6\right)$ against MNV-1 and}

\section{FCV, with and without Soil Load, under Wet and Dry Load Conditions}

QUATs have generally been shown to be ineffective against both MNV-1 and FCV (Belliot et al., 2008; Whitehead \& McCue, 2010) and results from this study are consistent with previous findings.

CaviCide, a ready-to-use QUAT containing $0.28 \%$ or 2800 ppm of diisobutylphenoxyethoxyethyl dimethyl benzyl ammonium chloride and $17.2 \%$ isopropanol, was ineffective against MNV-1, even in the absence of a soil load at all contact times (Figure 11). At 10 minutes, there was a 2.0 and $3.2 \log _{10}$ reduction under wet and dry load, respectively; however, this was not enough to inactivate the virus load to the desired level. CaviCide was not tested against MNV-1 with soil load due to its ineffectiveness against the virus in the absence of a soil load. Cytotoxicity was observed at the $10^{-1}$ dilution of the test carrier mixture, suggesting that the RAW 264.7 cells may be sensitive to alcohols such as isopropanol; however, CRFK cells did not exhibit any cytotoxic effects. Like MNV-1, CaviCide was also ineffective against FCV without soil load at all contact times (Figure 13). The highest $\log _{10}$ reduction was observed after 10 minutes with 3.6 and $3.3 \log _{10}$ reduction under wet and dry load, respectively; however, this was not enough to inactivate the virus. FCV with soil load was not tested because the selected QUAT product was already ineffective against the virus in the absence of a soil load. CaviCide 
was ineffective against both surrogate noroviruses and should not be used during norovirus outbreaks (Table 10 and Table 11).

A ready-to-use QUAT-alcohol product, $\mathrm{T}^{3} 6$, containing $0.20 \%$ or $2000 \mathrm{ppm}$ of benzalkonium chloride (QUAT), was also tested against both surrogate viruses (Figure 11 and Figure 13). This QUAT product differs from CaviCide in that it contains additional ingredients such as $0.28 \%$ $o$-phenylphenol and $0.01 \%$ chlorhexidine gluconate, but more importantly, $70 \%$ ethanol. QUATs in general have been reported to be ineffective against noroviruses but when other ingredients such as alcohol have been incorporated into the product, this study showed a different outcome. $\mathrm{T}^{3} 6$ was effective against MNV-1 without soil load after a 5 and 10 minute contact time with 6.9 and $6.2 \log _{10}$ reductions under wet and dry load conditions, respectively (Figure 11). Despite its effectiveness against MNV-1, the disinfectant was not effective against FCV in the absence of a soil load at all contact times at 10 minutes, with the highest $\log _{10}$ reductions at 2.4 and 2.9 under wet and dry load, respectively (Figure 13). Considering that QUATs are ineffective against non-enveloped viruses such as MNV-1 and FCV (Table 3), it is very likely that the high concentration of alcohol and not the QUAT compound was involved with the inactivation of MNV-1. However, it is possible that the other active ingredients such as $o$-phenylphenol and chlorhexidine gluconate may have played a role in the inactivation of MNV-1.

\subsection{Effect of Drying on Viral Titer}

The viral titers of MNV-1 and FCV (control group) were measured under the wet load condition and again after drying (dry load condition) after each experiment to observe the effects of 
desiccation at room temperature on the viral titer, with and without a soil load, when placed on stainless steel surfaces (Table 8). To preclude the possibility that some virus, especially in its dried state, may have continued to adhere to the stainless steel surface, vortexing was performed to dislodge as much inoculum from the disc as possible and to achieve elution. Viral titer loss was calculated by measuring the PFU/mL of the virus under wet load and dry load conditions and dividing the dry load titer by the wet load titer. This value expressed as a percentage established the fraction of virus remaining after drying. The differences in titer were also used to measure the log difference between wet and dry load conditions, and were calculated by subtracting the titer of virus under dry load conditions from those under wet load conditions. To determine if there were any significant differences between MNV-1 and FCV, with and without soil load, a two-sample t-test (assuming unequal variances) was used (Table B11 and Table B12). A t-test was also used to determine if there were any significant differences between each surrogate virus, with and without soil load (Table B13 and Table B14).

Comparing the percentage values of viral titer remaining, the t-test results showed that there were no significant differences between MNV-1, with and without soil load $(\mathrm{p}=0.47)$ (Table B13), and FCV, with and without soil load $(\mathrm{p}=0.44)$ (Table B14). The results showed that the presence of soil does not affect the viral titer remaining after it has undergone drying. There were significant differences when the percentage values of viral titer remaining of MNV-1 were compared to the values of FCV, both without soil load $(\mathrm{p}=0.00058)$ (Table B11). There were also significant differences when the percentage values of viral titer remaining of MNV-1 were compared to the values of FCV, both with soil load $(\mathrm{p}=0.0043)$ (Table B12). The results show 
that there are clear differences between the two surrogate viruses, with or without soil load, when they have undergone drying.

It was also observed that the PFU/mL and $\log _{10}$ reduction differed between the wet and dry load conditions for the controls (Table 8). Having the virus, with or without soil load, undergo drying on an environmental surface such as stainless steel for 60 to 90 minutes may have reduced the viral titer by as much as $\sim 0.5$ to $1 \log _{10}$ reduction. Drying MNV-1 resulted in a mean $\log _{10}$ reduction difference of 0.72 without soil load, and 0.77 with soil load, whereas drying of the FCV inoculum resulted in a mean $\log _{10}$ reduction difference of 0.39 without soil load and 0.44 with soil load.

Across both surrogate viruses, having the virus dried in the presence of a soil load resulted in a slightly higher mean $\log _{10}$ reduction, by as much as 0.06 for MNV-1 and 0.05 for FCV. Between 14 and $31 \%$ of the virus originating from the wet load viral titer remained after environmental desiccation for MNV-1 without soil load and between 10 to $25 \%$ remained with soil load, whereas 18 to $76 \%$ of the virus remained for FCV without soil load, and 17 to $55 \%$ remained with soil load. On average, $20 \%$ of the wet load viral titer remained for MNV-1 without soil load after drying and $18 \%$ with soil load, whereas $43 \%$ remained for FCV without soil load and $38 \%$ remained with soil load, suggesting that drying the virus in the presence of the soil load, had reduced the virus titer by as much as $2 \%$ for MNV-1 and $5 \%$ for FCV.

The effect of drying on the viral titer differed between each surrogate virus, with MNV-1 showing more viral inactivation than FCV. In the absence of a soil load, the difference between 
the mean viral titer remaining of MNV-1 and FCV was $23 \%$, indicating that drying had a more negative effect on MNV-1 than FCV. With a soil load, the difference in mean viral titer remaining between both surrogate viruses was at $20 \%$. This suggests that FCV was more tolerant of drying conditions than MNV-1, and that more FCV survived after drying. It has been reported that in its dried state, FCV can survive at $4{ }^{\circ} \mathrm{C}$ for between 21 to 28 days (Doultree et al., 1999). At room temperature and in a fecal matrix, MNV-1 under wet load conditions was more stable than FCV on stainless steel surfaces over a 7 day period; however, dried MNV-1 was less stable compared to FCV (Cannon et al., 2006). Lamhoujeb et al. (2009) had found that the recovery of human norovirus in fecal matter on stainless steel after 30 minutes was $14.2 \%$, and up to $80 \%$ of human norovirus surface dried in a laminar flow hood were undetectable after 30 minutes of drying. The results from this study are in agreement with results from previous studies which suggest that MNV-1 is less resistant to environmental desiccation than FCV and that recovery of the virus after desiccation may be significantly less.

Despite the reduction in viral titer after drying, especially in the presence of a soil load, these findings do not necessarily indicate that the presence of any soil load hinders the overall survival of noroviruses. In fact, the presence of organic matter or a protein soil load has been shown to reduce the efficacy of disinfectants (McDonnell \& Russell, 1999). For a disinfectant or biocide to be effective, the concentration must be maintained, but its efficacy can be hindered by organic matter (Maillard, 2001). Organic matter, such as blood, serum, soil, feces and other organic materials, can reduce the effective concentration of the disinfectant or can protect viral particles from the virucidal effects of the biocide (Gélinas \& Goulet, 1983; Maillard, 2001). Gélinas \& Goulet (1983) reported that glutaraldehyde was most resistant to neutralization by organic matter 
because the neutralization sites of the organic matter become rapidly saturated by absorbing the disinfectant molecules. Chlorhexidine acetate and amphoteric surfactants are moderately affected by organic matter whereas QUATs have a low tolerance to organic matter (consisting of protein) due to the adsorption pattern of QUAT compounds and the constant absorbing of the disinfectant molecules by organic matter (Gélinas \& Goulet, 1983). Disinfectants with a very low tolerance to the presence of organic matter include anionic acids, sodium hypochlorite and iodophor (Gélinas \& Goulet, 1983). The type and chemical composition of organic matter present may have a greater effect on its interfering behaviour towards disinfectants (Gélinas \& Goulet, 1983). As the protein content increases, interference towards disinfectants also increases (Gélinas \& Goulet, 1983).

The findings of this study show that the presence of soil consisting of tryptone, BSA and bovine mucin equivalent to a $5 \%$ solution of FBS (or $2 \mathrm{~g}$ of total protein/L) interfered with the efficacy of the disinfectants, namely, sodium hypochlorite, Accel and Virox 5, for both MNV-1 and FCV. The differences between the $\log _{10}$ reduction of wet load conditions with soil load versus without soil load, and dry load conditions with soil load versus without soil load, varied between each disinfectant. For sodium hypochlorite, MNV-1 in the presence of a soil load under wet load conditions was not affected as much as compared to the $\log _{10}$ reduction of MNV-1 without soil load (Table 10). A surprising finding was that the results showed an opposite effect, where the presence of soil under wet conditions showed a higher $\log _{10}$ reduction for some values, especially at lower chlorine concentrations (between 338 and $1350 \mathrm{ppm}$ ) where virus was not entirely inactivated at all contact times, than without soil load. For example, at $675 \mathrm{ppm}$ of available chlorine at the 1 and 5 minute contact times under wet load conditions, the $\log _{10}$ 
reduction for MNV-1 without soil load was 1.91 and 4.13, respectively, whereas with the soil load, it was 4.03 and $5.62 \log _{10}$ reduction, indicating that the presence of a soil load may have allowed more virus to become inactivated, thus leading to a higher $\log _{10}$ reduction. This may mean that the presence of a soil load with MNV-1 was easier to inactivate than MNV-1 without a soil load. It is possible that the concentration of total protein in the soil load was low enough to not have a noticeable effect on the effectiveness of the disinfectant, and that higher protein loads may be required to see a significant difference. Under dry load conditions, MNV-1 with the presence of a soil load appeared to reduce the efficacy of sodium hypochlorite, especially at lower chlorine concentrations of 675 ppm and longer contact times; however, like the results from the wet load conditions, the absence of a soil load appeared to reduce the efficacy of sodium hypochlorite and $\operatorname{lower} \log _{10}$ reductions are observed than with soil load. It is not understood why the presence of the soil load involving MNV-1 does not appear to reduce the efficacy of sodium hypochlorite but rather, increase viral inactivation as shown by its higher $\log _{10}$ reduction. AHP-based disinfectants on the other hand showed a different outcome. The presence of a soil load decreased the efficacy of both Accel and Virox 5 when inactivating MNV-1. Higher $\log _{10}$ reductions were observed consistently under wet and dry load conditions for MNV-1 without soil load versus with a soil load.

For FCV, the presence of a soil load appeared to hinder the efficacy of sodium hypochlorite under wet load conditions at all contact times between 1350 to 5400 ppm hypochlorite; however, for concentrations of 338 to $675 \mathrm{ppm}$, an opposite effect was observed, where there was a higher $\log _{10}$ reduction for FCV with soil load at the 5 minute contact time compared to without soil load (Table 11). Under dry conditions, the presence of a soil load resulted in much lower $\log _{10}$ 
reductions than without soil load, suggesting that having the virus on a soil load dried on a surface is much more difficult to inactivate, especially at high chlorine concentrations and short contact times. The efficacy of Accel and Virox 5 against FCV were also consistently reduced under wet and dry load conditions throughout all contact times when a soil load was present. Overall, the results suggest that the presence of a soil load can adversely affect the effectiveness of the disinfectant, in particular AHP-based disinfectants and sodium hypochlorite, but can also be dependent on the type of virus present, as observed by results involving MNV-1 and sodium hypochlorite.

\subsection{Cytotoxicity and Virus Infectivity Control Test}

Disinfectants and their neutralizers may be highly toxic for cell lines so a cytotoxicity test and an interference/virus infectivity test was conducted at the beginning of the study to make sure the disinfectant and neutralizer did not affect the cell line or virus, or both. Another possible option was to use the gel filtration method to remove the disinfectant from the virus after contact in order to reduce chemical cytotoxicity of the cell culture; however, this method may not necessarily eliminate disinfectant cytotoxicity (Aidaros \& Gaudin, 1983; ASTM International, 1998; Sattar et al., 2003).

The following test formulations and reagents were selected for the cytotoxicity control test: Accel (35000 ppm), Accel (17500 ppm), CaviCide, $\mathrm{T}^{3} 6$, sodium hypochlorite (5400 ppm), Virox 5, sodium thiosulphate neutralizer, letheen broth neutralizer, soil load mixture and EBSS (the control). Results (PFU) in triplicate were analysed using single factor ANOVA, with $\mathrm{p}<0.05$ being statistically significant. For treatments that were significantly different, Tukey’s 
Honestly Significant Difference (HSD) Test was used to determine which treatments were significantly different.

\subsubsection{Cytotoxicity Observations for RAW 264.7 and CRFK Cells}

Cytotoxicity, documented by microscopy, was not observed when monolayers of RAW 264.7

macrophage mouse cells were exposed to a $10^{-1}$ serial dilution of test solution containing Accel at $17500 \mathrm{ppm}$, sodium hypochlorite at $5400 \mathrm{ppm}$ and Virox 5 at $5000 \mathrm{ppm}$, both with and without neutralizer, to arrest the activity of the disinfectant (Table 6). Cytotoxicity was also not observed from solutions such as sodium thiosulphate neutralizer, letheen broth neutralizer, soil load mixture and EBSS. The neutralizers did not have any cytotoxic effects on the macrophage mouse cell culture. Some cytotoxicity $(<10 \%)$ was observed for the Accel at $35000 \mathrm{ppm}$ without the addition of neutralizer, however, this was not substantial, as over $90 \%$ of the monolayer was able to absorb the neutral red dye. There was no visible cytotoxicity for Accel at $35000 \mathrm{ppm}$ with neutralizer, which indicates that the neutralizer was able to arrest the activity of the disinfectant. Cytotoxicity was observed for CaviCide (with and without neutralizer) at the $10^{-1}$ dilution when the cell culture was examined with the microscope but no cytotoxic effects were observed at the $10^{-2}$ dilution. At the $10^{-1}$ dilution for CaviCide, the entire monolayer of RAW 264.7 cells was unable to absorb the neutral red dye, indicating that all of the cells were dead which may have been due to sensitivity to the isopropanol in the CaviCide. Cytotoxicity was observed, with and without neutralizer, during exposure to the $\mathrm{T}^{3} 6$ disinfectant at the $10^{-1}$ and $10^{-2}$ dilutions; however, cytotoxicity was not observed at the $10^{-3}$ dilution. When $\mathrm{T}^{3} 6$ was tested (at $20 \mu \mathrm{L}$ of T $\mathrm{T}^{3} 6$ with $980 \mu \mathrm{L}$ of letheen broth/EBSS), cytotoxicity was observed at the $10^{-1}$ dilution, however cytotoxicity was not visible at the $10^{-2}$ dilution. 
Because cytotoxicity was observed when RAW 264.7 host cells were exposed to CaviCide and $\mathrm{T}^{3} 6$ at $10^{-1}$ dilution, experiments had to be conducted and plaques only documented at the $10^{-2}$ dilution. If no viral plaques were observed at $10^{-2}$ dilution, it is still possible that $\leq 100$ viable PFU may have been present. Both QUAT disinfectants contain alcohol, with CaviCide containing $17.2 \%$ isopropanol and $\mathrm{T}^{3} 6$ containing $70 \%$ ethanol. It is possible that the alcohol present in the QUAT disinfectants may have caused cytotoxicity in the host cells.

Cytotoxicity was not observed when monolayers of CRFK cells were exposed to a $10^{-1}$ serial dilution containing sodium hypochlorite (5400 ppm), CaviCide and Virox 5, with and without neutralizer, to arrest the activity of the disinfectant (Table 6). Cytotoxicity was not observed, with and without neutralizer, during exposure to the $\mathrm{T}^{3} 6$ disinfectant at the $10^{-1}$ and $10^{-2}$ dilution. When $\mathrm{T}^{3} 6$ was tested, with and without neutralizer, cytotoxicity was not observed at the $10^{-1}$ dilution (originating from the test solution consisting of $20 \mu \mathrm{L}$ of $\mathrm{T}^{3} 6$ and $980 \mu \mathrm{L}$ of letheen broth/EBSS). Cytotoxicity was also not observed from selected reagents such as sodium thiosulphate neutralizer, letheen broth neutralizer, soil load mixture and EBSS. Cytotoxicity was observed when the cell culture was exposed to Accel (35000 ppm), with and without neutralizer. Between 80 to $90 \%$ of the monolayer of cells showed visible cytotoxic effects when exposed to Accel $(35000 \mathrm{ppm})$ without neutralizer and were not able to take in the neutral red dye from the agarose overlay mixture. Exposure to Accel (35000 ppm) with neutralizer caused minimal visible cytotoxicity of less than $10 \%$ and the majority of cells were able to absorb the neutral red dye from the agarose overlay mixture, suggesting that the presence of letheen broth neutralizer substantially decreased cytotoxicity in the cell culture caused by the AHP disinfectant. Exposure 
to Accel (17500 ppm) with neutralizer did not result in any visible cytotoxicity in the CFRK cells.

\subsubsection{Virus Infectivity of MNV-1 towards RAW 264.7 Cells}

The effect of the soil load, neutralizer (sodium thiosulphate and letheen broth), disinfectant with neutralizer and disinfectant without neutralizer were tested with statistical analysis (ANOVA single factor) and Tukey's HSD Test to determine whether there were any virus infectivity issues of MNV-1 towards the RAW 264.7 cell line (Table B1).

The presence of the soil load mixture did not adversely affect the ability of MNV-1 to infect or replicate in RAW 264.7 macrophage mouse cells $(p=0.22)$ and showed no significant difference when compared to the control (EBSS). Both neutralizers, sodium thiosulphate and letheen broth, showed no significant difference $(\mathrm{p}=0.80)($ Table B1).

Disinfectants with neutralizer, disinfectants without neutralizer and the control (EBSS) were compared with each other and analysed using single factor ANOVA to determine whether the disinfectant (alone) or the combination of disinfectant and neutralizer interfered with the virus infectivity or replication of MNV-1 in host cells (Table B1). If there were significant differences $(\mathrm{p}<0.05)$, Tukey's HSD Test was performed to determine which treatments were significantly different (Table B9). There were no significant differences between the control, disinfectant with neutralizer and disinfectant without neutralizer: sodium hypochlorite $(5400 \mathrm{ppm})(\mathrm{p}=0.97)$, CaviCide $(p=0.33)$, Virox $5(p=0.77)$ and $T^{3} 6(20 \mu L)(p=0.20)$, suggesting that the disinfectant, with or without neutralizer, had no effect on the challenge virus nor the host cell. 
However, there was a significant difference for Accel $(35000 \mathrm{ppm})(\mathrm{p}=0.00048)$ and Accel $(17500 \mathrm{ppm})(\mathrm{p}=0.00041)$ at the $10^{-1}$ dilution. Because cytotoxicity was observed for Accel (35000 ppm) without neutralizer, a comparison between the disinfectant with neutralizer, disinfectant without neutralizer and the control could not be calculated and the Tukey's HSD Test could not be used.

Although cytotoxicity of RAW 264.7 cells was not observed for Accel at 35000 and 17500 ppm with neutralizer at $10^{-1}$ dilution (Table 6), there were interference/virus infectivity issues, most notably a reduction in the viral plaque average at $35000 \mathrm{ppm}$ AHP with a mean PFU value of 22.3 for the control compared to $4.7 \mathrm{PFU}$ for the disinfectant and neutralizer combination, a difference of 17.7 PFU; and for 17500 ppm AHP, a mean PFU value of 13.7 for the disinfectant with neutralizer, a difference of 8.7 PFU when compared to the control mean PFU, suggesting that the ability of MNV-1 to infect and replicate in RAW 264.7 cells was affected (Table 7 and Table B1). An increase in the neutralizer strength to arrest the activity of highly concentrated AHP would have led to cytotoxicity in the RAW 264.7 host cells so it would have been impossible to increase its strength. Because of the reduction in MNV-1 infectivity of RAW 264.7 macrophage mouse cells, the PFU average could be higher than previously observed, possibly by as much as 17.7 PFU for $35000 \mathrm{ppm}$ AHP or $8.7 \mathrm{PFU}$ for $17500 \mathrm{ppm}$ AHP. The presence of Accel at extremely high concentrations (e.g., 35000 ppm) with neutralizer may have reduced the ability of MNV-1 to infect RAW 264.7 host cells or it may have compromised the ability of the host cells to be infected by the virus, thereby interfering with the estimation of the virucidal activity of the disinfectant. Another explanation may be the strength of the neutralizer. It is possible that the neutralizer may not have been concentrated enough to fully arrest the 
activity of the disinfectant and there may have been some AHP residue present to interfere with the host cell, virus, or both; however, the strength of the neutralizer was adequate for FCV, suggesting that the neutralizer may have been strong enough to arrest the activity of the Accel product. Lower concentrations of AHP were not effective against the virus and did not have any impact on the cell culture.

\subsubsection{Virus Infectivity of FCV towards CRFK Cells}

The effect of the soil load, neutralizer (sodium thiosulphate and letheen broth), disinfectant with neutralizer and disinfectant without neutralizer were tested with statistical analysis (ANOVA single factor) and Tukey's HSD Test to determine whether there were any virus infectivity issues of FCV towards the CRFK cell line (Table B2).

The presence of the soil load mixture did not adversely affect the ability of FCV to infect or replicate in CRFK cells $(\mathrm{p}=0.52)$ (Table B2). Both neutralizers, sodium thiosulphate and letheen broth, were compared to the control using single factor ANOVA and showed no significant difference $(\mathrm{p}=0.67)$.

Disinfectants with neutralizer, disinfectants without neutralizer and the control (EBSS) were compared with each other and analysed using single factor ANOVA to determine whether the disinfectant (alone) or the combination of disinfectant and neutralizer interfered with the virus infectivity or replication of FCV in CRFK cells (Table B2). If there were significant differences ( $\mathrm{p}<0.05)$, Tukey's HSD Test was performed to determine which treatments were significantly different (Table B10). There were no significant differences between the control, disinfectant 
with neutralizer and disinfectant without neutralizer for CaviCide $(\mathrm{p}=0.20)$. However, there were significant differences between the following: sodium hypochlorite $(5400 \mathrm{ppm})(\mathrm{p}=0.018)$, Virox $5(\mathrm{p}=0.0021), \mathrm{T}^{3} 6(20 \mu \mathrm{L}$ volume $)(\mathrm{p}=0.021)$ and $\mathrm{T}^{3} 6(50 \mu \mathrm{L}$ volume $)(\mathrm{p}=0.00071)$ at

the $10^{-1}$ dilution. Lower mean PFU values were observed for sodium hypochlorite (5400 ppm) with neutralizer at 15.0 PFU compared to the control which had a mean PFU of 19.7. Higher mean PFU values were observed for Virox 5, $\mathrm{T}^{3} 6(20 \mu \mathrm{L})$ and $\mathrm{T}^{3} 6(50 \mu \mathrm{L})$, with 31.0, 29.7 and 38.0 PFU, respectively, compared to the control which had a mean PFU of 19.7, resulting in a difference of 11.3 PFU for Virox 5 and 10.0 PFU for $\mathrm{T}^{3} 6$ (Table B2). Although the cause for the factors involved with the increase in PFU could not be determined, the results had suggested that the presence of the disinfectant with neutralizer may have enhanced the ability of FCV to infect or replicate in CRFK cells.

Because cytotoxicity was observed for Accel (35000 ppm) without neutralizer, a comparison between the disinfectant with neutralizer, disinfectant without neutralizer and the control could not be calculated and the Tukey's HSD Test could not be used. There was no significant difference between Accel (35000 ppm) $(\mathrm{p}=0.35)$ with neutralizer and the control suggesting that presence of the disinfectant and neutralizer mixture did not interfere with the ability of FCV to infect or replicate in the host cells.

\subsection{Challenges}

One unanticipated finding of this study was that MNV-1 was more sensitive, susceptible and more easily inactivated by disinfectants containing alcohol than FCV. FCV was not inactivated by disinfectants containing alcohol, in particular $\mathrm{T}^{3} 6$. One major challenge is the inability to 
neutralize and arrest the cytotoxicity of alcohol. Instead, the alcohol from the $\mathrm{T}^{3} 6$ needed to be diluted during experimental trials to minimize the activity of the alcohol against the surrogate viruses. Another issue was the sensitivity of RAW 264.7 macrophage mouse cells during alcohol exposure. This sensitivity led to cytotoxic effects being observed at the $10^{-1}$ serial dilution range and difficulty in observing viral plaques at that dilution. The $10^{-2}$ serial dilution originating from the test solution ( $50 \mu \mathrm{L}$ of $\mathrm{T}^{3} 6$ that was applied to $10 \mu \mathrm{L}$ of virus on stainless steel discs) was too cytotoxic for the RAW 264.7 macrophage mouse cells and as a result, the amount of disinfectant that was applied to the surrogate virus had to be lowered to $20 \mu \mathrm{L}$ to minimize the cytotoxic effects. Letheen broth neutralizer was applied at $980 \mu \mathrm{L}$ instead of $950 \mu \mathrm{L}$. Even with the slight modification in the methodology, the $10^{-1}$ serial dilution still contained enough alcohol to kill the monolayer of cells attached to the surface of 6-well plates, thus preventing neutral red from being absorbed and making it difficult for viral infection to occur. As a result, MNV-1 plaques had to be counted at the $10^{-2}$ serial dilution to neutralize the alcohol. Because of this limitation, inactivation of MNV-1 observed at the $10^{-2}$ dilution may not be an accurate reflection of the effectiveness of $\mathrm{T}^{3} 6$ against MNV-1, and may still contain infectious virus at lower dilutions (e.g., $10^{-1}$ or $10^{0}$ dilution) of up to $\leq 100 \mathrm{PFU}$.

Another challenge of this study was the inability to determine an accurate number of MNV-1 survivors when exposed to Accel at 35000 and $17500 \mathrm{ppm}$. Due to the reduction in virus infectivity for MNV-1, it was not possible to determine exactly how much virus had survived after a 10 minute exposure. As such, the $\log _{10}$ reduction values should be viewed as an estimate. Another challenge was the alcohol sensitivities exhibited by MNV-1 towards CaviCide and $\mathrm{T}^{3} 6$. Due to the cytotoxicity displayed by RAW 264.7 macrophage mouse cells, it was not possible to 
determine how much virus had survived at the $10^{-1}$ dilution range. Because of this limitation, there could be up to $\leq 100 \mathrm{PFU}$ present after exposure to the following disinfectants and the $\log _{10}$ reduction values should be viewed as an estimate. Cytotoxicity was also observed by Park et al. (2010) in RAW 264.7 and CRFK cells when exposed to alcohol-based hand sanitizers (such as ethanol and isopropanol), where an additional 10-fold dilution was needed to eliminate cytotoxicity completely.

Cytotoxic effects were not observed at the $10^{-1}$ serial dilution range for CRFK cells (when $50 \mu \mathrm{L}$ of $\mathrm{T}^{3} 6$ was used) and it does not appear FCV is sensitive or susceptible to alcohol exposure at all; however, for consistency, a volume of $20 \mu \mathrm{L}$ of $\mathrm{T}^{3} 6$ was also used on FCV. It is possible that RAW 264.7 macrophage mouse cells and MNV-1 may be more sensitive towards alcohols than FCV and CRFK cells. Observations from isopropanol exposure (from the CaviCide disinfectant) towards RAW 264.7 macrophage mouse cells appear to support this. These findings are consistent with a comparative study involving surrogate viruses, MNV-1 and FCV, and ethanolbased handrubs, which found MNV-1 to be more readily inactivated by alcohol handrubs in short contact times than FCV (Park et al., 2010; Sattar et al., 2011). Park et al. (2010) suggested that the inactivation pattern of MNV-1 is similar to hydrophilic non-enveloped viruses such as poliovirus and Coxsackie B1 virus, which are susceptible to ethanol and isopropanol at $70 \%$ concentration or higher. Viruses which are related and are in the same family can show some differences in sensitivities towards disinfectants (Maillard, 2001). One important limitation to disclose is that viral plaques were counted (as PFU/mL) at the $10^{-1}$ serial dilution range (with the exception of MNV-1 with $\mathrm{T}^{3} 6$ where plaques were counted at $10^{-2}$ dilution) meaning if there 
were zero plaques observed at the $10^{-1}$ dilution, it may be possible to have up to $\leq 10 \mathrm{PFU}$ survivors at the $10^{0}$ dilution.

One of the reasons why the $\log _{10}$ reduction of FCV was on average lower than MNV-1 could be attributed to the viral titer of the stock at the start of the experiment trials. When MNV-1 was inactivated by sodium hypochlorite, the results were on average $>6 \log _{10}$ reduction compared to FCV results which showed a $\sim 5$ to $5.5 \log _{10}$ reduction. Had the viral titer of the FCV stock been at least $1 \log$ higher, it may have been possible to see more viral inactivation of $>6 \log _{10}$ reduction at the same chlorine concentrations and contact times already achieved with MNV-1. Because all three contact times could not be performed in the same experiment and at the same time (with the exception of RTU disinfectants), a new and different virus titer stock was used for each experiment. As such, the virus titer, with or without soil load, would be slightly different for each experiment; however, the virus inoculum range was similar. There were slight variations in the virus titer stock for each experiment and contact time, and it may be difficult to compare each result with each other because the results are not absolute values.

The $\mathrm{pH}$ of the disinfectant can play a significant role on the stability of MNV-1 and FCV (Cannon et al., 2006; Whitehead \& McCue, 2010). FCV was inactivated when exposed to disinfectants at $\mathrm{pH}<3$ and $>9$, whereas MNV-1 was inactivated at $\mathrm{pH}<2$ and $>10$ (Cannon et al., 2006; Whitehead \& McCue, 2010). Human noroviruses can survive in gastric pH levels (Cannon et al., 2006). pH may play an important role in the replication of non-enveloped viruses. Kreutz \& Seal (1995) reported that FCV infection of CRFK cells involved a low pHdependent viral uncoating step, although it was unclear how this mechanism worked. The 
efficacy of chlorine-based disinfectants is dependent on the concentration and $\mathrm{pH}$ but their efficacy may be reduced in the presence of organic matter (McDonnell \& Russell, 1999). QUATs on the other hand are generally more effective in alkaline $\mathrm{pH}$ ranges (Marriott \& Gravani, 2006). MNV-1, which has a capsid structure, genomic organization and replication cycle similar to human norovirus, was shown to be sensitive towards alcohol; however, it is unknown at the time if human norovirus is also sensitive and less tolerant towards alcohol-based disinfectants.

It was more difficult to inactivate either surrogate virus when the virus underwent more extreme conditions such as environmental desiccation (dry load conditions) in the presence of a soil load because the efficacy of disinfectants may be reduced in the presence of organic matter such as a protein soil load. When selecting which disinfectants, concentrations and contact times that produced the best inactivation of MNV-1 and FCV, it is advisable to select one that can inactivate either surrogate virus under more realistic settings (e.g., dried virus containing a soil load on an environmental hard surface) and shorter contact times of 1 or 5 minutes, as longer contact times of 10 minutes may be too long and unrealistic.

\subsection{Mechanism of Virucidal Activity and Overall Assessment of Most Suitable Surrogate Virus for Human Norovirus}

The virucidal activity of the selected disinfectants may vary, depending on the presence or absence of a lipid envelope and the size of the virus (Maillard, 2001). Enveloped viruses which possess a lipid envelope tend to be more susceptible to lipophilic-type disinfectants such as 2-phenylphenol, QUATs, chlorhexidine, isopropanol, ether and chloroform (McDonnell \& 
Russell, 1999). Non-enveloped viruses on the other hand are considered more resistant to biocides than enveloped viruses (Maillard, 2001). The concentration, contact time, pH, organic load and temperature also play an important role in the activity of biocides (Maillard, 2001). Virucides may have multiple target sites on the viral particles leading to damage and loss of viral infectivity, and an increase in the concentration of the disinfectant or biocide may increase the structural damage of the viral particle (Maillard, 2001). The mechanisms behind the virucidal activity of biocides are generally less documented, and studies on microorganisms including bacteria, spores and yeast are often used to help explain the virucidal activity of biocides (Maillard, 2001). The major differences between viruses and other microorganisms are that viruses are smaller, have a simple structure and do not show any metabolic activity, restricting target sites for virucides (Maillard, 2001). It is speculated that the mechanisms mostly involve altering the viral capsid structure but may not always damage viral nucleic acid (Maillard, 2001). Biocides such as glutaraldehyde, hypochlorite, ethylene oxide and hydrogen peroxide are known to react strongly with amino or sulfhydryl groups and may have virucidal activity (McDonnell \& Russell, 1999). Biocides can react strongly with proteins although their activity may be limited to capsid proteins and not to the viral nucleic acid (Maillard, 2001). Even so, viral markers, such as antigenic structure and DNA polymerase, which get altered, and structural damage to the viral capsid does not always result in the loss of viral infectivity (Maillard, 2001). Of the few mechanisms identified, the formation of viral aggregates may be attributed to viral resistance to disinfectants (Maillard, 2001). Certain disinfectants such as iodine, QUATs, alcohols and phenolic compounds may have their virucidal activities limited to only the capsid and not the viral genome, whereas acids may lead to a change in the capsid conformation and result in a loss of capsid integrity (Maillard, 2001). For viral inactivation to occur, its nucleic acid must be 
rendered non-infectious. This can occur by either permanently immobilizing the viral particle on surfaces, or by blocking or destroying the host cell receptors; however, damaging the viral capsid and nucleic acid using chlorine-releasing agents, oxidizing agents and ozone may also work (Maillard, 2001; McDonnell \& Russell, 1999). Overall, there are a number of possible virusbiocide interactions taking place during disinfection leading to damage of viral markers, envelope (for enveloped viruses), capsid structure, nucleic acid or genome (Maillard, 2001).

Sodium hypochlorite, AHP, QUAT disinfectant products were examined. Certain products were more effective against one surrogate virus than the other surrogate virus, with the exception of sodium hypochlorite which was effective against both surrogate viruses. Although AHP was effective against both surrogate viruses, this was only possible by raising the concentration of AHP up to $35000 \mathrm{ppm}$ in order to inactivate MNV-1 after a 10 minute contact time, as opposed to FCV which did not require such a high concentration in order to be inactivated. MNV-1 was resistant towards certain disinfectants compared to FCV, and vice versa. It is unknown why MNV-1 and FCV responded differently to each disinfectant.

Using the performance criteria of a $4 \log _{10}$ reduction in viral titer, Domestic Miraclean Bleach (sodium hypochlorite), Accel and $\mathrm{T}^{3} 6$ were effective against MNV-1, while Domestic Miraclean Bleach, Accel and Virox 5 were effective against FCV. In the absence of a pre-rinsing and/or pre-cleaning step prior to disinfection as listed on the manufacturer's recommendations on the product's labels, sodium hypochlorite at a concentration of at least $2700 \mathrm{ppm}$ after a 5 minute contact time proved to be most effective against both surrogate viruses with $\sim 5 \log _{10}$ reduction for FCV and >5.5 $\log _{10}$ reduction for MNV-1. Accel at 35000 ppm inactivated both viruses; 
however, the very high concentration does not appear to be suitable or practical for use in disinfecting environmental hard surfaces. In addition, there are virus infectivity issues surrounding RAW 264.7 macrophage mouse cells from exposure to the disinfectant (with neutralizer) such as a reduction in the PFU and it would be difficult to determine how much virus had survived.

Based on these findings, there is no clear answer to determine whether MNV-1 or FCV is the most suitable surrogate virus to study the stability and tolerance of human norovirus. MNV-1 showed greater resistance to QUATs and AHP-based disinfectants than FCV even though FCV showed slightly greater resistance to sodium hypochlorite than MNV-1. Human norovirus and MNV-1 both share features such as size, shape and buoyant density characteristics, and belong in the same Norovirus genus (Hsu et al., 2007; Karst, 2010). Although MNV-1 and human noroviruses are very distinct strains, MNV-1 remains the closest surrogate virus to human norovirus than FCV (Hsu et al., 2007).

In this study, MNV-1 was more sensitive towards disinfectants containing alcohol than FCV. If human noroviruses were more resistant towards alcohol-based disinfectants and handrubs containing a minimal concentration of $70 \%$ alcohol, it might be possible to see similar results which demonstrated a 2.42 to $2.90 \log _{10}$ reduction in FCV titer after 10 minutes (without soil load) using the $\mathrm{T}^{3} 6$ disinfectant. Despite this possibility, human norovirus, like MNV-1, may be just as sensitive and less tolerant towards alcohol-based disinfectants. 
Because both surrogate viruses demonstrated differences in susceptibility towards alcohols, it may be important to continue using both surrogate viruses for in vitro testing such as plaque assay, to provide a more reliable estimate of the effectiveness of disinfectants against human norovirus. 
A

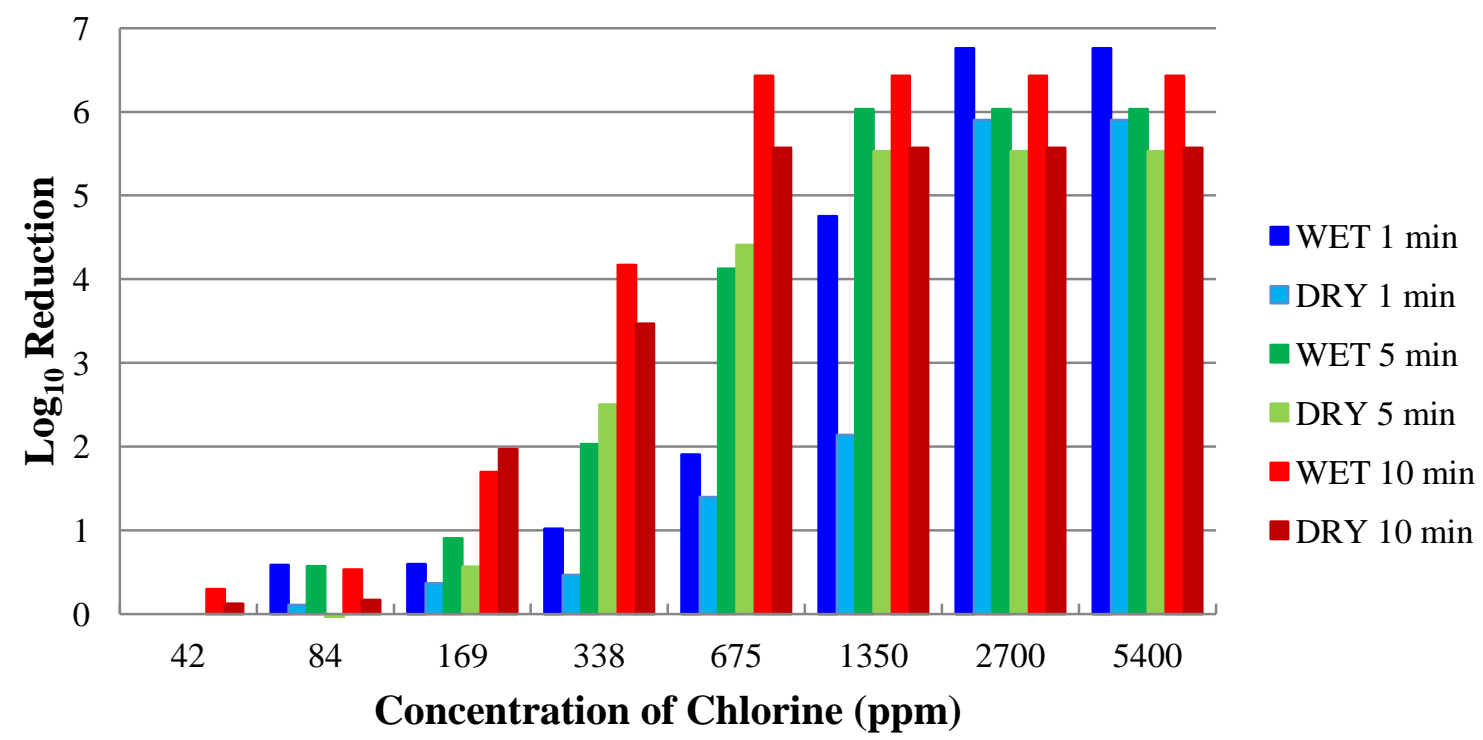

B

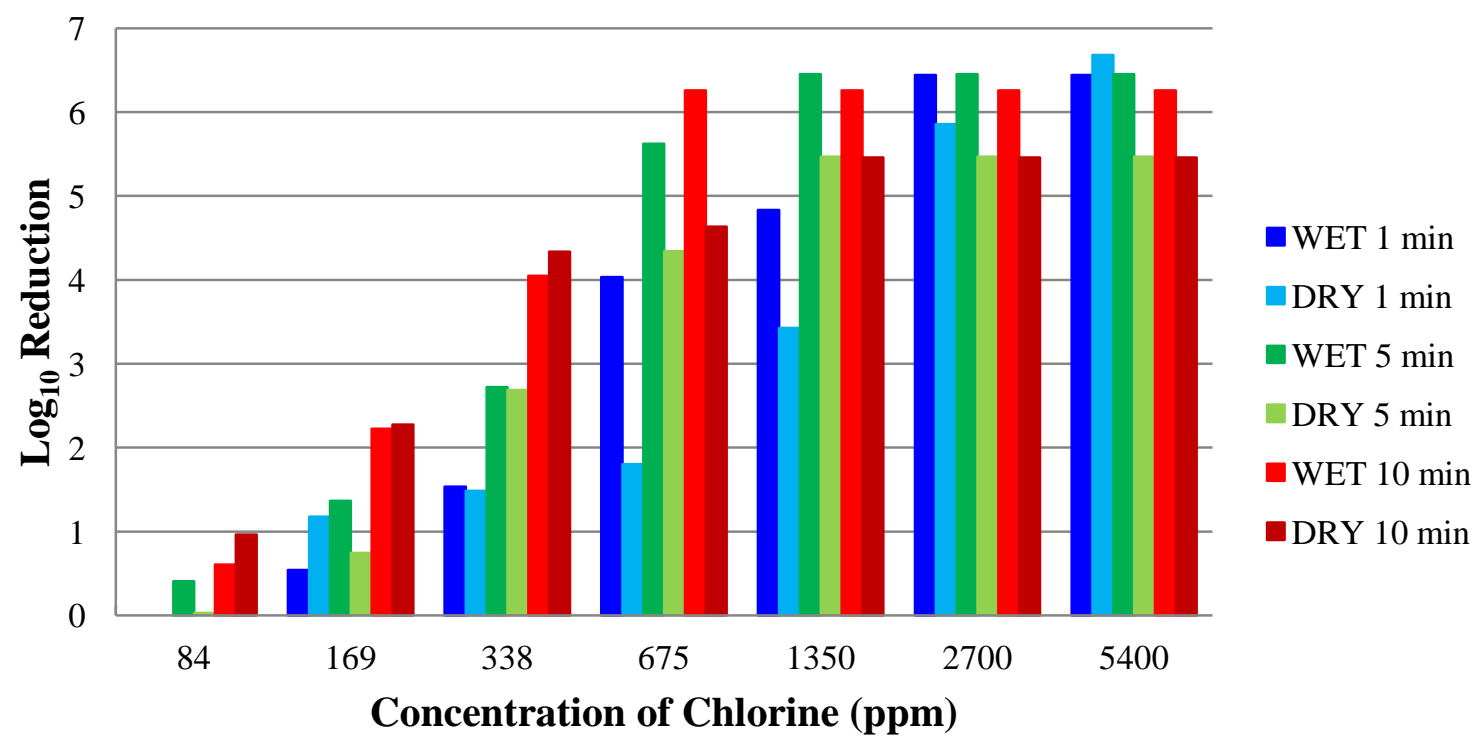

Figure 7. Effectiveness of sodium hypochlorite against MNV-1, (A) without soil load and (B) with soil load, at 1, 5 and 10 minute contact times, under wet and dry load conditions. WET refers to wet load conditions and DRY refers to dry load conditions. 
A

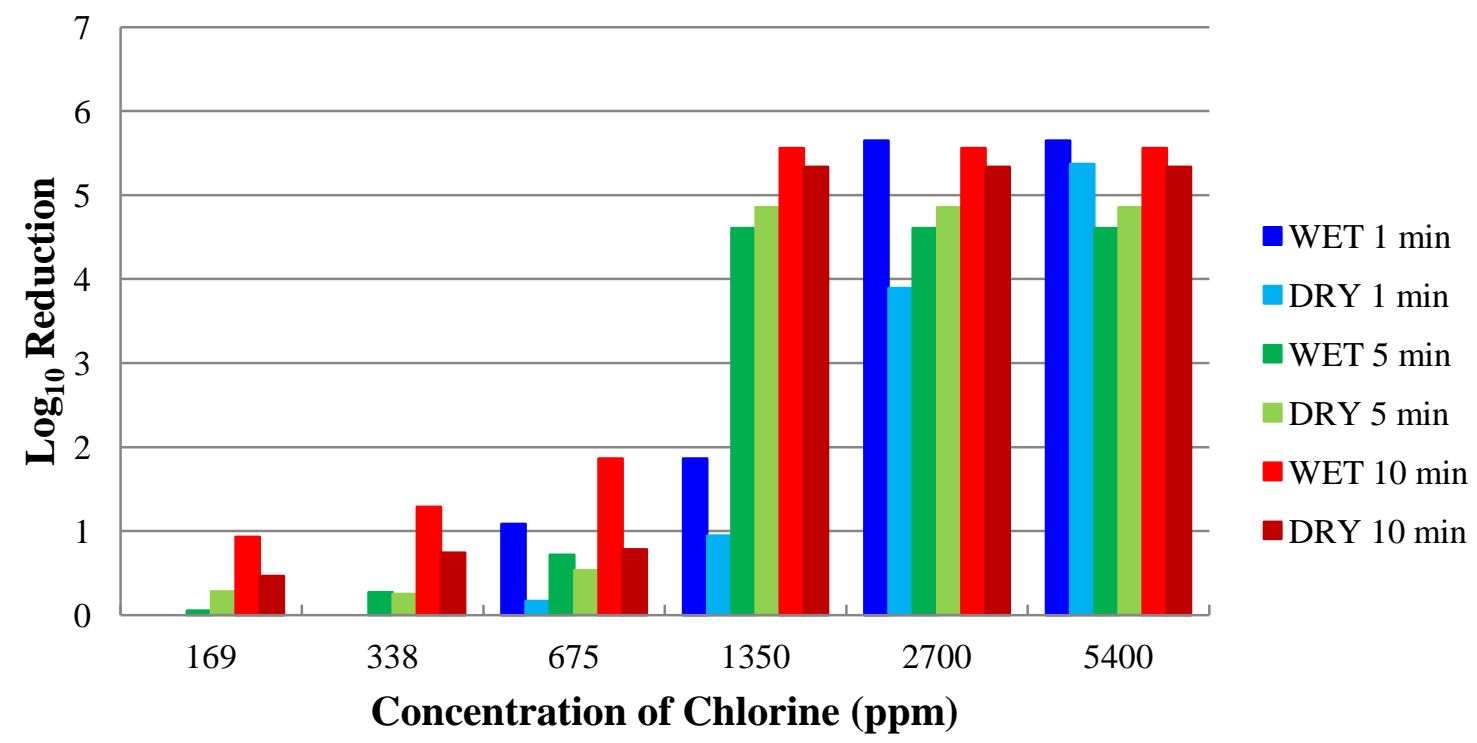

B

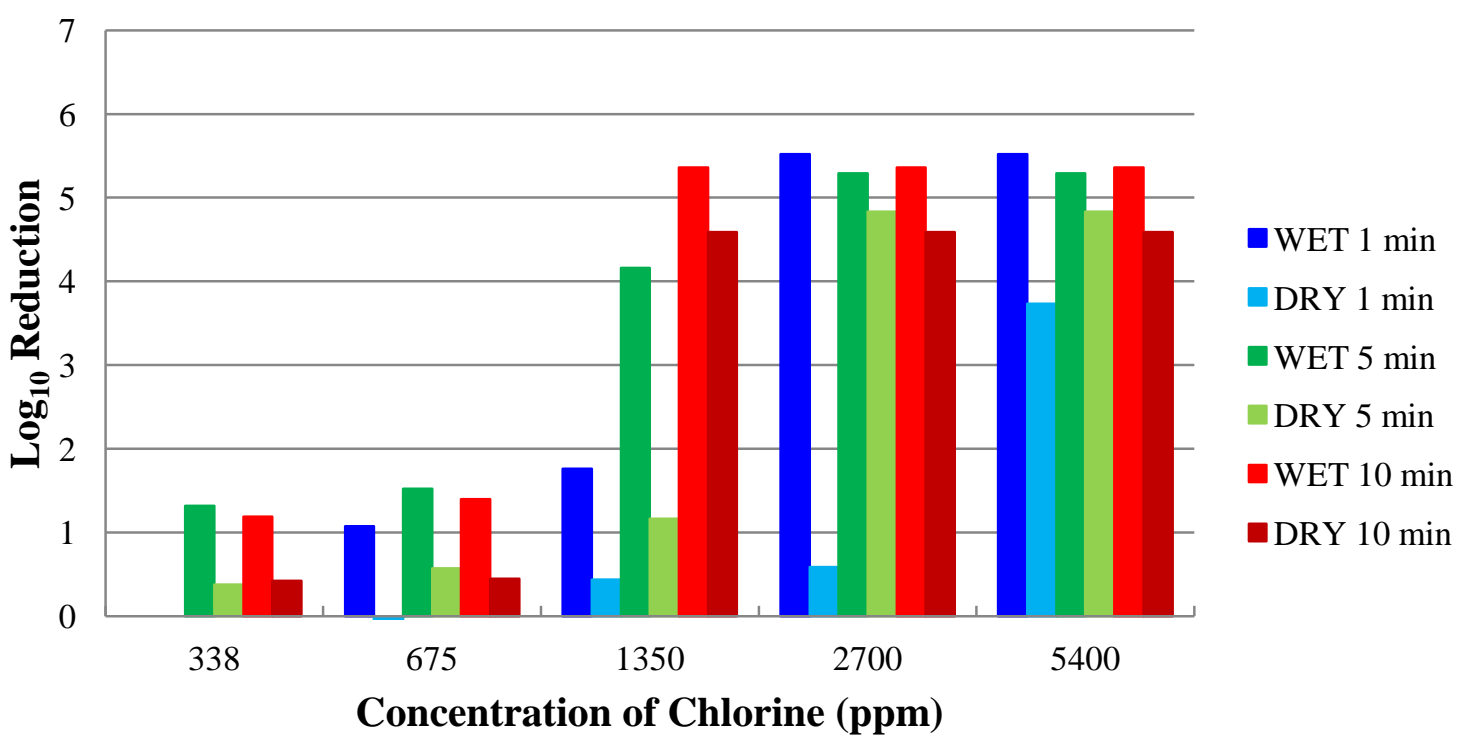

Figure 8. Effectiveness of sodium hypochlorite against FCV, (A) without soil load and (B) with soil load, at 1, 5 and 10 minute contact times, under wet and dry load conditions.

WET refers to wet load conditions and DRY refers to dry load conditions. 
A

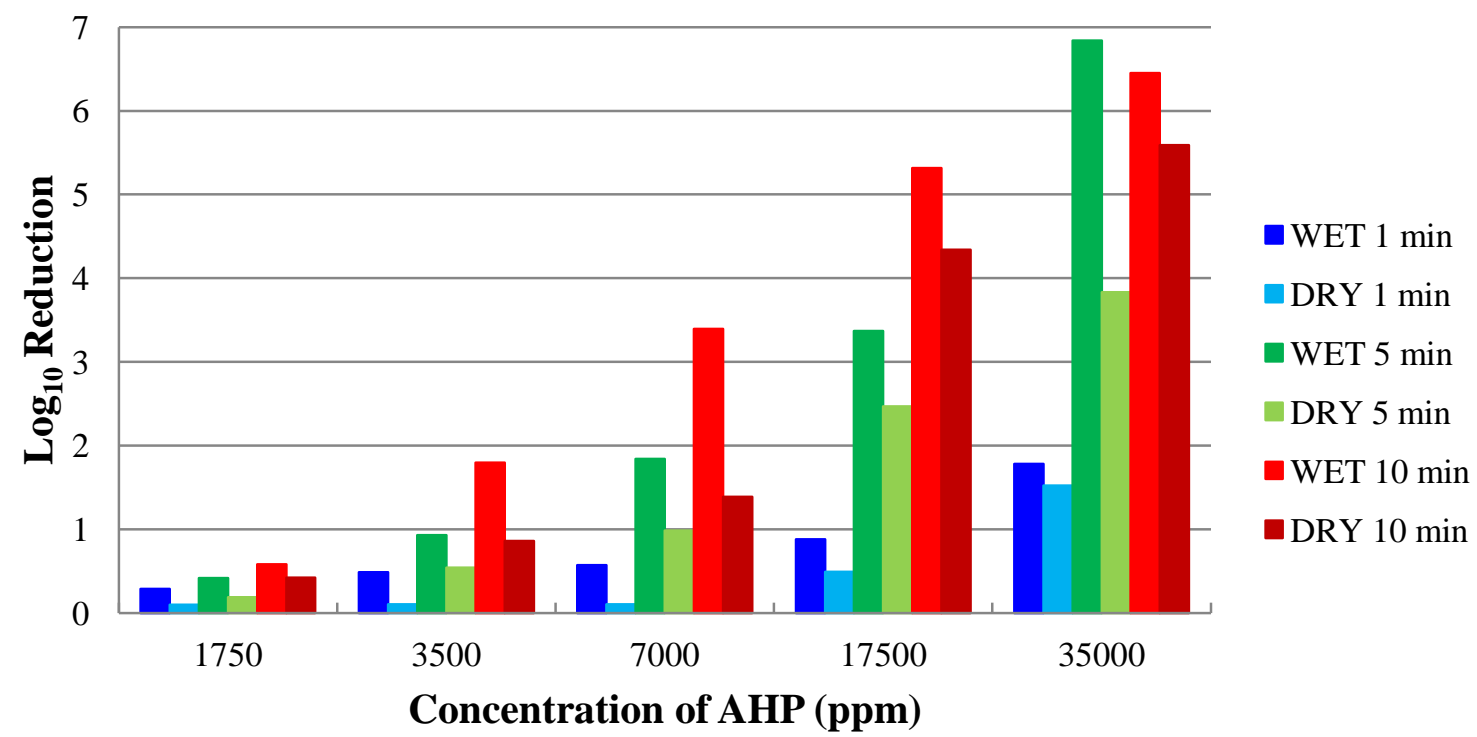

B

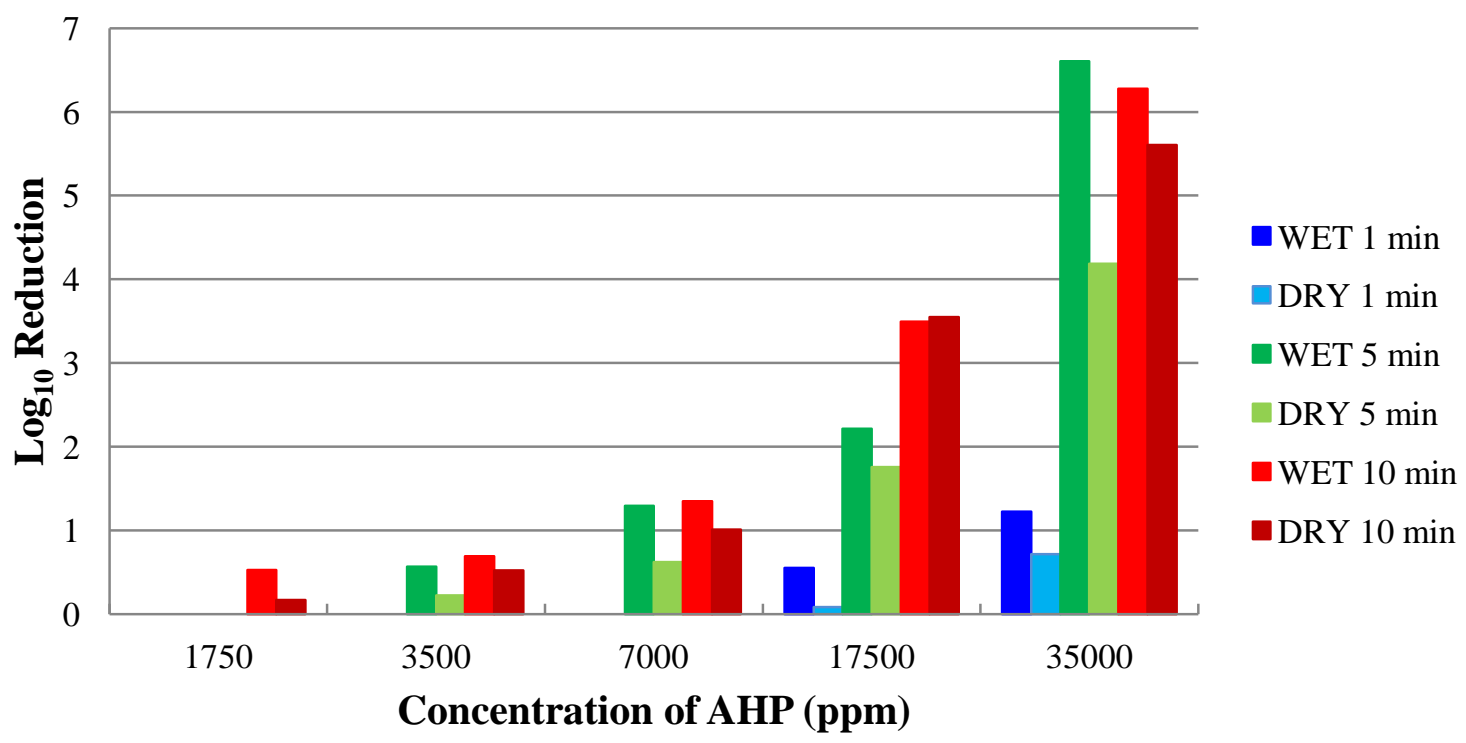

Figure 9. Effectiveness of Accel (AHP) against MNV-1, (A) without soil load and (B) with soil load, at 1, 5 and 10 minute contact times, under wet and dry load conditions. WET refers to wet load conditions and DRY refers to dry load conditions. 
A

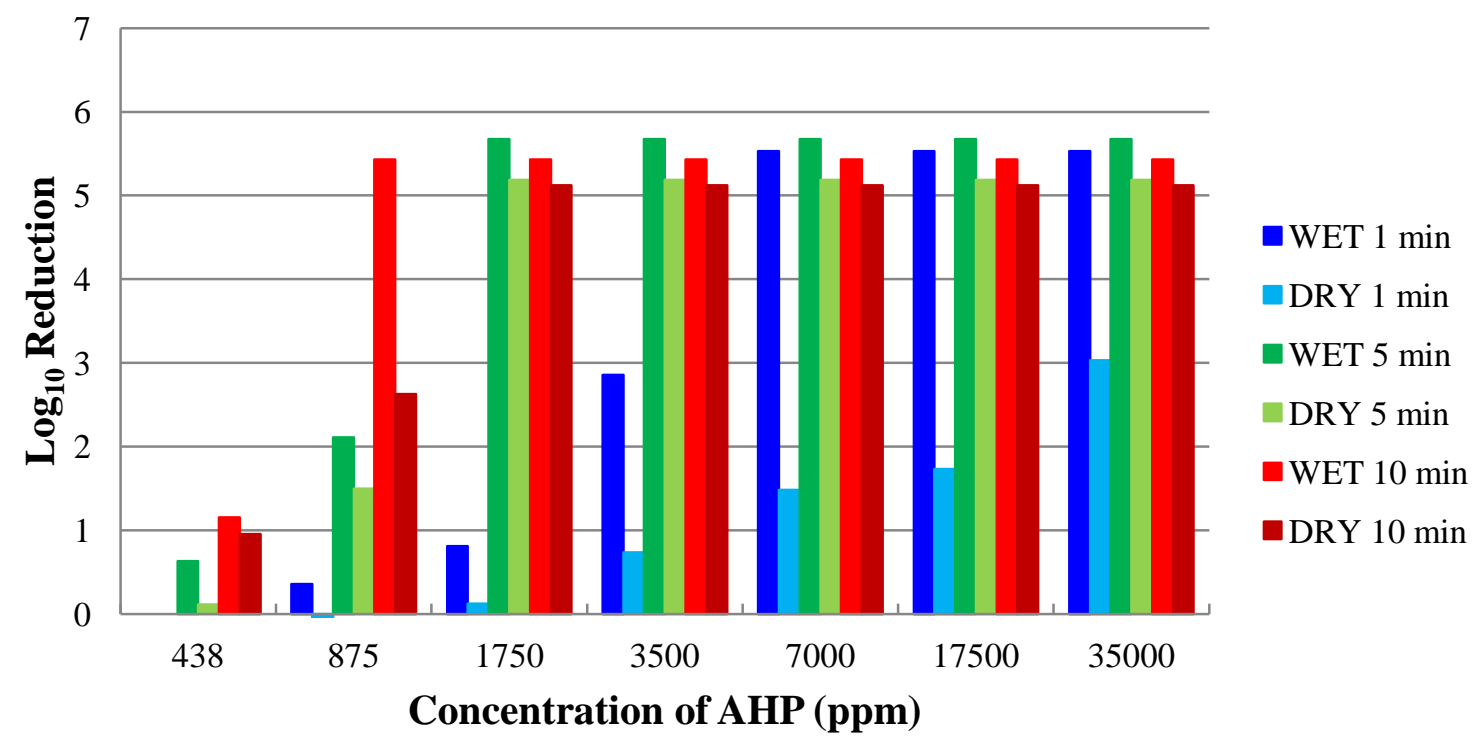

B

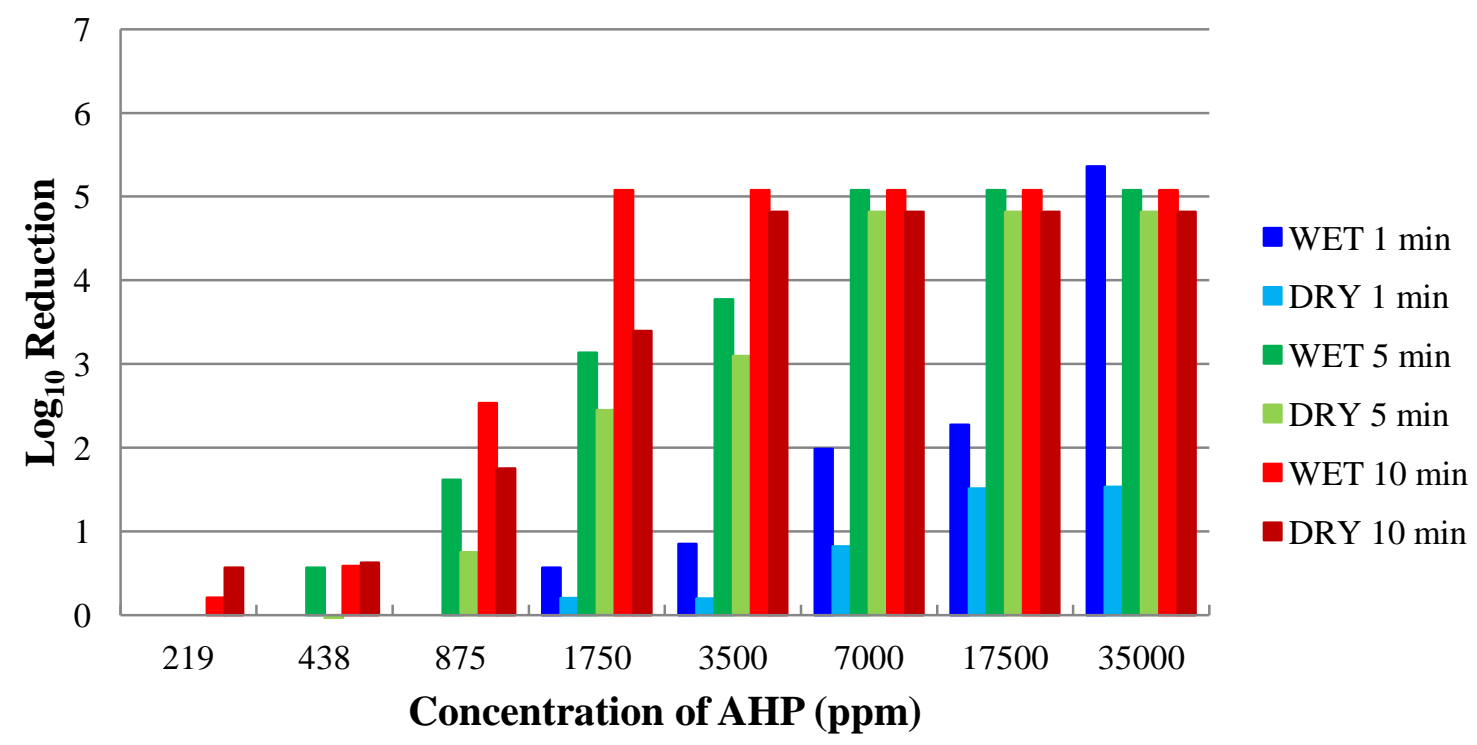

Figure 10. Effectiveness of Accel (AHP) against FCV, (A) without soil load and (B) with soil load, at 1, 5 and 10 minute contact times, under wet and dry load conditions. WET refers to wet load conditions and DRY refers to dry load conditions. 


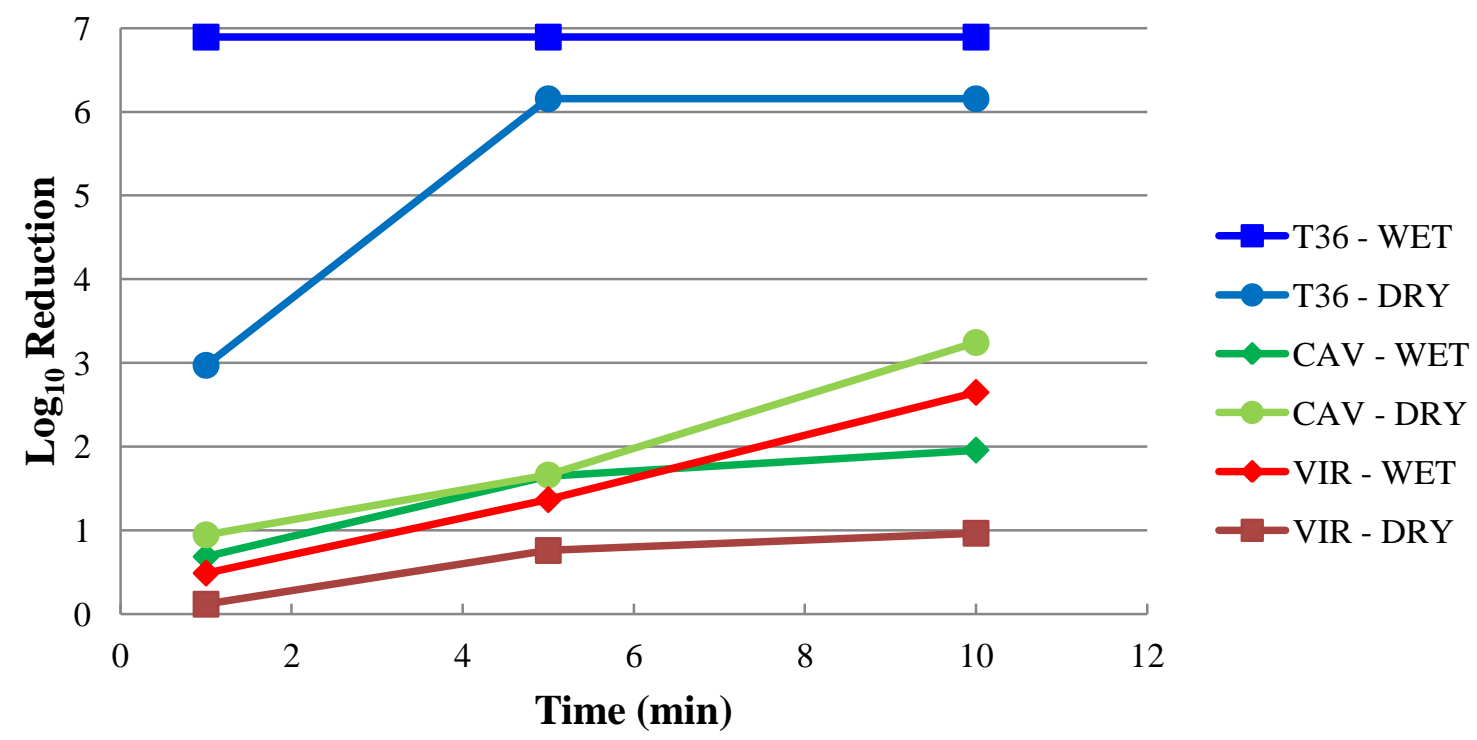

Figure 11. Effectiveness of CaviCide (QUAT), Virox 5 (AHP) and $\mathrm{T}^{3} 6$ (QUAT-alcohol) against MNV-1 (without soil load) at 1, 5 and 10 minute contact times, under wet and dry load conditions.

CAV refers to CaviCide, VIR refers to Virox 5 and T36 refers to $\mathrm{T}^{3} 6$. WET refers to wet load conditions and DRY refers to dry load conditions.

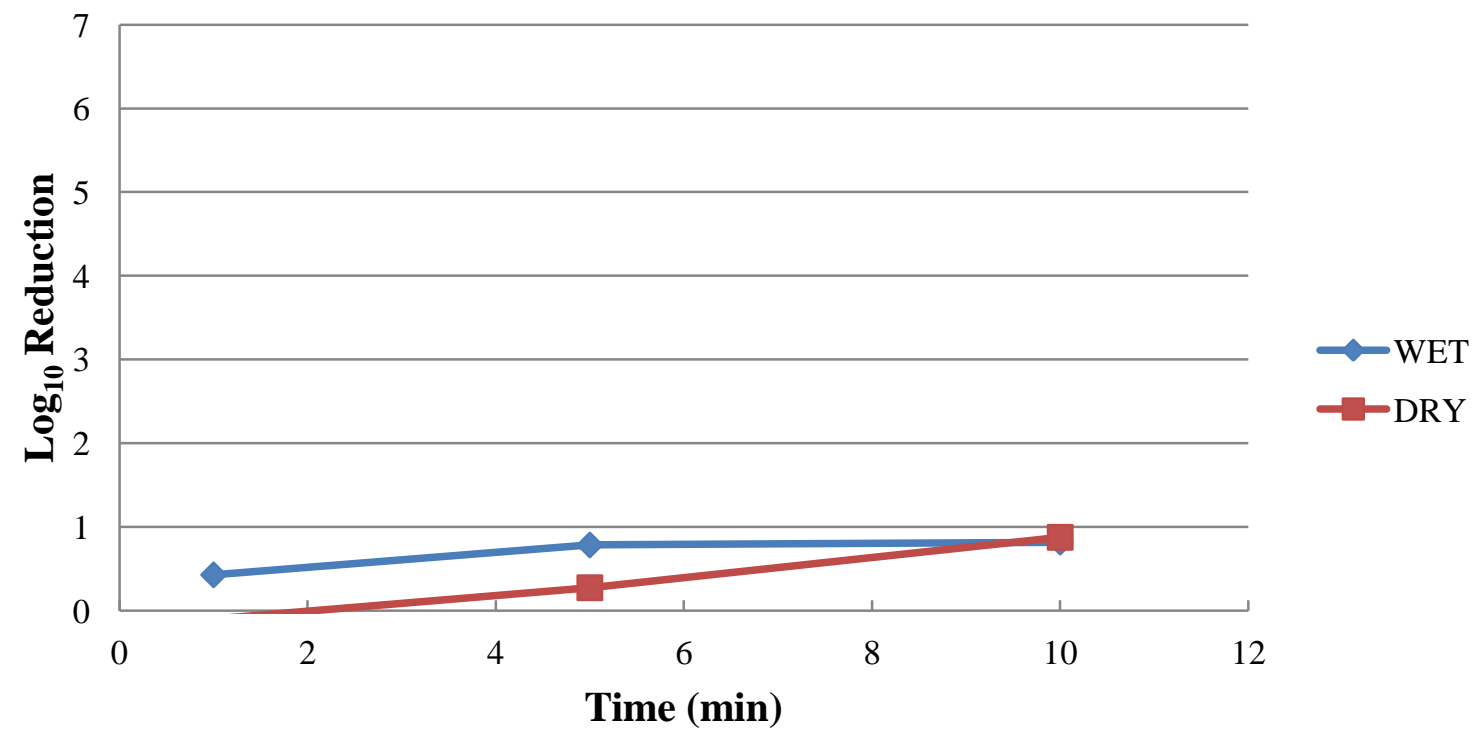

Figure 12. Effectiveness of Virox 5 (AHP) against MNV-1 (with soil load) at 1, 5 and 10 minute contact times, under wet and dry load conditions.

WET refers to wet load conditions and DRY refers to dry load conditions. 


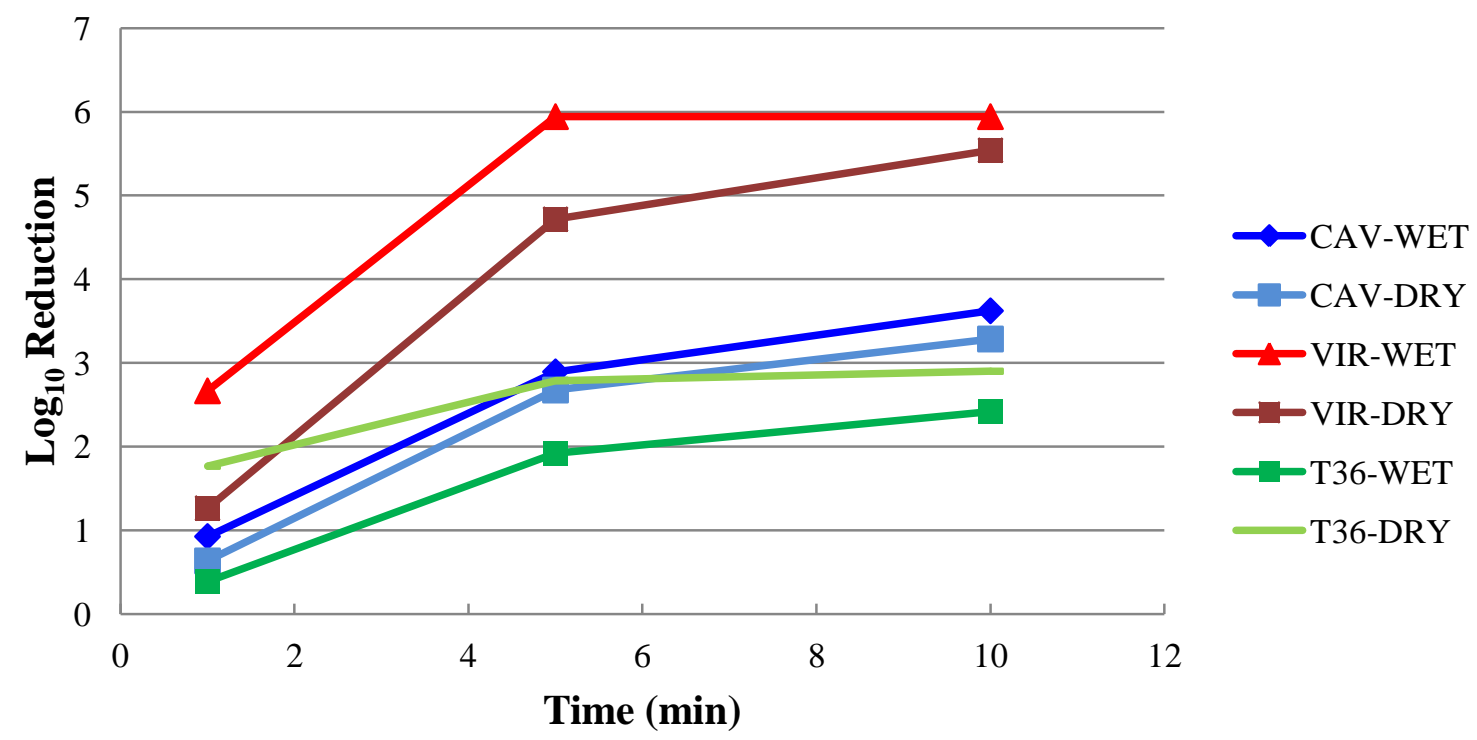

Figure 13. Effectiveness of CaviCide (QUAT), Virox 5 (AHP) and $\mathrm{T}^{3} 6$ (QUAT-alcohol) against FCV (without soil load) at 1, 5 and 10 minute contact times, under wet and dry load conditions. CAV refers to CaviCide, VIR refers to Virox 5 and T36 refers to $T^{3} 6$. WET refers to wet load conditions and DRY refers to dry load conditions.

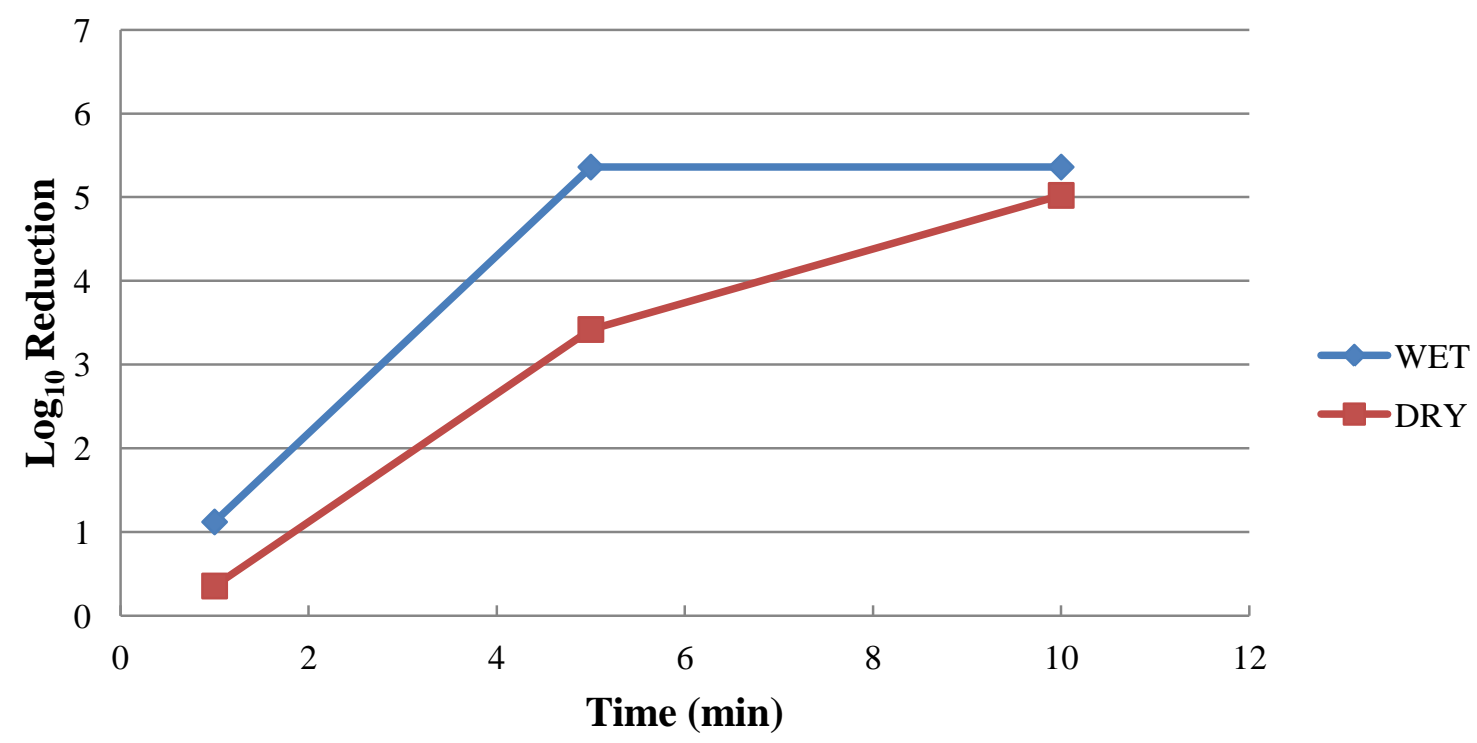

Figure 14. Effectiveness of Virox 5 (AHP) against FCV (with soil load) at 1, 5 and 10 minute contact times, under wet and dry load conditions.

WET refers to wet load conditions and DRY refers to dry load conditions. 
Table 6. Cytotoxicity control test of RAW 264.7 macrophage mouse and CRFK cells from exposure to selected disinfectants and reagents.

\begin{tabular}{|c|c|c|c|c|c|}
\hline \multirow[t]{2}{*}{ Test } & \multicolumn{2}{|c|}{ Test formulation or reagent selected } & \multirow[t]{2}{*}{ Dilution } & \multicolumn{2}{|c|}{ Cytotoxicity observed* } \\
\hline & $50 \mu \mathrm{L}$ & $950 \mu \mathrm{L}$ & & $\begin{array}{c}\text { RAW } 264.7 \\
\text { cells }\end{array}$ & CRFK cells \\
\hline $\begin{array}{l}\text { Disinfectant } \\
\text { (without } \\
\text { neutralizer) }\end{array}$ & $\begin{array}{l}\text { Accel }(1 / 2 \text { dilution })^{\mathrm{a}} \\
\text { Accel }(1 / 4 \text { dilution })^{\mathrm{b}} \\
\text { Bleach }(1 / 10 \text { dilution })^{\mathrm{c}} \\
\text { CaviCide } \\
\text { Virox } 5 \\
\mathrm{~T}^{3} 6 \\
\mathrm{~T}^{3} 6^{\mathrm{d}}\end{array}$ & $\begin{array}{l}\text { EBSS } \\
\text { EBSS } \\
\text { EBSS } \\
\text { EBSS } \\
\text { EBSS } \\
\text { EBSS } \\
\text { EBSS }^{d}\end{array}$ & $\begin{array}{l}10^{-1} \\
10^{-1} \\
10^{-1} \\
10^{-1} \\
10^{-2} \\
10^{-1} \\
10^{-1} \\
10^{-2} \\
10^{-3} \\
10^{-1} \\
10^{-2}\end{array}$ & $\begin{array}{l}<10 \% \\
\text { No } \\
\text { No } \\
100 \% \\
\text { No } \\
\text { No } \\
\text { NT } \\
100 \% \\
\text { No } \\
100 \% \\
\text { No }\end{array}$ & $\begin{array}{l}80-90 \% \\
\text { NT } \\
\text { No } \\
\text { No } \\
\text { NT } \\
\text { No } \\
\text { No } \\
\text { No } \\
\text { NT } \\
\text { No } \\
\text { NT }\end{array}$ \\
\hline $\begin{array}{l}\text { Disinfectant } \\
+ \text { neutralizer }\end{array}$ & $\begin{array}{l}\text { Accel }(1 / 2 \text { dilution })^{\mathrm{a}} \\
\text { Accel }(1 / 4 \text { dilution })^{\mathrm{b}} \\
\text { Bleach }(1 / 10 \text { dilution })^{\mathrm{c}} \\
\text { CaviCide } \\
\text { Virox } 5 \\
\mathrm{~T}^{3} 6 \\
\mathrm{~T}^{3} 6^{\mathrm{d}}\end{array}$ & $\begin{array}{l}L^{f}{ }^{f} \\
L^{f} \\
S T^{e} \\
L^{f} \\
L^{f} \\
L B^{f} \\
\\
L B^{d, f}\end{array}$ & $\begin{array}{l}10^{-1} \\
10^{-1} \\
10^{-1} \\
10^{-1} \\
10^{-2} \\
10^{-1} \\
10^{-1} \\
10^{-2} \\
10^{-3} \\
10^{-1} \\
10^{-2}\end{array}$ & $\begin{array}{l}\text { No } \\
\text { No } \\
\text { No } \\
100 \% \\
\text { No } \\
\text { No } \\
\text { NT } \\
100 \% \\
\text { No } \\
100 \% \\
\text { No }\end{array}$ & $\begin{array}{l}<10 \% \\
\text { No } \\
\text { No } \\
\text { No } \\
\text { NT } \\
\text { No } \\
\text { No } \\
\text { No } \\
\text { NT } \\
\text { No } \\
\text { NT }\end{array}$ \\
\hline Neutralizer & $\begin{array}{l}\mathrm{ST}^{\mathrm{e}} \\
\mathrm{LB}^{\mathrm{f}}\end{array}$ & $\begin{array}{l}\text { EBSS } \\
\text { EBSS }\end{array}$ & $\begin{array}{l}10^{-1} \\
10^{-1}\end{array}$ & $\begin{array}{l}\text { No } \\
\text { No }\end{array}$ & $\begin{array}{l}\text { No } \\
\text { No }\end{array}$ \\
\hline Soil load & Soil load mixture & EBSS & $10^{-1}$ & No & No \\
\hline Control & EBSS & EBSS & $10^{-1}$ & No & No \\
\hline
\end{tabular}

*All trials were performed in triplicate. NT refers to not tested.

${ }^{\mathrm{a}}$ Accel (1/2 dilution) was at $35000 \mathrm{ppm}$.

${ }^{\mathrm{b}}$ Accel (1/4 dilution) was at $17500 \mathrm{ppm}$.

${ }^{\mathrm{c}}$ Bleach refers to Domestic Miraclean sodium hypochlorite at $1 / 10$ dilution or $5400 \mathrm{ppm}$.

${ }^{\mathrm{d}} \mathrm{T}^{3} 6$ was tested at $20 \mu \mathrm{L}$ instead of $50 \mu \mathrm{L}$, and EBSS and LB was tested at $980 \mu \mathrm{L}$ instead of $950 \mu \mathrm{L}$.

${ }^{\mathrm{e}} \mathrm{ST}$ refers to sodium thiosulphate neutralizer.

${ }^{\mathrm{f}} \mathrm{LB}$ refers to letheen broth neutralizer. 
Table 7. Interference with virus infectivity control test of MNV-1 and FCV to infect host cells RAW 264.7 macrophage mouse and CRFK cells from exposure to selected disinfectants and reagents.

\begin{tabular}{|c|c|c|c|c|c|}
\hline \multirow[t]{2}{*}{ Test } & \multicolumn{2}{|c|}{ Test formulation or reagent selected } & \multirow[t]{2}{*}{ Dilution } & \multicolumn{2}{|c|}{ Interference observed* } \\
\hline & $50 \mu \mathrm{L}$ & $950 \mu \mathrm{L}$ & & MNV-1 & $\mathrm{FCV}$ \\
\hline $\begin{array}{l}\text { Disinfectant } \\
\text { (without } \\
\text { neutralizer) }\end{array}$ & $\begin{array}{l}\text { Accel }(1 / 2 \text { dilution })^{\mathrm{a}} \\
\text { Accel }(1 / 4 \text { dilution })^{\mathrm{b}} \\
\text { Bleach }(1 / 10 \text { dilution })^{\mathrm{c}} \\
\text { CaviCide } \\
\text { Virox } 5 \\
\mathrm{~T}^{3} 6 \\
\mathrm{~T}^{3} 6^{\mathrm{d}}\end{array}$ & $\begin{array}{l}\text { EBSS } \\
\text { EBSS } \\
\text { EBSS } \\
\text { EBSS } \\
\text { EBSS } \\
\text { EBSS } \\
\text { EBSS }\end{array}$ & $\begin{array}{l}10^{-1} \\
10^{-1} \\
10^{-1} \\
10^{-1} \\
10^{-2} \\
10^{-1} \\
10^{-1} \\
10^{-1} \\
10^{-2}\end{array}$ & $\begin{array}{l}\text { CTE } \\
\text { Yes }^{g} \\
\text { No } \\
\text { NT } \\
\text { No } \\
\text { No } \\
\text { NT } \\
\text { NT } \\
\text { No }\end{array}$ & $\begin{array}{l}\text { CTE } \\
\text { NT } \\
\text { Yes }^{g} \\
\text { No } \\
\text { NT } \\
\text { No } \\
\text { No } \\
\text { No } \\
\text { NT }\end{array}$ \\
\hline $\begin{array}{l}\text { Disinfectant } \\
+ \text { neutralizer }\end{array}$ & $\begin{array}{l}\text { Accel }(1 / 2 \text { dilution })^{\mathrm{a}} \\
\text { Accel }(1 / 4 \text { dilution })^{\mathrm{b}} \\
\text { Bleach }(1 / 10 \text { dilution })^{\mathrm{c}} \\
\text { CaviCide } \\
\text { Virox } 5 \\
\mathrm{~T}^{3} 6 \\
\mathrm{~T}^{3} 6^{\mathrm{d}}\end{array}$ & $\begin{array}{l}\mathrm{LB}^{\mathrm{f}} \\
\mathrm{LB}^{\mathrm{f}} \\
\mathrm{ST}^{\mathrm{e}} \\
\mathrm{LB}^{\mathrm{f}} \\
\mathrm{LB}^{\mathrm{f}} \\
\mathrm{LB}^{\mathrm{f}} \\
\mathrm{LB}^{\mathrm{d}, \mathrm{f}}\end{array}$ & $\begin{array}{l}10^{-1} \\
10^{-1} \\
10^{-1} \\
10^{-1} \\
10^{-2} \\
10^{-1} \\
10^{-1} \\
10^{-1} \\
10^{-2}\end{array}$ & $\begin{array}{l}\text { Yes }^{g} \\
\text { Yes }^{g} \\
\text { No } \\
\text { NT } \\
\text { No } \\
\text { No } \\
\text { NT } \\
\text { NT } \\
\text { No }\end{array}$ & $\begin{array}{l}\text { No } \\
\text { NT } \\
\text { No } \\
\text { No } \\
\text { NT } \\
\text { Yes }^{g} \\
\text { Yes }^{g} \\
\text { No } \\
\text { NT }\end{array}$ \\
\hline Neutralizer & $\begin{array}{l}\mathrm{ST}^{\mathrm{e}} \\
\mathrm{LB}^{\mathrm{f}}\end{array}$ & $\begin{array}{l}\text { EBSS } \\
\text { EBSS }\end{array}$ & $\begin{array}{l}10^{-1} \\
10^{-1}\end{array}$ & $\begin{array}{l}\text { No } \\
\text { No }\end{array}$ & $\begin{array}{l}\text { No } \\
\text { No }\end{array}$ \\
\hline Soil load & Soil load mixture & EBSS & $10^{-1}$ & No & No \\
\hline Control & EBSS & EBSS & $10^{-1}$ & NA & NA \\
\hline
\end{tabular}

*All trials were performed in triplicate. NT refers to not tested. NA refers to not applicable. Host cell for MNV-1 was RAW 264.7 macrophage mouse cells and host cell for FCV was CRFK cells. CTE refers to cytotoxic effects.

${ }^{\mathrm{a}}$ Accel (1/2 dilution) was at $35000 \mathrm{ppm}$.

${ }^{\mathrm{b}}$ Accel (1/4 dilution) was at $17500 \mathrm{ppm}$.

${ }^{\mathrm{c}}$ Bleach refers to Domestic Miraclean sodium hypochlorite at $1 / 10$ dilution or $5400 \mathrm{ppm}$.

${ }^{\mathrm{d}} \mathrm{T}^{3} 6$ was tested at $20 \mu \mathrm{L}$ instead of $50 \mu \mathrm{L}$, and EBSS and LB was tested at $980 \mu \mathrm{L}$ instead of $950 \mu \mathrm{L}$.

${ }^{\mathrm{e}} \mathrm{ST}$ refers to sodium thiosulphate neutralizer.

${ }^{\mathrm{f}} \mathrm{LB}$ refers to letheen broth neutralizer.

${ }^{\mathrm{g}}$ Interference observed that was positive was statistically significant $(\mathrm{p}<0.05)$. 
Table 8. Mean log and percentage differences in viral titer after drying for MNV-1 and FCV controls.

\begin{tabular}{|c|c|c|c|c|c|c|c|c|c|c|c|}
\hline \multirow{2}{*}{$\begin{array}{c}\text { Virus } \\
\text { MNV-1 }\end{array}$} & \multirow{2}{*}{$\begin{array}{l}\text { Soil } \\
\text { load } \\
\text { No }\end{array}$} & \multirow{5}{*}{$\begin{array}{l}\text { Disinfectant } \\
\text { Domestic } \\
\text { Miraclean } \\
\text { Bleach }\end{array}$} & \multicolumn{2}{|c|}{$\begin{array}{c}\text { Wet load } \\
\text { (PFU/mL, log) }\end{array}$} & \multicolumn{2}{|c|}{$\begin{array}{c}\text { Dry load } \\
\text { (PFU/mL, log) }\end{array}$} & \multirow{5}{*}{$\begin{array}{c}\begin{array}{c}\text { Viral titer } \\
\text { remaining* }(\%)\end{array} \\
14.0 \\
15.2 \\
31.5 \\
13.9\end{array}$} & \multirow{5}{*}{$\begin{array}{c}\begin{array}{c}\text { Viral titer } \\
\text { decrease }^{* *}(\%)\end{array} \\
86.0 \\
84.8 \\
68.5 \\
86.1\end{array}$} & \multirow{2}{*}{$\begin{array}{c}\text { Mean viral titer } \\
\text { remaining }(\%)\end{array}$} & \multirow{2}{*}{$\begin{array}{c}\begin{array}{c}\text { Wet and dry load } \\
\text { difference } * * *(\log )\end{array} \\
0.85\end{array}$} & \multirow{2}{*}{$\begin{array}{c}\text { Mean difference } \\
(\log )\end{array}$} \\
\hline & & & 5.7E6 & 6.8 & $8.0 \mathrm{E} 5$ & 5.9 & & & & & \\
\hline & & & 4.4E6 & 6.6 & $6.7 \mathrm{E} 5$ & 5.8 & & & & 0.82 & \\
\hline & & & $1.1 \mathrm{E} 6$ & 6.0 & $3.4 \mathrm{E} 5$ & 5.5 & & & & 0.50 & \\
\hline & & & 2.7E6 & 6.4 & 3.7E5 & 5.6 & & & & 0.86 & \\
\hline & & Accel & $3.6 \mathrm{E} 6$ & 6.6 & $9.5 \mathrm{E} 5$ & 6.0 & 26.2 & 73.8 & & 0.58 & \\
\hline & & & $6.9 \mathrm{E} 6$ & 6.8 & $1.2 \mathrm{E} 6$ & 6.1 & 16.9 & 83.1 & & 0.77 & \\
\hline & & & $3.2 \mathrm{E} 6$ & 6.5 & 7.9E5 & 5.9 & 24.9 & 75.1 & & 0.60 & \\
\hline & & & $2.8 \mathrm{E} 6$ & 6.5 & $3.9 \mathrm{E} 5$ & 5.6 & 13.7 & 86.3 & & 0.86 & \\
\hline & & Virox 5 & 2.7E6 & 6.4 & 7.7E5 & 5.9 & 28.5 & 71.5 & & 0.55 & \\
\hline & & CaviCide & $4.0 \mathrm{E} 6$ & 6.6 & $7.0 \mathrm{E} 5$ & 5.9 & 17.6 & 82.4 & & 0.75 & \\
\hline & & $\mathrm{T}^{3} 6$ & 7.8E6 & 6.9 & $1.4 \mathrm{E} 6$ & 6.2 & 18.3 & 81.7 & & 0.74 & \\
\hline & Yes & Domestic & $2.8 \mathrm{E} 6$ & 6.5 & $2.9 \mathrm{E} 5$ & 5.5 & 10.4 & 89.7 & 17.6 & 0.98 & 0.77 \\
\hline & & $\begin{array}{l}\text { Miraclean } \\
\text { Bleach }\end{array}$ & $1.8 \mathrm{E} 6$ & 6.3 & $2.9 \mathrm{E} 5$ & 5.5 & 15.8 & 84.2 & & 0.80 & \\
\hline & & Accel & $4.0 \mathrm{E} 6$ & 6.6 & $1.0 \mathrm{E} 6$ & 6.0 & 25.3 & 74.8 & & 0.60 & \\
\hline & & CaviCide & NT & NT & NT & NT & - & - & & - & \\
\hline & & $\mathrm{T}^{3} 6$ & NT & NT & NT & NT & - & - & & - & \\
\hline \multirow[t]{18}{*}{ FCV } & \multirow[t]{11}{*}{ No } & Domestic & $4.4 \mathrm{E} 5$ & 5.7 & $2.4 \mathrm{E} 5$ & 5.4 & 52.9 & 47.1 & \multirow[t]{11}{*}{43.2} & 0.28 & \multirow[t]{11}{*}{0.39} \\
\hline & & Miraclean & 4.4E5 & 5.7 & $1.6 \mathrm{E} 5$ & 5.2 & 35.1 & 64.9 & & 0.45 & \\
\hline & & Bleach & $1.5 \mathrm{E} 5$ & 5.2 & $6.1 \mathrm{E} 4$ & 4.8 & 41.0 & 59.0 & & 0.39 & \\
\hline & & & $4.0 \mathrm{E} 5$ & 5.6 & $7.1 \mathrm{E} 4$ & 4.9 & 17.7 & 82.4 & & 0.75 & \\
\hline & & & $3.6 \mathrm{E} 5$ & 5.6 & $2.2 \mathrm{E} 5$ & 5.3 & 59.3 & 40.7 & & 0.23 & \\
\hline & & Accel & $3.4 \mathrm{E} 5$ & 5.5 & $1.1 \mathrm{E} 5$ & 5.0 & 31.8 & 68.3 & & 0.50 & \\
\hline & & & 4.7E5 & 5.7 & $1.5 \mathrm{E} 5$ & 5.2 & 32.5 & 67.5 & & 0.49 & \\
\hline & & & 2.7E5 & 5.4 & $1.3 \mathrm{E} 5$ & 5.1 & 49.4 & 50.6 & & 0.31 & \\
\hline & & Virox 5 & $8.8 \mathrm{E} 5$ & 6.0 & $3.5 \mathrm{E} 5$ & 5.5 & 39.7 & 60.3 & & 0.40 & \\
\hline & & CaviCide & $8.8 \mathrm{E} 5$ & 6.0 & $3.5 \mathrm{E} 5$ & 5.5 & 39.7 & 60.3 & & 0.40 & \\
\hline & & $T^{3} 6$ & $4.0 \mathrm{E} 5$ & 5.6 & $3.1 \mathrm{E} 5$ & 5.5 & 76.0 & 24.0 & & 0.12 & \\
\hline & \multirow[t]{7}{*}{ Yes } & Domestic & $3.3 \mathrm{E} 5$ & 5.5 & $1.1 \mathrm{E} 5$ & 5.0 & 32.7 & 67.3 & \multirow[t]{7}{*}{37.9} & 0.49 & \multirow[t]{7}{*}{0.45} \\
\hline & & Miraclean & $8.8 \mathrm{E} 4$ & 4.9 & $2.9 \mathrm{E} 4$ & 4.5 & 33.5 & 66.5 & & 0.48 & \\
\hline & & Bleach & $2.0 \mathrm{E} 5$ & 5.3 & $6.8 \mathrm{E} 4$ & 4.8 & 34.9 & 65.1 & & 0.46 & \\
\hline & & & $1.2 \mathrm{E} 5$ & 5.1 & $6.6 \mathrm{E} 4$ & 4.8 & 55.5 & 44.5 & & 0.26 & \\
\hline & & Virox 5 & $2.3 \mathrm{E} 5$ & 5.4 & $1.1 \mathrm{E} 5$ & 5.0 & 45.9 & 54.2 & & 0.34 & \\
\hline & & CaviCide & NT & NT & NT & NT & - & - & & - & \\
\hline & & $\mathrm{T}^{3} 6$ & NT & NT & NT & NT & - & - & & - & \\
\hline
\end{tabular}

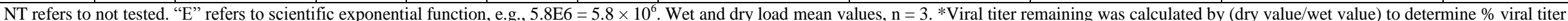

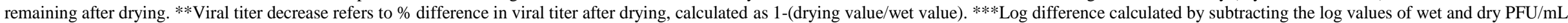
values. 
Table 9. Summary of the most effective concentrations and contact times of commonly used disinfectants against MNV-1 and FCV, surrogates of human norovirus, with and without soil load.

\begin{tabular}{|c|c|c|c|c|}
\hline \multirow[t]{3}{*}{ Disinfectant } & \multicolumn{4}{|c|}{ Wet and dry load range $\left(\log _{10} \text { reduction }\right)^{\mathrm{a}}$, concentration $(\mathrm{ppm})$ and contact time (min) } \\
\hline & \multicolumn{2}{|c|}{ MNV-1 } & \multicolumn{2}{|c|}{ FCV } \\
\hline & Without soil load & With soil load & Without soil load & With soil load \\
\hline \multirow[t]{3}{*}{$\begin{array}{l}\text { Domestic Miraclean } \\
\text { Bleach }\end{array}$} & $\begin{array}{l}\text { 6.76, 5.90 } \\
2700 \mathrm{ppm}, 1 \mathrm{~min}\end{array}$ & $\begin{array}{l}\text { 6.44, } \mathbf{6 . 6 8} \\
5400 \mathrm{ppm}, 1 \mathrm{~min}\end{array}$ & $\begin{array}{l}\mathbf{5 . 6 5}, \mathbf{5 . 3 7} \\
5400 \mathrm{ppm}, 1 \mathrm{~min}\end{array}$ & \multirow[t]{2}{*}{$\begin{array}{l}\mathbf{5 . 2 9}, \mathbf{4 . 8 3} \\
2700 \mathrm{ppm}, 5 \mathrm{~min}\end{array}$} \\
\hline & $\begin{array}{l}\mathbf{6 . 0 3}, \mathbf{5 . 5 3} \\
1350 \mathrm{ppm}, 5 \mathrm{~min}\end{array}$ & \multirow[t]{2}{*}{$\begin{array}{l}\mathbf{6 . 4 5}, \mathbf{5 . 4 7} \\
1350 \mathrm{ppm}, 5 \mathrm{~min}\end{array}$} & $\begin{array}{l}\mathbf{4 . 6 1}, \mathbf{4 . 8 5} \\
1350 \mathrm{ppm}, 5 \mathrm{~min}\end{array}$ & \\
\hline & $\begin{array}{l}\mathbf{6 . 4 3}, \mathbf{5 . 5 7} \\
675 \mathrm{ppm}, 10 \mathrm{~min}\end{array}$ & & $\begin{array}{l}\mathbf{5 . 5 6}, \mathbf{5 . 3 3} \\
1350 \mathrm{ppm}, 10 \mathrm{~min}\end{array}$ & $\begin{array}{l}\mathbf{5 . 3 6}, \mathbf{4 . 5 9} \\
1350 \mathrm{ppm}, 10 \mathrm{~min}\end{array}$ \\
\hline \multirow[t]{2}{*}{ Accel } & \multirow[t]{2}{*}{$\begin{array}{l}\mathbf{6 . 4 5}, \mathbf{5 . 5 9} \text { (est) } \\
35000 \mathrm{ppm}, 10 \mathrm{~min}\end{array}$} & \multirow[t]{2}{*}{$\begin{array}{l}\mathbf{6 . 2 8}, \mathbf{5 . 6 0} \text { (est) } \\
35000 \mathrm{ppm}, 10 \mathrm{~min}\end{array}$} & \multirow[t]{2}{*}{$\begin{array}{l}\mathbf{5 . 6 7 , 5 . 1 8} \\
1750 \mathrm{ppm}, 5 \mathrm{~min}\end{array}$} & $\begin{array}{l}\mathbf{5 . 0 8 ,} \mathbf{4 . 8 2} \\
7000 \mathrm{ppm}, 5 \mathrm{~min}\end{array}$ \\
\hline & & & & $\begin{array}{l}\mathbf{5 . 0 8 , 4 . 8 2} \\
3500 \mathrm{ppm}, 10 \mathrm{~min}\end{array}$ \\
\hline Virox 5 & $\begin{array}{l}2.64,0.96 \\
5000 \mathrm{ppm}, 10 \mathrm{~min}\end{array}$ & $\begin{array}{l}0.82,0.87 \\
5000 \mathrm{ppm}, 10 \mathrm{~min}\end{array}$ & $\begin{array}{l}\mathbf{5 . 9 4 , 5 . 5 4} \\
5000 \mathrm{ppm}, 10 \mathrm{~min}\end{array}$ & $\begin{array}{l}\mathbf{5 . 3 6 , 5 . 0 2} \\
5000 \mathrm{ppm}, 10 \mathrm{~min}\end{array}$ \\
\hline CaviCide & $\begin{array}{l}1.96,3.24 \\
2800 \mathrm{ppm}, 10 \mathrm{~min}\end{array}$ & NT & $\begin{array}{l}3.62,3.29 \\
2800 \mathrm{ppm}, 10 \mathrm{~min}\end{array}$ & NT \\
\hline $\mathrm{T}^{3} 6$ & $\begin{array}{l}\text { 6.89, 6.16 (est) } \\
2000 \text { ppm, } 70 \% \text { ethanol, } 5 \text { min }\end{array}$ & NT & $\begin{array}{l}2.42,2.90 \\
2000 \mathrm{ppm}, 70 \% \text { ethanol, } 10 \mathrm{~min}\end{array}$ & NT \\
\hline
\end{tabular}

Bold mean values refer to inactivation with no virus plaques observed. Experimental trials performed in triplicate.

${ }^{a} \log _{10}$ reduction refers to "wet load, dry load" conditions.

NT refers to not tested. Est refers to estimated value. 
Table 10. Virucidal efficacy of commonly used disinfectants against MNV-1, a surrogate of human norovirus, under wet and dry load conditions, with and without soil load, at 1, 5 and 10 minute contact times.

\begin{tabular}{|c|c|c|c|c|c|c|c|c|c|c|c|c|c|c|}
\hline \multirow[t]{5}{*}{ Disinfectant } & \multirow[t]{5}{*}{ Dilution } & \multirow{5}{*}{$\begin{array}{l}\text { Concentration } \\
(\mathrm{ppm})\end{array}$} & \multicolumn{12}{|c|}{$\log _{10}$ reduction } \\
\hline & & & \multicolumn{6}{|c|}{ Wet load conditions } & \multicolumn{6}{|c|}{ Dry load conditions } \\
\hline & & & \multicolumn{3}{|c|}{ Without soil load } & \multicolumn{3}{|c|}{ With soil load } & \multicolumn{3}{|c|}{ Without soil load } & \multicolumn{3}{|c|}{ With soil load } \\
\hline & & & \multicolumn{6}{|c|}{ Contact time $(\mathrm{min})$} & \multicolumn{6}{|c|}{ Contact time $(\mathrm{min})$} \\
\hline & & & 1 & 5 & 10 & 1 & 5 & 10 & 1 & 5 & 10 & 1 & 5 & 10 \\
\hline \multirow{8}{*}{$\begin{array}{l}\text { Domestic } \\
\text { Miraclean } \\
\text { Bleach }\end{array}$} & $1 / 10$ & 5400 & 6.76 & 6.03 & 6.43 & 6.44 & 6.45 & 6.26 & 5.90 & 5.53 & 5.57 & 6.68 & 5.47 & 5.46 \\
\hline & $1 / 20$ & 2700 & 6.76 & 6.03 & 6.43 & 6.44 & 6.45 & 6.26 & 5.90 & 5.53 & 5.57 & 5.86 & 5.47 & 5.46 \\
\hline & $1 / 40$ & 1350 & 4.75 & 6.03 & 6.43 & 4.83 & 6.45 & 6.26 & 2.14 & 5.53 & 5.57 & 3.43 & 5.47 & 5.46 \\
\hline & $1 / 80$ & 675 & 1.91 & 4.13 & 6.43 & 4.03 & 5.62 & 6.26 & 1.40 & 4.41 & 5.57 & 1.80 & 4.34 & 4.63 \\
\hline & $1 / 160$ & 338 & 1.02 & 2.03 & 4.17 & 1.53 & 2.72 & 4.05 & 0.47 & 2.50 & 3.47 & 1.49 & 2.69 & 4.33 \\
\hline & $1 / 320$ & 169 & 0.60 & 0.90 & 1.70 & 0.54 & 1.37 & 2.23 & 0.36 & 0.57 & 1.97 & 1.18 & 0.75 & 2.27 \\
\hline & $1 / 640$ & 84 & 0.58 & 0.57 & 0.53 & NT & 0.41 & 0.61 & 0.11 & -0.12 & 0.17 & NT & 0.03 & 0.96 \\
\hline & $1 / 1280$ & 42 & NT & NT & 0.30 & NT & NT & NT & NT & NT & 0.12 & NT & NT & NT \\
\hline \multirow[t]{5}{*}{ Accel } & $1 / 2$ & 35000 & 1.78 & $6.84 *$ & $6.45^{*}$ & 1.22 & $6.61 *$ & $6.28 *$ & 1.52 & 3.83 & 5.59 & 0.72 & 4.18 & 5.60 \\
\hline & $1 / 4$ & 17500 & 0.88 & 3.37 & 5.31 & 0.55 & 2.21 & 3.49 & 0.49 & 2.47 & 4.34 & 0.08 & 1.75 & 3.55 \\
\hline & $1 / 10$ & 7000 & 0.57 & 1.84 & 3.39 & NT & 1.29 & 1.35 & 0.10 & 0.98 & 1.39 & NT & 0.62 & 1.01 \\
\hline & $1 / 20$ & 3500 & 0.49 & 0.93 & 1.80 & NT & 0.56 & 0.69 & 0.10 & 0.54 & 0.86 & NT & 0.22 & 0.52 \\
\hline & $1 / 40$ & 1750 & 0.28 & 0.42 & 0.58 & NT & NT & 0.52 & 0.10 & 0.19 & 0.42 & NT & NT & 0.17 \\
\hline Virox 5 & Undiluted & 5000 & 0.49 & 1.37 & 2.65 & 0.43 & 0.78 & 0.82 & 0.12 & 0.76 & 0.96 & -0.10 & 0.27 & 0.87 \\
\hline CaviCide & Undiluted & 2800 & 0.68 & 1.65 & 1.96 & NT & NT & NT & 0.95 & 1.66 & 3.24 & NT & NT & NT \\
\hline$T^{3} 6$ & Undiluted & 2000 & 6.89* & $6.89 *$ & $6.89 *$ & NT & NT & NT & 2.97 & 6.16 & 6.16 & NT & NT & NT \\
\hline
\end{tabular}

Bold mean values refer to inactivation of the surrogate virus. Experimental trials were performed in triplicate.

NT refers to not tested. *Refers to estimated value. 
Table 11. Virucidal efficacy of commonly used disinfectants against FCV, a surrogate of human norovirus, under wet and dry load conditions, with and without soil load, at 1, 5 and 10 minute contact times.

\begin{tabular}{|c|c|c|c|c|c|c|c|c|c|c|c|c|c|c|}
\hline \multirow[t]{5}{*}{ Disinfectant } & \multirow[t]{5}{*}{ Dilution } & \multirow{5}{*}{$\begin{array}{c}\text { Concentration } \\
\text { (ppm) }\end{array}$} & \multicolumn{12}{|c|}{$\log _{10}$ reduction } \\
\hline & & & \multicolumn{6}{|c|}{ Wet load conditions } & \multicolumn{6}{|c|}{ Dry load conditions } \\
\hline & & & \multicolumn{3}{|c|}{ Without soil load } & \multicolumn{3}{|c|}{ With soil load } & \multicolumn{3}{|c|}{ Without soil load } & \multicolumn{3}{|c|}{ With soil load } \\
\hline & & & \multicolumn{6}{|c|}{ Contact time (min) } & \multicolumn{6}{|c|}{ Contact time (min) } \\
\hline & & & 1 & 5 & 10 & 1 & 5 & 10 & 1 & 5 & 10 & 1 & 5 & 10 \\
\hline \multirow{6}{*}{$\begin{array}{l}\text { Domestic } \\
\text { Miraclean } \\
\text { Bleach }\end{array}$} & $1 / 10$ & 5400 & 5.65 & 4.61 & 5.56 & 5.52 & 5.29 & 5.36 & 5.37 & 4.85 & 5.33 & 3.73 & 4.83 & 4.59 \\
\hline & $1 / 20$ & 2700 & 5.65 & \begin{tabular}{|l|}
4.61 \\
\end{tabular} & 5.56 & 5.52 & 5.29 & 5.36 & 3.89 & 4.85 & 5.33 & 0.59 & 4.83 & 4.59 \\
\hline & $1 / 40$ & 1350 & 1.86 & 4.61 & 5.56 & 1.76 & 4.16 & 5.36 & 0.94 & 4.85 & 5.33 & 0.44 & 1.16 & 4.59 \\
\hline & $1 / 80$ & 675 & 1.09 & \begin{tabular}{|l|}
0.72 \\
\end{tabular} & 1.87 & 1.07 & 1.52 & 1.40 & 0.17 & 0.53 & 0.78 & -0.20 & 0.57 & 0.45 \\
\hline & $1 / 160$ & 338 & NT & 0.27 & 1.29 & NT & 1.32 & 1.19 & NT & 0.25 & 0.74 & NT & 0.37 & 0.42 \\
\hline & $1 / 320$ & 169 & NT & 0.05 & 0.93 & NT & NT & NT & NT & 0.28 & 0.47 & NT & NT & NT \\
\hline \multirow[t]{8}{*}{ Accel } & $1 / 2$ & 35000 & 5.53 & 5.67 & 5.43 & 5.36 & 5.08 & 5.08 & 3.03 & 5.18 & 5.12 & 1.53 & 4.82 & 4.82 \\
\hline & $1 / 4$ & 17500 & 5.53 & \begin{tabular}{|l|}
5.67 \\
\end{tabular} & 5.43 & 2.27 & 5.08 & 5.08 & 1.73 & 5.18 & 5.12 & 1.51 & 4.82 & 4.82 \\
\hline & $1 / 10$ & 7000 & 5.53 & \begin{tabular}{|l|}
5.67 \\
\end{tabular} & 5.43 & 1.99 & 5.08 & 5.08 & 1.48 & 5.18 & 5.12 & 0.82 & 4.82 & 4.82 \\
\hline & $1 / 20$ & 3500 & 2.85 & 5.67 & 5.43 & 0.85 & 3.77 & 5.08 & 0.73 & 5.18 & 5.12 & 0.20 & 3.09 & 4.82 \\
\hline & $1 / 40$ & 1750 & 0.81 & \begin{tabular}{|l|}
5.67 \\
\end{tabular} & 5.43 & 0.57 & 3.13 & 5.08 & 0.12 & 5.18 & 5.12 & 0.20 & 2.45 & 3.39 \\
\hline & $1 / 80$ & 875 & 0.35 & 2.11 & 5.43 & NT & 1.61 & 2.53 & -0.29 & 1.50 & 2.63 & NT & 0.75 & 1.75 \\
\hline & $1 / 160$ & 438 & NT & 0.63 & 1.15 & NT & 0.57 & 0.58 & NT & 0.11 & 0.96 & NT & -0.28 & 0.62 \\
\hline & $1 / 320$ & 219 & NT & NT & NT & NT & NT & 0.21 & NT & NT & NT & NT & NT & 0.56 \\
\hline Virox 5 & Undiluted & 5000 & 2.67 & \begin{tabular}{|l|}
5.94 \\
\end{tabular} & 5.94 & 1.12 & 5.36 & 5.36 & 1.26 & 4.72 & 5.54 & 0.35 & 3.42 & 5.02 \\
\hline CaviCide & Undiluted & 2800 & 0.92 & \begin{tabular}{|l|}
2.89 \\
\end{tabular} & 3.62 & NT & NT & NT & 0.63 & 2.68 & 3.29 & NT & NT & NT \\
\hline$T^{3} 6$ & Undiluted & 2000 & 0.39 & 1.92 & 2.42 & NT & NT & NT & 1.77 & 2.79 & 2.90 & NT & NT & NT \\
\hline
\end{tabular}

Bold mean values refer to inactivation of the surrogate virus. Experimental trials were performed in triplicate.

NT refers to not tested. 


\section{CHAPTER 4 Conclusion and Recommendations for Future Work}

In summary, several disinfectants including Domestic Miraclean Bleach (sodium hypochlorite) at $5400 \mathrm{ppm}$ after 1 minute (with soil load) or $1350 \mathrm{ppm}$ after 5 minutes (with or without soil load), Accel at $35000 \mathrm{ppm}$ after 10 minutes (with or without soil load), and $\mathrm{T}^{3} 6$ at $2000 \mathrm{ppm}$ after 10 minutes (without soil load) inactivated the virus and produced a $\sim 5.5$ to $6.8 \log _{10}$ reduction in MNV-1 titer. For FCV, disinfectants including Domestic Miraclean Bleach at $2700 \mathrm{ppm}$ after 5 minutes (with soil load) or $1350 \mathrm{ppm}$ after 10 minutes (with or without soil load), Accel at 7000 ppm after 5 minutes (with soil load), 1750 ppm after 5 minutes (without soil load) or $3500 \mathrm{ppm}$ after 10 minutes (with soil load), and Virox 5 at $5000 \mathrm{ppm}$ after 10 minutes (with or without soil load) inactivated the virus, producing a $\sim 4.6$ to $5.9 \log _{10}$ reduction in FCV titer. An unexpected finding was the resistance of MNV-1 towards AHP-based disinfectants and the sensitivity of MNV-1 towards disinfectants containing $70 \%$ ethanol, compared to FCV which showed a completely opposite outcome when exposed to both disinfectants, where FCV was sensitive towards AHP-based products but not products containing $70 \%$ ethanol. Due to the sensitivities of MNV-1 and RAW 264.7 macrophage mouse cells towards alcohol and the lack of a neutralizer, it was difficult to accurately determine MNV-1 survivors after exposure to alcohol. The results presented in this study relating to disinfectants containing alcohol (CaviCide and $\mathrm{T}^{3} 6$ ) and Accel at $35000 \mathrm{ppm}$ only offer an estimate of the MNV-1 viral titer remaining after exposure to the disinfectants. It is important to note that these differences in susceptibility of both surrogate viruses, especially alcohol sensitivity, may lead to different interpretations as to which disinfectants are most effective in killing human norovirus, and because of these differences, it may be important to continue using both surrogate viruses for in vitro testing to provide a more reliable estimate of the effectiveness of disinfectants against human norovirus. 
In particular, the most resistant norovirus surrogate viruses should be used for selected disinfectant products. For example, it may be wise to use MNV-1 as the surrogate virus for disinfectant studies involving AHP-based disinfectants, and FCV for alcohol-based disinfectant studies. Based on these findings, the most effective disinfectant capable of inactivating both surrogates of norovirus on stainless steel surfaces in the absence of a pre-rinsing and/or precleaning step is sodium hypochlorite at concentrations of between 1350 and 5400 ppm and at recommended contact times of between 5 and 10 minutes, depending on the contact time allowed and the amount of soil load present on the environmental surface. As MNV-1 was observed to be more sensitive to disinfectants containing an ethanol content of $70 \%$ than FCV, more studies are needed to provide a better understanding of the efficacy and significance of alcohol-based disinfectants and handrubs against MNV-1, FCV and human norovirus. 


\section{BIBLIOGRAPHY}

Aidaros, H., \& Gaudin, O. G. (1983). A gel filtration method to test the efficiency of virucidal disinfectants. Comparative Immunology, Microbiology and Infectious Diseases, 6, 221-226.

American Type Culture Collection. (2012a). ATCC number CCL-94. Retrieved from http://www.atcc.org/ATCCAdvancedCatalogSearch/ProductDetails/tabid/452/Default.aspx?ATC CNum=CCL-94\&Template=cellBiology on December 8, 2008.

American Type Culture Collection. (2012b). ATCC number TIB-71. Retrieved from http://www.atcc.org/ATCCAdvancedCatalogSearch/ProductDetails/tabid/452/Default.aspx?ATC CNum=TIB-71\&Template=cellBiology on December 8, 2008.

American Type Culture Collection. (2012c). ATCC number VR-782. Retrieved from http://www.atcc.org/ATCCAdvancedCatalogSearch/ProductDetails/tabid/452/Default.aspx?ATC CNum=VR-782\&Template=animalVirology on December 8, 2008.

Association of Official Analytical Chemists. (1975). Official Methods of Analysis of the Association of Official Analytical Chemists (12th ed.). Washington, DC: Association of Official Analytical Chemists.

ASTM International. (1998). ASTM E1482 - 04 standard test method for neutralization of virucidal agents in virucidal efficacy evaluations. West Conshohocken, PA.

Atmar, R. L., Bernstein, D. I., Harro, C. D., Al-Ibrahim, M. S., Chen, W. H., Ferreira, J., Estes, M. K., Graham, D. Y., Opekun, A. R., Richardson, C., \& Mendelman, P. M. (2011). Norovirus vaccine against experimental human Norwalk virus illness. The New England Journal of Medicine, 365, 2178-2187.

Bae, J., \& Schwab, K. J. (2008). Evaluation of murine norovirus, feline calicivirus, poliovirus, and MS2 as surrogates for human norovirus in a model of viral persistence in surface water and groundwater. Applied and Environmental Microbiology, 74, 477-484.

Baert, L., Wobus, C. E., Coillie, E. V., Thackray, L. B., Debevere, J., \& Uyttendaele, M. (2008). Detection of murine norovirus 1 by using plaque assay, transfection assay, and real-time reverse transcription-PCR before and after heat exposure. Applied and Environmental Microbiology, 74, 543-546.

Ball, J. M., Graham, D. Y., Opekun, A. R., Gilger, M. A., Guerrero, R. A., \& Estes, M. K. (1999). Recombinant Norwalk virus-like particles given orally to volunteers: Phase I study. Gastroenterology, 117, 40-48.

Barker, J., Vipond, I. B., \& Bloomfield, S. F. (2004). Effects of cleaning and disinfection in reducing the spread of norovirus contamination via environmental surfaces. Journal of Hospital Infection, 58, 42-49. 
Belliot, G., Lavaux, A., Souihel, D., Agnello, D., \& Pothier, P. (2008). Use of murine norovirus as a surrogate to evaluate resistance of human norovirus to disinfectants. Applied and Environmental Microbiology, 74, 3315-3318.

Bergdoll, M. S. (1989). Staphylococcus aureus. In M. P. Doyle (Ed.), Foodborne Bacterial Pathogens (pp. 463-523). New York, NY: Marcel Dekker, Inc.

Bidawid, S., Farber, J. M., \& Sattar, S. A. (2000). Contamination of foods by food handlers: experiments on hepatitis A virus transfer to food and its interruption. Applied and Environmental Microbiology, 66, 2759-2763.

Bidawid, S., Malik, N., Adegbunrin, O., Sattar, S. A., \& Farber, J. M. (2003). A feline kidney cell line-based plaque assay for feline calicivirus, a surrogate for Norwalk virus. Journal of Virological Methods, 107, 163-167.

Boxman, I., Dijkman, R., Verhoef, L., Maat, A., van Dijk, G., Vennema, H., \& Koopmans, M. (2009). Norovirus on swabs taken from hands illustrate route of transmission: a case study. Journal of Food Protection, 72, 1753-1755.

British Columbia Centre for Disease Control. (2011). Communicable disease control: management of gastrointestinal illness outbreaks in child care facilities, January 2011. Retrieved from http://www.bccdc.ca/NR/rdonlyres/E6320AA6-FEC7-42AC-840B3721A106FE80/0/CCFOutbreakguidelines.pdf on July 12, 2012.

Calderon-Margalit, R., Sheffer, R., Halperin, T., Orr, N., Cohen, D., \& Shohat, T. (2005). A large-scale gastroenteritis outbreak associated with norovirus in nursing homes. Epidemiology and Infection, 133, 35-40.

Cannon, J. L., Papafragkou, E., Park, G. W., Osborne, J., Jaykus, L.-A., \& Vinjé, J. (2006). Surrogates for the study of norovirus stability and inactivation in the environment: a comparison of murine norovirus and feline calicivirus. Journal of Food Protection, 69, 2761-2765.

Carter, M. J., Milton, I. D., Meanger, J., Bennett, M., Gaskell, R. M., \& Turner, P. C. (1992). The complete nucleotide sequence of a feline calicivirus. Virology, 190, 443-448.

Caul, E. O. (1994). Small round structured viruses: airborne transmission and hospital control. The Lancet, 343, 1240-1242.

Centers for Disease Control and Prevention. (2010). Surveillance for foodborne disease outbreaks - United States, 2007. Morbidity and Mortality Weekly Report, 59, 973-979. Retrieved from http://www.cdc.gov/mmwr/pdf/wk/mm5931.pdf on December 30, 2012.

Centers for Disease Control and Prevention. (2011a). CDC estimates of foodborne illness in the United States. Retrieved from http://www.cdc.gov/foodborneburden/2011-foodborneestimates.html on August 5, 2012. 
Centers for Disease Control and Prevention. (2011b). Guideline for the prevention and control of norovirus gastroenteritis outbreaks in healthcare settings. Retrieved from http://www.cdc.gov/hicpac/pdf/norovirus/Norovirus-Guideline-2011.pdf on February 23, 2012.

Centers for Disease Control and Prevention. (2011c). Updated norovirus outbreak management and disease prevention guidelines. Morbidity and Mortality Weekly Report, 60, 1-15. Retrieved from http://www.cdc.gov/mmwr/pdf/rr/rr6003.pdf on February 23, 2012.

Chadwick, P. R., Beards, G., Brown, D., Caul, E. O., Cheesbrough, J., Clarke, I., Curry, A., O'Brien, S., Quigley, K., Sellwood, J., \& Westmoreland, D. (2000). Management of hospital outbreaks of gastro-enteritis due to small round structured viruses. Journal of Hospital Infection, $45,1-10$.

Cheesbrough, J. S., Green, J., Gallimore, C. I., Wright, P. A., \& Brown, D. W. G. (2000). Widespread environmental contamination with Norwalk-like viruses (NLV) detected in a prolonged hotel outbreak of gastroenteritis. Epidemiology and Infection, 125, 93-98.

Cheng, P. K. C., Wong, D. K. K., Chung, T. W. H., \& Lim, W. W. L. (2005). Norovirus contamination found in oysters worldwide. Journal of Medical Virology, 76, 593-597.

Clay, S., Maherchandani, S., Malik, Y. S., \& Goyal, S. M. (2006). Survival on uncommon fomites of feline calicivirus, a surrogate of noroviruses. American Journal of Infection Control, 34, 41-43.

Conner, D. E., \& Kotrola, J. S. (1995). Growth and survival of Escherichia coli O157:H7 under acidic conditions. Applied and Environmental Microbiology, 61, 382-385.

Coyne, K. P., Reed, F. C., Porter, C. J., Dawson, S., Gaskell, R. M., \& Radford, A. D. (2006). Recombination of feline calicivirus within an endemically infected cat colony. Journal of General Virology, 87, 921-926.

Denyer, S. P., \& Stewart, G. S. A. B. (1998). Mechanisms of action of disinfectants. International Biodeterioration and Biodegradation, 41, 261-268.

Donaldson, E. F., Lindesmith, L. C., LoBue, A. D., \& Baric, R. S. (2010). Viral shape-shifting: norovirus evasion of the human immune system. Nature Reviews Microbiology, 8, 231-241.

Doultree, J. C., Druce, J. D., Birch, C. J., Bowden, D. S., \& Marshall, J. A. (1999). Inactivation of feline calicivirus, a Norwalk virus surrogate. Journal of Hospital Infection, 41, 51-57.

Dourou, D., Beauchamp, C. S., Yoon, Y., Geornaras, I., Belk, K. E., Smith, G. C., Nychas, G.-J. E., \& Sofos, J. N. (2011). Attachment and biofilm formation by Escherichia coli O157:H7 at different temperatures, on various food-contact surfaces encountered in beef processing. International Journal of Food Microbiology, 149, 262-268. 
Duffy, G. (2006). Emerging pathogenic E. coli. In Motarjemi, Y., \& Adams, M. (Ed.), Emerging foodborne pathogens (pp. 253-281). Cambridge, England: Woodhead Publishing Limited.

Duizer, E., Schwab, K. J., Neill, F. H., Atmar, R. L., Koopmans, M. P. G., \& Estes, M. K. (2004). Laboratory efforts to cultivate noroviruses. Journal of General Virology, 85, 79-87.

Estes, M. K., Ball, J. M., Guerrero, R. A., Opekun, A. R., Gilger, M. A., Pacheco, S. S., \& Graham, D. Y. (2000). Norwalk virus vaccines: challenges and progress. The Journal of Infectious Diseases, 181, S367-S373.

Estes, M. K., Prasad, B. V. V., \& Atmar, R. L. (2006). Noroviruses everywhere: has something changed? Current Opinion in Infectious Diseases, 19, 467-474.

Fastier, L. B. (1957). A new feline virus isolated in tissue culture. American Journal of Veterinary Research, 67, 382-389.

Frank, J. F., \& Chmielewski, R. A. N. (1997). Effectiveness of sanitation with quaternary ammonium compound or chlorine on stainless steel and other domestic food-preparation surfaces. Journal of Food Protection, 60, 43-47.

Fretz, R., Svoboda, P., Lüthi, T. M., Tanner, M., \& Baumgartner, A. (2005). Outbreaks of gastroenteritis due to infections with norovirus in Switzerland, 2001-2003. Epidemiology \& Infection, 133, 429-437.

Fuster-Valls, N., Hernández-Herrero, M., Marín-de-Mateo, M., \& Rodríguez-Jerez, J. J. (2008). Effect of different environmental conditions on the bacteria survival on stainless steel surfaces. Food Control, 19, 308-314.

Ganguli, P. S., Chen, W., \& Yates, M. V. (2011). Detection of murine norovirus-1 by using TAT peptide-delivered molecular beacons. Applied and Environmental Microbiology, 77, 5517-5520.

Garcia, L. S. (Eds.). (2010). Clinical Microbiology Procedures Handbook (3rd ed.). Washington, DC: American Society for Microbiology.

Gehrke, C., Steinmann, J., \& Goroncy-Bermes, P. (2004). Inactivation of feline calicivirus, a surrogate of norovirus (formerly Norwalk-like viruses), by different types of alcohol in vitro and in vivo. Journal of Hospital Infection, 56, 49-55.

Gélinas, P., \& Goulet, J. (1983). Neutralization of the activity of eight disinfectants by organic matter. Journal of Applied Bacteriology, 54, 243-247.

Gibson, K. E., \& Schwab, K. J. (2011). Thermal inactivation of human norovirus surrogates. Food and Environmental Virology, 3, 74-77.

Gibson, K. E., Crandall, P. G., \& Ricke, S. C. (2012). Removal and transfer of viruses on food contact surfaces by cleaning cloths. Applied and Environmental Microbiology, 78, 3037-3044. 
Girard, M., Ngazoa, S., Mattison, K., \& Jean, J. (2010). Attachment of noroviruses to stainless steel and their inactivation, using household disinfectants. Journal of Food Protection, 73, 400404.

Gonin, P., Couillard, M., \& d'Halewyn, M.-A. (2000). Genetic diversity and molecular epidemiology of Norwalk-like viruses. The Journal of Infectious Diseases, 182, 691-697.

Goodridge, L., Goodridge, C., Wu, J., Griffiths, M., \& Pawliszyn, J. (2004). Isoelectric point determination of norovirus virus-like particles by capillary isoelectric focusing with whole column imaging detection. Analytical Chemistry, 76, 48-52.

Green, K. Y., Ando, T., Balayan, M. S., Berke, T., Clarke, I. N., Estes, M. K., Matson, D. O., Nakata, S., Neill, J. D., Studdert, M. J., \& Thiel, H.-J. (2000). Taxonomy of the caliciviruses. The Journal of Infectious Diseases, 181, S322-S330.

Grist, N. R., Bell, E. J., Follett, E. A. C., \& Urquhart, G. E. D. (1979). Diagnostic Methods in Clinical Virology (3rd ed.). Oxford, England: Blackwell Scientific Publications.

Hardy, M. E. (2005). Norovirus protein structure and function. FEMS Microbiology Letters, 253, $1-8$.

Harris, J. P., Edmunds, W. J., Pebody, R., Brown, D. W., \& Lopman, B. A. (2008). Deaths from norovirus among the elderly, England and Wales. Emerging Infectious Diseases, 14, 1546-1552.

Health Canada. (2010). Concentration of Norovirus Genogroups I and II from Contaminated Oysters and their Detection using the Reverse-Transcriptase Polymerase Chain Reaction (Laboratory Procedure OPFLP-01). Retrieved from http://www.hc-sc.gc.ca/fn-an/res-rech/analymeth/microbio/volume5/opflp_01-eng.php on February 6, 2011.

Horm, K. M., \& D’Souza, D. H. (2011). Survival of human norovirus surrogates in milk, orange, and pomegranate juice, and juice blends at refrigeration $\left(4^{\circ} \mathrm{C}\right)$. Food Microbiology, 28, 10541061.

Hota, B. (2004). Contamination, disinfection, and cross-colonization: are hospital surfaces reservoirs for nosocomial infection? Clinical Infectious Diseases, 39, 1182-1189.

Hsu, C. C., Riley, L. K., \& Livingston, R. S. (2007). Molecular characterization of three novel murine noroviruses. Virus Genes, 34, 147-155.

Hudson, J. B., Sharma, M., \& Petric, M. (2007). Inactivation of norovirus by ozone gas in conditions relevant to healthcare. Journal of Hospital Infection, 66, 40-45.

Hurley, K. E., Pesavento, P. A., Pedersen, N. C., Poland, A. M., Wilson, E., \& Foley, J. E. (2004). An outbreak of virulent systemic feline calicivirus disease. Journal of the American Veterinary Medical Association, 224, 241-249. 
Hutson, A. M., Atmar, R. L., Graham, D. Y., \& Estes, M. K. (2002). Norwalk virus infection and disease is associated with ABO histo-blood group type. The Journal of Infectious Diseases, 185, 1335-1337.

Hutson, A. M., Atmar, R. L., \& Estes, M. K. (2004). Norovirus disease: changing epidemiology and host susceptibility factors. Trends in Microbiology, 12, 279-287.

Isakbaeva, E. T., Bulens, S. N., Beard, R. S., Adams, S., Monroe, S. S., Chaves, S. S., Widdowson, M.-A., \& Glass, R. I. (2005). Norovirus and child care: challenges in outbreak control. The Pediatric Infectious Disease Journal, 24, 561-563.

Jimenez, L., \& Chiang, M. (2006). Virucidal activity of a quaternary ammonium compound disinfectant against feline calicivirus: a surrogate for norovirus. American Journal of Infection Control, 34, 269-273.

Kageyama, T., Kojima, S., Shinohara, M., Uchida, K., Fukushi, S., Hoshino, F. B., Takeda, N., \& Katayama, K. (2003). Broadly reactive and highly sensitive assay for Norwalk-like viruses based on real-time quantitative reverse transcription-PCR. Journal of Clinical Microbiology, 41, 1548-1557.

Kapikian, A. Z., Wyatt, R. G., Dolin, R., Thornhill, T. S., Kalica, A. R., \& Chanock, R. M. (1972). Visualization by immune election microscopy of a 27-nm particle associated with acute infectious nonbacterial gastroenteritis. Journal of Virology, 10, 1075-1081.

Karst, S. M. (2010). Pathogenesis of noroviruses, emerging RNA viruses. Viruses, 2, 748-781.

Katayama, K., Shirato-Horikoshi, H., Kojima, S., Kageyama, T., Oka, T., Hoshino, F. B., Fukushi, S., Shinohara, M., Uchida, K., Suzuki, Y., Gojobori, T., \& Takeda, N. (2002). Phylogenetic analysis of the complete genome of 18 Norwalk-like viruses. Virology, 299, 225239.

Kojima, S., Kageyama, T., Fukushi, S., Hoshino, F. B., Shinohara, M., Uchida, K., Natori, K., Takeda, N., \& Katayama, K. (2002). Genogroup-specific PCR primers for detection of Norwalklike viruses. Journal of Virological Methods, 100, 107-114.

Kreutz, L. C., \& Seal, B. S. (1995). The pathway of feline calicivirus entry. Virus Research, 35, 63-70.

Kroneman, A., Harris, J., Vennema, H., Duizer, E., van Duynhoven, Y., Gray, J., Iturriza, M., Böttiger, B., Falkenhorst, G., Johnsen, C., von Bonsdorff, C.-H., Maunula, L., Kuusi, M., Pothier, P., Gallay, A., Schreier, E., Koch, J., Szücs, G., Reuter, G., Krisztalovics, K., Lynch, M., McKeown, P., Foley, B., Coughlan, S., Ruggeri, F. M., Di Bartolo, I., Vainio, K., Isakbaeva, E., Poljsak-Prijatelj, M., Hocevar Grom, A., Bosch, A., Buesa, J., Sanchez Fauquier, A., HernandézPezzi, G., Hedlund, K.-O., \& Koopmans, M. (2007). Data quality of 5 years of central norovirus outbreak reporting in the European Network for food-borne viruses. Journal of Public Health, $30,82-90$. 
Lages, S. L. S., Ramakrishnan, M. A., \& Goyal, S. M. (2008). In-vivo efficacy of hand sanitizers against feline calicivirus: a surrogate for norovirus. Journal of Hospital Infection, 68, 159-163.

Lamhoujeb, S., Fliss, I., Ngazoa, S. E., Jean, J. (2009). Molecular study of the persistence of infectious human norovirus on food-contact surfaces. Food and Environmental Virology, 1, 5156.

Lay, M. K., Atmar, R. L., Guix, S., Bharadwaj, U., He, H., Neill, F. H., Sastry, K. J., Yao, Q., \& Estes, M. K. (2010). Norwalk virus does not replicate in human macrophages or dendritic cells derived from the peripheral blood of susceptible humans. Virology, 406, 1-11.

Lees, D. (2000). Viruses and bivalve shellfish. International Journal of Food Microbiology, 59, 81-116.

Lindesmith, L., Moe, C., LePendu, J., Frelinger, J. A., Treanor, J., \& Baric, R. S. (2005). Cellular and humoral immunity following Snow Mountain Virus challenge. Journal of Virology, 79, 2900-2909.

Liu, P., Chien, Y.-W., Papafragkou, E., Hsiao, H.-M., Jaykus, L.-A., \& Moe, C. (2009). Persistence of human noroviruses on food preparation surfaces and human hands. Food and Environmental Virology, 1, 141-147.

Liu, P., Yuen, Y., Hsiao, H.-M., Jaykus, L.-A., \& Moe, C. (2010). Effectiveness of liquid soap and hand sanitizer against Norwalk virus on contaminated hands. Applied and Environmental Microbiology, 76, 394-399.

Lopman, B. A., Reacher, M. H., van Duijnhoven, Y., Hanon, F.-X., Brown, D., \& Koopmans, M. (2003). Viral gastroenteritis outbreaks in Europe, 1995-2000. Emerging Infectious Diseases, 9 , 90-96.

Lopman, B. A., Reacher, M. H., Vipond, I. B., Sarangi, J., \& Brown, D. W. G. (2004). Clinical manifestation of norovirus gastroenteritis in health care settings. Clinical Infectious Diseases, 39, 318-324.

MacCannell, T., Umscheid, C. A., Agarwal, R. K., Lee, I., Kuntz, G., \& Stevenson, K. B. (2011). Guideline for the prevention and control of norovirus gastroenteritis outbreaks in healthcare settings. Infection Control and Hospital Epidemiology, 32, 939-969.

Magulski, T., Paulmann, D., Bischoff, B., Becker, B., Steinmann, E., Steinmann, J., GoroncyBermes, P., \& Steinmann, J. (2009). Inactivation of murine norovirus by chemical biocides on stainless steel. BMC Infectious Diseases, 9, 107.

Maillard, J.-Y. (2001). Virus susceptibility to biocides: an understanding. Reviews in Medical Microbiology, 12, 63-74. 
Malek, M., Barzilay, E., Kramer, A., Camp, B., Jaykus, L.-A., Escudero-Abarca, B., Derrick, G., White, P., Gerba, C., Higgins, C., Vinje, J., Glass, R., Lynch, M., \& Widdowson, M.-A. (2009). Outbreak of norovirus infection among river rafters associated with packaged delicatessen meat, Grand Canyon, 2005. Clinical Infectious Diseases, 48, 31-37.

Malik, Y. S., Maherchandani, S., \& Goyal, S. M. (2006). Comparative efficacy of ethanol and isopropanol against feline calicivirus, a norovirus surrogate. American Journal of Infection Control, 34, 31-35.

Marionneau, S., Ruvoën, N., Le Moullac-Vaidye, B., Clement, M., Cailleau-Thomas, A., RuizPalacois, G., Huang, P., Jiang, X., \& Le Pendu, J. (2002). Norwalk virus binds to histo-blood group antigens present on gastroduodenal epithelial cells of secretor individuals. Gastroenterology, 122, 1967-1977.

Marriott, N. G., \& Gravani, R. B. (2006). Principles of Food Sanitation (5th ed.). New York, New York: Springer.

Marshall, J. A., \& Bruggink, L. D. (2011). The dynamics of norovirus outbreak epidemics: recent insights. International Journal of Environmental Research and Public Health, 8, 11411149.

Matsui, S. M., \& Greenberg, H. B. (2000). Immunity to calicivirus infection. The Journal of Infectious Diseases, 181, S331-S335.

Mattison, K., Karthikeyan, K., Abebe, M., Malik, N., Sattar, S. A., Farber, J. M., \& Bidawid, S. (2007). Survival of calicivirus in foods and on surfaces: experiments with feline calicivirus as a surrogate for norovirus. Journal of Food Protection, 70, 500-503.

Mattison, K., Grudeski, E., Auk, B., Charest, H., Drews, S. J., Fritzinger, A., Gregoricus, N., Hayward, S., Houde, A., Lee, B. E., Pang, X. L., Wong, J., Booth, T. F., \& Vinjé, J. (2009). Multicenter comparison of two norovirus ORF2-based genotyping protocols. Journal of Clinical Microbiology, 47, 3927-3932.

Mattner, F., Sohr, D., Heim, A., Gastmeier, P., Vennema, H., \& Koopmans, M. (2006). Risk groups for clinical complications of norovirus infections: an outbreak investigation. Clinical Microbiology and Infection, 12, 69-74.

Mbithi, J. N., Springthorpe, S., \& Sattar, S. A. (1991). Effect of relative humidity and air temperature on survival of hepatitis A virus on environmental surfaces. Applied and Environmental Microbiology, 57, 1394-1399.

McBain, A. J., Ledder, R. G., Moore, L. E., Catrenich, C. E., \& Gilbert, P. (2004). Effects of quaternary-ammonium-based formulations on bacterial community dynamics and antimicrobial susceptibility. Applied and Environmental Microbiology, 70, 3449-3456. 
McDonnell, G., \& Russell, A. D. (1999). Antiseptics and disinfectants: activity, action, and resistance. Clinical Microbiology Reviews, 12, 147-179.

Moe, C. L. (2009). Preventing norovirus transmission: how should we handle food handlers? Clinical Infectious Diseases, 48, 38-40.

Mounts, A. W., Ando, T., Koopmans, M., Bresee, J. S., Noel, J., \& Glass, R. I. (2000). Cold weather seasonality of gastroenteritis associated with Norwalk-like viruses. The Journal of Infectious Diseases, 181, S284-287.

Neely, A. N., \& Maley, M. P. (2000). Survival of enterococci and staphylococci on hospital fabrics and plastic. Journal of Clinical Microbiology, 38, 724-726.

Neill, J. D., \& Mengeling, W. L. (1988). Further characterization of the virus-specific RNAs in feline calicivirus infected cells. Virus Research, 11, 59-72.

Neill, J. D., Reardon, I. M., \& Heinrikson, R. L. (1991). Nucleotide sequence and expression of the capsid protein gene of feline calicivirus. Journal of Virology, 65, 5440-5447.

Neill, J. D. (2002). The subgenomic RNA of feline calicivirus is packaged into viral particles during infection. Virus Research, 87, 89-93.

Nuanualsuwan, S., \& Cliver, D. O. (2002). Pretreatment to avoid positive RT-PCR results with inactivated viruses. Journal of Virological Methods, 104, 217-225.

Nuermberger, E. (2005). Current issues in the diagnosis, evaluation, and management of gastrointestinal infections. Advanced Studies in Medicine, 5, 90-97.

Omidbakhsh, N., \& Sattar, S. A. (2006). Broad-spectrum microbicidal activity, toxicologic assessment, and materials compatibility of a new generation of accelerated hydrogen peroxidebased environmental surface disinfectant. American Journal of Infection Control, 34, 251-257.

Ormerod, E., \& Jarrett, O. (1978). A classification of feline calicivirus isolates based on plaque morphology. Journal of General Virology, 39, 537-540.

Ossiboff, R. J., Sheh, A., Shotton, J., Pesavento, P. A., \& Parker, J. S. L. (2007). Feline caliciviruses (FCVs) isolated from cats with virulent systemic disease possess in vitro phenotypes distinct from those of other FCV isolates. Journal of General Virology, 88, 506-517.

Pang, X. L., Preiksaitis, J. K., \& Lee, B. (2005). Multiplex real-time RT-PCR for the detection and quantitation of norovirus genogroups I and II in patients with acute gastroenteritis. Journal of Clinical Virology, 33, 168-171.

Park, G. W., Barclay, L., Macinga, D., Charbonneau, D., Pettigrew, C. A., \& Vinjé, J. (2010). Comparative efficacy of seven hand sanitizers against murine norovirus, feline calicivirus, and GII.4 norovirus. Journal of Food Protection, 73, 2232-2238. 
Parrino, T. A., Schreiber, D. S., Trier, J. S., Kapikian, A. Z., \& Blacklow, N. R. (1977). Clinical immunity in acute gastroenteritis caused by Norwalk agent. The New England Journal of Medicine, 297, 86-89.

Patel, M. M., Hall, A. J., Vinjé, J., \& Parashar, U. D. (2009). Noroviruses: a comprehensive review. Journal of Clinical Virology, 44, 1-8.

Pesavento, P. A., MacLachlan, N. J., Dillard-Telm, L., Grant, C. K., \& Hurley, K. F. (2004). Pathologic, immunohistochemical, and electron microscopic findings in naturally occurring virulent systemic feline calicivirus infection in cats. Veterinary Pathology, 41, 257-263.

Poschetto, L. F., Ike, A., Papp, T., Mohn, U., Böhm, R., \& Marschang, R. E. (2007). Comparison of the sensitivities of noroviruses and feline calicivirus to chemical disinfection under field-like conditions. Applied and Environmental Microbiology, 73, 5494-5500.

Prasad, B. V. V., Hardy, M. E., Dokland, T., Bella, J., Rossmann, M. G., \& Estes, M. K. (1999). X-ray crystallographic structure of the Norwalk virus capsid. Science, 286, 287-290.

Public Health Agency of Canada (2005). Noroviruses - fact sheet. Retrieved from http://www.phac-aspc.gc.ca/id-mi/norovirus-eng.php on December 1, 2010.

Public Health Agency of Canada (2010). 2010 annual summary of laboratory surveillance data. Retrieved from http://www.nml-lnm.gc.ca/NESP-PNSME/assets/pdf/NESP_2010_Annual_ Report_ENG.pdf on April 9, 2012.

Radford, A. D., Gaskell, R. M., \& Hart, C. A. (2004). Human norovirus infection and the lessons from animal caliciviruses. Current Opinion in Infectious Diseases, 17, 471-478.

Radford, A. D., Dawson, S., Coyne, K. P., Porter, C. J., \& Gaskell, R. M. (2006). The challenge for the next generation of feline calicivirus vaccines. Veterinary Microbiology, 117, 14-18.

Raschke, W. C., Baird, S., Ralph, P., \& Nakoinz, I. (1978). Functional macrophage cell lines transformed by Abelson leukemia virus. Cell, 15, 261-267.

Richards, G. P. (1999). Limitations of molecular biological techniques for assessing the virological safety of foods. Journal of Food Protection, 62, 691-697.

Rutala, W. A., \& Weber, D. J. (1997). Uses of inorganic hypochlorite (bleach) in health-care facilities. Clinical Microbiology Reviews, 10, 597-610.

Rutala, W. A., Peacock, J. E., Gergen, M. F., Sobsey, M. D., \& Weber, D. J. (2006). Efficacy of hospital germicides against adenovirus 8, a common cause of epidemic keratoconjunctivitis in health care facilities. Antimicrobial Agents and Chemotherapy, 50, 1419-1424. 
Sato, Y., Ohe, K., Murakami, M., Fukuyama, M., Furuhata, K., Kishikawa, S., Suzuki, Y., Kiuchi, A., Hara, M., Ishikawa, Y., \& Taneno, A. (2002). Phylogenetic analysis of field isolates of feline calicivirus (FCV) in Japan by sequencing part of its capsid gene. Veterinary Research Communications, 26, 205-219.

Sattar, S. A., Tetro, J., Bidawid, S., \& Farber, J. (2000). Foodborne spread of hepatitis A: recent studies on virus survival, transfer and inactivation. Canadian Journal of Infectious Diseases, 11, 159-163.

Sattar, S. A., Springthorpe, V. S., Adegbunrin, O., Zafer, A. A., \& Busa, M. (2003). A disc-based quantitative carrier test to assess the virucidal activity of chemical germicides. Journal of Virological Methods, 112, 3-12.

Sattar, S. A., Ali, M., \& Tetro, J. A. (2011). In vivo comparison of two human norovirus surrogates for testing ethanol-based handrubs: the mouse chasing the cat! PLoS ONE, 6, e17340.

Scallan, E., Hoekstra, R. M., Angulo, F. J., Tauxe, R. V., Widdowson, M.-A., Roy, S. L., Jones, J. L., \& Griffin, P. M. (2011). Food illness acquired in the United States - major pathogens. Emerging Infectious Diseases, 17, 7-15.

Schmidt, N. J., \& Emmons, R. W. (Eds.). (1989). Diagnostic procedures for viral, rickettsial and chlamydial infections (6th ed.). Washington, DC: American Public Health Association, Inc.

Schmitt, M., Schuler-Schmid, U., \& Schmidt-Lorenz, W. (1990). Temperature limits of growth, TNase and enterotoxin production of Staphylococcus aureus strains isolated from foods. International Journal of Food Microbiology, 11, 1-20.

Schreiber, D. S., Blacklow, N. R., \& Trier, J. S. (1974). The small intestinal lesion induced by Hawaii agent acute infectious nonbacterial gastroenteritis. The Journal of Infectious Diseases, 129, 705-708.

Seal, B. S., Ridpath, J. F., \& Mengeling, W. L. (1993). Analysis of feline calicivirus capsid protein genes: identification of variable antigenic determinant regions of the protein. Journal of General Virology, 74, 2519-2524.

Seo, K., Lee, J. E., Lim, M. Y., \& Ko, G. (2012). Effect of temperature, pH, and NaCl on the inactivation kinetics of murine norovirus. Journal of Food Protection, 75, 533-540.

Steinmann, J. (2004). Surrogate viruses for testing virucidal efficacy of chemical disinfectants. Journal of Hospital Infection, 56, S49-S54.

Straub, T. M., Bartholomew, R. A., Valdez, C. O., Valentine, N. B., Dohnalkova, A., Ozanich, R. M., Bruckner-Lea, C. J., \& Call, D. R. (2011). Human norovirus infection of caco-2 cells grown as a 3-dimensional tissue structure. Journal of Water and Health, 9, 225-240. 
Takahashi, H., Ohuchi, A., Miya, S., Izawa, Y., \& Kimura, B. (2011). Effect of food residues on norovirus survival on stainless steel surfaces. PLOS ONE, 6, e21951.

Thorven, M., Grahn, A., Hedlund, K.-O., Johansson, H., Wahlfrid, C., Larson, G., \& Svensson, L. (2005). A homozygous nonsense mutation $(428 \mathrm{G} \rightarrow \mathrm{A})$ in the human secretor (FUT2) gene provides resistance to symptomatic norovirus (GGII) infections. Journal of Virology, 79, 1535115355.

Tian, P., Engelbrektson, A. L., Jiang, X., Zhong, W., \& Mandrell, R. E. (2007). Norovirus recognizes histo-blood group antigens on gastrointestinal cells of clams, mussels, and oysters: a possible mechanism of bioaccumulation. Journal of Food Protection, 70, 2140-2147.

Topping, J. R., Schnerr, H., Haines, J., Scott, M., Carter, M. J., Willcocks, M. M., Bellamy, K., Brown, D. W., Gray, J. J., Gallimore, C. I., \& Knight, A. I. (2009). Temperature inactivation of feline calicivirus vaccine strain FCV F-9 in comparison with human noroviruses using an RNA exposure assay and reverse transcribed quantitative real-time polymerase chain reaction - a novel method for predicting virus infectivity. Journal of Virological Methods, 156, 89-95.

Trujillo, A. A., McCaustland, K. A., Zheng, D.-P., Hadley, L. A., Vaughn, G., Adams, S. M., Ando, T., Glass, R. I., \& Monroe, S. S. (2006). Use of TaqMan real-time reverse transcriptionPCR for rapid detection, quantification, and typing of norovirus. Journal of Clinical

Microbiology, 44, 1405-1412.

United States Environmental Protection Agency. (2009). List G: EPA's registered antimicrobial products effective against norovirus (Norwalk-like virus). Retrieved from http://www.epa.gov/oppad001/list_g_norovirus.pdf on July 12, 2012.

United States Food and Drug Administration. (2012). Guidance for industry and Food and Drug Administration staff - class II special controls guidance document: norovirus serological reagents. Retrieved from

http://www.fda.gov/downloads/MedicalDevices/DeviceRegulationandGuidance/ GuidanceDocuments/UCM295090.pdf on February 24, 2013.

Urakami, H., Ikarashi, K., Okamoto, K., Abe, Y., Ikarashi, T., Kono, T., Konagaya, Y., \& Tanaka, N. (2007). Chlorine sensitivity of feline calicivirus, a norovirus surrogate. Applied and Environmental Microbiology, 73, 5679-5682.

Van Elsas, J. D., Semenov, A. V., Costa, R., \& Trevors, J. T. (2011). Survival of Escherichia coli in the environment: fundamental and public health aspects. The ISME Journal, 5, 173-183.

Vashist, S., Bailey, D., Putics, A., \& Goodfellow, I. (2009). Model systems for the study of human norovirus biology. Future Virology, 4, 353-367.

Verhoef, L., Vennema, H., van Pelt, W., Lees, D., Boshuizen, H., Henshilwood, K., \& Koopmans, M. (2010). Use of norovirus genotype profiles to differentiate origins of foodborne outbreaks. Emerging Infectious Diseases, 16, 617-624. 
Vinjé, J., Vennema, H., Maunula, L., von Bonsdorff, C.-H., Hoehne, M., Schreier, E., Richards, A., Green, J., Brown, D., Beard, S. S., Monroe, S. S., de Bruin, E., Svensson, L., \& Koopmans, M. P. G. (2003). International collaborative study to compare reverse transcriptase PCR assays for detection and genotyping of noroviruses. Journal of Clinical Microbiology, 41, 1423-1433.

Vinjé, J., Hamidjaja, R. A., \& Sobsey, M. D. (2004). Development and application of a capsid VP1 (region D) based reverse transcription PCR assay for genotyping of genogroup I and II noroviruses. Journal of Virological Methods, 116, 109-117.

Virox Technologies Inc. (2011). Accelerated Hydrogen Peroxide (AHP) Frequently Asked Questions. Retrieved from http://www.virox.com/ahp/faq/default.aspx on April 8, 2012.

Weber, D. J., Rutala, W. A., Miller, M. B., Huslage, K., \& Sickbert-Bennett, E. (2010). Role of hospital surfaces in the transmission of emerging health care-associated pathogens: norovirus, Clostridium difficile, and Acinetobacter species. American Journal of Infection Control, 38, S25S33.

Whitehead, K., \& McCue, K. A. (2010). Virucidal efficacy of disinfectant actives against feline calicivirus, a surrogate for norovirus, in a short contact time. American Journal of Infection Control, 38, 26-30.

Widdowson, M.-A., Cramer, E. H., Hadley, L., Bresee, J. S., Beard, S., Bulens, S. N., Charles, M., Chege, W., Isakbaeva, E., Wright, J. G., Mintz, E., Forney, D., Massey, J., Glass, R. I., \& Monroe, S. S. (2004). Outbreaks of acute gastroenteritis on cruise ships and on land: identification of a predominant circulating strain of noroviruses - United States, 2002. The Journal of Infectious Diseases, 190, 27-36.

Widdowson, M.-A., Sulka, A., Bulens, S. N., Beard, S., Chaves, S. S., Hammond, R., Salehi, E. D. P., Swanson, E., Totaro, J., Woron, R., Mead, P. S., Bresee, J. S., Monroe, S. S., \& Glass, R. I. (2005). Norovirus and foodborne disease, United States, 1991-2000. Emerging Infectious Diseases, 11, 95-102.

Wikswo, M. E., Cortes, J., Hall, A. J., Vaughan, G., Howard, C., Gregoricus, N., \& Cramer, E. H. (2011). Disease transmission and passenger behaviors during a high morbidity norovirus outbreak on a cruise ship, January 2009. Clinical Infectious Diseases, 52, 1116-1122.

Wobus, C. E., Karst, S. M., Thackray, L. B., Chang, K.-O., Sosnovtsev, S. V., Belliot, G., Krug, A., Mackenzie, J. M., Green, K. Y., \& Virgin, H. W. (2004). Replication of norovirus in cell culture reveals a tropism for dendritic cells and macrophages. PLoS Biology, 2, 2076-2084.

Wu, H. M., Fornek, M., Schwab, K. J., Chapin, A. R., Gibson, K., Schwab, E., Spencer, C., \& Henning, K. (2005). A norovirus outbreak at a long-term care facility: the role of environmental surface contamination. Infection Control and Hospital Epidemiology, 26, 802-810. 
Wyatt, R. G., Dolin, R., Blacklow, N. R., DuPont, H. L., Buscho, R. F., Thornhill, T. S., Kapikian, A. Z., \& Chanock, R. M. (1974). Comparison of three agents of acute infectious nonbacterial gastroenteritis by cross-challenge in volunteers. Journal of Infectious Diseases, 129, 709-714.

Yamada, K., Ogawa, H., Hara, A., Yoshida, Y., Yonezawa, Y., Karibe, K., Nghia, V. B., Yoshimura, H., Yamamoto, Y., Yamada, M., Nakamura, K., \& Imai, K. (2009). Mechanism of the antiviral effect of hydroxytyrosol on influenza virus appears to involve morphological change of the virus. Antiviral Research, 83, 35-44.

Yuk, H.-G., \& Marshall, D. L. (2004). Adaption of Escherichia coli O157:H7 to pH alters membrane lipid composition, verotoxin secretion and resistance to simulated gastric fluid acid. Applied and Environmental Microbiology, 70, 3500-3505.

Zheng, D.-P., Widdowson, M.-A., Glass, R. I., \& Vinjé, J. (2010). Molecular epidemiology of genogroup II-genotype 4 noroviruses in the United States between 1994 and 2006. Journal of Clinical Microbiology, 48, 168-177.

Zingg, W., Colombo, C., Jucker, T., Bossart, W., \& Ruef, C. (2005). Impact of an outbreak of norovirus infection on hospital resources. Infection Control and Hospital Epidemiology, 26, 263267. 


\section{APPENDICES}

\section{Appendix A. Solution and Media Preparation}

Table A1. Preparation of sodium thiosulphate and letheen broth neutralizer solutions.

\begin{tabular}{|l|l|l|l|}
\hline Neutralizer & Volume & Ingredients & Additional reagents \\
\hline Sodium thiosulphate & $\begin{array}{l}500 \mathrm{~mL} \text { distilled, } \\
\text { deionized water } \\
\text { thiosulphate } \\
\text { pentahydrate }\end{array}$ & $\begin{array}{l}5 \mathrm{~mL} \text { Fungizone }{ }^{\circledR} \\
\text { amphotericin } \mathrm{B}, \\
2.5 \mathrm{~mL} \text { gentamicin } \\
\text { reagent solution, } 5 \mathrm{~mL} \\
\text { penicillin } \mathrm{G} \text { potassium } \\
\text { salt }\end{array}$ \\
\hline Letheen broth & $\begin{array}{l}500 \mathrm{~mL} \text { distilled, } \\
\text { deionized water }\end{array}$ & $\begin{array}{l}\text { 51.4 g letheen broth, } \\
10 \mathrm{~g} \text { sodium } \\
\text { thiosulphate } \\
\text { pentahydrate }\end{array}$ & $\begin{array}{l}5 \mathrm{~mL} \text { Fungizone }{ }^{\circledR} \\
\text { amphotericin } \mathrm{B}, \\
2.5 \mathrm{~mL} \text { gentamicin } \\
\text { reagent solution, } 5 \mathrm{~mL} \\
\text { penicillin G potassium } \\
\text { salt }\end{array}$ \\
\hline
\end{tabular}

Table A2. Preparation of supplemented alpha-MEM and 2X alpha-MEM growth medium.

\begin{tabular}{|c|c|c|c|}
\hline Growth medium & \begin{tabular}{|l} 
Volume $(\mathrm{mL})$ \\
\end{tabular} & Ingredients & $\begin{array}{l}\text { Antibiotic and } \\
\text { antimycotic reagents }\end{array}$ \\
\hline Alpha-MEM & 1000 & $\begin{array}{l}10.08 \mathrm{~g} \text { alpha-MEM } \\
\text { powder, } 2.2 \mathrm{~g} \text { sodium } \\
\text { bicarbonate }\end{array}$ & $\begin{array}{l}10 \mathrm{~mL} \text { Fungizone }{ }^{\circledR} \\
\text { amphotericin } \mathrm{B}, 5 \mathrm{~mL} \\
\text { gentamicin reagent } \\
\text { solution, } 10 \mathrm{~mL} \\
\text { penicillin } \mathrm{G} \text { potassium } \\
\text { salt, } 14 \mathrm{~mL} \text { HEPES } \\
\text { buffer solution }\end{array}$ \\
\hline 2X alpha-MEM & 500 & $\begin{array}{l}10.08 \mathrm{~g} \text { alpha-MEM } \\
\text { powder, } 2.2 \mathrm{~g} \text { sodium } \\
\text { bicarbonate }\end{array}$ & $\begin{array}{l}10 \mathrm{~mL} \text { Fungizone }{ }^{\circledR} \\
\text { amphotericin } \mathrm{B}, 5 \mathrm{~mL} \\
\text { gentamicin reagent } \\
\text { solution, } 10 \mathrm{~mL} \\
\text { penicillin } \mathrm{G} \text { potassium } \\
\text { salt, } 14 \mathrm{~mL} \text { HEPES } \\
\text { buffer solution }\end{array}$ \\
\hline
\end{tabular}


Table A3. Preparation of soil load mixture.

\begin{tabular}{|l|l|l|}
\hline Ingredient & Concentration $^{\mathrm{a}}$ & Volume $^{\mathrm{b}}$ \\
\hline Tryptone & $0.05 \mathrm{~g} / \mathrm{mL}$ & $35 \mu \mathrm{L}$ \\
\hline Bovine mucin & $0.004 \mathrm{~g} / \mathrm{mL}$ & $100 \mu \mathrm{L}$ \\
\hline BSA & $0.05 \mathrm{~g} / \mathrm{mL}$ & $25 \mu \mathrm{L}$ \\
\hline
\end{tabular}

${ }^{\mathrm{a} C}$ Concentration prepared in PBS.

${ }^{b}$ Volume of soil load mixture to be added to $340 \mu \mathrm{L}$ of virus inoculum. 


\section{Appendix B. Statistical Analysis of Virus Infectivity Control Tests and Viral Drying}

Table B1. Effect of soil load, neutralizer and disinfectant on the virus infectivity of MNV-1.

\begin{tabular}{|c|c|c|c|c|c|}
\hline Test* & $\begin{array}{l}\text { Disinfectant or } \\
\text { solution }\end{array}$ & $\begin{array}{l}\text { Mean PFU } \\
\text { (test solution) }\end{array}$ & $\begin{array}{l}\text { Mean PFU } \\
\text { (control) }\end{array}$ & $\mathrm{p}$-value** & $\begin{array}{l}\text { Significant } \\
\text { difference*** }\end{array}$ \\
\hline $\begin{array}{l}\text { Soil load against } \\
\text { control }\end{array}$ & Soil load & 21.0 & 25.7 & 0.22 & $\mathrm{No}^{\mathrm{a}}$ \\
\hline \multirow{2}{*}{$\begin{array}{l}\text { Neutralizer against } \\
\text { control }\end{array}$} & Letheen broth & 27.3 & \multirow[t]{2}{*}{25.7} & \multirow[t]{2}{*}{0.80} & \multirow[t]{2}{*}{$\mathrm{No}^{\mathrm{a}}$} \\
\hline & $\begin{array}{l}\text { Sodium } \\
\text { thiosulphate }\end{array}$ & 28.0 & & & \\
\hline \multirow{6}{*}{$\begin{array}{l}\text { Disinfectant with } \\
\text { neutralizer, } \\
\text { disinfectant } \\
\text { without neutralizer } \\
\text { and control }\end{array}$} & $\begin{array}{l}\text { Bleach } \\
(5400 \text { ppm })\end{array}$ & $25.3^{\mathrm{b}}, 24.7^{\mathrm{c}}$ & 25.7 & 0.97 & $\mathrm{No}^{\mathrm{a}}$ \\
\hline & CaviCide & $25.7^{\mathrm{b}}, 20.7^{\mathrm{c}}$ & 25.7 & 0.33 & $\mathrm{No}^{\mathrm{a}}$ \\
\hline & Virox 5 & $26.3^{\mathrm{b}}, 23.7^{\mathrm{c}}$ & 25.7 & 0.77 & $\mathrm{No}^{\mathrm{a}}$ \\
\hline & $\mathrm{T}^{3} 6(20 \mu \mathrm{L})$ & $19.3^{\mathrm{b}}, 23.3^{\mathrm{c}}$ & 25.7 & 0.20 & $\mathrm{No}^{\mathrm{a}}$ \\
\hline & $\begin{array}{l}\text { Accel } \\
(35000 \mathrm{ppm})\end{array}$ & $\begin{array}{l}4.7^{\mathrm{b}} ; \mathrm{NA}, \mathrm{CTE} \\
\text { observed }^{\mathrm{c}}\end{array}$ & 22.3 & 0.00048 & Yes $^{\mathrm{a}}$ \\
\hline & $\begin{array}{l}\text { Accel } \\
(17500 \mathrm{ppm})\end{array}$ & $13.7^{\mathrm{b}}, 10.0^{\mathrm{c}}$ & 22.3 & 0.00041 & Yes $^{\mathrm{d}, \mathrm{e}}$ \\
\hline
\end{tabular}


Table B2. Effect of soil load, neutralizer and disinfectant on the virus infectivity of FCV.

\begin{tabular}{|c|c|c|c|c|c|}
\hline Test* & $\begin{array}{l}\text { Disinfectant or } \\
\text { solution }\end{array}$ & $\begin{array}{l}\text { Mean PFU } \\
\text { (test solution) }\end{array}$ & $\begin{array}{l}\text { Mean PFU } \\
\text { (control) }\end{array}$ & p-value** & $\begin{array}{l}\text { Significant } \\
\text { difference*** }\end{array}$ \\
\hline $\begin{array}{l}\text { Soil load against } \\
\text { control }\end{array}$ & Soil load & 21.7 & 19.7 & 0.52 & $\mathrm{No}^{\mathrm{a}}$ \\
\hline \multirow{2}{*}{$\begin{array}{l}\text { Neutralizer against } \\
\text { control }\end{array}$} & Letheen broth & 20.7 & \multirow[t]{2}{*}{19.7} & \multirow[t]{2}{*}{0.67} & \multirow[t]{2}{*}{$\mathrm{No}^{\mathrm{a}}$} \\
\hline & $\begin{array}{l}\text { Sodium } \\
\text { thiosulphate }\end{array}$ & 18.7 & & & \\
\hline \multirow{6}{*}{$\begin{array}{l}\text { Disinfectant with } \\
\text { neutralizer, } \\
\text { disinfectant } \\
\text { without neutralizer } \\
\text { and control }\end{array}$} & $\begin{array}{l}\text { Bleach } \\
(5400 \mathrm{ppm})\end{array}$ & $15.0^{\mathrm{b}}, 11.3^{\mathrm{c}}$ & 19.7 & 0.018 & Yes $^{f}$ \\
\hline & CaviCide & $26.7^{\mathrm{b}}, 18.7^{\mathrm{c}}$ & 19.7 & 0.20 & $\mathrm{No}^{\mathrm{a}}$ \\
\hline & Virox 5 & $31.0^{\mathrm{b}}, 11.7^{\mathrm{c}}$ & 19.7 & 0.0021 & Yes $^{\mathrm{d}, \mathrm{e}}$ \\
\hline & $\mathrm{T}^{3} 6(20 \mu \mathrm{L})$ & $29.7^{\mathrm{b}}, 17.3^{\mathrm{c}}$ & 19.7 & 0.021 & $\mathrm{Yes}^{\mathrm{e}}$ \\
\hline & $\mathrm{T}^{3} 6(50 \mu \mathrm{L})$ & $38.0^{\mathrm{b}}, 23.3^{\mathrm{c}}$ & 19.7 & 0.00071 & Yes $^{\mathrm{d}, \mathrm{e}}$ \\
\hline & $\begin{array}{l}\text { Accel } \\
(35000 \mathrm{ppm})\end{array}$ & $\begin{array}{l}25.0^{\mathrm{b}} ; \mathrm{NA}, \\
\text { CTE observed }^{\mathrm{c}}\end{array}$ & 19.7 & 0.35 & $\mathrm{No}^{\mathrm{a}}$ \\
\hline
\end{tabular}

*All tests were done in triplicate $(\mathrm{n}=3)$. **ANOVA single factor. ***Tukey's Test. No significant difference at $\mathrm{p}>0.05$. ${ }^{\mathrm{a}}$ Tukey's Test not used. ${ }^{\mathrm{b}}$ Refers to disinfectant with neutralizer. ${ }^{\mathrm{c}}$ Refers to disinfectant without neutralizer. ${ }^{\mathrm{d}}$ Disinfectant with neutralizer and control were significantly different. ${ }^{\mathrm{e}}$ Disinfectant without neutralizer and disinfectant with neutralizer were significantly different.

${ }^{\mathrm{f}}$ Disinfectant without neutralizer and control were significantly different. NA refers to not applicable. CTE refers to cytotoxicity effects. The control consists of EBSS. 
Table B3. Effect of soil load on the virus infectivity of MNV-1.

ANOVA: Single Factor

SUMMARY

\begin{tabular}{lrrrr}
\hline \multicolumn{1}{c}{ Groups } & Count & \multicolumn{1}{c}{ Sum } & Average & Variance \\
\hline Control (EBSS) & 3 & 77 & 25.7 & 30.3 \\
EBSS + Soil Load & 3 & 63 & 21 & 1 \\
\hline
\end{tabular}

ANOVA

\begin{tabular}{lcccccc}
\hline Source of Variation & \multicolumn{1}{l}{$S S$} & $d f$ & $M S$ & $F$ & P-value & F crit \\
\hline Between Groups & 32.7 & 1 & 32.7 & 2.09 & 0.22 & 7.71 \\
Within Groups & 62.7 & 4 & 15.7 & & & \\
Total & 95.3 & 5 & & & & \\
\hline
\end{tabular}

Table B4. Effect of soil load on the virus infectivity of FCV.

ANOVA: Single Factor

SUMMARY

\begin{tabular}{lrrrr}
\hline \multicolumn{1}{c}{ Groups } & \multicolumn{1}{c}{ Count } & \multicolumn{1}{c}{ Sum } & Average & Variance \\
\hline Control (EBSS) & 3 & 59 & 19.7 & 10.3 \\
EBSS + Soil Load & 3 & 65 & 21.7 & 14.3 \\
\hline
\end{tabular}

ANOVA

\begin{tabular}{lrrrrrr}
\hline Source of Variation & \multicolumn{1}{c}{$S S$} & $d f$ & $M S$ & $F$ & P-value & F crit \\
\hline Between Groups & 6 & 1 & 6 & 0.49 & 0.52 & 7.71 \\
Within Groups & 49.3 & 4 & 12.3 & & & \\
Total & 55.3 & 5 & & & & \\
\hline
\end{tabular}


Table B5. Effect of sodium thiosulphate and letheen broth neutralizer on the virus infectivity of MNV-1.

ANOVA: Single Factor

SUMMARY

\begin{tabular}{lrrrr}
\hline \multicolumn{1}{c}{ Groups } & \multicolumn{1}{c}{ Count } & Sum & Average & Variance \\
\hline Letheen Broth & 3 & 82 & 27.3 & 16.3 \\
Sodium Thiosulphate & 3 & 84 & 28 & 9 \\
Control (EBSS) & 3 & 77 & 25.7 & 30.3 \\
\hline
\end{tabular}

ANOVA

\begin{tabular}{lrrrrrr}
\hline \multicolumn{1}{c}{ Source of Variation } & \multicolumn{1}{c}{$S S$} & $d f$ & \multicolumn{1}{c}{$M S$} & \multicolumn{1}{c}{$F$} & P-value & F crit \\
\hline Between Groups & 8.7 & 2 & 4.3 & 0.23 & 0.80 & 5.14 \\
Within Groups & 111.3 & 6 & 18.6 & & & \\
Total & 120 & 8 & & & & \\
\hline
\end{tabular}

Table B6. Effect of sodium thiosulphate and letheen broth neutralizer on the virus infectivity of FCV.

ANOVA: Single Factor

SUMMARY

\begin{tabular}{lrrrr}
\multicolumn{1}{c}{ Groups } & Count & Sum & Average & Variance \\
\hline Letheen Broth & 3 & 62 & 20.7 & 6.3 \\
Sodium Thiosulphate & 3 & 56 & 18.7 & 4.3 \\
Control (EBSS) & 3 & 59 & 19.7 & 10.3 \\
\hline
\end{tabular}

ANOVA

\begin{tabular}{lrrrrrr}
\hline \multicolumn{1}{c}{ Source of Variation } & \multicolumn{1}{c}{$S S$} & $d f$ & $M S$ & $F$ & P-value & F crit \\
\hline Between Groups & 6 & 2 & 3 & 0.43 & 0.67 & 5.14 \\
Within Groups & 42 & 6 & 7 & & & \\
Total & 48 & 8 & & & & \\
\hline
\end{tabular}


Table B7. Effect of disinfectant with neutralizer and disinfectant without neutralizer on the virus infectivity of MNV-1.

A

Bleach - 5400 ppm

ANOVA: Single Factor

SUMMARY

\begin{tabular}{lrrrr}
\hline \multicolumn{1}{c}{ Groups } & Count & Sum & Average & Variance \\
\hline No Neutralizer & 3 & 74 & 24.7 & 26.3 \\
Neutralizer & 3 & 76 & 25.3 & 21.3 \\
Control (EBSS) & 3 & 77 & 25.7 & 30.3 \\
\hline
\end{tabular}

ANOVA

\begin{tabular}{lrrrrrr}
\hline Source of Variation & \multicolumn{1}{c}{$S S$} & $d f$ & \multicolumn{1}{c}{$M S$} & $F$ & P-value & F crit \\
\hline Between Groups & 1.56 & 2 & 0.78 & 0.030 & 0.97 & 5.14 \\
Within Groups & 156 & 6 & 26 & & & \\
Total & 157.6 & 8 & & & & \\
\hline
\end{tabular}

B

Virox 5

ANOVA: Single Factor

SUMMARY

\begin{tabular}{lrrrr}
\multicolumn{1}{c}{ Groups } & Count & Sum & Average & Variance \\
\hline No Neutralizer & 3 & 71 & 23.7 & 26.3 \\
Neutralizer & 3 & 79 & 26.3 & 8.3 \\
Control (EBSS) & 3 & 77 & 25.7 & 30.3 \\
\hline
\end{tabular}

ANOVA

\begin{tabular}{lcrrrrr}
\hline Source of Variation & \multicolumn{1}{c}{$S S$} & $d f$ & $M S$ & $F$ & P-value & F crit \\
\hline Between Groups & 11.6 & 2 & 5.8 & 0.27 & 0.77 & 5.14 \\
Within Groups & 130 & 6 & 21.7 & & & \\
Total & 141.6 & 8 & & & & \\
\hline
\end{tabular}


C

\section{CaviCide}

ANOVA: Single Factor

SUMMARY

\begin{tabular}{lrrrr}
\hline \multicolumn{1}{c}{ Groups } & Count & \multicolumn{1}{c}{ Sum } & Average & Variance \\
\hline No Neutralizer & 3 & 62 & 20.7 & 4.3 \\
Neutralizer & 3 & 77 & 25.7 & 21.3 \\
Control (EBSS) & 3 & 77 & 25.7 & 30.3 \\
\hline
\end{tabular}

ANOVA

\begin{tabular}{lrrrrrr}
\hline Source of Variation & \multicolumn{1}{c}{$S S$} & $d f$ & \multicolumn{1}{c}{$M S$} & $F$ & P-value & F crit \\
\hline Between Groups & 50 & 2 & 25 & 1.34 & 0.33 & 5.14 \\
Within Groups & 112 & 6 & 18.7 & & & \\
Total & 162 & 8 & & & & \\
\hline
\end{tabular}

D

Accel - 35000 ppm

ANOVA: Single Factor

SUMMARY

\begin{tabular}{lrrrr}
\hline \multicolumn{1}{c}{ Groups } & \multicolumn{1}{c}{ Count } & Sum & Average & Variance \\
\hline Neutralizer & 3 & 14 & 4.7 & 4.3 \\
Control (EBSS) & 3 & 67 & 22.3 & 4.3 \\
\hline
\end{tabular}

ANOVA

\begin{tabular}{|c|c|c|c|c|c|c|}
\hline Source of Variation & $S S$ & $\overline{d f}$ & $M S$ & $F$ & $P$-value & $F$ crit \\
\hline Between Groups & 468.2 & 1 & 468.2 & 108.04 & 0.00048 & 7.71 \\
\hline Within Groups & 17.3 & 4 & 4.3 & & & \\
\hline Total & 485.5 & 5 & & & & \\
\hline
\end{tabular}


E

Accel - 17500 ppm

ANOVA: Single Factor

SUMMARY

\begin{tabular}{lrrrr}
\hline \multicolumn{1}{c}{ Groups } & Count & \multicolumn{1}{c}{ Sum } & Average & Variance \\
\hline No Neutralizer & 3 & 30 & 10 & 1 \\
Neutralizer & 3 & 41 & 13.7 & 4.3 \\
Control (EBSS) & 3 & 67 & 22.3 & 4.3 \\
\hline
\end{tabular}

ANOVA

\begin{tabular}{lrrrrrr}
\hline Source of Variation & \multicolumn{1}{c}{$S S$} & $d f$ & $M S$ & $F$ & P-value & F crit \\
\hline Between Groups & 240.7 & 2 & 120.3 & 37.34 & 0.00041 & 5.14 \\
Within Groups & 19.3 & 6 & 3.2 & & & \\
Total & 260 & 8 & & & & \\
\hline
\end{tabular}

F

$\mathrm{T}^{3} \mathbf{6}(20 \mu \mathrm{L})$

ANOVA: Single Factor

SUMMARY

\begin{tabular}{lrrrr}
\multicolumn{1}{c}{ Groups } & Count & \multicolumn{1}{c}{ Sum } & Average & Variance \\
\hline No Neutralizer & 3 & 70 & 23.3 & 12.3 \\
Neutralizer & 3 & 58 & 19.3 & 1.3 \\
Control (EBSS) & 3 & 77 & 25.7 & 30.3 \\
\hline
\end{tabular}

ANOVA

\begin{tabular}{lrrrrrr}
\hline Source of Variation & \multicolumn{1}{c}{$S S$} & $d f$ & \multicolumn{1}{c}{$M S$} & $F$ & P-value & \multicolumn{1}{c}{ F crit } \\
\hline Between Groups & 61.6 & 2 & 30.8 & 2.10 & 0.20 & 5.14 \\
Within Groups & 88 & 6 & 14.7 & & & \\
Total & 149.6 & 8 & & & & \\
\hline
\end{tabular}


Table B8. Effect of disinfectant with neutralizer and disinfectant without neutralizer on the virus infectivity of FCV.

A

Bleach - 5400 ppm

ANOVA: Single Factor

SUMMARY

\begin{tabular}{lrrrr}
\multicolumn{1}{c}{ Groups } & Count & \multicolumn{1}{c}{ Sum } & Average & Variance \\
\hline No Neutralizer & 3 & 34 & 11.3 & 4.3 \\
Neutralizer & 3 & 45 & 15 & 4 \\
Control (EBSS) & 3 & 59 & 19.7 & 10.3 \\
\hline
\end{tabular}

ANOVA

\begin{tabular}{lrrrrrr}
\hline Source of Variation & \multicolumn{1}{c}{$S S$} & $d f$ & $M S$ & $F$ & $P$-value & F crit \\
\hline Between Groups & 104.7 & 2 & 52.3 & 8.41 & 0.018 & 5.14 \\
Within Groups & 37.3 & 6 & 6.2 & & & \\
Total & 142 & 8 & & & & \\
\hline
\end{tabular}

B

CaviCide

ANOVA: Single Factor

SUMMARY

\begin{tabular}{lrrrr}
\multicolumn{1}{c}{ Groups } & Count & Sum & Average & Variance \\
\hline No Neutralizer & 3 & 56 & 18.7 & 26.3 \\
Neutralizer & 3 & 80 & 26.7 & 42.3 \\
Control (EBSS) & 3 & 59 & 19.7 & 10.3 \\
\hline
\end{tabular}

ANOVA

\begin{tabular}{llrrrrrr}
\hline Source of Variation & \multicolumn{1}{c}{$S S$} & $d f$ & $M S$ & $F$ & P-value & F crit \\
\hline Between Groups & 114 & 2 & 57 & 2.16 & 0.20 & 5.14 \\
Within Groups & 158 & & 6 & 26.3 & & & \\
& & & & & & & \\
Total & 272 & 8 & & & & \\
\hline
\end{tabular}


C

Virox 5

ANOVA: Single Factor

SUMMARY

\begin{tabular}{lrrrr}
\hline \multicolumn{1}{c}{ Groups } & Count & \multicolumn{1}{c}{ Sum } & Average & Variance \\
\hline No Neutralizer & 3 & 35 & 11.7 & 4.3 \\
Neutralizer & 3 & 93 & 31 & 27 \\
Control (EBSS) & 3 & 59 & 19.7 & 10.3 \\
\hline
\end{tabular}

ANOVA

\begin{tabular}{|c|c|c|c|c|c|c|}
\hline Source of Variation & $S S$ & $d f$ & $M S$ & $F$ & $P$-value & F crit \\
\hline Between Groups & 566.2 & 2 & 283.1 & 20.38 & 0.0021 & 5.14 \\
\hline Within Groups & 83.3 & 6 & 13.9 & & & \\
\hline Total & 649.6 & 8 & & & & \\
\hline
\end{tabular}

D

$\mathrm{T}^{3} \mathbf{6}(20 \mu \mathrm{L})$

ANOVA: Single Factor

SUMMARY

\begin{tabular}{lrrrr}
\multicolumn{1}{c}{ Groups } & Count & \multicolumn{1}{c}{ Sum } & Average & Variance \\
\hline No Neutralizer & 3 & 52 & 17.3 & 22.3 \\
Neutralizer & 3 & 89 & 29.7 & 16.3 \\
Control (EBSS) & 3 & 59 & 19.7 & 10.3 \\
\hline
\end{tabular}

ANOVA

\begin{tabular}{|c|c|c|c|c|c|c|}
\hline Source of Variation & $S S$ & $d f$ & $M S$ & $F$ & $P$-value & $F$ crit \\
\hline Between Groups & 257.6 & 2 & 128.8 & 7.88 & 0.021 & 5.14 \\
\hline Within Groups & 98 & 6 & 16.3 & & & \\
\hline Total & 355.6 & 8 & & & & \\
\hline
\end{tabular}


E

$\mathrm{T}^{3} 6(50 \mu \mathrm{L})$

ANOVA: Single Factor

SUMMARY

\begin{tabular}{lrrrr}
\hline \multicolumn{1}{c}{ Groups } & \multicolumn{1}{c}{ Count } & \multicolumn{1}{c}{ Sum } & Average & Variance \\
\hline No Neutralizer & 3 & 70 & 23.3 & 10.3 \\
Neutralizer & 3 & 114 & 38 & 7 \\
Control (EBSS) & 3 & 59 & 19.7 & 10.3 \\
\hline
\end{tabular}

ANOVA

\begin{tabular}{|c|c|c|c|c|c|c|}
\hline $\begin{array}{l}\text { Source of } \\
\text { Variation }\end{array}$ & $S S$ & $d f$ & $M S$ & $F$ & $P$-value & $F$ crit \\
\hline Between Groups & 564.7 & 2 & 282.3 & 30.61 & 0.00071 & 5.14 \\
\hline Within Groups & 55.3 & 6 & 9.2 & & & \\
\hline Total & 620 & 8 & & & & \\
\hline
\end{tabular}

F

Accel - 35000 ppm

ANOVA: Single Factor

SUMMARY

\begin{tabular}{lrrrr}
\hline \multicolumn{1}{c}{ Groups } & \multicolumn{1}{c}{ Count } & \multicolumn{1}{c}{ Sum } & Average & Variance \\
\hline Neutralizer & 3 & 75 & 25 & 67 \\
Control (EBSS) & 3 & 59 & 19.7 & 10.3 \\
\hline
\end{tabular}

ANOVA

\begin{tabular}{lrrrrrr}
\hline \multicolumn{1}{c}{$\begin{array}{l}\text { Source of } \\
\text { Variation }\end{array}$} & \multicolumn{1}{c}{ SS } & $d f$ & & & & \\
& & & $F$ & P-value & F crit \\
\hline Between Groups & 42.7 & 1 & 42.7 & 1.10 & 0.35 & 7.71 \\
Within Groups & 154.7 & 4 & 38.7 & & & \\
Total & 197.3 & 5 & & & & \\
\hline
\end{tabular}


Table B9. Determination of significant difference of the virus infectivity of MNV-1 using Tukey's HSD Test.

\begin{tabular}{|c|c|c|c|c|c|c|}
\hline Disinfectant & Treatment & HSD & $\begin{array}{l}\text { Mean } \\
\text { PFU }\end{array}$ & $\begin{array}{l}\text { Mean PFU } \\
\text { difference } \\
\text { from 1st row }\end{array}$ & \begin{tabular}{|l} 
Mean PFU \\
difference \\
from 2nd row
\end{tabular} & $\begin{array}{l}\text { Significant } \\
\text { difference }\end{array}$ \\
\hline $\begin{array}{l}\text { Bleach } \\
\text { (5400 ppm) }\end{array}$ & NA & NA & NA & NA & NA & No \\
\hline Virox 5 & NA & NA & NA & NA & NA & No \\
\hline CaviCide & NA & NA & NA & NA & NA & No \\
\hline $\begin{array}{l}\text { Accel } \\
(35000 \mathrm{ppm})\end{array}$ & NA & NA & NA & NA & NA & NA \\
\hline \multirow{3}{*}{$\begin{array}{l}\text { Accel } \\
(17500 \mathrm{ppm})\end{array}$} & No neutralizer & \multirow[t]{3}{*}{4.5} & 10.0 & & & \multirow[t]{3}{*}{ Yes } \\
\hline & Neutralizer & & 13.7 & 3.7 & & \\
\hline & Control & & 22.3 & 12.3 & 8.7 & \\
\hline $\mathrm{T}^{3} 6(20 \mu \mathrm{L})$ & NA & NA & NA & NA & NA & NA \\
\hline
\end{tabular}

Bolded values indicate significant difference. HSD refers to Honestly Significant Difference. NA refers to not applicable. 
Table B10. Determination of significant difference of the virus infectivity of FCV using Tukey's HSD Test.

\begin{tabular}{|c|c|c|c|c|c|c|}
\hline Disinfectant & Treatment & HSD & $\begin{array}{l}\text { Mean } \\
\text { PFU }\end{array}$ & $\begin{array}{l}\text { Mean PFU } \\
\text { difference } \\
\text { from 1st row }\end{array}$ & $\begin{array}{l}\text { Mean PFU } \\
\text { difference } \\
\text { from 2nd row }\end{array}$ & $\begin{array}{l}\text { Significant } \\
\text { difference }\end{array}$ \\
\hline \multirow{3}{*}{$\begin{array}{l}\text { Bleach } \\
(5400 \text { ppm) }\end{array}$} & No neutralizer & \multirow[t]{3}{*}{6.3} & 11.3 & & & \multirow[t]{3}{*}{ Yes } \\
\hline & Neutralizer & & 15.0 & 3.7 & & \\
\hline & Control & & 19.7 & 8.3 & 4.7 & \\
\hline \multirow[t]{3}{*}{ Virox 5} & No neutralizer & \multirow[t]{3}{*}{9.3} & 11.7 & & & \multirow[t]{3}{*}{ Yes } \\
\hline & Neutralizer & & 31.0 & 19.3 & & \\
\hline & Control & & 19.7 & 8.0 & 11.3 & \\
\hline \multirow[t]{3}{*}{ CaviCide } & No neutralizer & \multirow[t]{3}{*}{12.9} & 18.7 & & & \multirow[t]{3}{*}{ No } \\
\hline & Neutralizer & & 26.7 & 8.0 & & \\
\hline & Control & & 19.7 & 1.0 & 7.0 & \\
\hline $\begin{array}{l}\text { Accel } \\
(35000 \mathrm{ppm})\end{array}$ & NA & NA & NA & NA & NA & NA \\
\hline $\begin{array}{l}\text { Accel } \\
(17500 \mathrm{ppm})\end{array}$ & NT & NT & NT & NT & NT & NT \\
\hline \multirow[t]{3}{*}{$\mathrm{T}^{3} 6(20 \mu \mathrm{L})$} & No neutralizer & \multirow[t]{3}{*}{10.1} & 17.3 & & & \multirow[t]{3}{*}{ Yes } \\
\hline & Neutralizer & & 29.7 & 12.3 & & \\
\hline & Control & & 19.7 & 2.3 & 10.0 & \\
\hline \multirow[t]{3}{*}{$\mathrm{T}^{3} 6(50 \mu \mathrm{L})$} & No neutralizer & \multirow[t]{3}{*}{7.6} & 23.3 & & & \multirow[t]{3}{*}{ Yes } \\
\hline & Neutralizer & & 38.0 & 14.7 & & \\
\hline & Control & & 19.7 & 3.7 & 18.3 & \\
\hline
\end{tabular}

Bolded values indicate significant difference. HSD refers to Honestly Significant Difference. NA refers to not applicable. NT refers to not tested. 
Table B11. Comparison of viral titer remaining (\%) of MNV-1 and FCV without soil load.

t-Test: Two-Sample Assuming Unequal Variances

\begin{tabular}{lrr}
\hline & MNV-1 without Soil Load & FCV without Soil Load \\
\hline Mean & 0.20 & 0.43 \\
Variance & 0.0042 & 0.025 \\
Observations & 11 & 11 \\
Hypothesized Mean Difference & 0 & \\
df & 13 & \\
t Stat & -4.52 & \\
P $(\mathrm{T}<=t)$ two-tail & 0.00058 & \\
t Critical two-tail & 2.16 & \\
\hline
\end{tabular}

Table B12. Comparison of viral titer remaining (\%) of MNV-1 and FCV with soil load.

t-Test: Two-Sample Assuming Unequal Variances

\begin{tabular}{lrr}
\hline & $M N V-1$ with Soil Load & FCV with Soil Load \\
\hline Mean & 0.18 & 0.38 \\
Variance & 0.0033 & 0.016 \\
Observations & 5 & 7 \\
Hypothesized Mean Difference & 0 & \\
df & 9 & \\
t Stat & -3.78 & \\
P $($ T<=t) two-tail & 0.0043 & \\
t Critical two-tail & 2.26 & \\
\hline
\end{tabular}


Table B13. Comparison of viral titer remaining (\%) of MNV-1 with and without soil load.

t-Test: Two-Sample Assuming Unequal Variances

\begin{tabular}{lrr}
\hline & MNV-1 without Soil Load & MNV-1 with Soil Load \\
\hline Mean & 0.20 & 0.18 \\
Variance & 0.0042 & 0.0033 \\
Observations & 11 & 5 \\
Hypothesized Mean Difference & 0 & \\
df & 9 & \\
t Stat & 0.76 & \\
P(T<=t) two-tail & 0.47 & \\
t Critical two-tail & 2.26 & \\
\hline
\end{tabular}

Table B14. Comparison of viral titer remaining (\%) of FCV with and without soil load.

t-Test: Two-Sample Assuming Unequal Variances

\begin{tabular}{lrr}
\hline & FCV without Soil Load & FCV with Soil Load \\
\hline Mean & 0.43 & 0.38 \\
Variance & 0.025 & 0.016 \\
Observations & 11 & 7 \\
Hypothesized Mean Difference & 0 & \\
df & 15 & \\
t Stat & 0.80 & \\
P(T<=t) two-tail & 0.44 & \\
t Critical two-tail & 2.13 & \\
\hline
\end{tabular}




\section{Appendix C. Photo Images of Experimental Design and Virus Plaques}

A

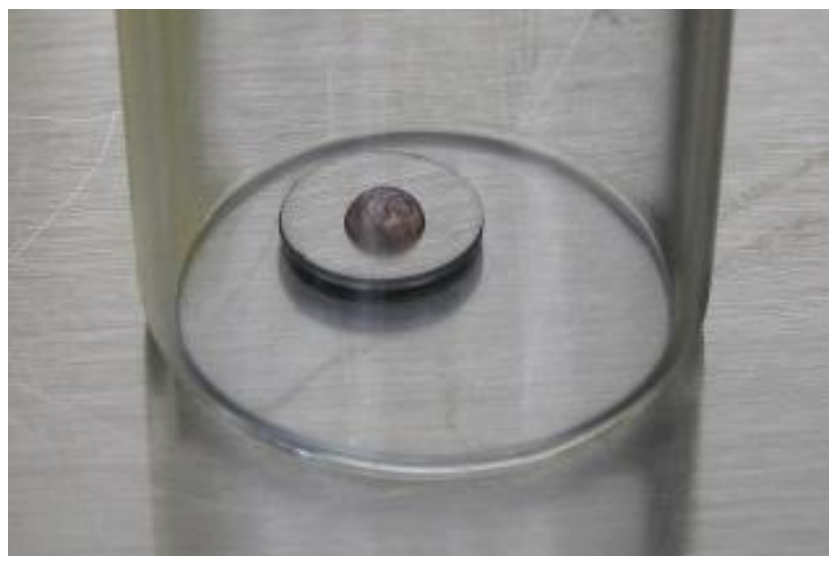

B

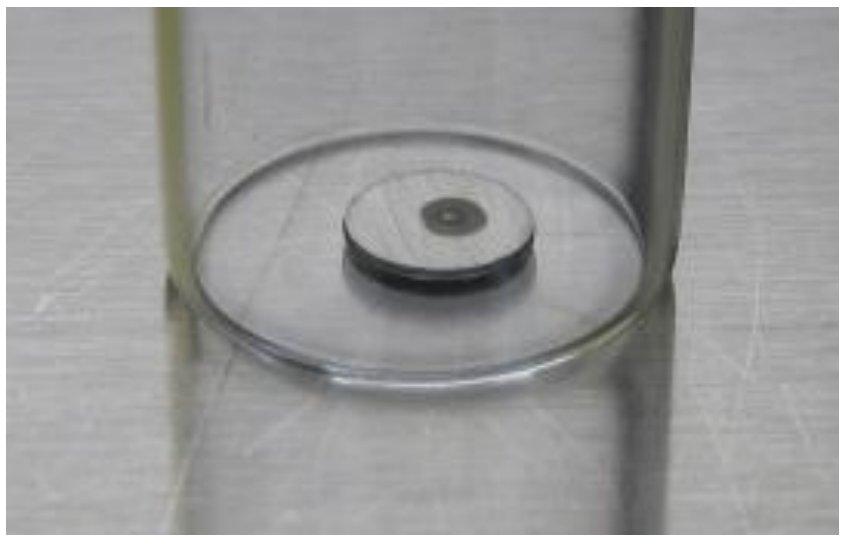

C

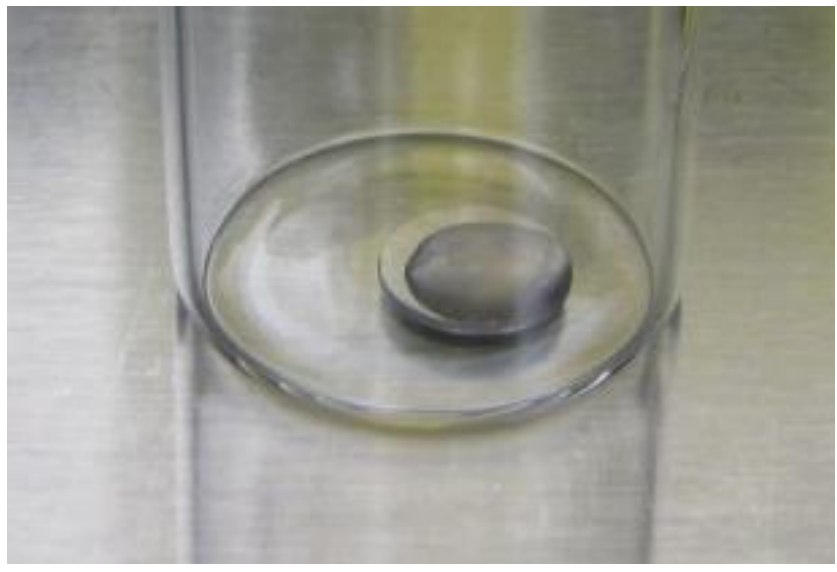

Figure C1. Stainless steel disc inoculated with $10 \mu \mathrm{L}$ of virus under (A) wet load condition, (B) dry load condition, and (C) with addition of $50 \mu \mathrm{L}$ of disinfectant. 
A

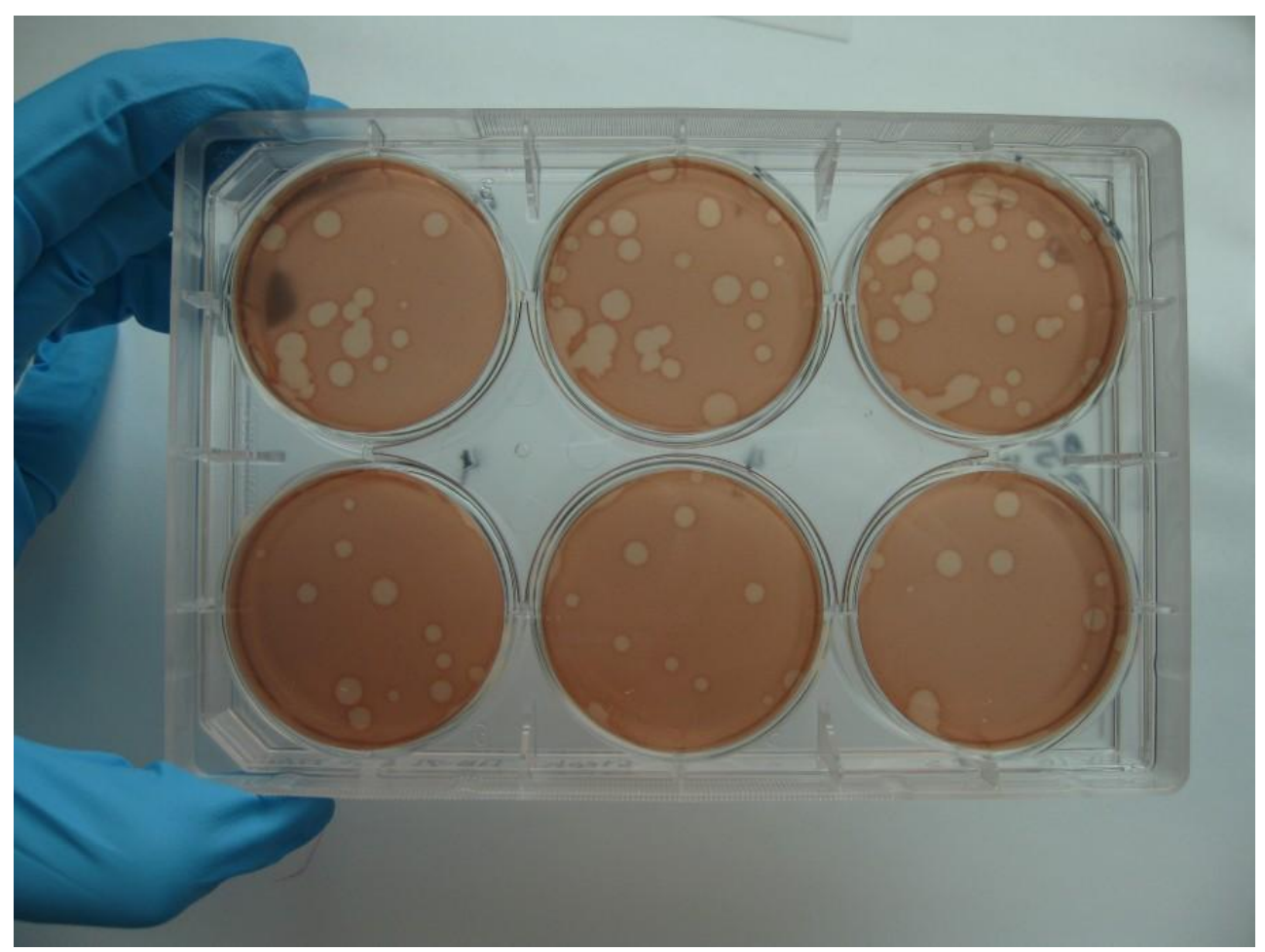

B

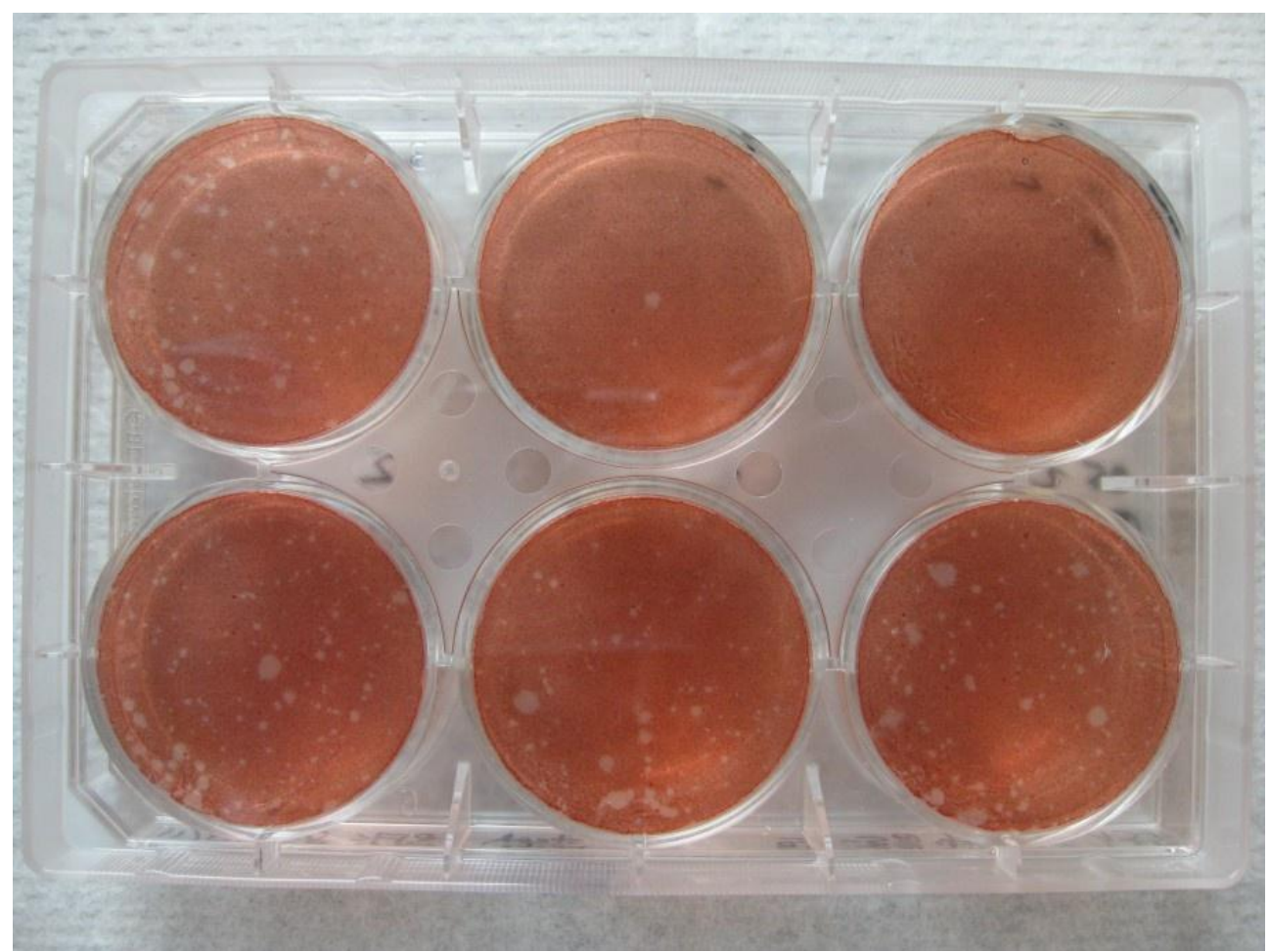

Figure C2. Virus plaques visible on 6-well plates, with (A) MNV-1 virus plaques and (B) FCV virus plaques. 
A

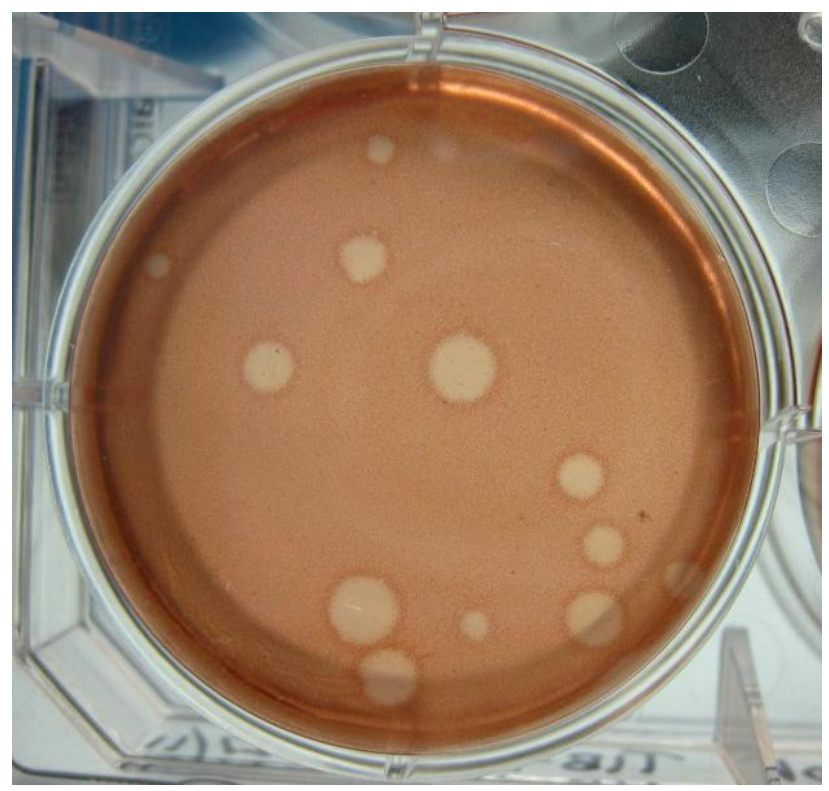

B

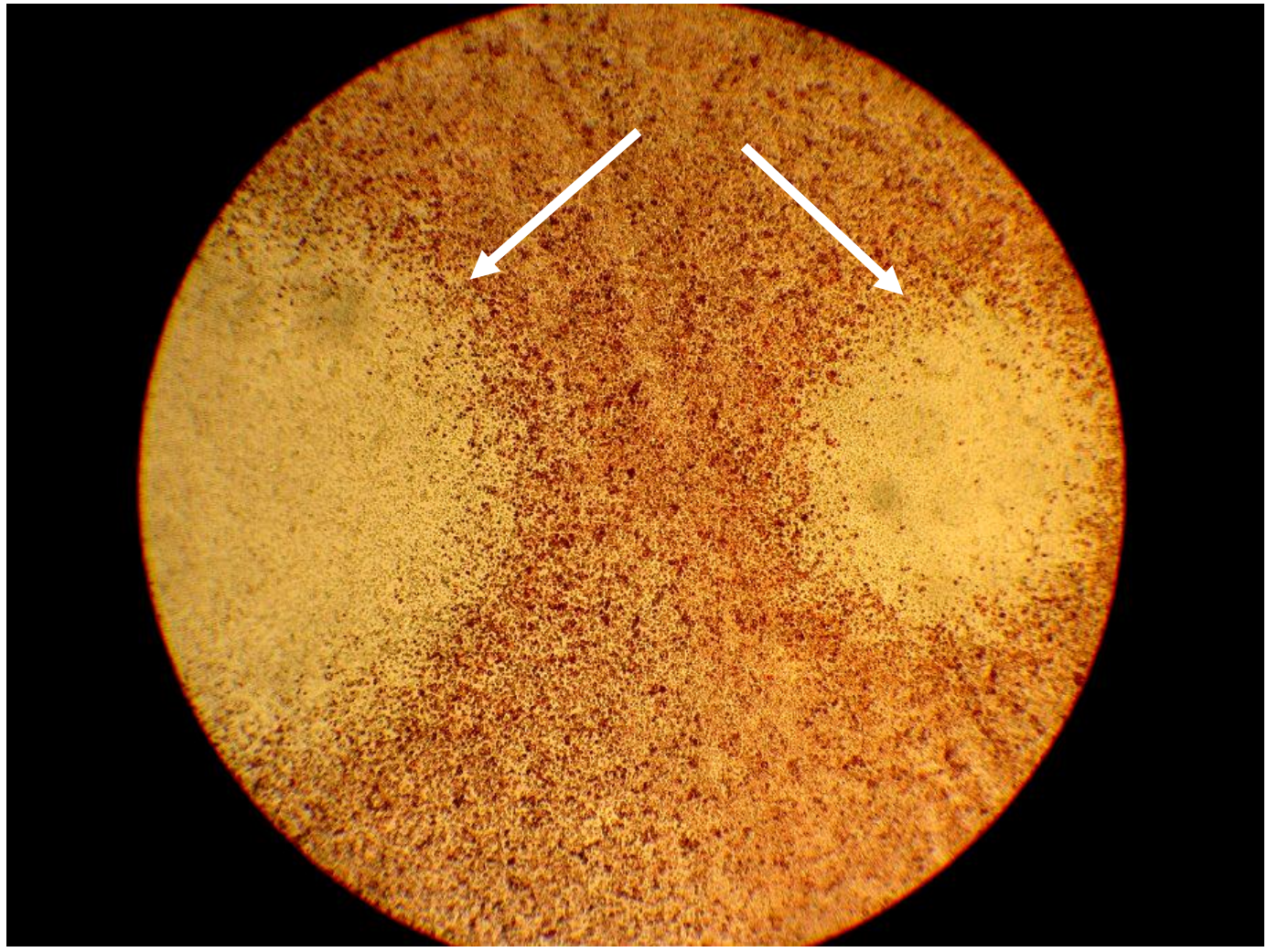

Figure $\mathrm{C} 3$. MNV-1 virus plaque under (A) naked eye and (B) microscope at 40x magnification. 
A

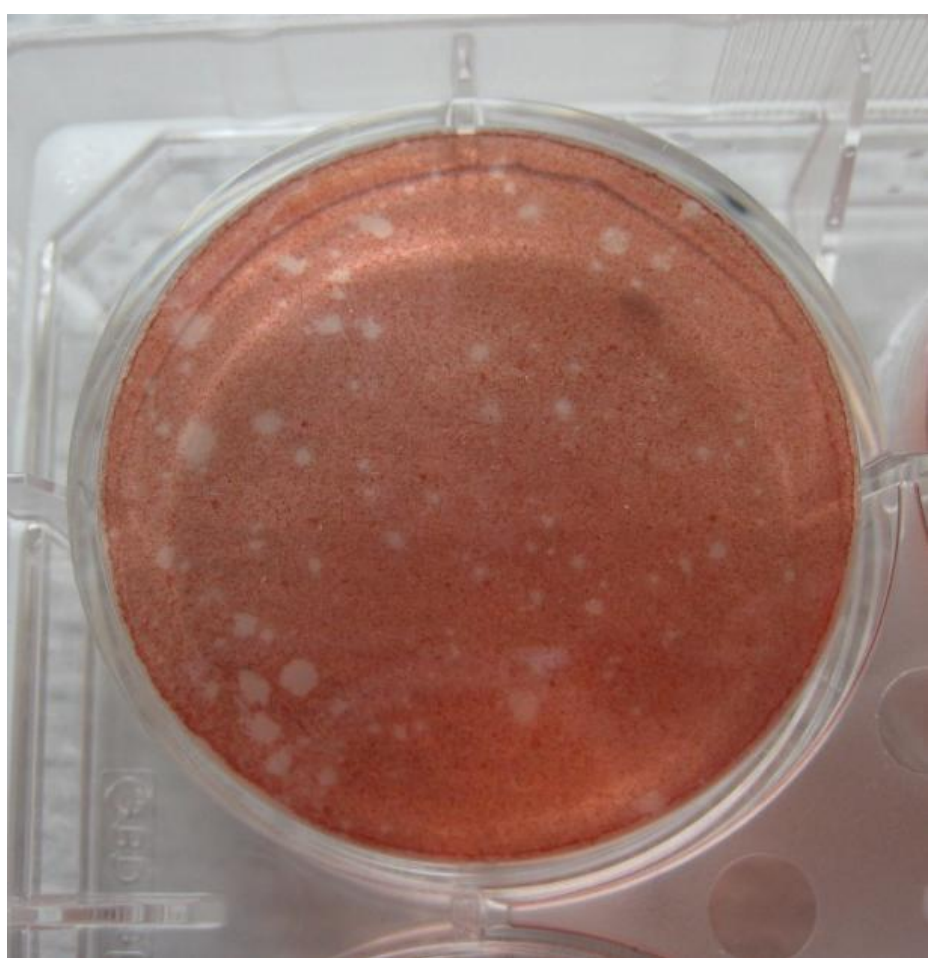

B

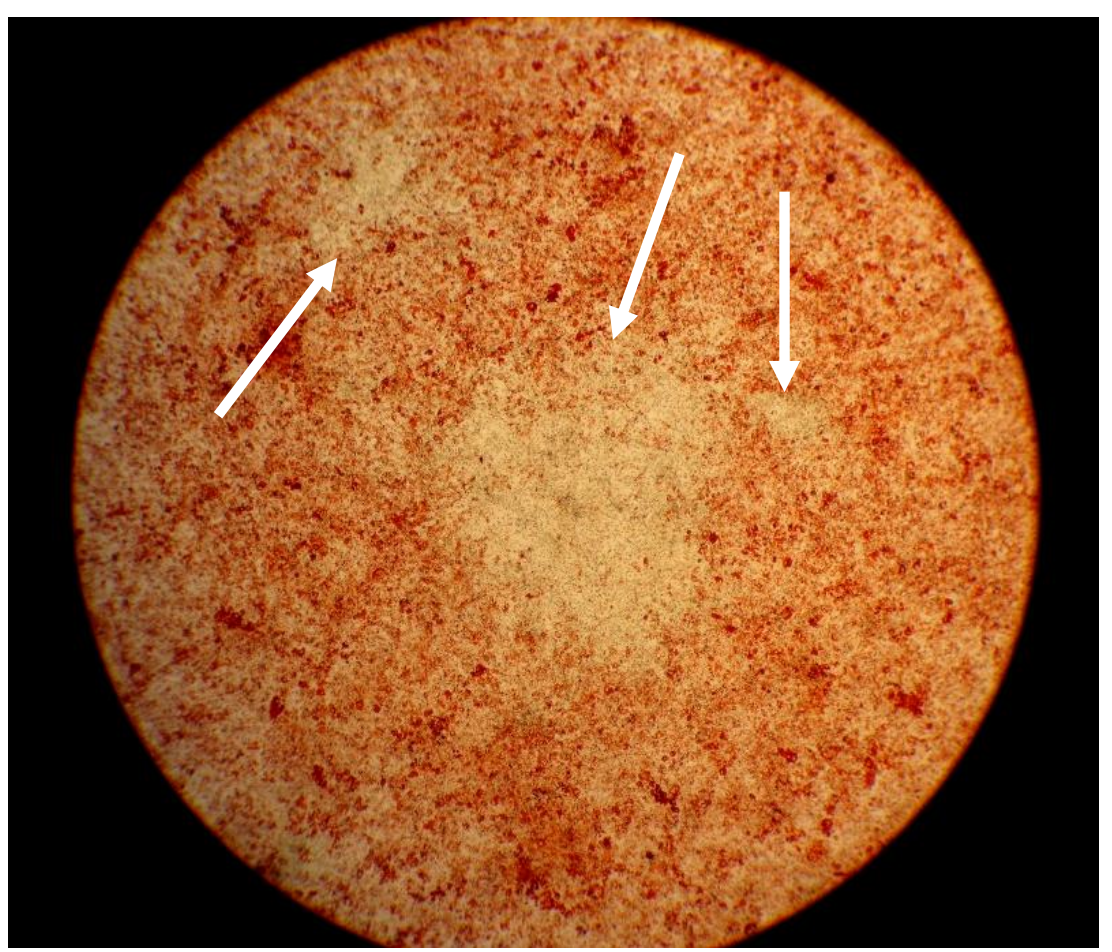

Figure C4. FCV virus plaque under (A) naked eye and (B) microscope at 40x magnification. 
A
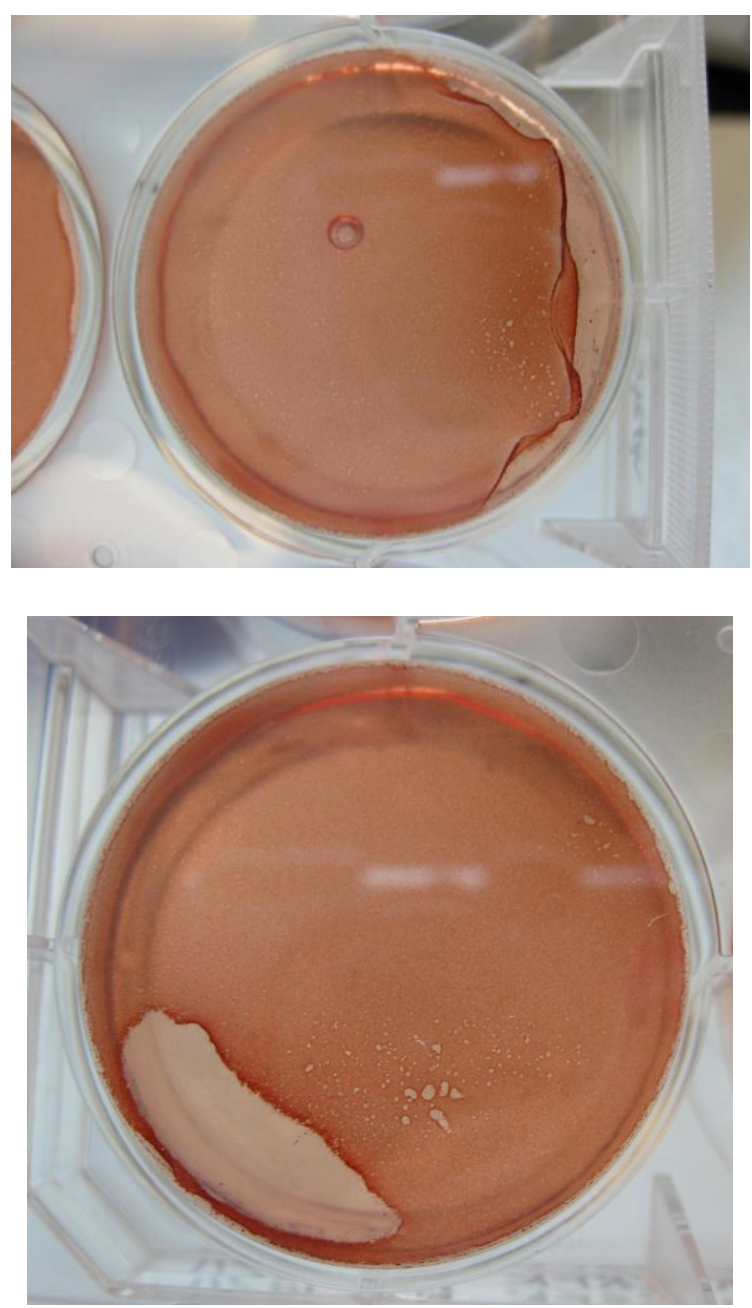

B

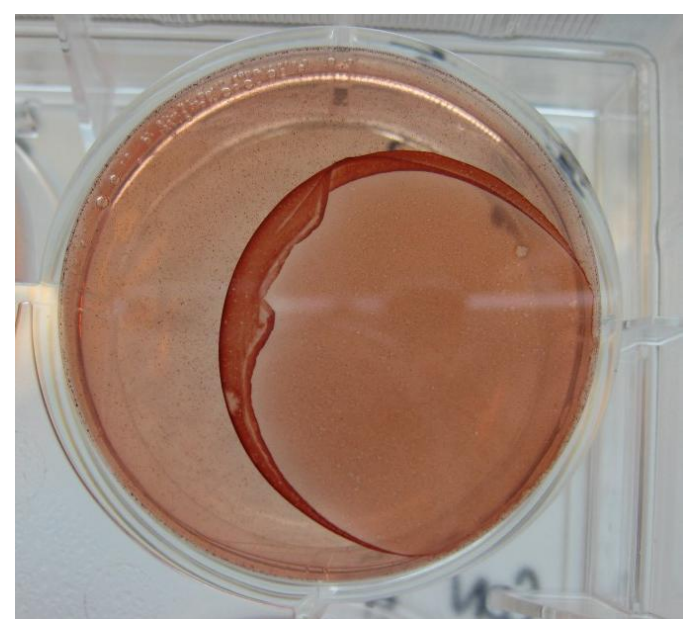

Figure C5. Monolayer detachment of (A) RAW 264.7 cells and (B) CRFK cells, on 6-well plate due to improper and quick application of agarose overlay on to well plate. 


\section{Appendix D. Conversion of Revolutions Per Minute (rpm) to Centrifugal Force $(\times g)$}

\section{Equation:}

$$
\text { Centrifugal force }(\times g)=1.118 \times 10^{-5} \times \mathrm{R} \times \mathrm{N}^{2}
$$

$\mathrm{R}=$ maximum effective rotating radius of centrifuge head measured in $\mathrm{cm}$ from centre of drive shaft to far end of contents of centrifuge tube in its extended position $\mathrm{N}=$ revolutions per minute $(\mathrm{rpm})$

Source: Grist et al., 1979.

\section{Calculations of conversions:}

Table D1. Conversion of Revolutions Per Minute $(\mathrm{rpm})$ to Centrifugal Force $(\times g)$

\begin{tabular}{|l|l|l|l|}
\hline Equipment & $\begin{array}{l}\text { Revolutions } \\
\text { per minute } \\
(\mathrm{rpm})\end{array}$ & $\begin{array}{l}\text { Maximum rotating } \\
\text { radius of centrifuge } \\
\text { head }(\mathrm{cm})\end{array}$ & Centrifugal force $(\times g)$ \\
\hline $\begin{array}{l}\text { Heraeus Clinifuge 75003539 } \\
\text { rotor Sepatech 3760 }\end{array}$ & 2000 & 9.65 & 432 \\
\hline $\begin{array}{l}\text { Hettich Zentrifugen Rotanta } \\
\text { 460 centrifuge }\end{array}$ & 3000 & 18.8 & 1892 \\
\hline
\end{tabular}

